\title{
Predicting galling behaviour in deep drawing processes
}

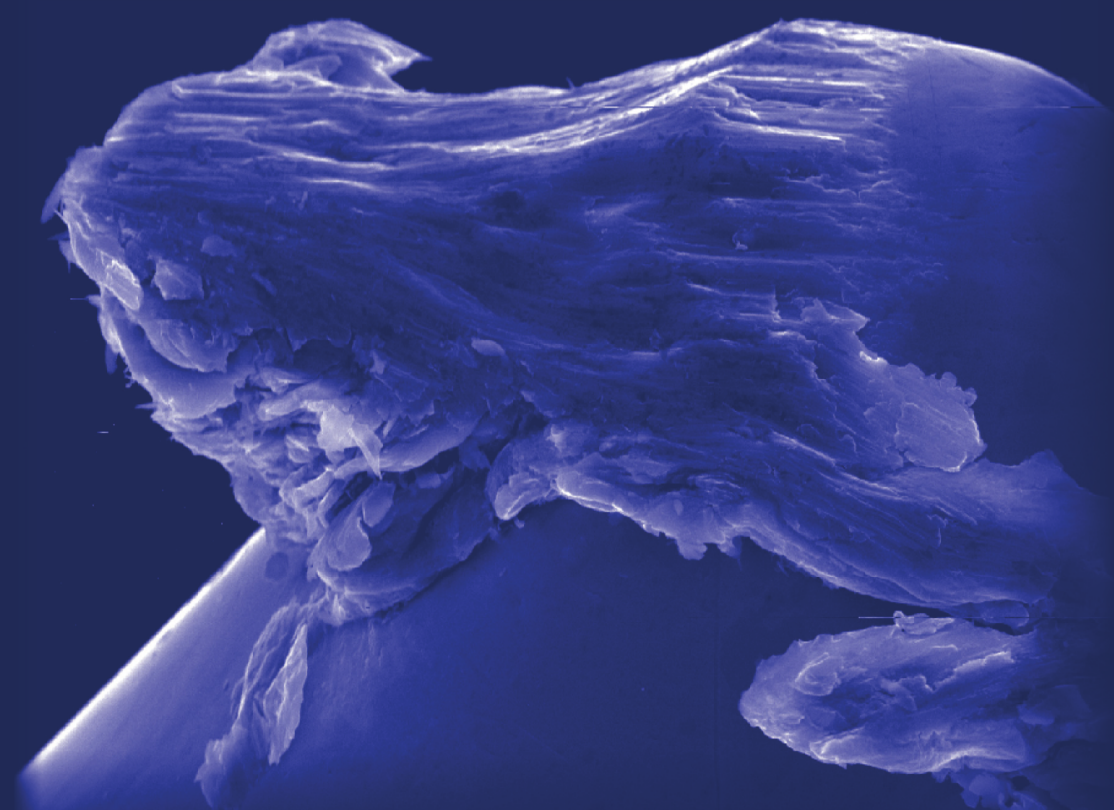

Gerrit van der Linde 


\section{Predicting galling behaviour in deep drawing processes}

Gerrit van der Linde 
This research was carried out under the project number MC1.03160 in the framework of the Research Program of the Materials innovation institute M2i (www.m2i.nl), the former Netherlands Institute for Metals Research.

De promotiecommissie is als volgt samengesteld:

$\begin{array}{lll}\text { prof.dr. F. Eising } & \text { Universiteit Twente } & \text { voorzitter en secretaris } \\ \text { prof.dr.ir. D.J. Schipper } & \text { Universiteit Twente } & \text { promotor } \\ \text { dr.ir. M.B. de Rooij } & \text { Universiteit Twente } & \text { assistent promotor } \\ \text { prof.dr.ir. R. Akkerman } & \text { Universiteit Twente } & \\ \text { prof.dr.ir. F.J.A.M. van Houten } & \text { Universiteit Twente } \\ \text { prof.dr.ir. J. Huetink } & \text { Universiteit Twente } \\ \text { prof.dr.ir. P. de Baets } & \text { Universiteit Gent, België } \\ \text { prof.dr.ir. L. Katgerman } & \text { Technische Universiteit Delft }\end{array}$

Van der Linde, Gerrit

Predicting galling behaviour in deep drawing processes

$\mathrm{PhD}$ Thesis, University of Twente, Enschede, the Netherlands, November 2011

Keywords: tribology, sheet metal forming, deep drawing, material transfer, galling, galling model

ISBN: 978-90-365-3284-6

Copyright (C) 2011 by G. van der Linde, Enschede, the Netherlands Printed by Ipskamp Drukkers, Enschede, the Netherlands 
PREDICTING GALLING BEHAVIOUR IN DEEP DRAWING PROCESSES

\title{
PROEFSCHRIFT
}

\author{
ter verkrijging van \\ de graad van doctor aan de Universiteit Twente, \\ op gezag van de rector magnificus, \\ prof. dr. H. Brinksma \\ volgens besluit van het College voor Promoties \\ in het openbaar te verdedigen \\ op donderdag 17 november 2011 om 12.45 uur
}

door

Gerrit van der Linde

geboren op 13 augustus 1980

te Zwolle 
Dit proefschrift is goedgekeurd door: de promotor: prof.dr.ir. D.J. Schipper de assistent promotor: dr.ir. M.B. de Rooij 


\section{Samenvatting}

Dieptrekken is een plaatomvormproces dat veel wordt toegepast in bijvoorbeeld de automobielindustrie. Dit proces is vooral geschikt voor serieproduktie van plaatdelen met een complexe geometrie. De gereedschappen die voor dit proces nodig zijn moeten aan hoge eisen voldoen, omdat deze plaatdelen moeten vervaardigen met strikte kwaliteitseisen en tegelijkertijd grote krachten moeten kunnen weerstaan. Dientengevolge zijn de gereedschappen kostbaar. Oppervlaktebeschadigingen op het gereedschap kunnen tot uitval van de productie leiden. Eén van de faalmechanismen die dit kan veroorzaken is galling. Galling is een mechanisme waarbij materiaaloverdracht plaatsvindt van de plaat naar het gereedschap, waar 'klodders' gevormd worden op het oppervlak. Dit veroorzaakt vervolgens krassen in het plaatmateriaal. Tot op heden was het niet goed mogelijk om het optreden van galling in een industrieel plaatomvormproces te voorspellen. In dit proefschrift wordt een model gepresenteerd waarmee de het optreden van galling in een plaatomvormproces kan worden voorspeld.

Om materiaaloverdracht van een plaat- naar een gereedschapoppervlak te onderzoeken zijn experimenten uitgevoerd op de schaal van een enkele ruwheidstop. Resultaten van deze experimenten zijn gebruikt om een aangroeimodel te formuleren voor een enkele ruwheidstop. Naast adhesie tussen het overgedragen materiaal en het gereedschapoppervlak is er rekening gehouden met de mechanische stabiliteit van de gevormde klodder.

Om het optreden van galling in het contact tussen het plaatmateriaal en het gereedschap in een industrieel plaatomvormproces te analyseren, is er een aangroeimodel ontwikkeld voor meerdere ruwheidstoppen. Dit gallingmodel is gebaseerd op een bestaand contactmodel en het aangroeimodel voor een enkele ruwheidstop. Het ontwikkelde model is gebruikt om de invloed van een aantal parameters op galling in het dieptrekproces te onderzoeken. Volgens het model zijn de glijweg, de contactdruk en de relatieve sterkte van het overgedragen materiaal ten opzichte van het oorspronkelijke plaatmateriaal belangrijke factoren voor galling.

Op basis van de resultaten van het ontwikkelde model is een Galling Prestatie Indicator (GPI) geformuleerd. De indicator wordt bepaald door twee elementen op twee verschillende lengteschalen: Ten eerste door een factor die bepaald wordt op de schaal van meerdere contact makende ruwheidstoppen. Deze factor kan worden uitgerekend met het gallingmodel. Ten tweede wordt de Galling Prestatie Indicator bepaald door factoren die voortkomen uit de operationele condities van het dieptrekproces als geheel. Belangrijke grootheden in deze context zijn de contactdruk op een bepaalde locatie van het gereedschap 
en de glijweg die wordt afgelegd langs het oppervlak van het gereedschap op die locatie. Deze grootheden kunnen worden verkregen met behulp van een eindige elementen simulatie van het dieptrekproces. De indicator is zondanig geformuleerd dat deze kan worden geïmplementeerd als een post processor voor eindige elementen simulaties van het omvormproces. Het gallingmodel is gevalideerd met galling experimenten en succesvol toegepast op het dieptrekken van een cup. 


\section{Summary}

Deep drawing is a sheet metal forming process which is widely used in, for example, the automotive industry. With this process it is possible to form complex shaped parts of sheet metal and it is suitable for products that have to be produced in large numbers. The tools for this process are required to meet high demands, because these tools have to create high quality products while at the same time withstanding large forces. As a result, tooling is expensive. Damage might cause tools to fail during production and one such failure mechanism is galling. Galling is a mechanism whereby material transfer occurs from the sheet to the tool, where it forms lumps on the surface, and these lumps subsequently cause scratching into the sheet. Currently the occurrence of galling in real sheet metal forming applications is rather unpredictable. In this thesis a model is presented from which the galling tendency of a sheet material in forming operations can be predicted.

To investigate the phenomenon of material transfer from a sheet to a tool surface, experiments are performed on a single asperity scale. Observations from these experiments are used to formulate a single asperity lump growth model. Beside adhesion between the transferred material and the tool surface, the mechanical stability of the formed lump is taken into account.

In order to approach the galling situation in real contact between a sheet and a tool, a multi asperity lump growth model is developed. The multi asperity lump growth model is based on a developed contact model combined with the single asperity lump growth model. The multi asperity model is used to investigate the influence of a number of parameters on galling in deep drawing. According to the model, important parameters are the sliding distance, the contact pressure and the relative strength of the lump compared to the sheet material.

A galling performance indicator is formulated on the basis of results from the multi asperity lump growth model. The results are split into two components: a galling impact factor, which is determined on contact scale, and operational conditions, contact pressure and sliding length, that are obtained from a finite element simulation of the deep drawing process. The indicator is formulated in such a way that it can be implemented as a post processor for finite element simulations. The galling model is validated by galling tests and successfully applied to deep drawing of a cup. 


\section{Contents}

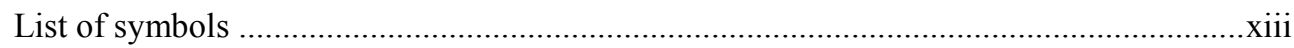

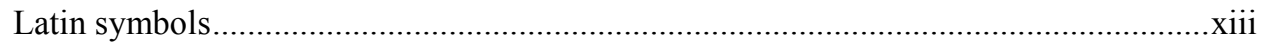

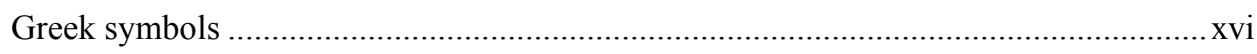

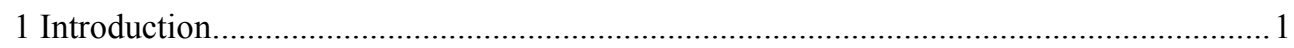

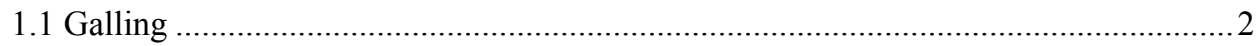

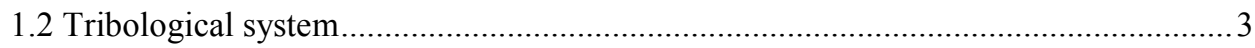

1.3 Objective of the research: development of a Galling Performance Indicator .............. 6

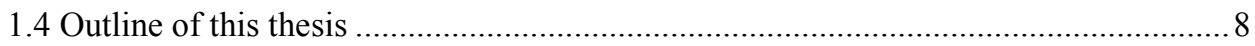

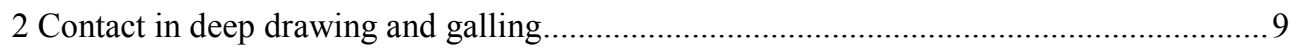

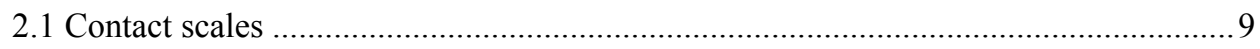

2.1.1 Macro scale

2.1.2 Meso scale

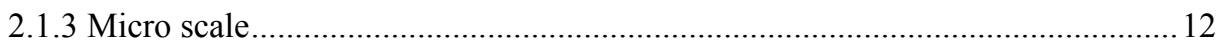

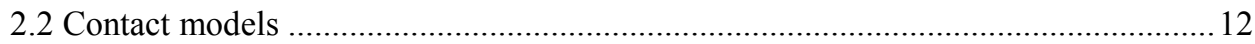

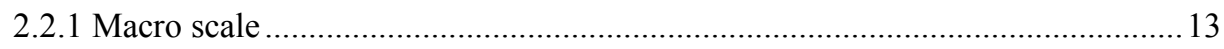

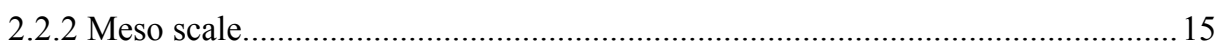

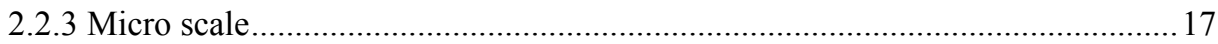

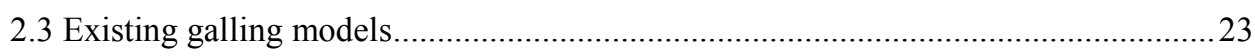

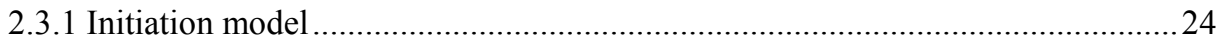

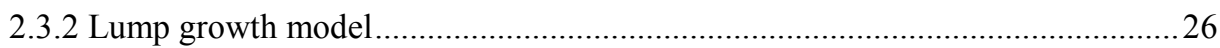

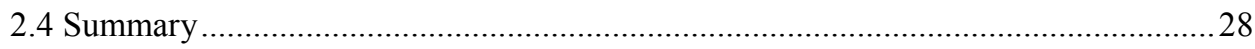

3 Material transfer on a single asperity scale: experiments.............................................29

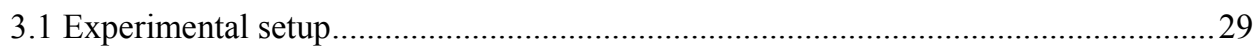

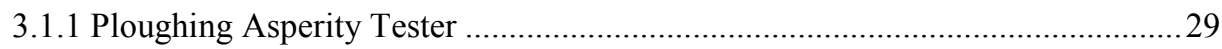

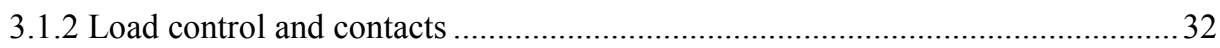

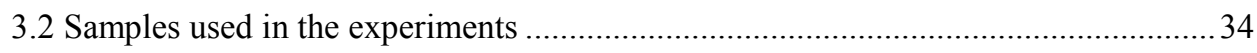

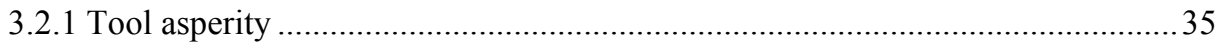

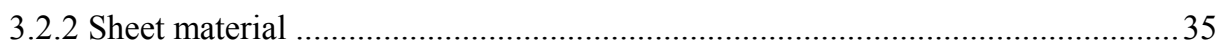

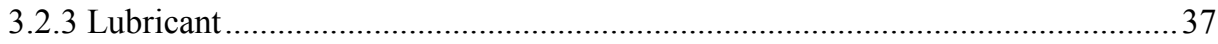

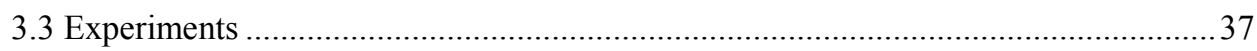

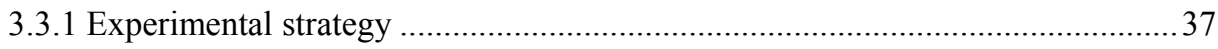

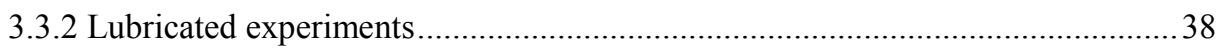

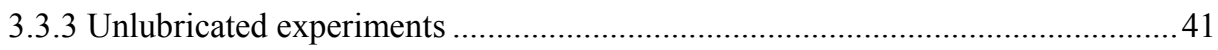

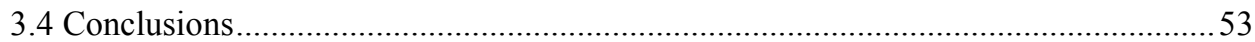

4 Material transfer and lump formation on a single asperity .............................................55

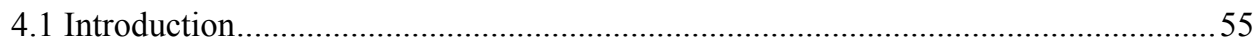




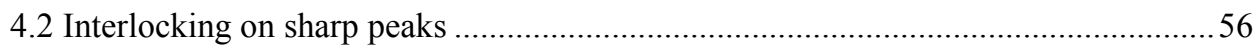

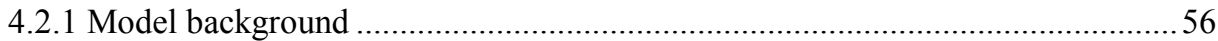

4.2.2 Model describing the formation of a dead metal zone ......................................58

4.3 Stability criterion for pyramidal shaped asperities...............................................6 63

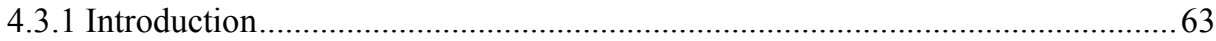

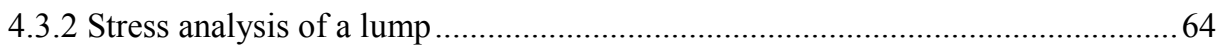

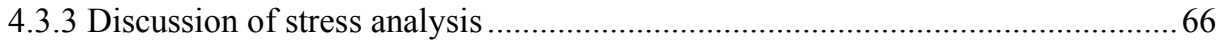

4.3.4 Model with an extended base of the asperity ............................................... 70

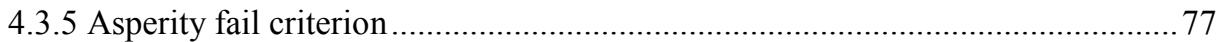

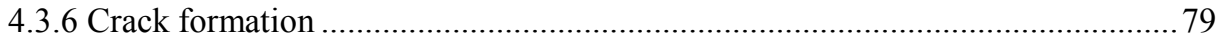

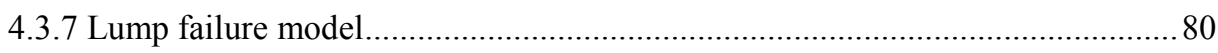

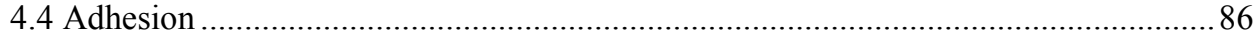

4.4.1 Geometry and material aspects of adhesion ................................................. 87

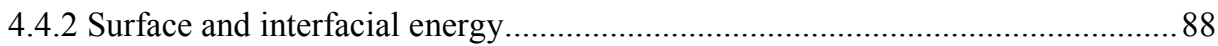

4.4.3 Surface and interfacial energy in practice ................................................... 90

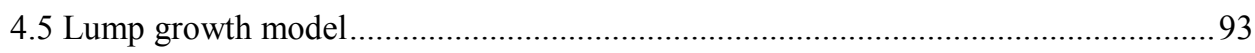

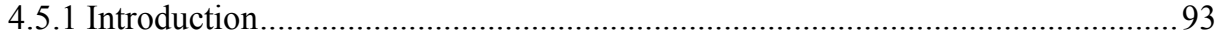

4.5.2 Volume of transferred material ................................................................... 93

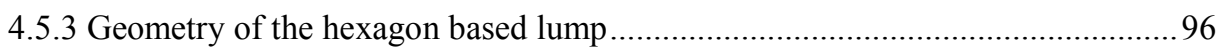

4.5.4 Deposition of transfer layer on hexagon based lump ....................................... 97

4.5.5 Stresses inside hexagon based lump ............................................................... 99

4.5.6 Stability and material redistribution of the asperity ........................................ 105

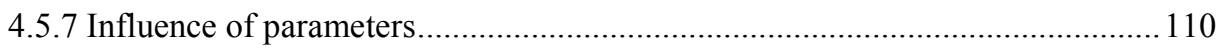

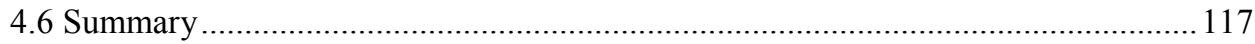

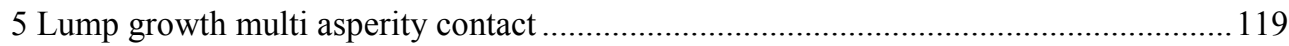

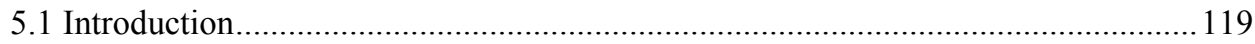

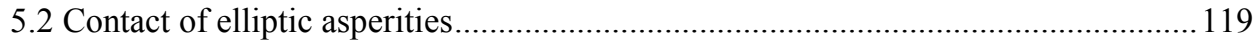

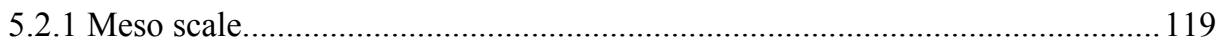

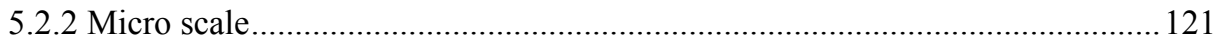

5.3 Conversion from ellipse to hexagon based asperity ......................................... 125

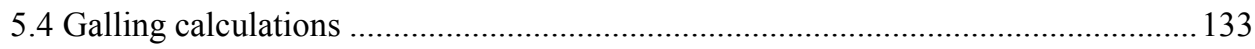

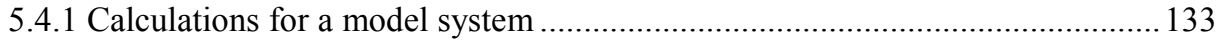

5.4.2 Consequences for galling in deep drawing processes ...................................... 145

5.5 Comparison between model and practice ............................................................. 146

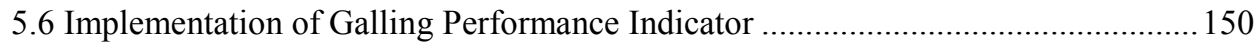

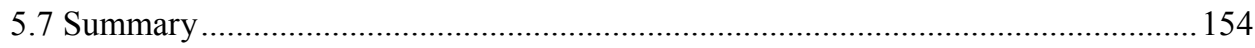

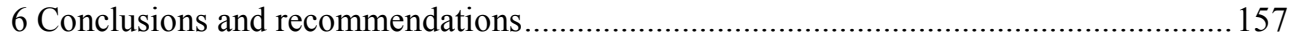

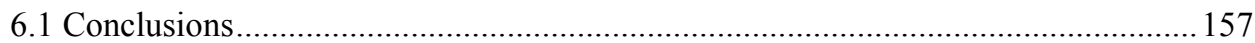

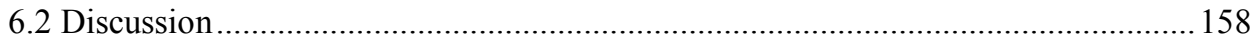




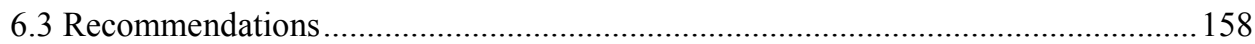

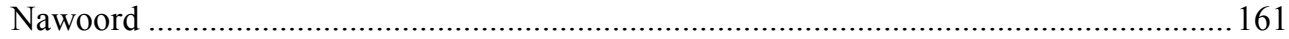

Appendix A: Stresses in pyramidal and hexagon based asperities .................................. 163

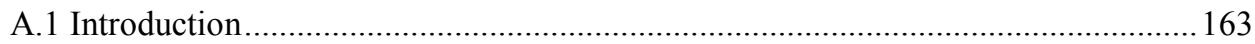

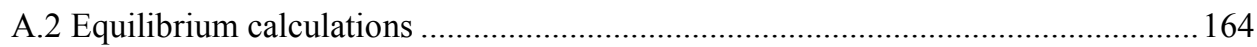

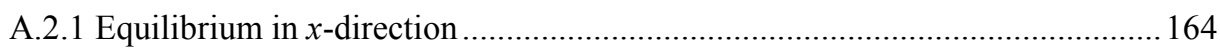

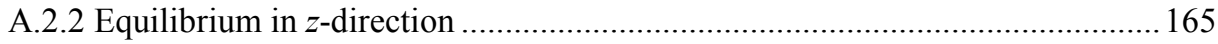

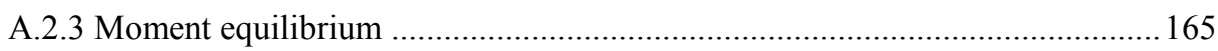

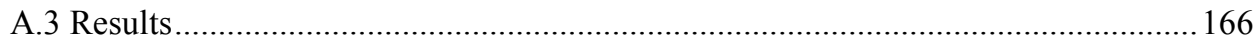

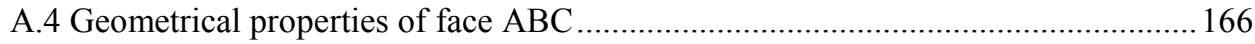

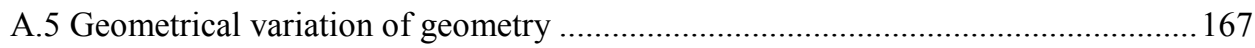

Appendix B: Geometry and material aspects of adhesion ............................................. 171

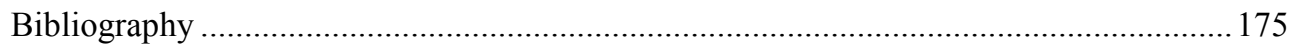




\section{List of symbols}

\section{Latin symbols}

$a$

$b$

$c$

$c_{f r}$

$c_{m}$

$c_{p}$

$c_{s}$

$c_{\text {scale }}$

$f$

$f_{H K}$

$g$

$h$

$\bar{h}$

$h_{\text {eff }}$

$h_{s}$

$h_{z}$

$k$

$k_{w}$

l

$l_{b}$

$l_{c r}$

$l_{\text {slide }}$

$m$

$m$

$m_{1}$

$m_{2}$

$n$

$\vec{n}$

$p\left(p_{a}, p_{r}\right)$

$p_{p l}$

$q$

S

$u$

Contact radius

[m]

Contact radius [m]

Constant that gives ratio $l_{b} / h$

Factor in galling model, fraction $m$ divided by $\Delta \gamma$

Compatibility parameter for adhesion between metals

Specific heat

Scaling factor

Scaling factor

Coefficient of friction

Dimensionless interface strength $\tau / k$

Gap between tool and sheet in forming simulation

Height

Dimensionless height

Effective height $\left(h_{e f f}=h_{z}-u\right)$

Separation compared to the mean summit height

Separation compared to the mean surface height

Shear strength

Wear rate

Length of part of asperity before top $\left[\mathrm{m}^{2} \mathrm{~N}^{-1}\right]$

Effective length of part of asperity behind top

Crack length

Sliding distance (for one product)

Fraction of wear volume that transfers

Tresca factor

Constant in galling model

Constant in galling model

Number of products or number of sliding tracks

Normal unit vector

Contact pressure (apparent and real contact pressure)

Normal stress on interface of plastic deforming material

Power of heat generation

Summit height compared to the mean summit height 


\begin{tabular}{|c|c|c|}
\hline$\vec{u}$ & Velocity vector & {$\left[\mathrm{m} \mathrm{s}^{-1}\right]$} \\
\hline$v$ & Sliding velocity & {$\left[\mathrm{m} \mathrm{s}^{-1}\right]$} \\
\hline$w$ & Width & [m] \\
\hline $\bar{w}$ & Dimensionless width & {$[-]$} \\
\hline$w_{\mathrm{I}}, w_{\mathrm{II}}, w_{\mathrm{III}}$ & Widths in definition of asperity with hexagon base & [m] \\
\hline$w_{\text {total }}$ & Summation of $w_{\mathrm{I}}, w_{\mathrm{II}}$ and $w_{\mathrm{III}}$ & {$[\mathrm{m}]$} \\
\hline$x$ & Coordinate direction & {$[\mathrm{m}]$} \\
\hline$x_{c}$ & Centroid ( $x$-coordinate) & [m] \\
\hline $\bar{x}_{c}$ & Dimensionless centroid ( $x$-coordinate) & {$[-]$} \\
\hline$y$ & Coordinate direction & {$[\mathrm{m}]$} \\
\hline$y_{c}$ & Centroid ( $y$-coordinate) & [m] \\
\hline$z$ & Coordinate direction & [m] \\
\hline$A\left(A_{n}, A_{r}\right)$ & Area of contact (nominal and real area) & {$\left[\mathrm{m}^{2}\right]$} \\
\hline$A_{\text {plateaus }}$ & Area of plateaus & {$\left[\mathrm{m}^{2}\right]$} \\
\hline$A_{\text {summit }}$ & Summit area & {$\left[\mathrm{m}^{2}\right]$} \\
\hline$A_{H}$ & Hamaker constant & {$[\mathrm{J}]$} \\
\hline$A_{X Y Z}$ & Area size of face $X Y Z$ & {$\left[\mathrm{~m}^{2}\right]$} \\
\hline $\bar{A}_{X Y Z}$ & Dimensionless area size of face $X Y Z$ & {$\left[\mathrm{~m}^{2}\right]$} \\
\hline$C$ & Stiffness of elastic support of asperity & {$\left[\mathrm{Pa} \mathrm{m}^{-1}\right]$} \\
\hline$C_{a b}$ & Interaction energy at a unit distance between atoms $a$ and $b$ & {$\left[\mathrm{~J} \mathrm{~m}^{6}\right]$} \\
\hline$C_{n}$ & Contact stiffness in forming simulation (penalty) & {$\left[\mathrm{Pa} \mathrm{m}^{-1}\right]$} \\
\hline E & Modulus of elasticity & {$[\mathrm{Pa}]$} \\
\hline$E^{*}$ & Reduced modulus of elasticity & {$[\mathrm{Pa}]$} \\
\hline$F\left(F_{a}, F_{n}, F_{w}\right)$ & Force (adhesion force, normal force, friction force) & {$[\mathrm{N}]$} \\
\hline$G$ & Galling impact factor & {$\left[\mathrm{m}^{2} \mathrm{~N}^{-1}\right]$} \\
\hline$H$ & Hardness & {$[\mathrm{Pa}]$} \\
\hline$I_{x x}$ & Area moment of inertia with respect to the $x$-axis & {$\left[\mathrm{m}^{4}\right]$} \\
\hline$I_{y y}$ & Area moment of inertia with respect to the $y$-axis & {$\left[\mathrm{m}^{4}\right]$} \\
\hline$I_{c y y}$ & Area moment of inertia with respect to the line $x=x_{c}$ & {$\left[\mathrm{~m}^{4}\right]$} \\
\hline$K\left(K_{\text {tool }}, K_{\text {sheet }}\right)$ & Thermal conductivity (conductivity of tool and sheet) & {$\left[\mathrm{W} \mathrm{m} \mathrm{m}^{-1} \mathrm{~K}^{-1}\right]$} \\
\hline $\mathrm{K}(n)$ & Elliptic integral of the first kind with modulus $n$ & \\
\hline$O$ & Origin in coordinate system & \\
\hline$R_{a}$ & Roughness, centre line average & {$[\mathrm{m}]$} \\
\hline$R_{a}$ & Major radius of asperity & {$[\mathrm{m}]$} \\
\hline$R_{b}$ & Minor radius of asperity & {$[\mathrm{m}]$} \\
\hline$R_{q}$ & Roughness, root mean square & [m] \\
\hline $\mathrm{T}$ & Dimensionless shear strength $k / p_{p l}$ & {$[-]$} \\
\hline$V_{\text {transfer }}$ & Volume of transferred material & {$\left[\mathrm{m}^{3}\right]$} \\
\hline
\end{tabular}


Wear volume

$\left[\mathrm{m}^{3}\right]$

W

Work of adhesion per unit area

$\left[\mathrm{J} \mathrm{m}^{-2}\right]$ 


\section{Greek symbols}

Angle in definition of asperity with hexagon base

[rad]

$\alpha$

Fraction of area into contact

$\alpha_{f r}$

$\alpha_{\text {meso }}$

Half top angle in frontal direction

[rad]

Fraction $A_{\text {plateaus }} / A_{n}$

$\alpha_{\text {micro }}$

Fraction $A_{r} / A_{\text {plateaus }}$

$\alpha_{s}$

Half top angle in sideward direction

$\beta\left(\beta_{x}, \beta_{y}\right)$

Summit radius (radius in $x$ and $y$-direction)

Surface energy of material $a$

Interfacial energy between materials $a$ and $b$

Adhesion energy $\left[\mathrm{J} \mathrm{m}^{-2}\right.$ ]

$\delta$

Summit indentation

[m]

Deformation of elastic support of asperity

[m]

Deformation at $x=0$ and $x=x_{c}$

$\delta_{0}, \delta_{c}$

Strain

Attack angle

Tilt angle of elastic support of asperity

Thermal diffusivity

Factor between plastic deformation velocity and stress

Real coefficient of friction on the interface

Poisson's ratio

Density

Number of atoms $a$ per unit volume

(Normal) stress

Ultimate strength

Yield strength

Von Mises stress

Normal stress on face $X Y Z$

Shear stress

Shear stress on face $X Y Z$

Shear stress on interface of plastic deforming material

Contact aspect ratio $b / a$

Height distribution of contact surface 


\section{Introduction}

Deep drawing is a sheet metal forming process which is widely used in, for example, the automotive industry. In this production process, a complex sheet metal product is formed starting from a plane sheet. Some examples of these products are car body parts, shaver caps, lemonade cans, pans and pan lids. An impression of the deep drawing process is given in figure 1.1. In the cross section, the deforming sheet metal and the process forces that act on the tools are shown. The die and the punch are the tools which contain the information of the desired geometry of the formed product. The die is the static tool which supports the sheet; the punch is the moving tool which presses the shape into sheet. The blank holder clamps the sheet on the die in order to control the sheet flow during forming. In this manner wrinkling of the workpiece can be avoided.

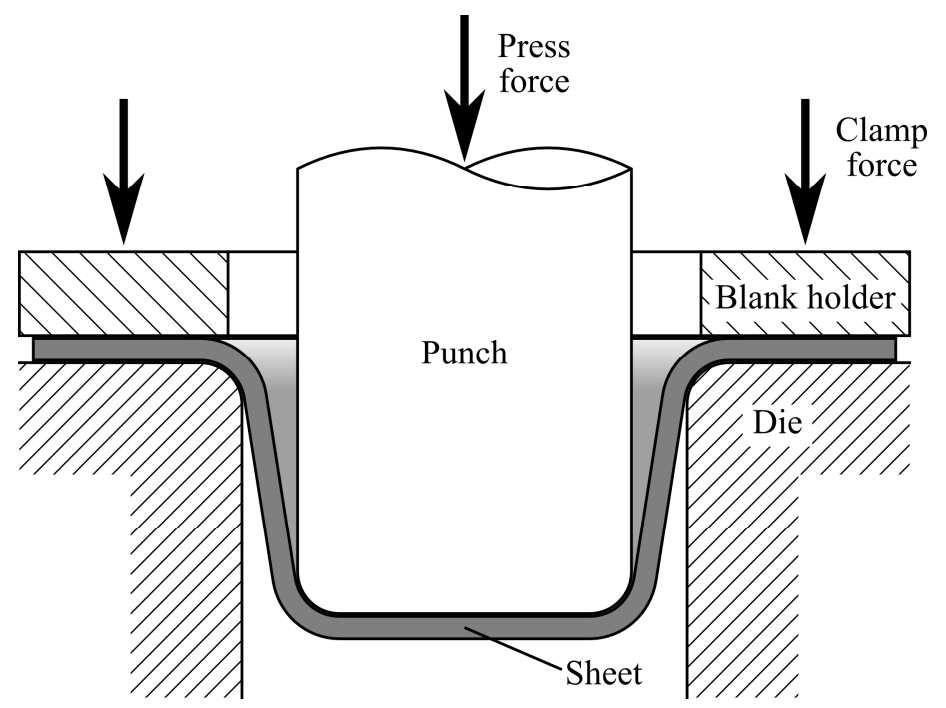

Figure 1.1. Deep drawing process.

The production process can be characterized by complex and expensive tooling and is therefore mainly used for mass production. Because of the high costs, the tools have to meet high requirements, as will be described below.

Firstly, the tools should be able to create the geometry of the sheet metal product within its tolerances. This requirement is responsible for the high costs of the tools. Nowadays, deep drawing processes are typically developed using FEM simulations of the process. After manufacturing, the tools often have to be polished and refined by hand. This is more or less a trial and error process based on the experience of the tool workshop. By improving the accuracy of the FEM simulations, the trial and error process is reduced. 
Secondly, the lifetime of the tool as related to geometrical product deviations is of importance. Wear causes geometrical deviations of the original shape. Beside deviations which are immediately related to tool shape deviations, friction can change due to surface defects of the tool. These defects can influence the product geometry, because of the reduced control on the friction. Further, these surface defects can damage the product surface. Due to the high costs of the tools and the high investments of a press shop, the lifetime of the tool is of great importance.

A number of reasons are known that limit the lifetime of the tool. Below, some of them are given.

- Wear of the tool which results in shape deviations of the product.

- Dusting and flaking. These are processes which occur with the use of zinc coated material. In the case of dusting, zinc powder comes loose from the zinc coated layer, and pollutes the tools. Flaking is another case of pollution, where flakes come loose from the zinc layer due to inadequate adhesion or cohesion in the zinc layer itself. These forms of pollution may damage the tools and because of that effect the subsequent products may be damaged. If dusting or flaking happens in industrial practice, the production process has to be stopped from time to time to clean the tools.

- Galling, a phenomenon caused by material transfer from the sheet to the tool.

In this thesis, the focus is on the last phenomenon, galling, and will be discussed in more detail in the following section.

\subsection{Galling}

Galling is a known failure mechanism in sheet metal forming processes. It is defined as the mechanism in which material transfer takes place from the sheet to the tool to form lumps, whereby the lumps now attached to the tool will subsequently cause scratching into the sheet. This can result in a severely scratched product. The effect of this phenomenon on the surface quality is shown in figure 1.2. 


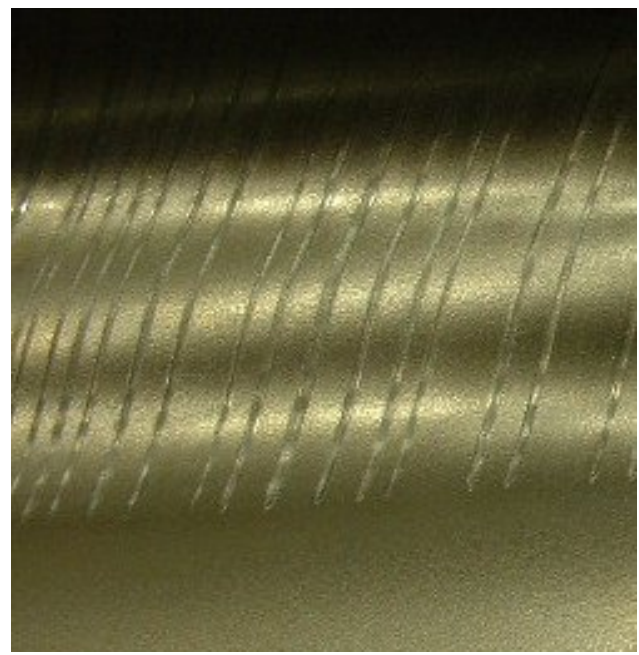

Figure 1.2. Example of severely scratched product due to galling.

Galling is a form of failure in which a number of phenomena play a role. The material transfer from the sheet to the tool indicates adhesive wear at the interface between the tool and the sheet material, which is stronger than the sheet material itself. In this way the lump can grow. Further, the transferred material has undergone work hardening or is composed of an oxide of the sheet material, which is harder than the sheet material itself [17]. Due to the increased hardness, the lump (which in fact consists of sheet material) is to a certain extent able to sustain the stresses generated during scratching.

On deep drawing products small scratches can often be observed. Due to galling, these scratches become more serious. If the scratches reach a certain level, the products are labelled as scrap. If these scratches are due to the condition of a tool, this tool has to be reworked or replaced by another. It is difficult to give a general limit for an acceptable amount of galling behaviour. According to De Rooij [39] this limit depends strongly on the application of the product and on the paint procedure which is used after the forming operation. Typical depths of defects that are still visible after a paint procedure are in the order of $20 \mu \mathrm{m}$.

\subsection{Tribological system}

To investigate the tribological behaviour of a contact, usually a tribological system will be defined as proposed by Czichos [13]. In general a system is defined as a structure and its function. The structure is built of a set of elements, usually four elements: the two contact bodies, the interface between the contacting bodies and the environment. The function is connected to the system inputs and outputs. For a tribological system, typical inputs are 
load, temperature, velocity and type of motion. Friction and wear are the outputs. In the context of the deep drawing production process, the contact bodies are the sheet and the tool (for example the die), see figure 1.3. The interface is most frequently a lubricant and can be absent in an unlubricated tribological system. The lubricant can be a deep drawing oil, but also a prelub which combines the functions of a conservation oil and a deep drawing lubricant. In the case of a lubricant-free deep drawing process, the available surface layers of the contact bodies have to fulfil the interface functions on their own. The environment is assumed to be the atmosphere of the production hall. To define the environment in this manner, special cases are excluded, such as hot forming.

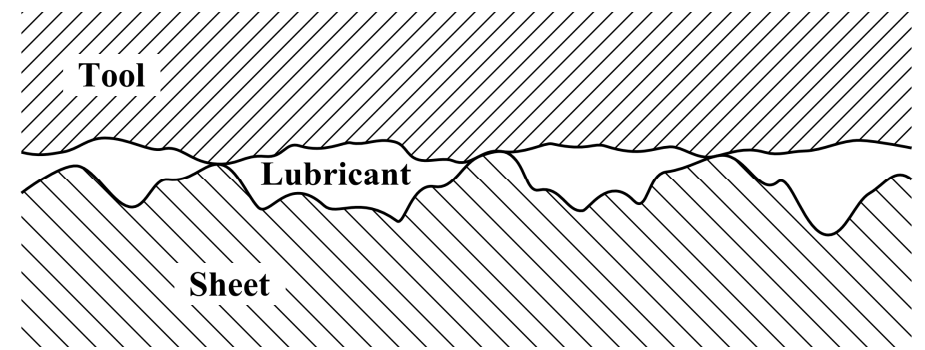

Figure 1.3. Elements of tribological system. The tool and sheet are the contact bodies, the lubricant as interface. The fourth element, the environment, is left out.

Some assumptions are made in the tribological system used in this thesis:

- The properties of the tool do not change, so, the roughness is constant. The only shape deviations in this system are due to material transfer, the galling phenomenon.

- The sheet has a fresh surface and is still not work hardened, because it is not runin. Therefore, the sheet is relatively soft and treated as a perfect plastic material.

- In the contact, lubricant is present.

The proposed system deviates from the more common one, where the surface does not plastically deform, although some plastic deformation of the asperity may occur. That type of surface is usual (and at least desirable) in machine element contacts. In the proposed system the sheet has a fresh surface, which is softer than the tool.

Two types of plastic deformation of the sheet can be distinguished. One is the deformation of the surface as a result of contact stresses. This type of deformation is very local and can be derived within the envelope of the tribological system. The other is the deformation of the bulk of the sheet, which is, after all, the purpose of the forming process. This is a deformation on a larger scale than the system envelope. This deformation may influence the contact behaviour and can therefore be a useful input of the system. 
Sengupta [43] shows different zones in a deep drawn product, which undergoes different modes of stresses and plastic strains. These zones will be named here below and shown in figure 1.4.

1) At the punch nose, a biaxial stretching of the sheet.

2) On the punch corner radius the sheet material is stretched and bent around the corner radius.

3) Between the die and the punch is a region where the sheet material is not supported. Here, a pure tensile straining takes place.

4) At the die radius is a stretch bending state.

5) At the blank holder region the sheet is radial drawn, accompanied by circumferential compression.

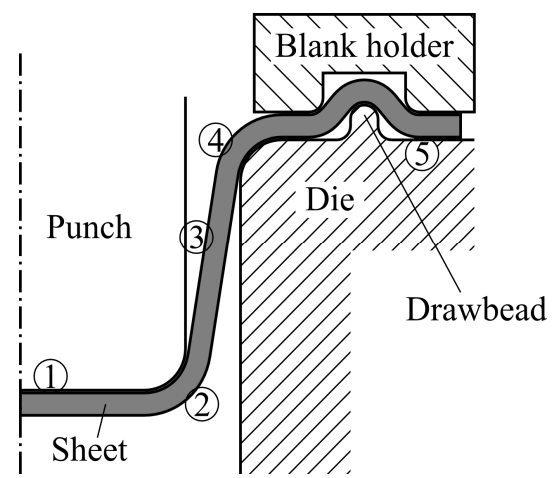

Figure 1.4. Deep drawing process with different zones. Zone 5 may contain a drawbead.

The zones at points 1 and 2 are not very sensitive to galling due to the low sliding velocities. The low velocity has two effects: little heat generation in the contact (so, little or no lubricant failure) and little sliding distance. The unsupported zone of point 3 does not have any galling risk, due to the absence of contact.

The zones given at points 4 and 5 are the most galling sensitive, due to the combination of contact pressure and sliding velocity. At point 4 the sheet material is bent over the die radius by the punch force. According to numerical calculations by Sniekers [44] the pressure in this zone is not uniformly distributed, but concentrated at the entrance and the exit of this zone, so the peak levels of the contact pressure can be much higher as the mean level. In the zone indicated with point 5 , the pressure increases due to the decreasing contact surface in the blank holder and as a result there is a decreasing sliding velocity. This decreasing contact surface is a result of the decreasing outer radius of the blank and the thickening of the blank at the outer radius due to circumferential compression. The real contact pressure at the outer radius of the blank is compared with the mean contact pressure 
at the blank holder by Emmens [14]. In this work, it is shown that the real contact pressure is sometimes twice as much as the mean contact pressure. The effects presented by Sniekers and Emmens can be seen to some extent in figure 2.2.

Within the blank holder zone a special region can sometimes be found which is not mentioned in [43]. That is the drawbead, which is a ridge in the blank holder zone, see figure 1.4. In addition to the radial elongation and the circumferential compression here, bending also occurs over this ridge. This results in local high pressures and is therefore a risky location in the product from the viewpoint of galling.

\subsection{Objective of the research: development of a Galling Performance Indicator}

The objective of the research is to develop a Galling Performance Indicator (GPI) from which the galling tendency of an aluminium and zinc coated sheet material in forming operations can be estimated. The GPI will be developed in such a way that it can be used as a post processor for FEM simulations of the deep drawing process. In this way the process can already be studied in terms of surface quality in the simulation stage. This reduces trial and error costs. The idea is to formulate this indicator in such a way that it predicts the galling behaviour on the basis of two input categories. These categories are:

- Properties like the surface properties of the materials in contact (sheet and tool), coatings (if present), roughness, the lubricant and the temperature. Although, in the tribo system, these properties act on a small scale (roughness scale), the quantities belonging to these properties are more or less constant over tool and sheet.

- Quantities as a result of the forming process, like the stresses, strains and sliding velocities. In general, these quantities are obtained by FEM simulations and are determined on a larger scale than the roughness.

These inputs will be used for the formulation of a tribo system, as shown in figure 1.5. The calculation steps in the tribo system are marked within the dashed line. The contact state will be determined. On the basis of this state the amount of material transfer will be defined. With the transferred material, the new surface geometry can be predicted. 


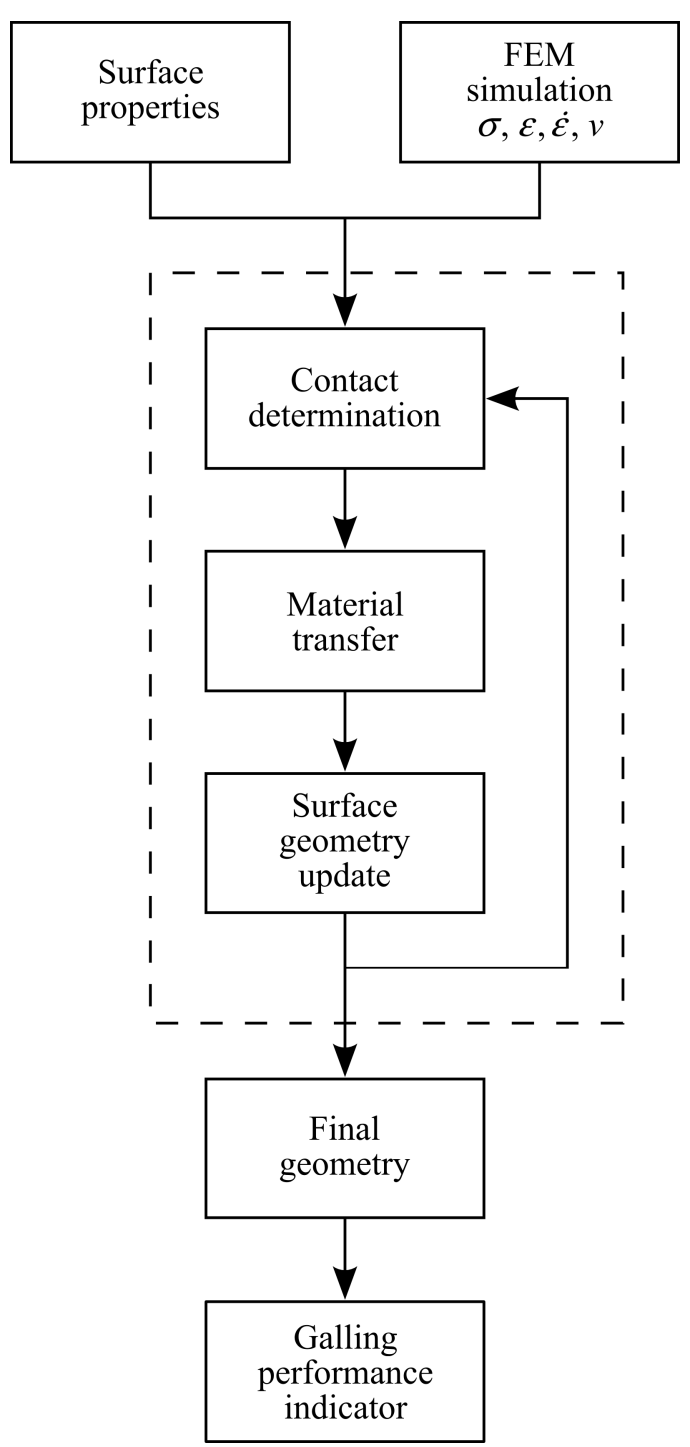

Figure 1.5. Strategy to determine the effect of galling.

The research will be restricted to a selection of tool and sheet materials and some types of lubricant. Although the results of this work may cover more general results, the focus is on one type of aluminium sheet and zinc coated steel sheet. The aluminium sheets, divided into two types of surface structures are AA5182 EDT and AA5182 MF. The zinc coated steel sheet is DX54D Z EDT, which is a sheet with a galvanized zinc layer. The lubricant that is used depends on the type of sheet material. In the case of zinc coated sheet, the 
lubricant is Quaker N6130 and Dry Coat DC2 for aluminium. The tool material is restricted to tool steel WN 1.2379.

The contact bodies are assumed to be at room temperature. Isothermal behaviour is assumed, both for the environment temperature and the bulk temperature of the tools and sheet. The effect of temperature rise due to plastic forming will be ignored.

\subsection{Outline of this thesis}

To derive a method to estimate the galling tendency a number of steps are performed. In chapter 2 existing contact and galling models are presented which give a description of the contact that is relevant in the case of deep drawing with respect to galling. In chapter 3 experiments are described that reproduce a single asperity contact between a tool asperity and sheet in the deep drawing process. The mechanism of material pick-up will be investigated for different combinations of tool and sheet materials. The results of this investigation are used in chapter 4 for the formulation of a material transfer model. The model that is presented in chapter 4 gives the lump growth on a single asperity and is based on the wear behaviour in the contact between tool and sheet, adhesion properties and the mechanical stability of the lump. In chapter 5 this model is implemented for the multi asperity case. The galling tendency of a real surface can be determined on the basis of a known tool roughness topography and based on contact parameters like the apparent contact pressure and sliding velocity. On the basis of this multi asperity model a GPI is formulated. Finally, in chapter 6 the main conclusions and recommendations are given. 


\section{Contact in deep drawing and galling}

\subsection{Contact scales}

The contact in the deep drawing practice is formed by a sheet and a tool. The tool has a smooth hard surface which is in contact with the sheet. The sheet has a soft, relatively rough and fresh surface, because in deep drawing a new sheet is placed every time against the tool surface. Further, due to plastic deformation, oxide layers on the surface will be broken. These properties contrast with most contact situations in mechanical engineering. Mostly, contacts in machine components are formed by surfaces which are run-in.

The contact situation can be divided into different length scales. Every scale has its own characteristics and plays its own role in the case of deep drawing. Following De Rooij [39] three scales will be distinguished. These different scales of magnification are given in the following sections. These scales are divided as macro contact, meso contact and micro contact. The macro contact is the contact as seen on a scale visible to eye. The meso contact is the contact of the flattened sheet roughness against the flat tool. The micro contact is the contact of tool asperities in contact with a plateau (a flattened region) on the sheet. In [39] the scales are named differently, namely macro, micro and asperity scale.

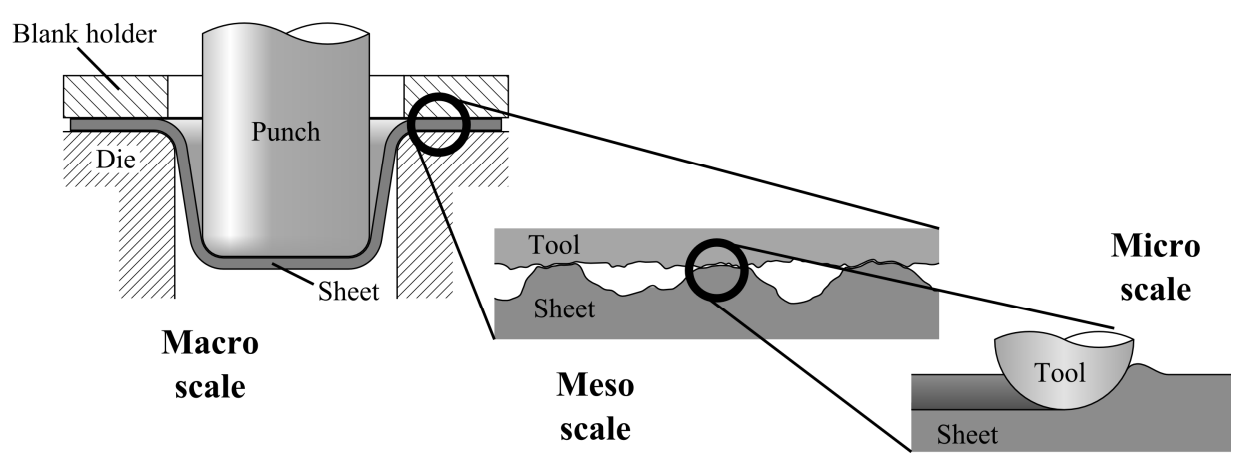

Figure 2.1. Different contact scales.

\subsubsection{Macro scale}

The macro contact scale is the contact scale where both the tool and the sheet surface are considered as smooth. Here, the focus is on the physical quantities of the forming operation assuming the nominal dimension of the tools and the sheet. From this level the contact pressures, stresses and strains are determined, ignoring the roughness from both the tool and the sheet. This is the scale of FE-analyses where physical quantities like sliding 
velocities and stresses can be calculated. Other issues can be the determination of process forces and the check whether a product can be formed according to the forming limit diagram [3].

In the scope of this thesis, this scale is important to get physical 'bulk' quantities which can be used as an input on smaller scales. The contact pressure calculated on the macro scale can be used as the apparent contact pressure on the meso scale. The same strategy can be used for stresses and strains. A local stress state on the macro scale can be used as the mean stress state on the meso scale. The strain rate calculated on this scale can be used for calculating the effective hardness on a smaller length scale. This is important for the flattening of the sheet roughness as is shown in [28] and [48]. As an example, some results of a deep drawing FEM simulation are shown in figure 2.2, calculated with DiekA ${ }^{1}$ using 3D Discrete Kirchhoff Triangle elements, a type of sheet elements. In this figure contact pressures and effective strain are presented at both the top and bottom side of the product. The product is a cup with a radius of $25 \mathrm{~mm}$. The figure shows the forming state after a stroke of the punch of $25 \mathrm{~mm}$. The initial blank has a radius of $50 \mathrm{~mm}$ and a thickness of $0.8 \mathrm{~mm}$. The contact pressures are determined using the reaction stresses of the contact elements, because the sheet elements calculate only the in-plane stresses of the sheet. The calculation of the contact stress is given in more detail in section 2.2.1. From figure 2.2 it becomes clear that on the die radius some pressure peaks appear and this region has a higher level of strain.

On this scale different zones can be distinguished as discussed in section 1.2.

${ }^{1}$ DiekA is an in-house finite element code for forming simulations developed at the University of Twente, www.dieka.org. 

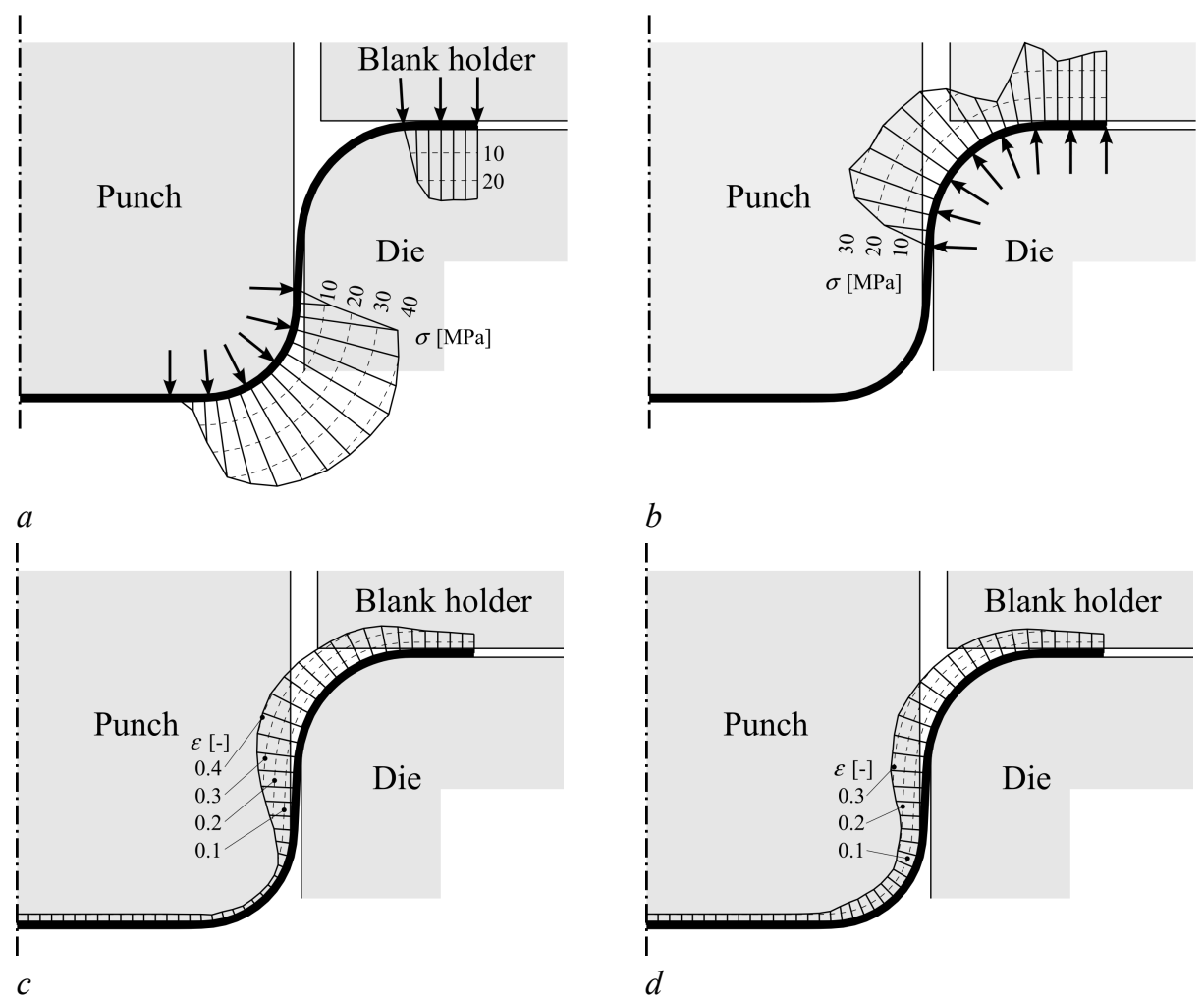

Figure 2.2. Results of a finite element calculation. It shows is the contact pressure distribution at the top (a) and the underside (b) of the sheet and the equivalent strain at the top (c) and underside (d).

\subsubsection{Meso scale}

The meso scale is the scale on which roughness can be observed. On this scale, the sheet surface shows a higher roughness than the tool surface.

The focus on the meso contact scale is on the flattening of the sheet roughness. The tool on this scale is still relatively smooth compared to the sheet roughness. Due to contact pressure in combination with strain of the bulk material, the smooth hard tool flattens the softer and rougher sheet material. This flattening is dependent on the contact situation. Sengupta [43] shows the effect of lubrication. In the case of lubrication, the load is (partially) carried by the enclosed lubricant in the contact, which in turn distributes the load over the peaks and the valleys of the roughness. Wilson and Sheu [48] show that the flattening of the sheet roughness is a function of the difference of the direct contact pressure of the asperities and 
the lubricant pressure. Besides, in [48] the effect of a one-dimensional bulk strain is shown. In [28] the effect is shown of different strain situations, in the case of a one-dimensional roughness, with the principal strain in plane of the sheet in the direction of the asperities and perpendicular on the asperities. From this, it follows that the contact area on the meso scale is very sensitive to the lubrication and the plastic deformation state of the bulk material.

\subsubsection{Micro scale}

On the meso contact scale the flattening of the asperities can be observed and the load is carried by the formed plateaus and, depending on the operational conditions, the lubricant. Zooming in on a single plateau, the roughness of the tool can be observed. This scale will be called the micro scale. The roughness on this scale is usually formed by grinding or polishing the tool. Due to the relative motion of the tool surface in relation to the sheet surface and the difference in hardness, the roughness of the tool scratches into the sheet metal. This roughness level is of importance for galling. Under certain conditions sheet material transfers from the plateaus to the tool asperities. When material transfer takes place, lumps grow on the tool summits. As a result these lumps scratch into the sheet material of the formed product. This scratching behaviour has two effects:

- The friction behaviour in the contact between the tool and the sheet changes. According to [34] and [39] the friction gets a larger standard deviation. This means worse control of the sheet metal forming process.

- If the lumps grown on the tool surface are too large, the sheet material becomes damaged too much. In this way a protective layer can be locally changed or removed, like a zinc layer in the case of zinc coated sheet. Another aspect is the aesthetic one. If the scratches are too deep, a paint layer is not able to fill the grooves in the sheet surface and scratches remain visible.

\subsection{Contact models}

In this section some relevant contact models on the different scales are presented. In section 2.2.1 a contact model used in FEM calculations is presented. This model will be discussed, because it is relevant for obtaining and interpreting the data of the FEM calculations. In sections 2.2.2 and 2.2.3 models are presented which are more focussed on the details of the contact, which cover the more relevant properties needed to explain and calculate the galling effect. 


\subsubsection{Macro scale}

In this section the contact modelling on the macro scale will be discussed. On the macro scale both the tool and the sheet surface are considered as smooth, as already mentioned in section 2.1.1. The focus in this thesis is on the roughness scale, so the contact on macro scale is only of importance for obtaining mean values on the smaller scales. These values can be obtained by FEM calculations of forming simulations. Some aspects of contact in these FEM calculations are given here.

In forming simulations three areas can be distinguished, each having its own characteristics. Hereafter these areas will be given together with the parts that are used in the case of deep drawing, as an example.

1) The workpiece: the blank.

2) The tools: the punch, die and blank holder.

3) Contact: the connection between the workpiece and the tools.

The workpiece is modelled with an element type that is able to describe plastic deformation. A lot of material models are available that can be the basis of the element formulation, like for example rigid-plastic or elastic-plastic models. Once the element is formulated and used in a FEM calculation, the material and mechanical behaviour is defined. Within the scope of determining the contact situation, only calculated values at the surface of the material in the contact region are of interest. Normal pressure equals the stress perpendicular to the workpiece surface; friction stresses equal the tangential stresses parallel to the surface.

Problems arise in the case of planar elements, which are used in, for example, deep drawing processes. The number of stress components formulated in this type of element is reduced. This type of element takes into account membrane stresses and additionally bending and transverse shear stresses. The stress components given by all these types of elements are the in-plane normal stresses and shear stress. In general this is given as $\sigma_{x x}, \sigma_{y y}$ and $\tau_{x y}$, with the $z$-axis perpendicular to the surface. With this type of element, it is not possible to get the normal pressure and shear stresses on the surface in the contact. Another strategy has to be applied, which will be given hereafter.

The modelling of the tools has the function of giving geometrical constraints to the workpiece. These constraints are a function of location, time and sometimes of physical quantities like pressure and temperature. The simplest case is the situation where the constraints are only a function of location and time. In this case only the geometry of the contact region has to be modelled. This can be done with a mesh or in the case of simple geometries by an analytical description. In the case of more complex constraints, for example elastic tools, the body of the tool has to be modelled as well with elements that describe the material of the tool. 
The key to obtaining the contact pressure in the case of planar elements is found in the contact elements. In the case where the distance between a workpiece node and the tools is above a certain level, the elements do nothing. Below this distance, the element determines the contact pressure using a penalty function, defined as:

$$
\sigma_{n}= \begin{cases}C_{n} g & \text { if } g \leq 0 \\ 0 & \text { if } g>0\end{cases}
$$

The penalty $C_{n}$ is a fictive stiffness of the contact; $g$ is the gap between the workpiece node and the tool. This is fictive, because a surface in geometrical sense cannot have stiffness; this is only the property of a body. In the case of penetration of the workpiece in the tool (finite $C_{n}$ value), the contact element generates a contact pressure. Depending on the $C_{n}$ value used, the contact pressure changes [8], as can be seen from equation (2.1). In the case of an infinite value of $C_{n}$ no penetration of the workpiece into the tools is present, but this gives numerical problems. In practice, a compromise between stiffness and numerical stability has to be found. Although the stiffness is fictive, the contact pressure generated through this method is an estimation of reality, because the generated reaction stresses form the workpiece such that the tool constraints are approximated. The better this approximation is, the higher the accuracy of the contact stresses found in this manner. The influence of the penalty $C_{n}$ is shown in figure 2.3 . The same simulation is performed three times, only the contact stiffness is altered. According to this calculation the pressure distribution becomes flatter in the case of a lower stiffness. So, as long as a higher stiffness gives no numerical problems, more details of the contact pressure are obtained. This may be important to find local pressure peaks. Typically, pressure peaks at the beginning and end of the die radius region are clearer in figure $2.3 \mathrm{c}$ than in figure $2.3 \mathrm{a}$. Compared to the results presented in figure $2.3 \mathrm{a}$ the local pressure peaks on the die radius in figure $2.3 \mathrm{c}$ are about $15 \%$ larger, on the blank edge even $20 \%$, the pressure valley on the die radius is about $50 \%$ lower. From these values it may be concluded that the stiffness conditions influence the results that are obtained by FEM calculations. So, using contact data obtained from FEM calculations, one should be aware of the influence of the contact stiffness penalty. 


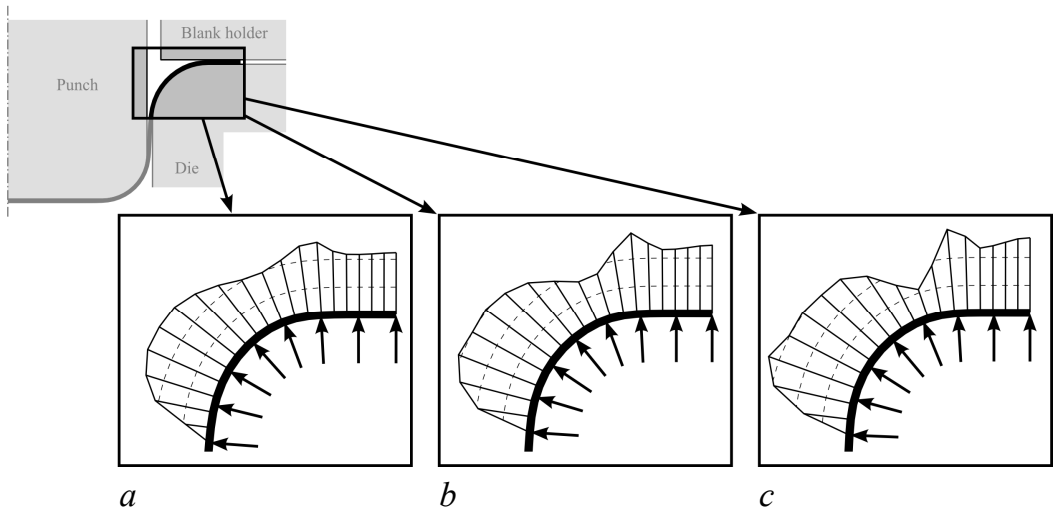

Figure 2.3. Influence of $C_{n}$ in the contact model on the contact pressure of the die. The pressure is presented as the graph perpendicular on the sheet surface. The values of $C_{n}$ are 100 (a), 200 (b) and $400 \mathrm{~N} / \mathrm{mm}^{3}$ (c).

The shear stress on the sheet surface, that is the friction stress, is also determined using the contact elements. When the shear stress is determined, using Stribeck frictional behaviour curve or the Coulomb friction model, it is strongly related to the normal stress. That means that the influence of the contact stiffness penalty also acts on the magnitude of surface shear stress.

\subsubsection{Meso scale}

As already mentioned in section 2.1.2, the meso scale is the scale on which the roughness can be observed. The roughness in combination with the mechanical properties of the contact bodies determines the contact behaviour. On the meso scale only the roughness of the sheet material is taken into account and the tool is supposed to be flat. The sheet is relatively soft compared to the tool. Because the sheet is relatively rough and soft compared to the tool, plateaus are formed on the sheet during loading of the contact between these bodies, due to plastic deformation of the sheet. An example of the geometry of a sheet surface is shown in figure 2.4 . 


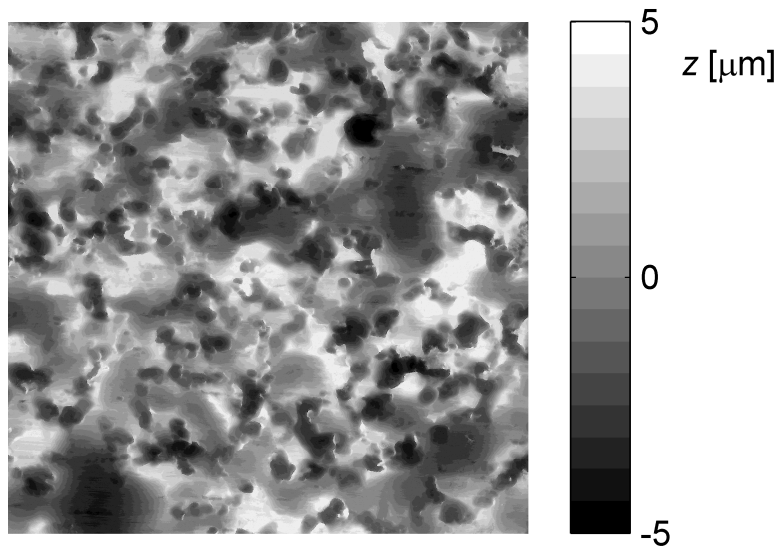

Figure 2.4. Surface geometry of sheet metal with an EDT texture, given as a height map. The area is $1 \mathrm{~mm}^{2}$.

The size of these contacts can be calculated using force equilibrium integrating the contact pressure over the real contact and using some assumptions. First, the tool is assumed to be rigid and ideally flat and the sheet deforms plastically. The second assumption is that the real contact pressure $p_{r}$ equals the hardness $H$, so:

$$
p_{a}=\alpha H
$$

In this relation $p_{a}$ is the apparent contact pressure and $\alpha$ the fraction of area into contact defined as $A_{r} / A_{n}$, with $A_{r}$ and $A_{n}$ respectively the real and the nominal area of contact. From relation (2.2) and $\alpha$ given as:

$$
\alpha=\frac{A_{r}}{A_{n}}=\int_{h_{z}}^{\infty} \phi(z) d z
$$

the separation $h_{z}$ can be calculated. Here, $\phi$ is the height distribution function of the sheet surface and $h_{z}$ the separation of the surfaces, defined as distance between the tool surface and the mean surface height line of the sheet. The sheet surface above the level $h_{z}$ forms the contact as depicted in figure 2.5. The material above the level $h_{z}$ is pressed into the sheet surface.

According to Pullen and Williamson [37] this relation is only true if the different contact spots are well separated from each other and operate independently of each other. In [37] is shown that normally contact spots are not independent of each other. Due to the indentation of the surface fraction that is in contact, the surface fraction that is not in contact rises with a constant value $u$, see figure 2.5. The figure shows at the dark areas the material that is 
pushed into the rough surface and is responsible for the surface rise of the contact fraction that is not in contact. The constant rise of the valleys is used to derive the following relation:

$$
\frac{p_{a}}{H}=\frac{\alpha}{1-\alpha}
$$

To calculate $\alpha$ in relation (2.4), $h_{z}$ in relation (2.3) has to be replaced by $h_{\text {eff, }}$, with $h_{\text {eff }}=h_{z}-u$, the separation compensated by the rise of the valleys.

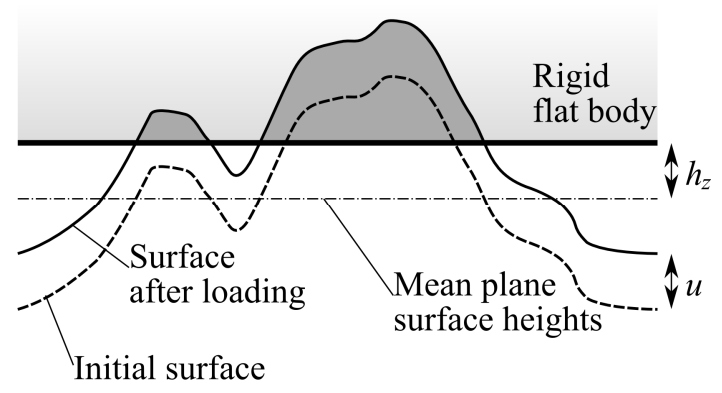

Figure 2.5. Rise of soft rough surface loaded by a rigid flat, after [37].

Because of the limitations of the model of Pullen, Westeneng [47] developed an extended contact model. Aspects which are covered in the model of Westeneng and not in the model of Pullen are the following: asperity persistence (i.e. the resistance against deformation at higher loads), work hardening and tensile bulk deformation. The drawback of the Westeneng model is the complexity of the model. This complexity deals with the calculations as well as how to obtain parameters of the model, like the persistence parameter.

\subsubsection{Micro scale}

On the micro scale the roughness of the tool is of interest. As shown in figure 2.1 on this level, the tool roughness is in contact with the flat plateaus that are formed on the sheet surface. In the existing galling models, presented in section 2.3, the contact is based on summit contact models. These models use the following steps:

1) The roughness is determined, specially the summits.

2) The contact is determined. This is related to the summits in contact.

3) The wear regime of every summit is determined. This is related to the galling behaviour. 
Below, these steps will be discussed. In figure 2.6 an example of the geometry of a tool surface is shown.

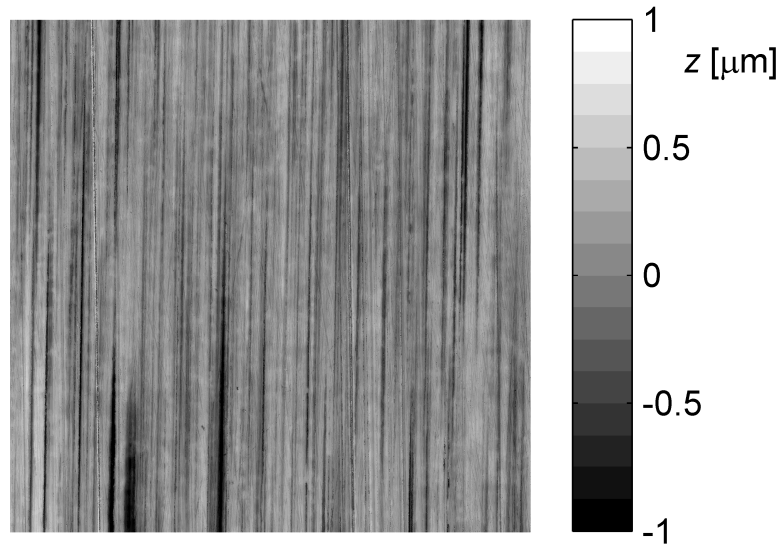

Figure 2.6. Surface geometry of a tool with a grinded surface, given as a height map. The area is $1 \mathrm{~mm}^{2}$.

\section{Determination of roughness}

Because of the random nature of roughness, it is very difficult, if not impossible, to describe the exact surface geometry. To describe the phenomena in the contact, there are some alternatives to characterise the surface. This can be done by:

- Discretization of the surface. In general a part of the surface is taken, that covers the relevant properties. The wavelengths that can be described are limited on the one hand by the spatial resolution and on the other hand by the measurement domain. The discretization of the surface is done by surface measurement equipment, which measures the local surface heights on a line or on a grid on equidistant points. In this way a vector with profile data or a matrix with surface data is obtained. Examples of surface measurement equipment are an interference microscope and a confocal microscope.

- Describing the surface by a reduced data set, for example a set which contains only basic data of the summits, like the height and radius of every individual summit. In this and the former method, generally only a part of the contact surface is covered by the data and it is of importance that this data is representative for the whole contact area.

- Describing the surface statistical parameters, for example the height density function, the $R_{a}$ or $R_{q}$ value and the summit density. 
These methods describing the surface are not completely independent, although the implementation in contact models may be very different. Describing the surface in a few scalar parameters or an algebraic height distribution, like the Gaussian distribution, is mainly used for algebraic studies and simple sensitivity studies. The discretized surfaces are used in numerical models, like finite element or boundary element analysis. In practice the discretized surface data are used to construct the other types of surface description. For example, the height distribution function or a set of summits is constructed from the discretized data.

The galling models given in section 2.3 are based on summit contact, using spherical or elliptical summits. In the initiation model described in section 2.3.1, Van der Heide [20] used a set of summits, containing the height and radius of every summit. In the lump growth model described in section 2.3.2, De Rooij [39] used a fixed radius for every summit combined with a summit height density function.

To use data of real tool surfaces in the galling models, some data conversions have to be carried out. First discretized surface data has to be generated by a surface measurement. From this discretized surface the summits have to be extracted. In [20] and [39] this is performed by the nine-point criterion [16]. In this method points are defined as a summit if its height exceeds the height of its eight direct neighbour points. The next step is to define the radius of the summit using the finite difference method. The advantage of this method is its simplicity. The drawback is the scale dependency.

\section{Determination of contact}

To determine the contact situation, the basic physical principles at the micro scale are similar to the meso scale. The difference is the way of modelling. The contact is determined on the basis of force equilibrium and plastic deformation of the surface, but in this case on the basis of summit contact.

To fulfil the requirement of equilibrium, the summation of each individual summit has to be equal to the total normal force. In the case of a spherical summit, assuming that contact pressure $p_{r}$ equals the hardness $H$, the force on a summit with radius $\beta$ is:

$$
F_{n}=\pi a^{2} H=2 \pi \beta \delta H
$$

In this relation $a$ is the radius of contact and $\delta$ the indentation of the rigid summit in the plastic material of the counter surface. The last term of relation (2.5) is based on a paraboloid approximation of a sphere. Relation (2.5) holds for the situation that is equal to an indentation, that means that in all directions of the summit there is contact. In the case of scratching and plastic deformation of the counter surface, the contact is concentrated at the front side of the summit, see figure 2.7. In that situation, relation (2.5) has to be corrected for the reduced real contact area. For plastic material behaviour and keeping the indentation 
constant, relation (2.5) has to be multiplied with a correction factor of 0.5 . In the case of elastoplastic material behaviour this correction factor is in between 0.5 and 1 .

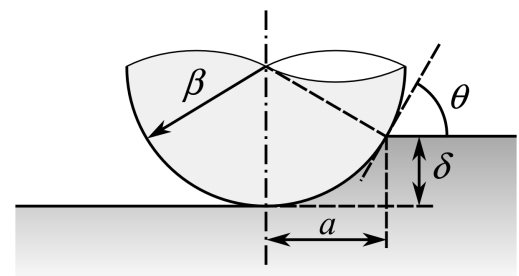

Figure 2.7. Definition of quantities related to a summit.

The summation of the load carried by all the summits gives:

$$
F_{n}=\sum_{i} F_{n i}=2 \pi H \sum_{i \in \Omega} \beta_{i} \delta_{i}=2 \pi H \sum_{i \in \Omega} \beta_{i}\left(s_{i}-h_{s}\right)
$$

Here, $s$ is the height of a summit, $h_{s}$ the separation between a plastic sheet plateau and the mean plane of the summit height of the tool surface, see figure 2.8. $\Omega$ is the set of summits that are in contact. In the case of a given surface geometry, a relation is obtained between $F_{n}$ and $h_{s}$, so the separation between the surfaces of the tool and sheet can be calculated for a given normal force. This is an iterative calculation, because the set $\Omega$ is not known beforehand. Once $h_{s}$ is known, each individual $\delta_{i}$ (equal to $s_{i}-h_{s}$ ) as well as the attack angle $\theta$ of every summit is known, see figure 2.7. In a comparable manner, this is possible with a summit height density function assuming a constant radius. The attack angle $\theta$ will be used in wear and galling models and is defined as:

$$
\theta=\arctan \left(\frac{\sqrt{\delta(2 \beta-\delta)}}{\beta-\delta}\right)
$$

Sometimes, the summit geometry is defined using two radii in perpendicular directions. This gives an extra degree of freedom, as a result of which it becomes possible to formulate a summit with an elliptic contact spot. Equation (2.5) changes to:

$$
F_{n}=\pi a b H=2 \pi \sqrt{\beta_{x} \beta_{y}} \delta H
$$

In equation (2.8) $a$ and $b$ are the radii of the contact ellipse in, respectively, the sliding direction and perpendicular to it. The radii of the summit are given by $\beta_{x}$ and $\beta_{y}$, where $x$ gives the sliding direction. In the models derived in this thesis, in cases where an asperity is defined as an ellipsoid, it will be done by using two radii as given here. 


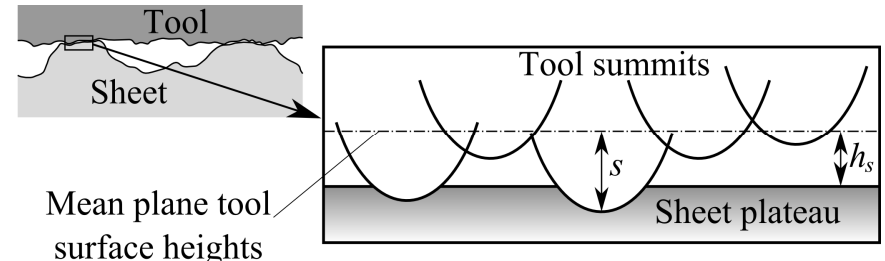

Figure 2.8. Definition of summit height and surface separation on micro scale.

\section{Wear regime}

The wear regime is closely related to the contact situation. The contact as given above can be used as a starting point for determining the wear regime. The wear regime is related to galling as used in the models given in section 2.3.

The relation between the contact situation and the wear mode of a spherical asperity is shown by Hokkirigawa and Kato [24]. On the basis of experiments and a comparison with the slipline models of Challen and Oxley [10] a wear mode diagram is constructed, which gives the wear mode in the case of a given degree of penetration and the shear strength at the contact interface. In this diagram, three wear modes are distinguished:

- Ploughing: material is displaced from the wear track to side ridges, no material is removed.

- Wedge formation: formation of a wedge of material in front of an asperity.

- Cutting: removal of material in the form of ribbon-like wear debris.

In [24] the wear modes for a spherical asperity are compared with results obtained by a set of $2 \mathrm{D}$ slipline models presented in [10]. Due to the 2D nature of the slipline models, the contact situations of the models don't fully agree with the experiments. Nevertheless, the results of the slipline models and the experiments have useful similarities. The models are shown in figure 2.9. In the slipline models, some differences can be observed compared to a contact with a spherical asperity. The spherical asperity is changed into a wedge shaped one. Besides that, the wear modes are slightly adapted. Because of the $2 \mathrm{D}$ nature, it is not possible to form side ridges. The ploughing mode in the $2 \mathrm{D}$ situation is replaced by a rubbing mode, so the material can only flow under the wedge, as can be seen in figure 2.9a. The wedge mode is explained by a model, called the wear model. In fact, the model is not a correct slipline field, because it does not show a steady field, but it is able to describe the instationary wear behaviour. The cutting model is not really different in a 3D situation. 


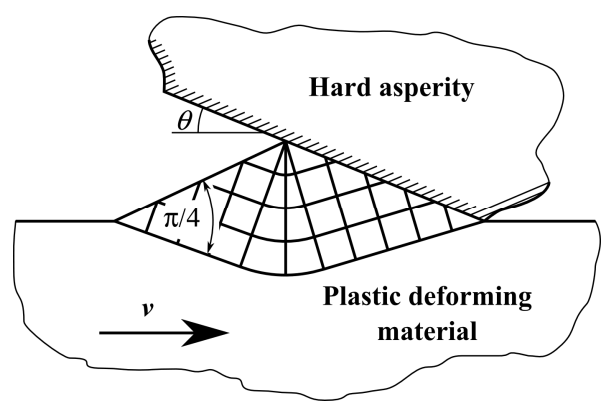

a

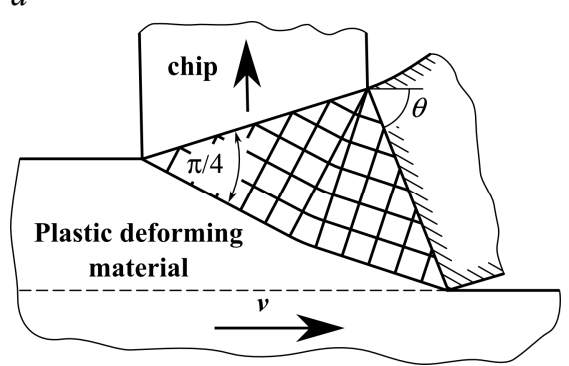

c

Figure 2.9. Slipline wear models according to [10] with the modes rubbing (a), wear (b) and cutting (c).

On the basis of this model the wear regimes are determined by two variables: the attack angle $\theta$ and the dimensionless shear strength of the interface $f_{H K}$. The definition of $f_{H K}$ is given by $f_{H K}=\tau / k$, with $\tau$ the shear strength of the interface and $k$ the shear strength of the bulk material of the plastic deforming body.

According to [39] the regimes are separated by two functions, see figure 2.10 .

The transition between the cutting regime and the others is given by:

$$
\theta=0.25 \cdot\left(\pi-\arccos f_{H K}\right)
$$

and between the ploughing and wedging regime by:

$$
\theta=0.5 \cdot \arccos f_{H K}
$$

Relation (2.9b) follows from the definition of the slipline field of the models. When crossing the border given by relation $(2.9 \mathrm{~b})$, the slipline field comes out of the flat surface and lies on top of it, as can be seen as a difference between figure 2.9a and 2.9b, which makes the difference between only plastic deformation of the material and at the wedging site in wearing off of material. Relation (2.9a) is not defined by the models given in [10]. The slipline models do not cover the whole wear mode diagram; for some combinations of 
$f_{H K}$ and $\theta$ no model is available. For example, the cutting model is only valid if $\theta$ exceeds 45 degrees, according to real practice, cutting may already occur in the case that $\theta$ has much lower values. Other models, for example Challen and Oxley [11], show that a cutting model can also be used in cases for lower values of $\theta$. For the transition given by relation (2.9a) in [39] no basis is given. In [11] a relation for the transition is given between the cutting regime and the others. This relation is dependent on the work hardening of the plastic material. In the case of no work hardening, this relation reads:

$$
\theta=\frac{\pi}{2}+\frac{1}{2} \arcsin \left[f_{H K} \sin \left\{2 \arctan \left(1+\frac{\pi}{2}\right)\right\}\right]-\arctan \left(1+\frac{\pi}{2}\right)
$$

which is near the transition given in relation (2.9a) as can be seen in figure 2.10. Because the transition given by equation (2.9a) is close to the one given by equation (2.10), only relation $(2.9 \mathrm{a})$ will be used further on.

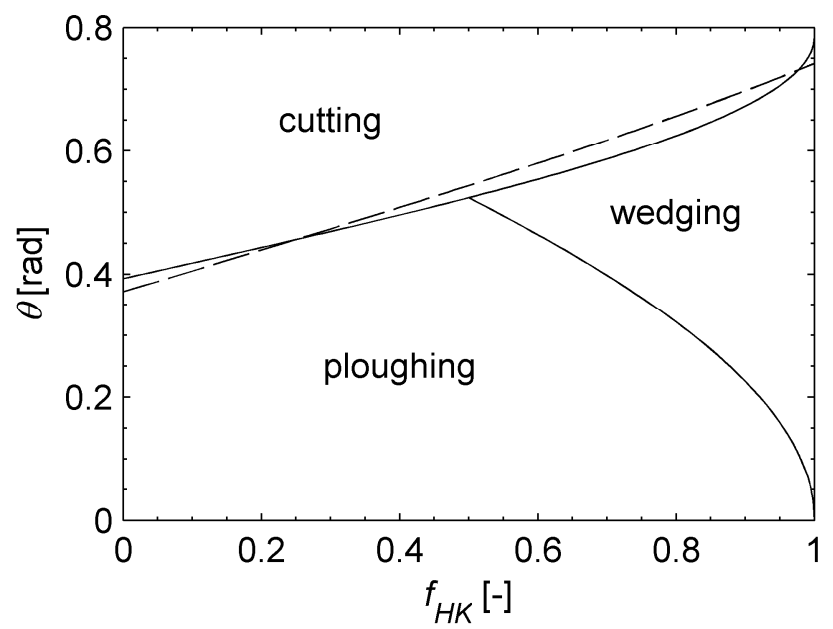

Figure 2.10. Domains of the different wear regimes with solid lines after [39] and dashed line after [11].

\subsection{Existing galling models}

In the following sections (2.3.1 and 2.3.2) two models will be shown which each deal with a certain stage in the galling phenomenon. The model shown in section 2.3.1 is a model for lubricated deep drawing contacts and predicts when a lubricant layer fails. When the lubricant fails, this enables direct contact between tool and sheet, so, a possibility for 
material transfer is born. The model given in section 2.3.2 is a lump growth model, which predicts the lump growth in the case of unlubricated contacts.

The models model the contact in the manner as discussed in section 2.2. A purely plastic contact is assumed. The sheet material is relatively soft compared to the tool material, so the sheet will deform in a plastic manner. Because the sheet is rougher than the tool, plateaus will be formed on the sheet, which have contact with the tool. The asperities of the tool, in their turn, scratch into these plateaus. During this scratching the soft sheet material may transfer to the tool asperities. The models focus on the contact between these tool asperities and tool plateaus. The latter is for sake of simplicity assumed to be flat. Relation (2.6) can be used to determine the separation of the two surfaces, which summits are in contact and the attack angle of each summit that is in contact.

\subsubsection{Initiation model}

To initiate galling in a forming process, some conditions have to be satisfied. One of the conditions is direct contact between tool and workpiece material. Van der Heide [20], [21], [22] uses this condition in the case of lubricated deep drawing of sheet metal. The hypothesis in this work is: "Galling initiation in lubricated sheet metal forming processes occurs at asperity level as a result of the fact that the lubricant's critical temperature is exceeded, due to frictional heating". Based on this hypothesis a model is formulated. The model focuses on asperity level. The asperities which are in contact are determined. In these asperity contacts the flash temperature is calculated as a balance between heat generated due to friction and carried away by conduction of the sheet and tool material and convection of the sliding sheet.

In [20] the asperities are assumed to be ellipsoid shaped. The contact spots have an elliptical shape with dimensions $a$ and $b$, respectively the radii in sliding direction and perpendicular to it.

The generated heat $q$ in the contact spots is determined as follows:

$$
q=f \cdot F_{n} \cdot v
$$

In this relation $f$ is the coefficient of friction, $F_{n}$ the normal force on the asperity and $v$ the velocity difference between the two contact surfaces. $F_{n}$ will be calculated as given in equation (2.8) multiplied by 0.5 , because only the frontal half of the asperity is in contact. The friction coefficient $f$ is calculated by the relations given by Challen and Oxley [10]. These relations are given below, where the subscripts $p l, w$ and $c$ respectively point to the ploughing, wedging and cutting regime:

$$
f_{p l}=\frac{\xi_{1} \sin \theta+\cos \left(\arccos f_{H K}-\theta\right)}{\xi_{1} \cos \theta+\sin \left(\arccos f_{H K}-\theta\right)}
$$




$$
\begin{gathered}
f_{w}=\frac{\left(1-2 \sin \xi_{2}+\sqrt{1-f_{H K}^{2}}\right) \sin \theta+f_{H K} \cos \theta}{\left(1-2 \sin \xi_{2}+\sqrt{1-f_{H K}^{2}}\right) \cos \theta-f_{H K} \sin \theta} \\
f_{c}=\tan \left(\theta-\frac{\pi}{4}+\frac{1}{2} \arccos f_{H K}\right)
\end{gathered}
$$

with:

$$
\begin{gathered}
\xi_{1}=1+\frac{\pi}{2}+\arccos f_{H K}-2 \theta-2 \arcsin \left(\frac{\sin \theta}{\sqrt{1-f_{H K}}}\right) \\
\xi_{2}=\theta-\frac{\pi}{4}-\frac{1}{2} \arccos f_{H K}+\arcsin \left(\frac{\sin \theta}{\sqrt{1-f_{H K}}}\right)
\end{gathered}
$$

The flash temperature $\Theta$, in fact temperature rise, is proportional to the generated heat and reciprocal with the thermal conductivity $K$. So:

$$
\Theta \sim q / K
$$

To calculate the flash temperature, a relation of Bos [4], [5] is used in which the conduction and convection of heat is combined. The conditions of deep drawing are assumed. The asperities of the tool have no velocity, so are continuously in contact. So, the tool is only able to transport heat out of the contact by conduction. The sheet material slides over the tool. Besides the conduction, this sliding contributes to the convection of heat out of the contact. For these conditions the following relation is found:

$$
\Theta=\frac{f \cdot F_{n} \cdot v}{\sqrt{a b}}\left(\frac{1}{\frac{K_{\text {tool }}}{0.375 \cdot \Lambda}+\frac{K_{\text {sheet }}}{\theta_{\text {sheet }}}}\right)
$$

The subscripts tool and sheet refer to each body in contact. $\theta_{\text {sheet }}$ is defined as:

$$
\theta_{\text {sheet }}=\left[(0.375 \cdot \Lambda)^{\lambda}+\left(\frac{0.589}{\sqrt{\varphi \cdot \frac{a \cdot v}{\kappa_{\text {sheet }}}}}\right)^{\lambda}\right]^{1 / \lambda}
$$

In which $\kappa$ is the thermal diffusivity of the sheet material, defined as $K / \rho c_{p}$. Further: 


$$
\begin{gathered}
\varphi=b / a \\
\lambda(\varphi)=0.5 \exp (1-\varphi)-2.5 \\
\Lambda(\varphi)=\frac{2 \sqrt{\varphi}}{1+\varphi} \cdot \frac{2}{\pi} \cdot \mathrm{K}\left(\frac{|1-\varphi|}{1+\varphi}\right)
\end{gathered}
$$

In the last relation $\mathrm{K}(n)$ is the complete elliptic integral of the first kind with modulus $n$.

For every asperity contact the flash temperature can be calculated using equations (2.14) to (2.18). If the lubricant's critical temperature is exceeded, a condition is satisfied to cause galling. The next condition which is used in [20] is that an asperity contact is in the wedge regime, an assumption which is used in the growth model of De Rooij [39], a model which is described in section 2.3.2.

\subsubsection{Lump growth model}

Lump growth in deep drawing contacts is modelled by De Rooij [39], [40]. The proposed model describes the lump growth on asperity level. The model is based on the wear mode diagram of Challen and Oxley [10], see section 2.2.

In [39] it is assumed that material transfer will not occur on all contacting summits, but only on summits which are in the wedge regime. In the ploughing regime no material removal by the sliding tool asperity occurs, so no lump growth on the tool will occur. The other regimes produce wear debris, which is, in principle, available for material transfer. However, wear debris will most likely be transferred to the low parts of the tool surface and not to the most critical spots, the asperities. Besides this, transferred material will not be very strongly fixed to the tool surface, because of oxidation layers and other factors inhibiting adhesion. For this reason in [39] the wedge-formation is taken into account, because in this regime a strong adhesion may result from the generation of virginal, unprotected contact surface during wedge-formation.

It is assumed that only a fraction $m$ of the material that wears off from the sheet in the wedge regime attaches to the tool summits. From this assumption the following relation for the lump growth (height increase) $\Delta s$ is derived:

$$
\Delta s=m \cdot \frac{V_{\text {wear }}}{A_{\text {summit }}}
$$

In equation (2.19) $V_{\text {wear }}$ is the volume of the material that wears off in the wedge regime and $A_{\text {summit }}$ gives the surface area of the summits on which the material of $V_{\text {wear }}$ is deposited. The fraction $m$ is assumed to be proportional to the adhesion force $F_{a}$ :

$$
m \sim F_{a}
$$


Different types of adhesion regimes exist. In this model two regimes are taken into account, which results in:

$$
F_{a}= \begin{cases}2 \pi H \beta\left(s-h_{s}\right) & \text { if } H^{2}<\frac{2 \Delta \gamma E^{*}}{\pi \sqrt{2 \beta\left(s-h_{s}\right)}} \\ \frac{2 \Delta \gamma E^{*}}{H} \sqrt{2 \beta\left(s-h_{s}\right)} & \text { if } H^{2}>\frac{2 \Delta \gamma E^{*}}{\pi \sqrt{2 \beta\left(s-h_{s}\right)}}\end{cases}
$$

In this relation $s$ is the summit height, $h_{s}$ the nominal surface height, $\beta$ the summit radius, $\Delta \gamma$ the specific adhesion energy and $E^{*}$ the reduced modulus of elasticity.

The wear volume in the wedge regime derived from the wear rate given by Challen and Oxley [10] gives the following:

$$
V_{\text {wear }}=\frac{1}{2 k} \frac{\sin ^{2} \theta+\frac{1}{2} \sin 2 \theta}{1+\sin 2 \theta} F_{n} l_{\text {slide }}
$$

with:

$$
k=\frac{H}{3 \sqrt{3}}
$$

In this relation is $\theta$ the attack angle, $k$ the shear strength of the wearing material and $l_{\text {slide }}$ a certain sliding distance.

It is assumed that due to material transfer the asperity grows in such a manner that $\beta$ remains constant. This is implemented as a semi-spherical asperity lift-up with a value $\Delta s$ from the surface, which results in a summit area of:

$$
A_{\text {summit }}=\pi \beta^{2}
$$

Using relations (2.22a) and (2.23) in (2.19) gives:

$$
\Delta s=m_{2} F_{a} 3 \sqrt{3} \cdot \frac{\sin ^{2} \theta+\frac{1}{2} \sin 2 \theta}{1+\sin 2 \theta} \cdot \frac{s-h_{s}}{\beta}
$$

The fraction $m$ as given in equation (2.19) is replaced by $m_{2} \cdot F_{a} / l_{\text {slide }}$ in equation (2.24). According to equation (2.20), $m$ is proportional to $F_{a}$. Assuming that $m$ is only a function of $F_{a}, m_{2} / l_{\text {slide }}$ becomes a constant. For a product where the sliding length already is determined by the sliding length, $m_{2}$ becomes a constant as well.

Relation (2.24) has to be calculated for every summit in the wear regime. In this way, an incremental change over the whole surface area of interest can be calculated. The next step is to repeat this action a number of time steps, to get the development of the lump growth in time. In [39] this development is presented as the change of the summit height density 
function. Likewise, the model can be implemented for a set of summits, instead of a summit height density function. In the case of a set of summits it can be combined with the initiation model of section 2.3.1. In that situation, both the effect of critical temperature on the lubricant and the lump growth itself will be taken into account.

\subsection{Summary}

The contact between workpiece and tool is discussed on the different length scales, that is on macro, meso and micro scale, in particular the effect of roughness. The contact stresses as well as deformations on each scale were analysed. With respect to galling, models are investigated that concern the initiation of galling and the lump growth.

The objective of this research is the development of a Galling Performance Indicator, as depicted in chapter 1. For this development the lump growth model of De Rooij will be extended. The model of De Rooij has only a one dimensional lump growth, namely, growth in height, as given in equation (2.24). In chapter 3 experiments will be presented that show not only lump growth in height, but also in frontal direction. This effect will be modelled in chapter 4 for the single asperity case. 


\section{Material transfer on a single asperity scale: experiments}

In this chapter experimental work will be presented which is used for studying the behaviour of a hard metal asperity that scratches into a softer material. In a deep drawing process, this represents on micro scale the situation in which material transfer from the soft workpiece material to the hard tool asperity may take place. The development of a dedicated setup and the experiments will be discussed in this chapter.

In chapter 2 different scales have been discussed. For determining the tribological properties and finally the determination of the galling sensitivity, all the scales have their own role. The larger scale creates the environment and the input values for the smaller scale. The macro scale is the scale with values related to deep drawing FEM simulations, the meso scale requires information from the macro scale and from the roughness texture as well as the material properties. The micro scale is the scale where galling actually takes place. At that scale, the material adheres to the counter surface. Therefore, the micro scale is the scale of interest for experiments. In the experiments, a single asperity of tool material scratches on sheet material. The results obtained on the micro scale will be used to determine the galling performance on the meso scale, because on this scale the interactions of all the asperities in a certain area can be taken into account. The results on the meso scale can be mapped on the macro scale, which results in a galling risk map for a product. In this chapter the experimental setup will be described in section 3.1, the used samples and their properties in section 3.2, the actual experiments with their results in section 3.3. This chapter will end with the conclusions from the experiments in section 3.4.

\subsection{Experimental setup}

\subsubsection{Ploughing Asperity Tester}

To study the contact in a deep drawing process at a single asperity scale, a dedicated tester has been developed. In the following, this tester will be called Ploughing Asperity Tester. The main aspects of the tester will be discussed below.

In deep drawing the two contact bodies have different properties. The tool surface is a hard surface which comes into contact with the workpiece during every production cycle. The sheet surface has a lower hardness than the tool. The sheet only comes into contact with the tool for the duration of one production cycle. The Ploughing Asperity Tester is depicted in figure 3.1a. The tester is of the type pin-on-flat and has some special properties. Some characteristics of the device are: 
- The scratch tracks are in a straight line.

- The load of the pin can be controlled actively.

- $\quad$ It is possible to make a number of scratches next to each other, see figure $3.1 \mathrm{~b}$. These characteristics give the tester some important properties. The straight line scratch tracks generate scratches of a restricted length $l$. The total scratch length of a scratch pin can be extended by repeating this length $n$ times. This gives the possibility of always having fresh contact material on the flat contact body, by doing every track next to each other, instead of in the same track.

In the experiments the tool is represented by a scratch pin of tool steel, sample $A$ in figures 3.1 and 3.2. For sample $B$, a piece of sheet metal is used. Sample $A$ has a spherical shaped tip with a radius in the order of a single roughness asperity of the tool. Sample $A$ makes straight tracks side by side on sample $B$, in order to have always a fresh sheet metal surface in the contact, as in deep drawing, where a sheet only makes contact during one production cycle. This makes the Ploughing Asperity Tester much more close to the actual situation on micro scale of a deep drawing process than a standard pin-on-disk tester.

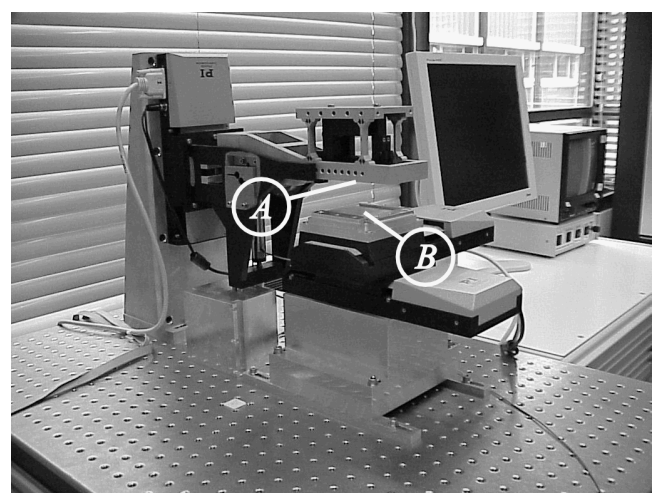

$a$

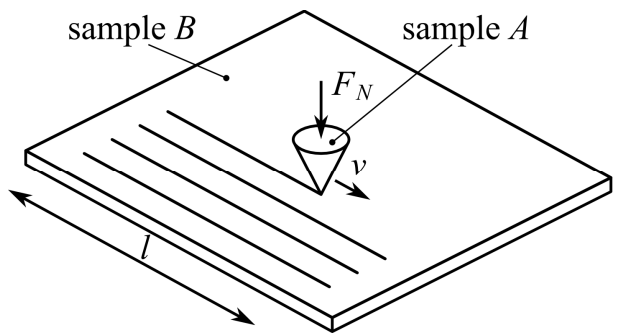

$b$

Figure 3.1. Ploughing Asperity Tester. A photo of the device (a) and a schematic view of the scratch tracks (b).

To fulfil its tasks, the Ploughing Asperity Tester is equipped with actuators and measurement sensors. A schematic view of the device is given in figure 3.2. To move the samples relative to each other, the flat sample $B$ is placed on a $x y$-table. This table is built by perpendicular stacking of two linear stages. The scratch sample $A$ is mounted on the beam, that is driven by two actuators in series. The coarse displacement in $z$-direction is done by a linear stage (as used in the $x y$-table), this is shown in figure 3.2a. The small displacements in $z$-direction are done by a piezo, see figure $3.2 \mathrm{~b}$. 


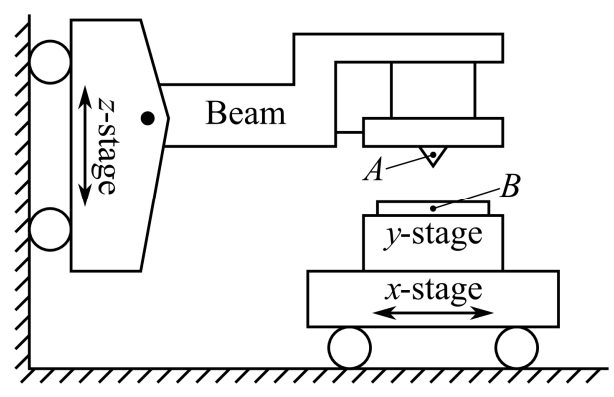

$a$

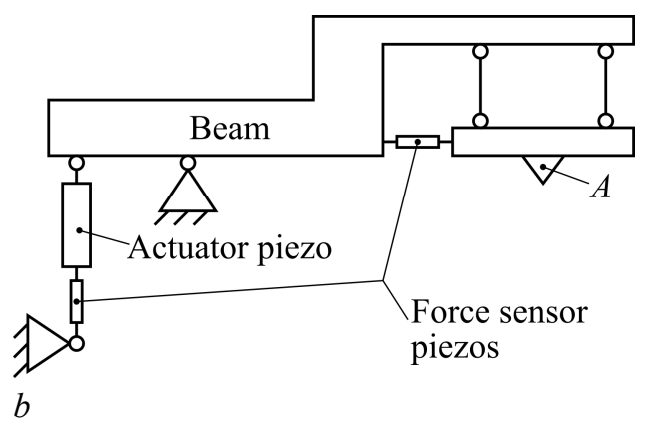

Figure 3.2. Schematic views of Ploughing Asperity Tester. The large displacements are done by linear stages (a). A detail of the beam with the actuator piezo for small vertical displacements of the scratch sample and the measurement piezos for the normal and the friction force (b). ' $A$ ' is the scratch sample, ' $B$ ' the flat sample.

Some basic specifications are given in table 3.1. Some remarks will be made on these values:

- The area that can be used for fresh contact on the flat sample, is the track length times the track area width. In the case of scratch track width in the order of 0.1 $\mathrm{mm}$, a distance between the tools $\Delta y$ of $1 \mathrm{~mm}$ after every scratch in $x$-direction is a reasonable distance, considering effects such as shoulder formation. In this manner a total scratch length of $50 \times 50 \mathrm{~mm}=2.5 \mathrm{~m}$ can be made on a sample of $70 \times 70 \mathrm{~mm}$.

- The maximum normal force of $50 \mathrm{~N}$ is the absolute maximum including the fluctuations on the signal. That means that the practical maximum nominal normal force is about 30 or $40 \mathrm{~N}$, depending on the measurement conditions.

- The velocity given of $50 \mathrm{~mm} / \mathrm{s}$ is the maximum possible velocity of the device. To get a more stable force controlled contact situation, a velocity in the order of 1 $\mathrm{mm} / \mathrm{s}$ or lower has to be chosen.

\begin{tabular}{l|l} 
Property & Maximum value \\
\hline Track length & $50 \mathrm{~mm}$ \\
Number of tracks & 50 \\
Normal contact load & $50 \mathrm{~N}$ \\
Scratch velocity & $50 \mathrm{~mm} / \mathrm{s}$
\end{tabular}

Table 3.1. Specifications of Ploughing Asperity Tester. 


\subsubsection{Load control and contacts}

For performing the experiments, the Ploughing Asperity Tester control software and samples with sample holders have been developed. The developed software fulfils the following tasks: applying a normal force controlled contact between the sample that represents a single asperity of the tool and the sheet material, steering the scratch movement of one sample over the other and saving the measured normal and tangential forces. The sample holders are developed for holding the single asperity sample and the piece of sheet metal.

\section{Control software}

To improve the usability of the Ploughing Asperity Tester, software is developed to control the tester. The tester is an in-house developed device, which is assembled from, among other things, a number of actuators and sensors that act as separate hardware components. These components each have their own peripheral equipment that communicates with a computer. The control software is created in LabView, a programming environment for creating virtual instruments. In LabView, the focus is on the design of the user interface and on the communication with and control of lab instruments. Two types of functionality of the tester will be discussed in more detail below, respectively the force controlled loading of the samples and the parallel track functionality.

Displacement controlled experiments demand very flat samples. Further, any tilt in the sample has to be avoided. Any waviness or tilt results in a fluctuating normal force during scratching due to deformation of the tester, see figure 3.3a. Polished samples are often really flat. Sheet metal is not flat enough to get a (more or less) constant normal force or indentation depth during scratching. A fluctuating indentation depth has a strong influence on the attack angle. The scratch pins that have been used have a tip radius. Using the dimensions as given in figure 2.7 the following derivative can be found, with $\theta$ in radians:

$$
\frac{d \theta}{d \delta}=\frac{1}{\beta} \frac{1}{\sqrt{\frac{\delta}{\beta}\left(2-\frac{\delta}{\beta}\right)}}
$$

Using equation (3.1) the effect of displacement controlled experiments on not-flat samples can be investigated. In most experiments a tip radius of $\beta=50 \mu \mathrm{m}$ is used. For $\theta=45^{\circ}$, that means $\delta=15 \mu \mathrm{m}, d \theta / d \delta=1.6^{\circ} \mu \mathrm{m}^{-1}$. This derivative increases for decreasing values of $\delta$ and goes to infinite for $\delta=0$. The effect of a not-flat sheet is compensated a bit by the stiffness of the tester, which is in $z$-direction at the contact spot of the samples about $10^{2} \mathrm{~N}$ $\mathrm{mm}^{-1}$. Normal forces are in the order of $1 \mathrm{~N}$, so the effect of stiffness, or better, the flexibility effects, is insignificant. In practice, over a track length of $50 \mathrm{~mm}$, a flatness 
tolerance for a sheet metal surface (for tilt and waviness) within $10 \mu \mathrm{m}$ is hard to realize, so force feedback is necessary to apply a constant contact load. The developed control software is able to communicate with all the actuators and sensors of the tester. This property is used to give force feedback to the actuators that bring the samples in contact. In this manner a closed loop is created between the measured normal force and the actuators acting in normal direction. In figure $3.3 \mathrm{~b}$ the action of the normal actuators is shown by the dashed line and its deformation consequences on the tester. In figure 3.3c the result of a measurement is given that shows a constant normal load (regarding the low frequency component) due to the force feedback control.

For simulating the deep drawing contact, a fresh sheet surface is demanded. This is realized by the parallel track function, which moves the $y$-stage a small distance $\Delta y$ after every scratch made in $x$-direction.

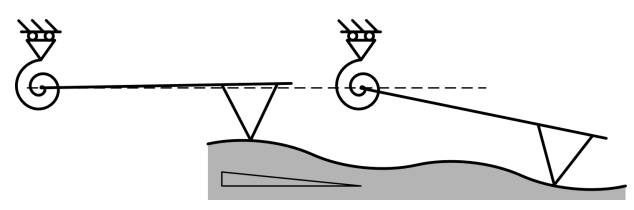

$a$

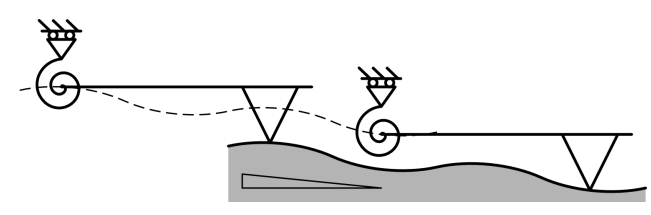

$b$

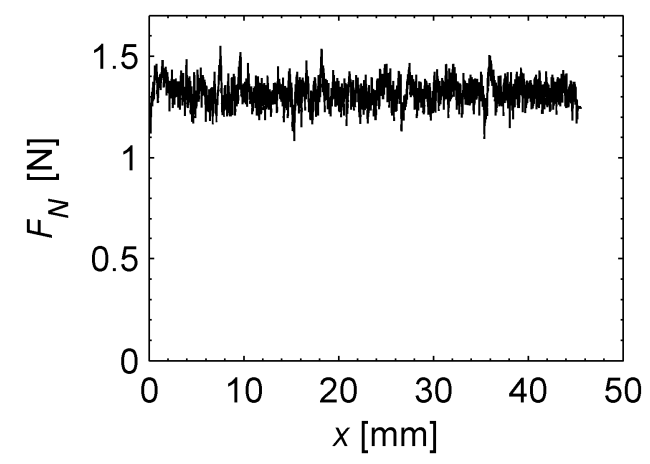

$c$

Figure 3.3. Displacement (a) and force controlled (b) scratching on a tilted and wavy surface and its deformation consequences. The beam on which the scratch sample is mounted has a stiffness, here represented as a torsion spring. The dashed line describes the track of the rotation point of the beam. Applying force control results in a constant normal force as given in the example measurement (c). 


\section{Samples and sample holders}

For the Ploughing Asperity Tester both contact samples have been developed and for the lower contact body, the piece of sheet metal, also the sample holder.

The scratch pin is made of tool material WN1.2379. The tip geometry and the material of the pin are prescribed, the rest can be adapted for a standard holder. The sample is designed for using a holder that is suitable for samples with a shaft of $6 \mathrm{~mm}$ diameter. The sample is given in figure $3.4 \mathrm{a}$. Details about the material and the tip geometry are given in section 3.2 .

The lower contact body in the experiments is a piece of sheet metal. A special holder for this sample is designed. The bottom side has to be mounted on a linear stage, the $y$-stage as given in figure 3.2a. The mounting possibilities are determined by the hole pattern on the linear stage. At the top side of the holder a piece of sheet metal has to be clamped. A sample size of $70 \times 70 \mathrm{~mm}$ is chosen. This gives the possibility to use the full stroke lengths of the linear stages $(50 \mathrm{~mm})$ and a margin of $10 \mathrm{~mm}$ remains around the measurement area that can be used to clamp the sample. The sample holder is shown in figure $3.4 \mathrm{~b}$.
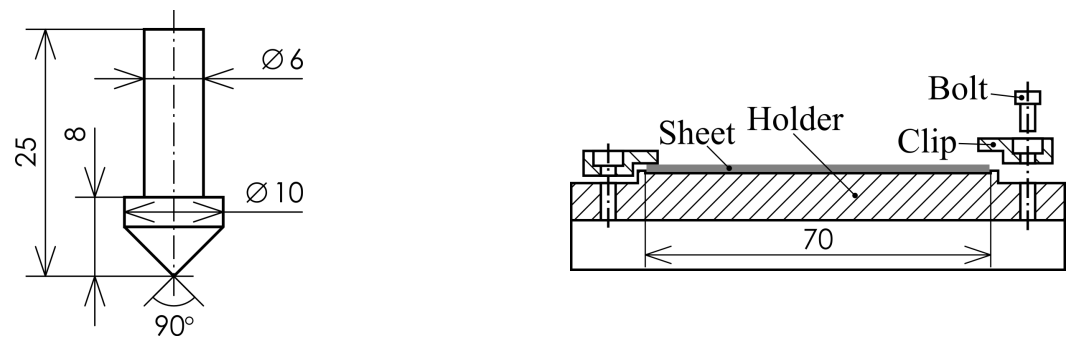

$a$

$b$

Figure 3.4. Dimensions of scratch pin (a). At the conical tip a radius is polished with a prescribed radius that represents an asperity. The holder for the sheet material with its clamping system (b).

\subsection{Samples used in the experiments}

The purpose of the experiments is to study the interaction of a tool asperity in contact with sheet metal. The experiments are performed with the Ploughing Asperity Tester as described in section 3.1. The samples and contact conditions are chosen in such a manner, that the contact agrees in many respects with the contact in the deep drawing process. Further details of the parts used in the experiments are given in the following sections. 


\subsubsection{Tool asperity}

The tool asperity is represented by the scratch pin in the Ploughing Asperity Tester. This sample is labelled as $A$ in figures 3.1 and 3.2 and its shape is given in figure $3.4 \mathrm{a}$. In the figure only the macro geometry is given. The essential part of the pin is the tip of the cone. To approach the contact of a single tool asperity during deep drawing, the material and the geometry are chosen close to a tool asperity.

After the pin is made and hardened, the conical area of the hardened pin is finished with a spherical tip by grinding and polishing. The pins are polished in two batches. One batch has a tip radius of $50 \mu \mathrm{m}$, the other of $100 \mu \mathrm{m}$.

\subsubsection{Sheet material}

The focus within this research is on one type of aluminium sheet and zinc coated steel sheet. These materials are used in the experiments. The aluminium sheet is AA5182 EDT and the zinc coated steel sheet is DX54D Z EDT. The materials have the same type of surface texture, given by the abbreviation EDT (electrical discharge texturing). This is an isotropic texture and is chosen to avoid orientation effects of the texture.

The materials are commercially available. No modification of the material is applied. From a piece of sheet metal a sample is cut of $70 \times 70 \mathrm{~mm}$, to fit into the holder shown in figure $3.4 \mathrm{~b}$. The sheet material may contain a layer of conservation oil, which is why a sample is always degreased before an experiment. In the case of lubricated experiments, after the degreasing step the lubricant is applied to the surface. Details about degreasing and applying lubricant are given in section 3.2.3.

Below, data will be given of the sheet materials that are used in the experiments. In table 3.2 properties are given of DX54D Z EDT. SEM pictures of the surface texture and a cross section of zinc layer are given in figure 3.5.

\begin{tabular}{l|l} 
Property & Value \\
\hline$R_{q}$ & $1.0 \mu \mathrm{m}$ \\
Bulk hardness & $81 \mathrm{HV}_{0.2}$ \\
Zinc layer thickness & $14 \mu \mathrm{m}$ \\
Zinc layer hardness & $46-57 \mathrm{HV}_{0.005}$
\end{tabular}

Table 3.2. Properties of DX54D Z EDT. 


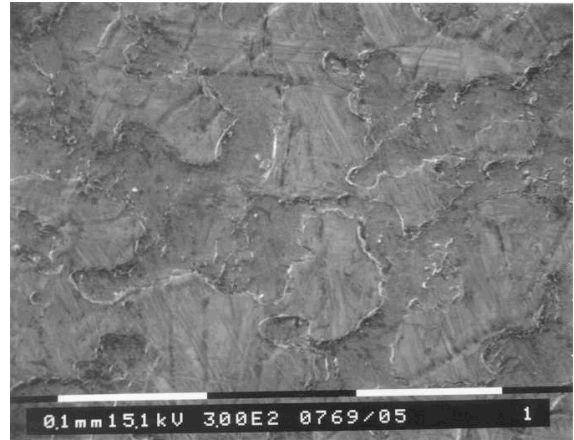

$a$

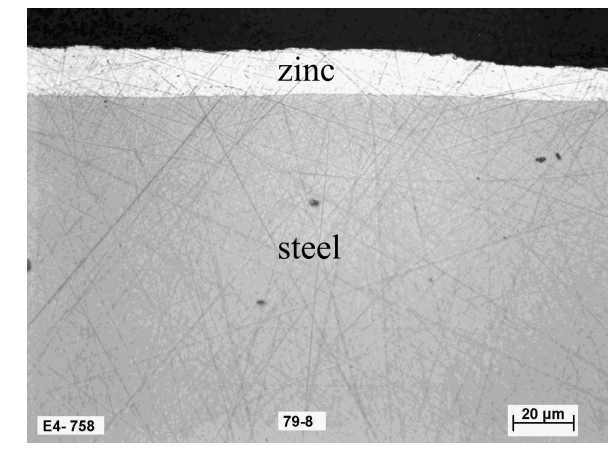

$b$

Figure 3.5. SEM pictures of DX54 Z EDT sheet material. The surface texture top view (a) and a cross section of the sheet material near the zinc layer (b).

In table 3.3 properties are given of AA5182 EDT and in figure 3.6 a SEM picture is given of its surface texture.

\begin{tabular}{l|l} 
Property & Value \\
\hline$R_{q}$ & $1.4 \mu \mathrm{m}$ \\
Bulk hardness & $68 \mathrm{HV}_{0.2}$
\end{tabular}

Table 3.3. Properties of AA5182 EDT.

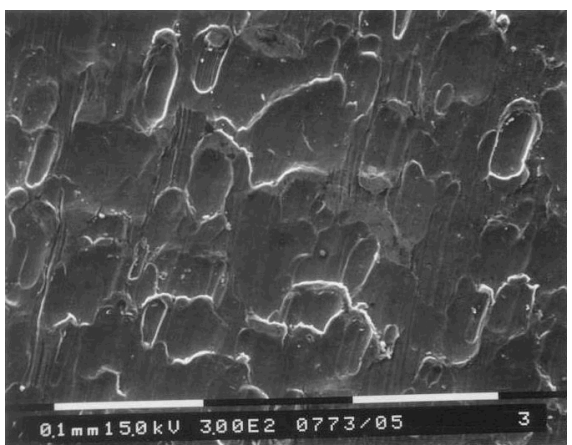

Figure 3.6. SEM picture of the surface texture of AA5182 EDT. 


\subsubsection{Lubricant}

A part of the experiments has been performed with a lubricant on the sheet material. In sheet metal forming processes the main function of the lubricant is to control the friction in the sliding contact between the sheet and the tools. Another function of the lubricant is to avoid or reduce galling by preventing or reducing direct contact between the sheet metal and the tools. Only one type of lubricant has been used, namely Quaker N 6130. Quaker N 6130 is a deep drawing lubricant with rust preventive functionality.

Before performing the experiments the samples, both the scratch pin and the sheet material, are degreased. The samples are degreased using acetone and subsequently dried with a tissue. Next, an oil film is applied on one side of the sheet sample. The sample is drained by holding it in a vertical orientation for some hours. The amount of oil is determined by the difference of the mass before and after applying the lubricant. In this manner a typical amount of lubricant is about $5 \mathrm{~g} / \mathrm{m}^{2}$.

\subsection{Experiments}

\subsubsection{Experimental strategy}

To study the galling behaviour a number of experiments have been performed. To get insight in the material transfer behaviour a number of parameters have been varied. Most parameters are already discussed in section 3.2, namely, the tip radius, the sheet material and whether the contact is lubricated or not. Further, the scratch length and the normal force have been varied. The normal force causes the penetration of the scratch pin in the sheet material and so the change of the attack angle. The sliding velocity is kept constant on 1 $\mathrm{mm} / \mathrm{s}$ in all experiments.

First, results of the lubricated experiments will be shown. These results are used to investigate the effect of lubrication and the agreement with the initiation model as given in section 2.3.1. Next, the lump growth is investigated in more detail under degreased conditions.

The range of normal forces that is applied to the scratch pin is determined in the following manner. According to the models given in the sections 2.3.1 and 2.3.2, galling happens in the wedge regime. The possible attack angles of this regime are in the range of 0 to $45^{\circ}$. The maximum possible attack angle that can be caused by the scratch pin is $45^{\circ}$, this from a geometrical point of view, so experiments can be performed on every point in the wedge regime. The normal force needed to create a certain attack angle will be determined on the basis of the geometry of the tip and the hardness of the sheet material, using equation (2.5). In the last part of equation (2.5) a summit is assumed to be a paraboloid. In the case of a spherical shape, the following relation is found for the normal force: 


$$
F_{n}=0.5 \pi a^{2} H=0.5 \pi H \beta^{2} \sin ^{2} \theta
$$

In equation (3.2) the correction factor 0.5 for the fully plastic behaviour is used, because during scratching of the pin, only the frontal part of it is in contact.

\title{
3.3.2 Lubricated experiments
}

A number of lubricated experiments are performed. The type of lubricant and the method to apply the lubricant to the sheet material is discussed in section 3.2.3. The experiments are performed on zinc coated steel sheet and on aluminium sheet material.

\begin{abstract}
Aluminium sheet
Experiments are performed using a conical scratch pin with a tip radius of $50 \mu \mathrm{m}$. In the different experiments, the normal force is varied. The range of normal forces is from $0.2 \mathrm{~N}$ to $2.5 \mathrm{~N}$. The corresponding attack angles can be found by using equation (3.2). The hardness of the material is given in table 3.3. A hardness given in MPa can be approximated by multiplying the Vickers hardness with 10 . This results in a hardness of $680 \mathrm{MPa}$. Then the range of attack angles becomes 16 to $45^{\circ}$. For forces above $1.3 \mathrm{~N}$, the spherical part of the scratch pin is fully penetrated into the sheet material, so the conical part comes in contact with the sheet, resulting in the attack angle of $45^{\circ}$. For this series of experiments the scratch track is $20 \mathrm{~mm}$.

After the experiments the pins are investigated for material transfer. For all values of the normal force it has been observed that material was transferred to the scratch pin. The adherence of the transferred material to the pin was not strong enough to survive an ultrasonic cleaning treatment. Only once did a layer of about $1 \mu \mathrm{m}$ remain on the pin after an ultrasonic cleaning treatment and cleaning it with a tissue. An image of the pin, made by an optical microscope, is shown in figure 3.7. No direct relation is found as a function of the normal force and the strength of the transferred aluminium layer.
\end{abstract}




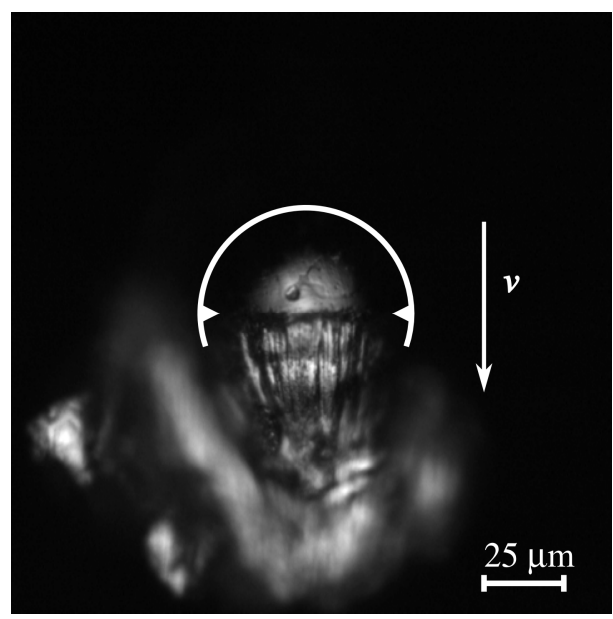

$a$

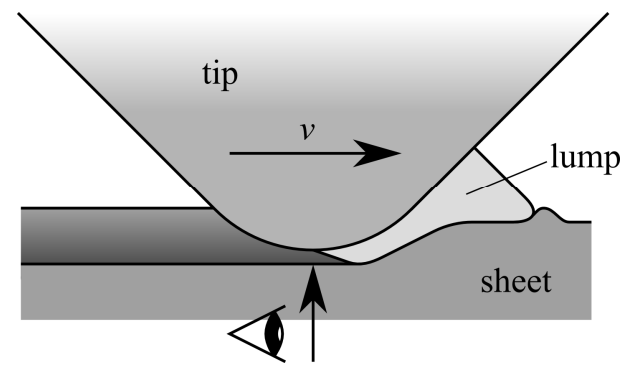

$b$

Figure 3.7. A scratch pin that ploughed through lubricated AA5182 EDT sheet material (a). The scratch length is $20 \mathrm{~mm}$, the normal force $2 \mathrm{~N}$. The pin is already ultrasonic cleaned, but still not cleaned by hand with a tissue. In spite of the lubrication, aluminium is transferred to the pin and this transfer layer survived the ultrasonic cleaning. In this figure the pin is positioned concentric with the circle segment. The part of the surface above the two spikes on the circle segment is the original pin surface that was not in contact. Below the spikes a 'rough surface' is visible, which is the transferred material. The image is only partly in focus, due to the small depth of field of the used optical microscope. The grey parts near the bottom of the image that are out of focus show transferred aluminium. The orientation of the microscope with respect to the pin is shown by the arrow with eye symbol (b), which also shows how the pin is oriented during the experiment. The scratch direction of the pin is given by the arrow of the velocity $v$.

\section{Zinc coated steel sheet}

Another set of experiments has been performed with zinc coated steel sheet. The scratch pin is of the same type as given before for experiments with aluminium sheet and the same range of normal forces is used. The hardness of the zinc layer as given in table 3.2 corresponds to about $500 \mathrm{MPa}$. The range of attack angles becomes, using equation (3.2), 16 to $45^{\circ}$, where $45^{\circ}$ is reached for a normal force of $0.98 \mathrm{~N}$. So, for the normal force range where the spherical part of the scratch pin is not fully penetrated in the sheet material, the attack angle has a higher value for a given normal load, compared to the case of aluminium sheet, because of the lower hardness of the zinc layer. This calculation of the attack angle is 
only true for small penetrations where only the hardness of the zinc layer is of importance. For larger penetrations, in the order of the zinc layer thickness, the hardness of the steel substrate also has to be taken into account. Because of the small differences in results of the experiments, a better estimation of the attack angle taking the layered structure into account will be omitted.

On the scratch pins used in the experiments with lubricated zinc coated steel sheet no transferred material can be observed. Only wear particles of the zinc layer can be found near the scratch track for the experiments with normal forces of $1 \mathrm{~N}$ and above. The contacts with these applied normal forces are in the cutting regime, which explains the formation of these wear particles. Images of some pins that are used in these experiments are shown in figure 3.8. After almost every measurement the microscope images look like figure 3.8a. An exception is shown in figure $3.8 \mathrm{~b}$, but after degreasing this pin also looks like figure $3.8 \mathrm{a}$. In the case of figure $3.8 \mathrm{~b}$, there is only a weak adhesion due to the lubricant that adheres the wear particles to the pin.

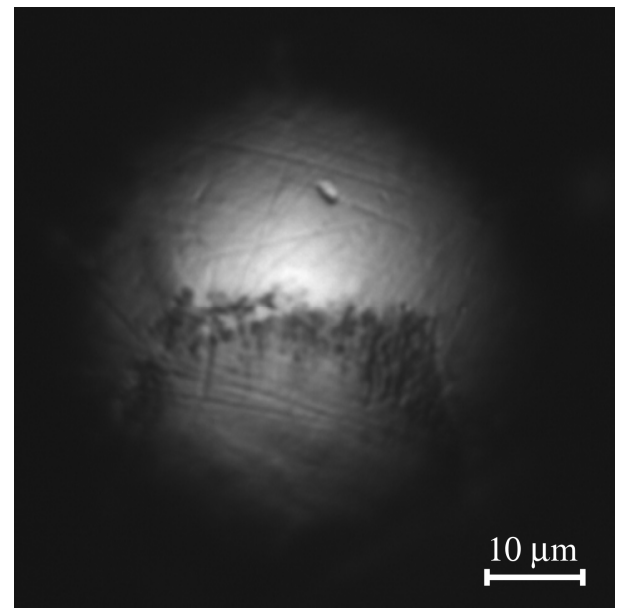

$a$

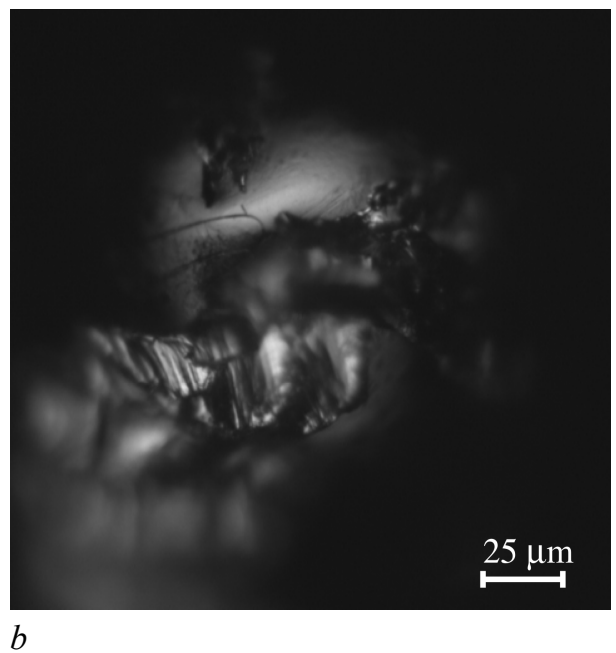

b

Figure 3.8. Scratch pins that ploughed through lubricated DX54D EDT sheet material. In one situation no material is transferred and the colour is slightly changed only at the contact spot (a). In another situation, zinc particles are visible (b), but after degreasing the pin, the wear material is removed. The orientations of sliding direction of the pins and the camera position relative to the pins are as in figure 3.7. 


\section{Discussion of the lubricated experiments}

From the lubricated experiments that are performed, a big difference can be observed in the material transfer behaviour between aluminium and zinc coated sheet material. In the case of zinc coated sheet material, in a number of experiments wear particles are found, but no material transfer takes place. In the case of the aluminium sheet material transfer can be observed.

In the case of lubricated contacts, the flash temperature in the contact can play a role as is presented in section 2.3.1. An estimation of the flash temperature can be made using equation (2.14). To make this estimation, equation (2.14) will be adapted. The coefficient of friction times the normal force can immediately be replaced by the (measured) friction force. The scratch pin has a conical shape, so the assumption that the contact bodies are half spaces is not justifiable. Therefore, for the first estimation, the conduction to the scratch pin will be omitted. Further, for simplicity the convection part of the sheet material will be omitted. These adaptations result in the following simplified relation for the flash temperature:

$$
T_{f}=\frac{0.375 F_{w} \cdot v \cdot \Lambda}{\sqrt{a b} K_{\text {sheet }}}
$$

The thermal conductivity of aluminium AA5182 is $126 \mathrm{~W} \mathrm{~m}^{-1} \mathrm{~K}^{-1}$. The maximum value of $F_{w}$ in the experiments is $3 \mathrm{~N}$ and the minimal values of the radii $a$ and $b$ are 7 and $14 \mu \mathrm{m}$. The tip of the scratch pin has a spherical shape and therefore in each direction the radius should be the same, but here, the value of $a$ is half of the value of $b$. During scratching only the frontal half of the scratch pin is in contact, therefore the value of radius $a$ is compensated by a factor 0.5 , as described in more detail in [20]. Using these data and equation (2.18) for the calculation of $\Lambda$, equation (3.3) gives a temperature rise of $0.88 \mathrm{~K}$. In this estimation, omitting the convection and the thermal conductivity of the scratch pin, it becomes clear that the thermal effect is negligible. From this thermal analysis, it follows that thermal effects are not the reason for material transfer in the case of aluminium sheet. In practice, the used lubricant is used as a deep drawing oil for uncoated and zinc coated steel sheet. Probably, the oil cannot attach strongly enough to the aluminium surface, so it is not able to form a boundary layer between the scratch pin and the sheet material. From these experiments it follows that a proper lubricant is essential to avoid material transfer, [20].

\subsubsection{Unlubricated experiments}

In section 3.3.2 experiments are presented for lubricated contacts. Experiments with the same conditions as in section 3.3.2 are performed, but now with unlubricated contacts. 
Only the degreasing handling of the samples is done as given in section 3.2.3. Like in section 3.3.2, these experiments are performed on zinc coated steel sheet and on aluminium sheet material.

In the case of a degreased surface a strong adhesion can occur between the contacting bodies. Due to the plastic deformation, oxide layers may break, which result in a direct contact between metals and hence even in a stronger adhesion. Assuming that, due to these effects, the contact interface has the same shear strength as the softest contact body, then $f_{H K}=1$. Due to the pin geometry $\theta \leq 45^{\circ}$. For these conditions of $f_{H K}$ and $\theta$, the contact is in the wedge regime according to figure 2.10 .

\section{Aluminium sheet}

The set of experiments with unlubricated aluminium sheet are comparable with the set of experiments in the lubricated case. Beside the use of scratch pins with a tip radius of $50 \mu \mathrm{m}$ also samples with a tip radius of $100 \mu \mathrm{m}$ have been used.

The results of the experiments are presented in a number of steps. First, two experiments are presented that both show material transfer to the scratch pin, but the amount of transferred material and the geometry are totally different, although the conditions are almost the same. Thereafter the effect of scratch length and tip radius will be shown.

The next two experiments that will be shown are performed with a scratch pin with a tip radius of $50 \mu \mathrm{m}$. These scratch pins are loaded with a normal force of $1.25 \mathrm{~N}$. This normal force results in an (initial) attack angle of about $43^{\circ}$.

The first of these two experiments is performed in two steps. In both steps a scratch track is made of $45 \mathrm{~mm}$. After each track an image is made of the scratch pin using SEM. These images are given in figures 3.9 and 3.10 . 


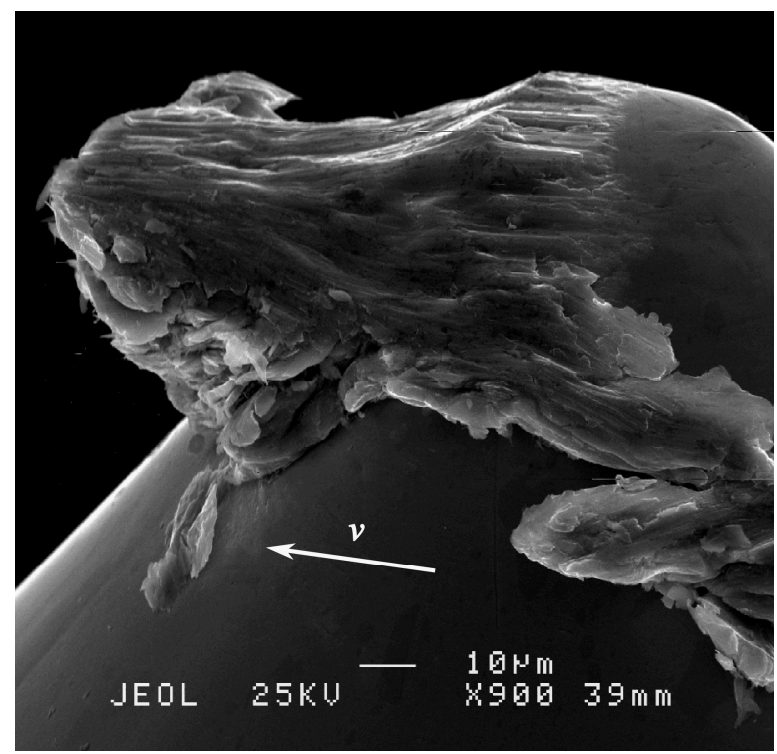

Figure 3.9. SEM picture of a scratch pin of WN1.2379 after $45 \mathrm{~mm}$ of sliding with transferred aluminium from AA5182 EDT sheet material. The very smooth parts are the polished surface of the scratch pin, the rough surface is from the transferred aluminium. The moving direction of the pin is given by the arrow. 


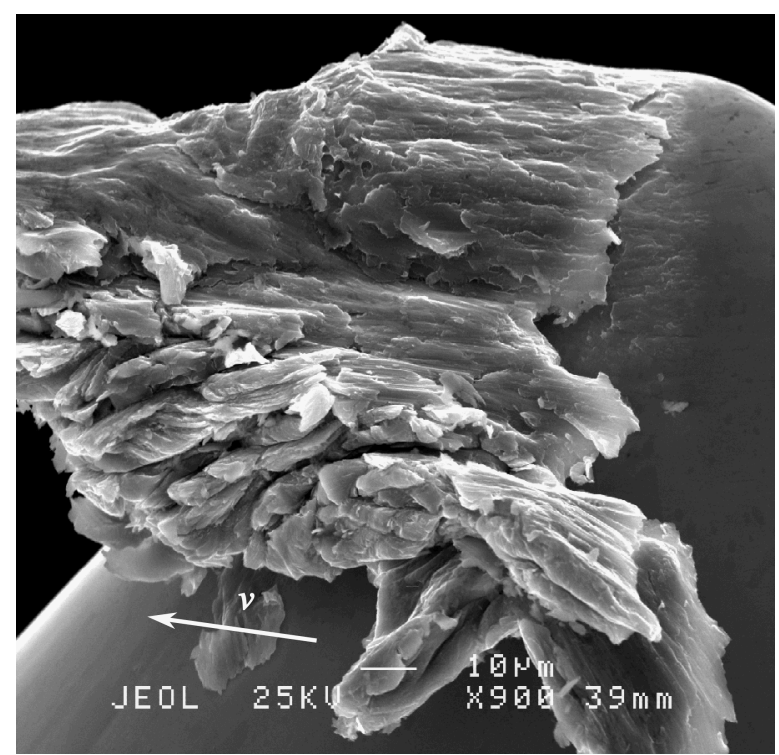

Figure 3.10. SEM picture of a scratch pin of WN1.2379 after $90 \mathrm{~mm}$ of sliding with transferred aluminium from AA5182 EDT sheet material. The very smooth parts are the polished surface of the scratch pin, the rough surface is from the transferred aluminium. The moving direction of the pin is given by the arrow.

In the figures 3.9 and 3.10 it can be observed how a lump develops on the scratch pin. The lump grows slightly in height, but mostly in frontal direction. A schematic view of this lump development is shown in figure 3.11. Due to the lump growth in frontal direction the attack angle changed significantly during scratching. For the case as given in figure 3.9, the attack angle $\theta$ is changed from about $43^{\circ}$ to about $4^{\circ}$. The lump growth in height $\Delta s$ is about $1.5 \mu \mathrm{m}$. For the situation given in figure $3.10 \Delta s$ is increased to about $10 \mu \mathrm{m}$. So, two effects can be seen. At first, the growth in height is dependent on the sliding length and secondly, the attack angle changed in the initial stage of the lump growth process. 


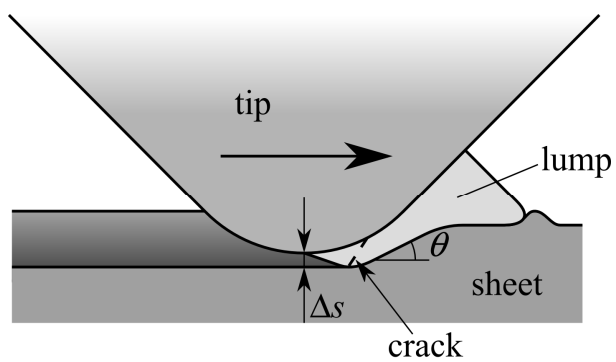

Figure 3.11. Schematic view of lump formation during the scratch experiment. The lump grows in height with $\Delta s$ and in frontal direction, with the consequence that a new contact angle $\theta$ is formed. The arrow indicates the moving direction of the scratch pin.

In the second experiment, again a scratch track is made and an image is made with the SEM, see figure 3.12, but now the result looks really different compared to the figures 3.9 and 3.10. In the experiment shown in the figures 3.9 and 3.10 a lump develops on the scratch pin. However, the galling process is a dynamic process consisting of lump growth, but also of breaking off of these lumps. This effect can be observed in figure 3.12.

Comparing figure 3.12 with 3.9 a huge difference can be observed, when focussing on the amount of material that is transferred. On both figures it is clear that only the frontal part of the scratch pin comes into contact. The contact region is in figure 3.9 the left half and in figure 3.12 this is the right half, but this difference is caused by the different orientation in the SEM. To investigate the difference in transferred material to the scratch pin the sheet is also taken into account. In figure 3.13 a photograph is shown of the sheet made by a confocal microscope. In this figure the scratch track is in line with the dashed line. At the bottom side of the dashed line the end of the scratch track is visible and in upward direction the wear debris that is pushed in front of the scratch pin. The height profile of the dashed line is given in figure 3.14 with the scratch pin fitted in the profile. Most of the material that is transferred to the scratch pin according to figure 3.9 remains on the sheet in the case shown in figure 3.12. Only a small fraction adheres strongly enough to transfer to the pin. During scratching, the wear debris of the sheet material somehow adhered to the scratch pin and peeled off when the pin was lifted from the sheet material. A trace of this peeling off can be found on the profile at $27 \mu \mathrm{m}<x<50 \mu \mathrm{m}$, where the profile overlaps the scratch pin. This overlap originates from material that is pulled in upward direction. The crack that is formed below the surface cannot be measured and is therefore not shown in the profile. During lifting the pin from the sheet, the transferred lump breaks at the dashed line on the lump as shown in figure 3.11. 


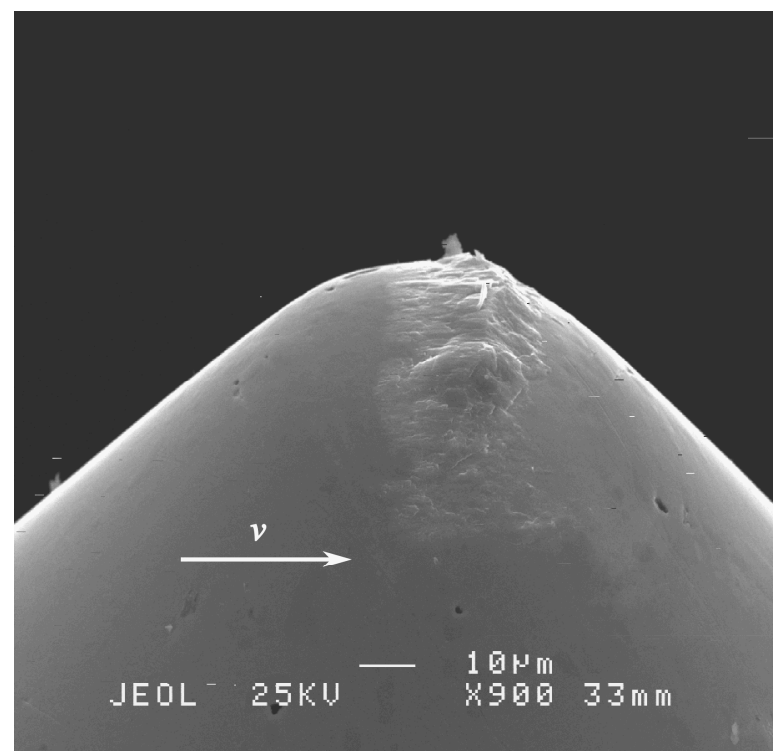

Figure 3.12. SEM picture of a scratch pin of WN1.2379 after $20 \mathrm{~mm}$ with transferred aluminium from AA5182 EDT sheet material. The very smooth parts are the polished surface of the scratch pin, the rough surface is from the transferred aluminium. The moving direction of the pin is given by the arrow.

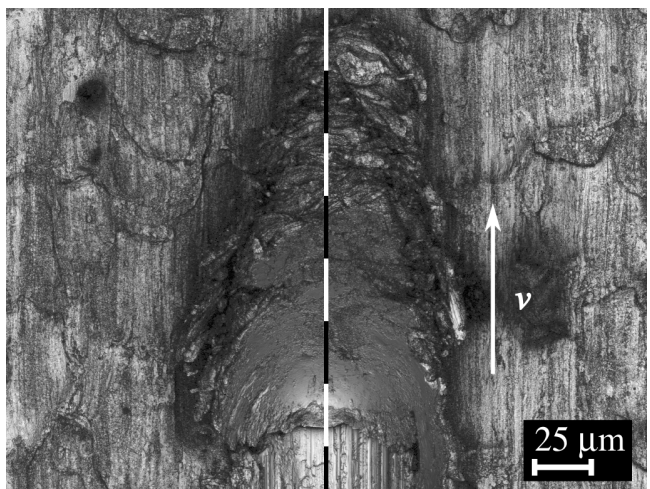

Figure 3.13. A photograph made by a confocal microscope of the AA5182 EDT sheet material. At the bottom side of the dashed line is the end of a scratch track with the scratch pin that is shown in figure 3.12. Around the dashed line in upward direction is wear debris that is pushed in front of the scratch pin. The moving direction of the pin is given by the arrow. 


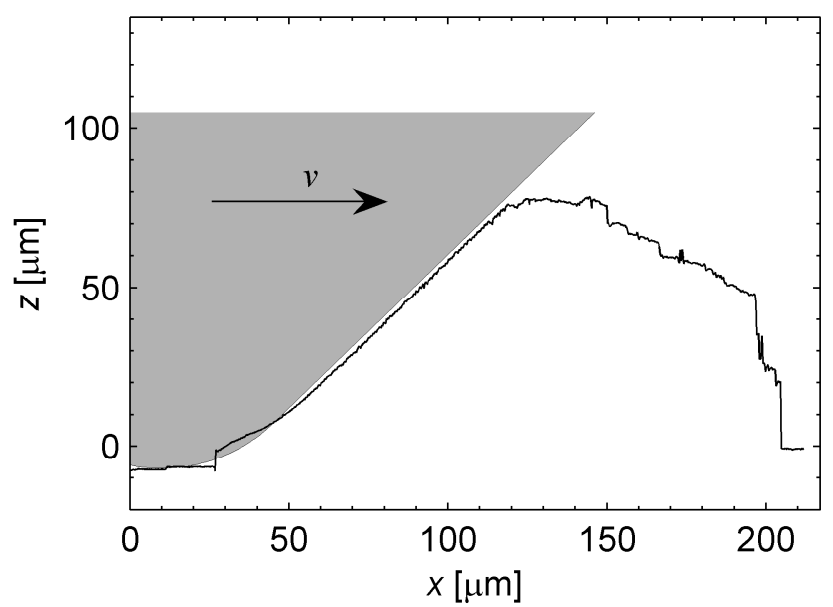

Figure 3.14. Height profile belonging to the dashed line on the surface of figure 3.13 (graph) with the scratch pin fitted on the profile (grey area). The moving direction of the pin is given by the arrow.

In the first experiments with unlubricated aluminium sheet that are presented above, an impression is given of the dynamic behaviour of the lump development. In almost every experiment that is performed, the lump breaks from the scratch pin as shown in figure 3.12. Now, the effect of the length of the scratch track will be investigated. Again, experiments are performed with a scratch pin with a tip radius of $50 \mu \mathrm{m}$, which is loaded with a normal force of $1.25 \mathrm{~N}$. The track lengths are 1,2, 10 and $20 \mathrm{~mm}$. Optical microscope pictures of these experiments are shown in figure 3.15.

Although most material breaks from the pin during lifting from the sheet material, it follows from figure 3.15 that a longer scratch track results in more material transfer. From these experiments, it is difficult to find a well defined relation between the scratch track length and the amount of material transfer. The effect of more material transfer due to a longer scratch length should be interpreted as a trend. Sometimes large deviation from this trend can be found. Such a deviation can be found in the following set of experiments, where a tip radius of $100 \mu \mathrm{m}$ is used. 


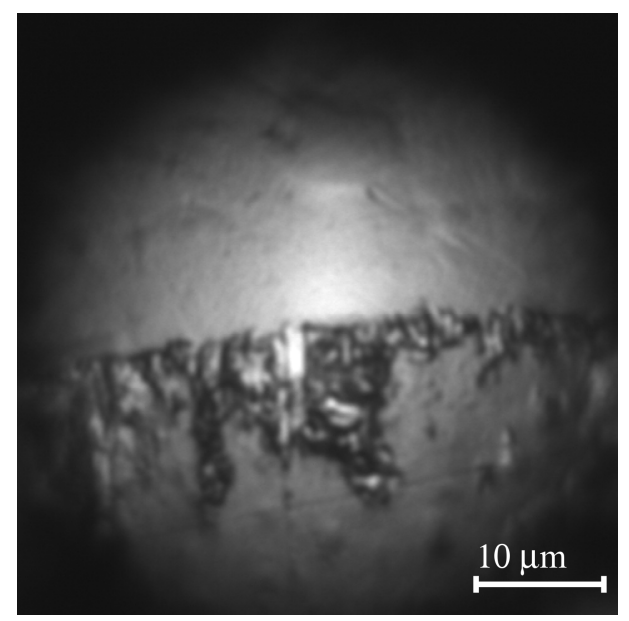

$a$

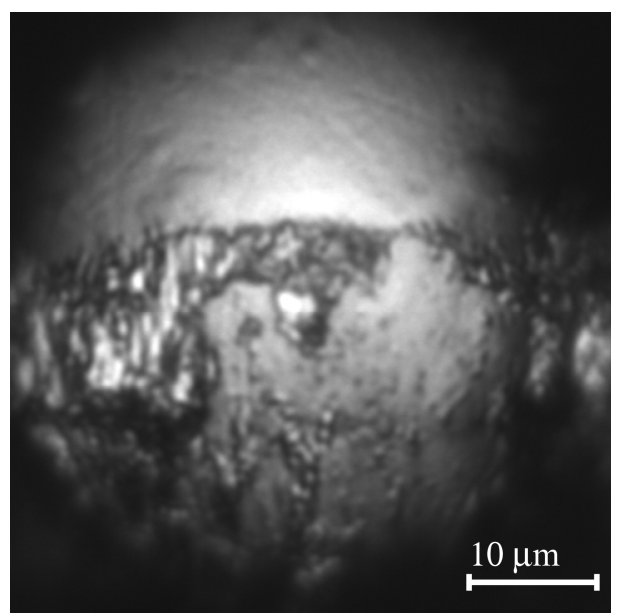

c

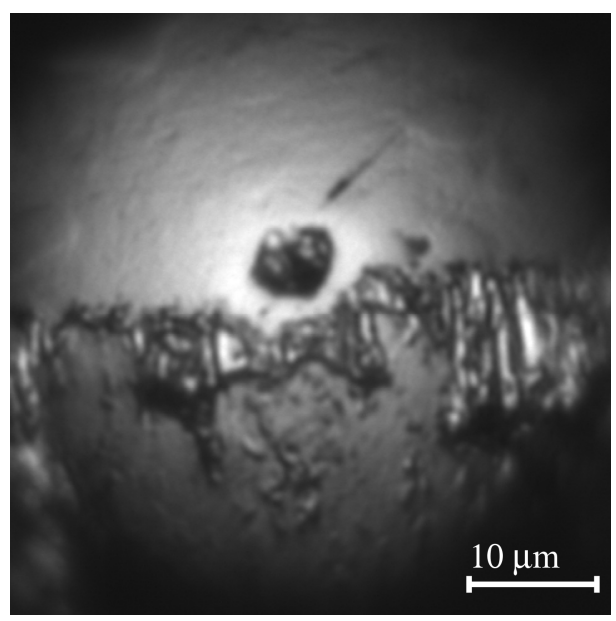

b

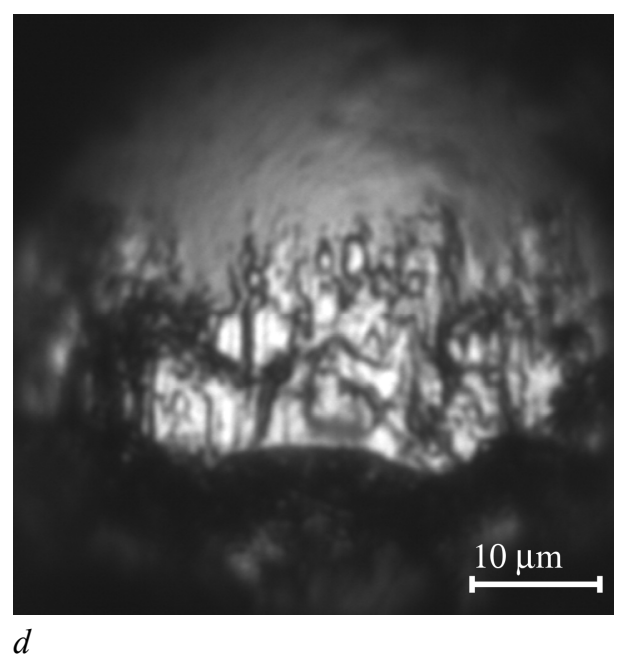

Figure 3.15. Optical microscope pictures of a scratch pin of WN1.2379 with a tip radius of $50 \mu \mathrm{m}$ after 1 (a), 2 (b), 10 (c), 20 (d) mm sliding with transferred aluminium from AA5182 EDT sheet material. The very smooth parts are the polished surface of the scratch pin, the rough surface is from the transferred aluminium. The pins moved in downward direction.

The last set of experiments with aluminium sheet will show the effect of the tip radius of the scratch pin. Now, scratch pins are used with a tip radius of $100 \mu \mathrm{m}$, which is loaded with a normal force of $5 \mathrm{~N}$. The radius of the tip is increased with a factor two, so the normal force has to increase with a factor four to reach the same attack angle. The track 
lengths are 1, 2, 10 and 20, 45 and $90(2 \times 45) \mathrm{mm}$ respectively. Optical microscope pictures of these experiments are shown in figures 3.16 and 3.17 .

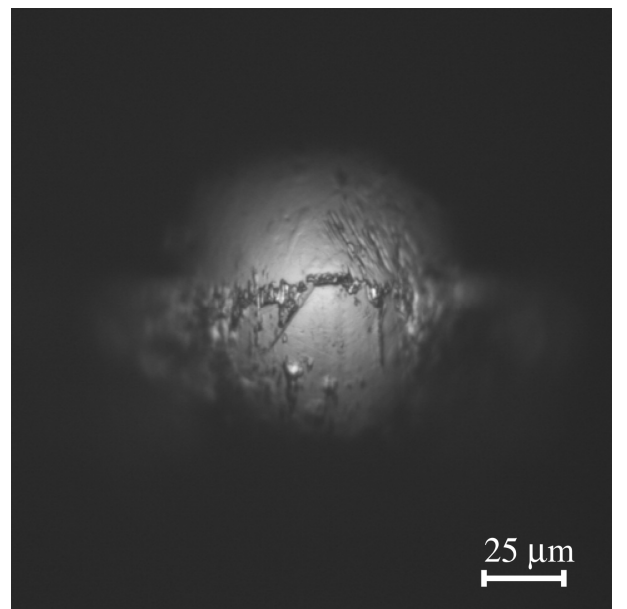

a

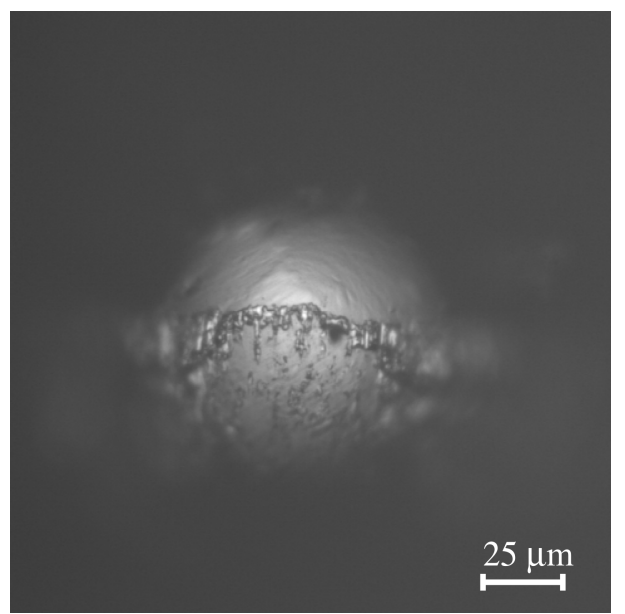

C
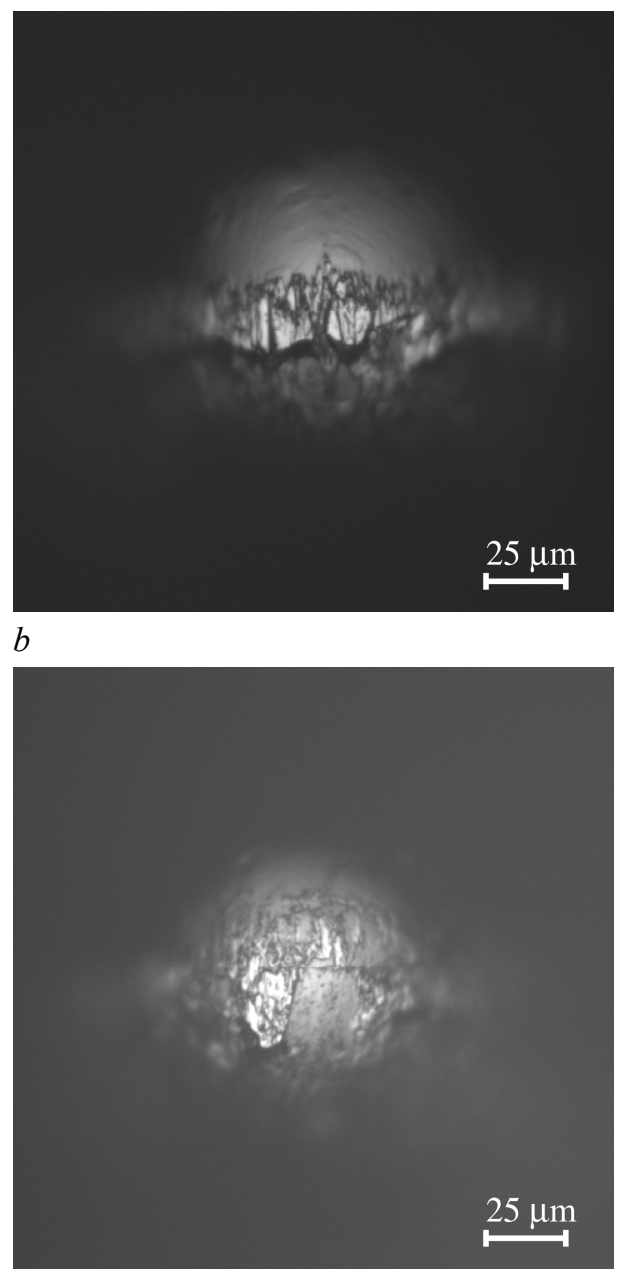

$d$

Figure 3.16. Optical microscope pictures of a scratch pin of WN1.2379 with a tip radius of $100 \mu \mathrm{m}$ after 1 (a), 2 (b), 10 (c), 20 (d) mm of sliding with transferred aluminium from AA5182 EDT sheet material. The very smooth parts are the polished surface of the scratch pin, the rough surface is from the transferred aluminium. The pins moved in downward direction. 


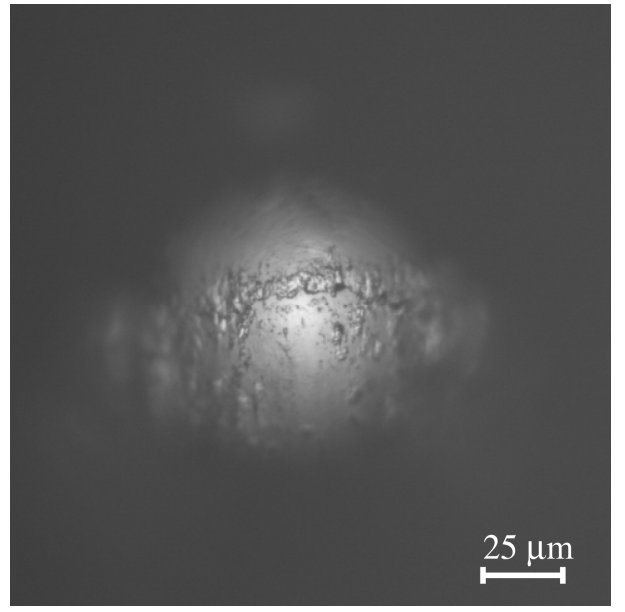

$a$

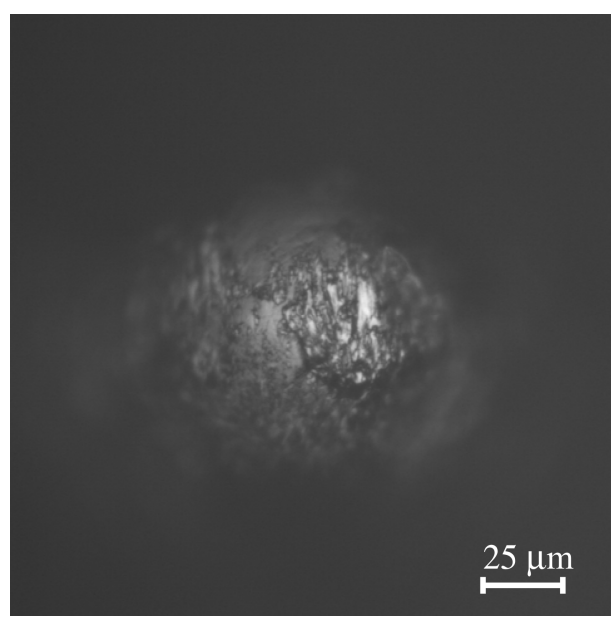

$b$

Figure 3.17. Optical microscope pictures of a scratch pin of WN1.2379 with a tip radius of $100 \mu \mathrm{m}$ after 45 (a), 90 and $(2 \times 45)$ (b) mm of sliding with transferred aluminium from AA5182 EDT sheet material. The very smooth parts are the polished surface of the scratch pin, the rough surface is from the transferred aluminium. The pins moved in downward direction.

For the experiments performed with a scratch pin with a tip radius of $100 \mu \mathrm{m}$, most of the material breaks from the pin during lifting from the sheet material, like in the experiments with the pins with $50 \mu \mathrm{m}$ tip radius.

A clear difference can be observed between the behaviour of the experiments with 50 and $100 \mu \mathrm{m}$ tip radius. For some scratch lengths, the amount of transferred material is of the same order, when focussing on the fraction of the contact area that is covered. But for other scratch lengths, the amount is different. This effect is clearly visible in figure $3.17 \mathrm{a}$, where after $45 \mathrm{~mm}$ of scratching almost no transferred material is left on the pin. But for the cases of about half of the scratch length (20 mm, figure 3.16d) and the double scratch length (90 $\mathrm{mm}$, figure $3.17 \mathrm{~b}$ ) the amount of transferred material is more or less in agreement with the trend that can be seen in the experiments with the $50 \mu \mathrm{m}$ tip radius.

\section{Zinc coated steel sheet}

A number of experiments with unlubricated zinc coated steel sheet are performed. Experiments performed with scratch pins with $50 \mu \mathrm{m}$ tip radius are presented. The scratch pins are loaded with a normal force of $1.25 \mathrm{~N}$. For this set of experiments, only the track length is varied. 
Before showing other results of these experiments, a SEM picture of a scratch pin with transferred zinc material is shown in figure 3.18. It is clear from this figure that the material transfer of zinc behaves differently than is the case with aluminium. The area of the tip that was in contact becomes visible, as seen by the dark print on the surface. The material remaining on the pin after it has been lifted from the sheet material is now situated at the parts of the contact area with a large local attack angle instead of at the top of the pin.

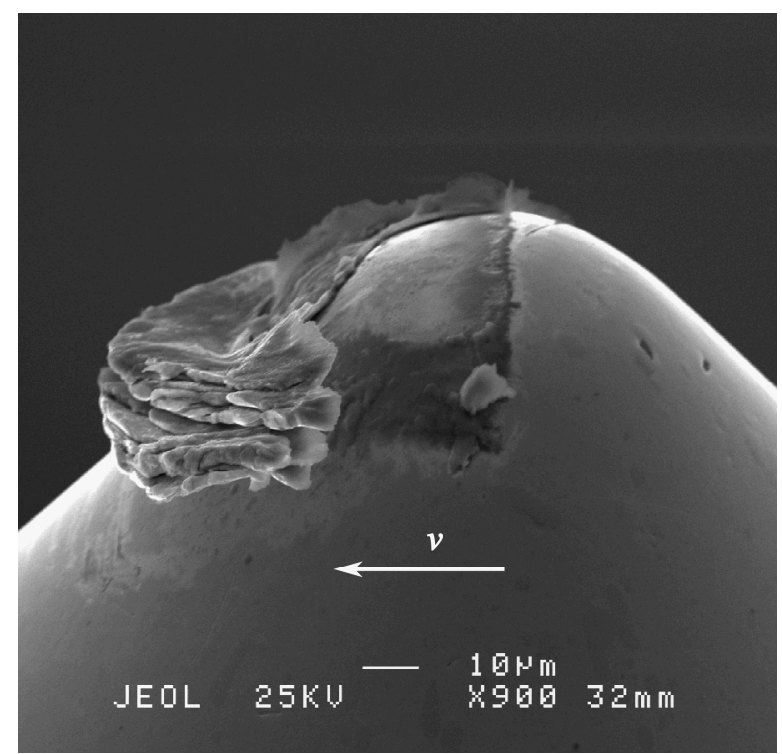

Figure 3.18. SEM picture of a scratch pin of WN1.2379 after $10 \mathrm{~mm}$ of sliding with transferred zinc from DX54D EDT sheet material. The very smooth parts are the polished surface of the scratch pin, the rough surface is from the transferred zinc. The moving direction of the pin is given by the arrow.

A number of experiments have been performed in which the scratch length is varied. Three of the used scratch pins are shown in figure 3.19. The pin used for the experiment of $1 \mathrm{~mm}$ scratch length is omitted, because no material could be observed on it. The pin shown in figure $3.19 \mathrm{~b}$ is as shown in figure 3.18. In figure 3.19a the same effect can be observed as described above for the pin shown in figure 3.18, namely that no zinc is at the top, only at the region with a large local attack angle. The pin shown in figure 3.19c has transferred zinc on the whole area that was in contact. This zinc layer has a geometry as shown in figure 3.11. So, also in the case of zinc coated steel a new effective attack angle $\theta$ is formed due to the transfer of zinc to the tool steel material. From a height measurement it follows that $\Delta s$ is about $0.4 \mu \mathrm{m}$ and $\theta$ is $2^{\circ}$ in the area indicated with $A$ and $8^{\circ}$ in the area indicated with $B$. 


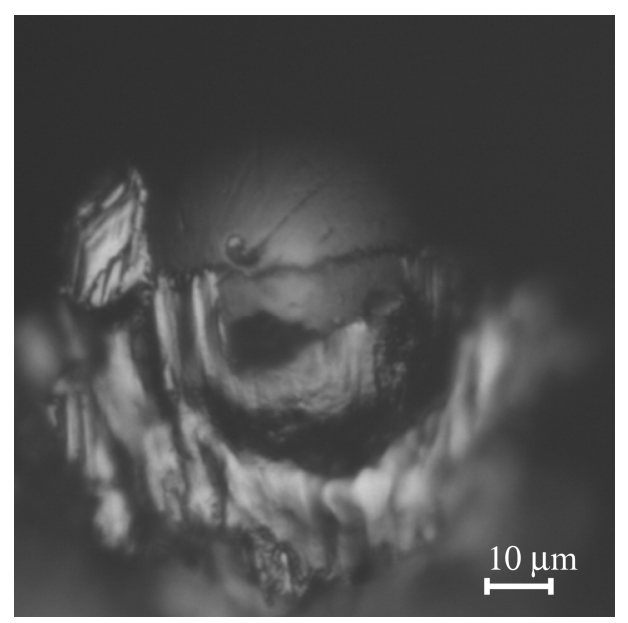

$a$

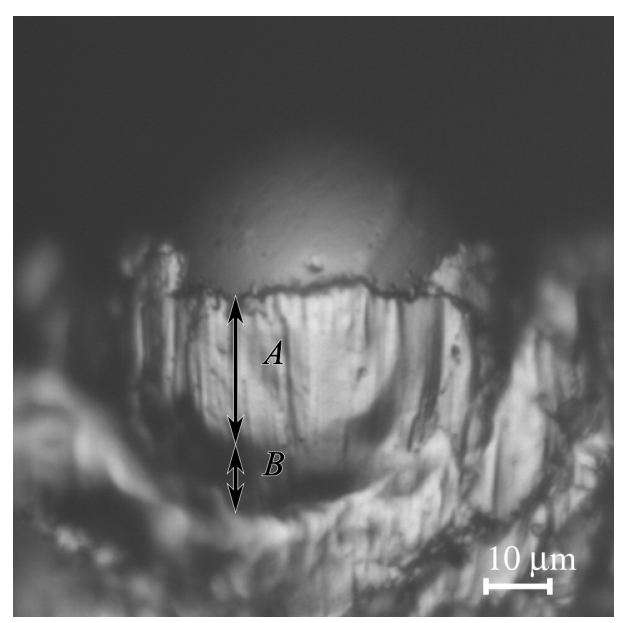

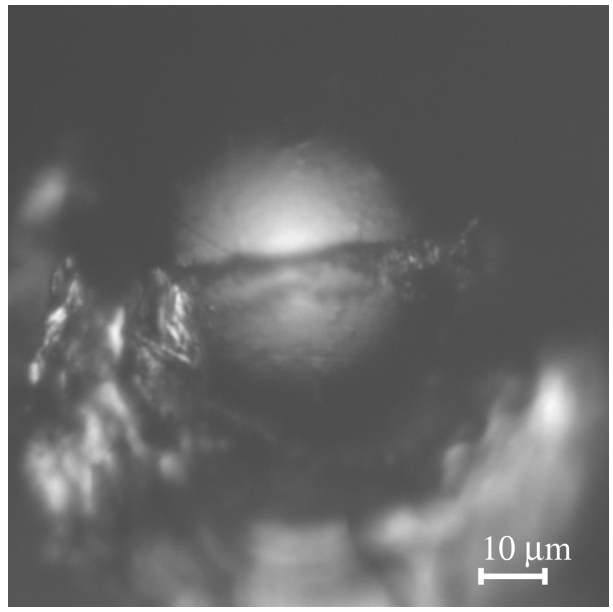

$b$

c

Figure 3.19. Optical microscope pictures of a scratch pin of WN1.2379 with a tip radius of $50 \mu \mathrm{m}$ after 2 (a), 10 (b), 45 (c) mm sliding with transferred zinc from DX45D EDT sheet material. The very smooth parts are the polished surface of the scratch pin, the rough surface is from the transferred zinc. The pins moved in downward direction.

\section{Discussion of the unlubricated experiments}

For the unlubricated case, material transfer is observed in the experiments with aluminium sheet and zinc coated sheet. The effect of lubrication becomes clear, because zinc did not transfer in the lubricated experiments. Besides, aluminium adheres better to the scratch pin, 
because the transferred aluminium peels less easily for the unlubricated case compared with the lubricated one.

As long as the adhesion is not optimal between the transferred material and the scratch pin, a difference can be observed in the way it breaks off from the pin. Once good adhesion is formed between the pin and transferred material, a similar kind of behaviour can be observed for both materials that are tested. A lump will be formed that grows in height and has an attack angle deviating from the original one, as shown in figure 3.11, which looks like a kind of equilibrium angle between the lump that forms and the plastic pressure of the deforming material.

\subsection{Conclusions}

- With the low speeds, when thermal effects still do not play a role, the lubricant protects the tool against direct contact under the condition that the lubricant is compatible with the contacting surfaces. No material transfer has been found for lubricated zinc coated sheet. It appears that the lubricant used, attached not well to the surface of AA5182 EDT to form a protective layer.

- Transferred material is connected to sheet and tool during transfer. During lifting of the pin after the test from the sheet, material breaks at the shear layer inside the sheet material, but also on the interface between tool material and sheet material. When it breaks on a shear layer inside the sheet material a huge amount of material is transferred, otherwise only a fraction or totally no material is transferred.

- For the unlubricated contacts a lump grows in height and in frontal direction. Due to this growth a new attack angle is formed in the contact. The new attack angle is in the order of $4^{\circ}$ for aluminium and varying from $2^{\circ}$ to $8^{\circ}$ for zinc.

- A trend can be observed that for longer scratch tracks more material transfers from the sheet to the tool. Individual situations can strongly deviate from this trend.

- A larger radius of the tip of the scratch pin results in greater deviation in the amount of transferred material. 


\section{Material transfer and lump formation on a single asperity}

\subsection{Introduction}

The phenomenon of galling is a dynamic process of lump growing and of lumps breaking off. In this process a number of steps can be distinguished. First, lumps grow on the tool material due to material pick-up from the sheet material. The lumps grow on the tool due to adhesion or mechanical locking on the interface. After formation of the lump, it has to resist the forces acting on it. Second, if the formed lumps are strong enough they can incur the risk of scratching in the formed products.

In this chapter the focus is on the possibility that material transfers from the sheet to the tool and that the transferred material forms a lump that can resist the forces that will act on it. Models are derived for different cases of the material transfer. In section 4.2 the effect of interlocking due to sharp peaks is presented by a plastic upper bound model. The model is based on a pyramid shaped asperity. The model shows under what conditions material transfer can occur even in the case of lubrication, so a low strength of the interface. This model is applicable for material that is supported by an asperity, but cannot exceed the height of the original asperity, because it needs its strong 'back'. In section 4.3 and following sections, different aspects are presented that are used to formulate a lump growth model which is able to deal with lump growth in height. Further, it is implemented in such a way that it can deal with anisotropic surface geometries as is the case in many grinded tool surfaces. To deal with this geometry, an ellipse shaped asperity is formulated as a

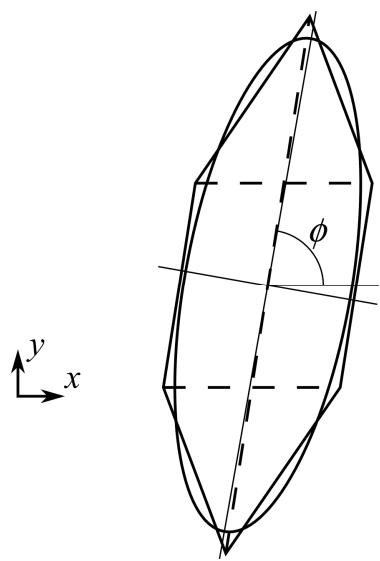

Figure 4.1. An hexagon fitted through an ellipse shaped base of an asperity. 
polyhedron with a hexagon base. In figure 4.1 it is shown how this hexagon base fits a certain asperity with an ellipse shaped base. The model is based on mechanical stability of the formed lump. In section 4.3 the stability analysis is presented, based on the external and internal stresses of a rigid pyramid ploughing through a plastic deforming material. This section focuses on a pyramidal lump for reasons of simplicity. In section 4.4 adhesion is discussed. In section 4.5 , in the first part of the section, the relation between adhesion and the lump growth volume is presented. In the subsequent subsection, the deposition of this volume is presented and in the last part the final geometry is determined on the basis of mechanical stability of the lump.

In this chapter only a single asperity situation will be discussed. On a real surface, this takes place on a surface composed of a large number of asperity contacts. The translation to the multi asperity situation will be discussed in chapter 5 .

\subsection{Interlocking on sharp peaks}

Interlocking is one of the reasons that makes material transfer possible. Interlocking of material takes place especially on sharp edges, such as edges of a scratch, or on asperities with steep slopes due to wear or manufacturing process.

In this section, the case will be studied of a hard asperity ploughing through a plastically deforming and potentially adhering material. Of special interest is the phenomenon that a dead metal zone can be formed in front of the ploughing asperity. This can happen if this is energetically more favourable and is relevant for the similar case of a tool asperity ploughing through a sheet in a deep drawing process.

\subsubsection{Model background}

In this section a model will be formulated of a rigid pyramid shaped body, ploughing through a plastically deforming material. The model will be based on a plasticity model that uses an upper bound method. In this method a kinematic consistent plastic deformation field is defined. In general, a consistent deformation field has to be found, that consumes the minimal amount of deformation power. A simplified deformation field is formulated with a limited number of degrees of freedom. The values of these degrees of freedom are found by minimization of the deformation power that gives the best possible approximation for this simplified case. This strategy is presented by De Vathaire [45]. The model described in [45] will be used as a basis. In this model a rigid pyramid with a square base ploughs through an ideal plastic material, with an edge of the pyramid pointing in the ploughing direction. The geometry of the asperity and the deformation field is presented in figure 4.2. In [45] only one angle $\alpha$ is used. The distinction in $\alpha_{f r}$ and $\alpha_{s}$ will be used in the model discussed in section 4.2 .2 , to replace the square base of the pyramid by a more 
general rhombus. The minimization of the deformation power determines the position of the vertices $A$ and $C$. With the location of these vertices the plastic flow field is determined.

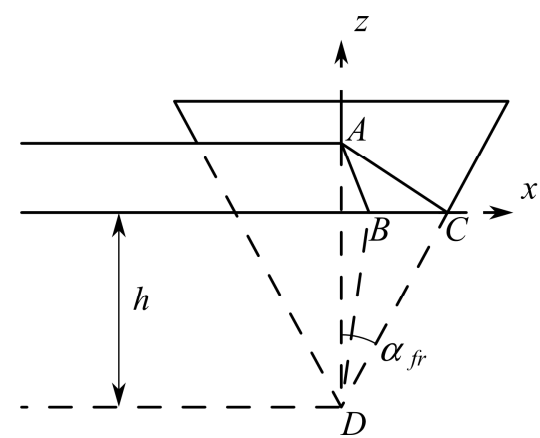

$a$

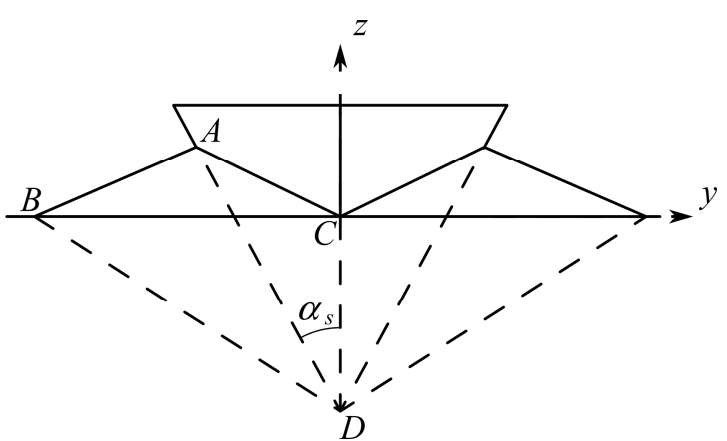

$b$

Figure 4.2. Deformation field of the model of De Vathaire [45], its side view (a) and front view (b).

Atkins [1] presents an alternative geometry for the ploughing body. There, the object that ploughs has a face in the ploughing direction. It results in a blunt front of the ploughing object, see figure 4.3. A dead metal zone $L M L ' Q$ in front of face $L M L^{\prime}$ acts as a 'sharp' tool. (The naming of the vertices is in agreement with [1]. The deviation compared to figure 4.2 has no special meaning.) This model has an extra degree of freedom that has to be optimized. Beside the geometry of the shoulder, that is determined by the vertices $L$ and $P$, the location of $Q$ has to be found. This is done in the same optimizing process.

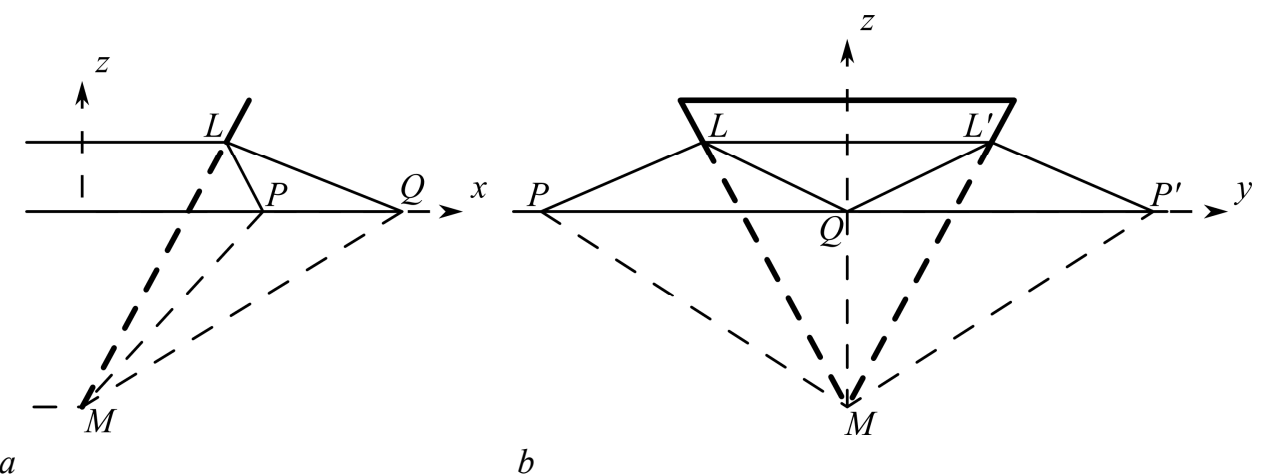

Figure 4.3. Deformation field of the model of Atkins [1], its side view (a) and front view (b). Only the front face of the plough object is given by the thick lines. This face is the only face that is in contact with the plastic deforming material. 
A model will be formulated, using the geometry as presented as given in figure 4.2 combined with the possibility of the formation of a dead metal zone, as is given in figure 4.3. This formulation is given in section 4.2.2.

\subsubsection{Model describing the formation of a dead metal zone}

From experiments presented in chapter 3, it follows that material will be pushed in front of a ploughing indenter as found by Schedin [41], [42]. This effect is modelled by an upper bound model of a rigid pyramid shaped asperity that under certain conditions can push a dead metal zone in front of it. The model of De Vathaire as shown in figure 4.2 is used as the basis. The idea of the dead metal zone as used by Atkins is added to the model, see figure 4.4. The asperity has two top angles: in frontal direction $\alpha_{f r}$ and in sideward direction $\alpha_{s}$. In the model, the angle $\alpha_{s}$ remains constant. The angle $\alpha_{f r}$ has a minimum value $\alpha_{f r 0}$, which is related to the original size of the rigid asperity, but can increase due to the dead metal zone, that acts as an extension of the asperity. From minimization of deformation power, the energetically most favourable situation will be calculated.

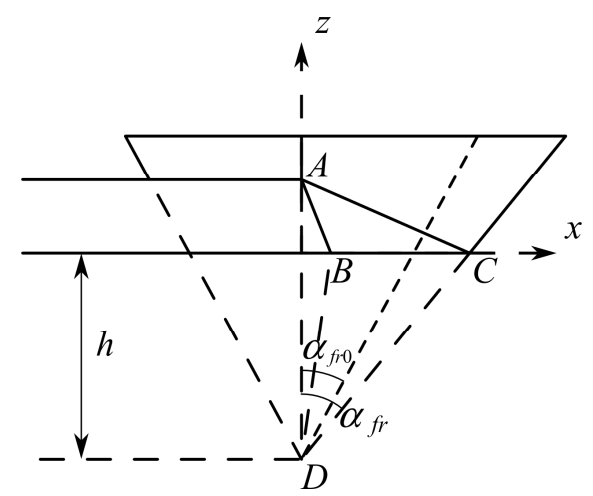

$a$

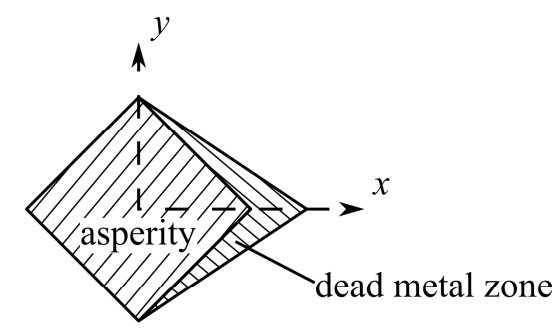

$b$

Figure 4.4. Adapted deformation field of the upper bound model, its side view (a) and a cross section of the asperity and dead metal zone at the xy-plane (b). The field is like the one shown in figure 4.2, but the asperity is 'extended' with a dead metal zone.

In the model that is formulated, the plastic material moves in a set of rigid blocks along the sides of the pyramid. The plastic deformation only occurs at the faces that separate these rigid blocks. The material undergoes shear as it crosses the velocity discontinuity on these planes. In the negative $y$-space (the positive half space will be omitted due to symmetry) the two blocks that describe the deformation are the tetrahedron $A B C D$ and the block of plastic material outside the deformation zone. The faces that divide the blocks and on which the plastic deformation occurs are $A B D$ and $B C D$ as given in figure 4.4. At face $A C D$ power is 
consumed due to friction. The total deformation power is given by a summation of the plastic deformation power and the friction. This results in the following equation:

$$
W=2\left|\vec{u}-\vec{u}_{0}\right| \frac{\sigma_{y}}{\sqrt{3}}\left(A_{A B D}+A_{B C D}\right)+2 m|\vec{u}| \frac{\sigma_{y}}{\sqrt{3}} A_{A C D}
$$

In equation (4.1), $\vec{u}_{0}$ is the velocity vector of the plastic deforming material outside the deformation area and $\vec{u}$ is the velocity vector in the block $A B C D$. The surface areas are given by $A_{X Y Z}$, where the subscripts $X, Y$ and $Z$ give the vertices of the triangular faces. The material parameters are the yield strength $\sigma_{y}$ and $m$. The quantity $m$ is defined as the ratio between the strength of the interface and the shear strength of plastic material, which in the literature is sometimes called the Tresca factor. This factor equals $f_{H K}$ as used in the model of De Rooij [39]. Only terms related to one side of the symmetry plane are given in the equation. To get the total deformation power, the terms are multiplied by a factor of two.

Equation (4.1) is given in a compact form, containing only 'direct' quantities as velocities, surface areas and material parameters. In the calculations the quantities $\vec{u}_{0}, \sigma_{y}$ and $m$ are (taken) constant, the other quantities are variables used in the minimization process. These variables are not independent. The properties and the dependencies of the different variables are given below:

- The equation is formulated in a number of mutually dependent variables. These can be expressed in two independent variables. The minimization process will be performed with the $x$ and $y$-coordinate of vertex $B$.

- Vertices $C$ and $D$ are determined by the indenter geometry and ploughing depth as given by $h$ and $\alpha_{f r}$ in figures 4.2 and 4.4.

- Vertex $A$ is dependent on $\alpha_{s}, h$ and the $y$-coordinate of vertex $D$. Vertex $A$ is located on an edge of the ploughing indenter, the location on this edge is determined by volume conservation. The volume of the material that comes out of the scratch groove should be equal to the volume of the formed shoulders. That means that the area of the cross sections of the groove and shoulders perpendicular on the $x$-axis should be equal. Mathematically, using the unit vector of the $x$-axis $\vec{x}$, this can be written as:

$$
(\overrightarrow{D C} \times \overrightarrow{D B}) \cdot \vec{x}=(\overrightarrow{D A} \times \overrightarrow{D B}) \cdot \vec{x}
$$

- The direction of $\vec{u}$ equals the direction of the line $C A$. As a consequence of this direction, material flows only into the block $A B C D$ through face $B C D$ and leaves it through face $A B D$. The magnitude of $\vec{u}$ is determined by volume conservation of plastic material. The material flows outside the deformation zone with velocity $\vec{u}_{0}$. This flow is in negative $x$-direction according to the coordinate system in 
figures 4.2 and 4.4. The volume flow should be the same at both sides of face $B C D$. This is also the case for face $A B D$, but the volume conservation does not have to be implemented for the latter face, because this follows implicitly from volume conservation at face $B C D$ and the dependency of the location of vertices $A$ and $D$. Using the normal of face $B C D \vec{n}_{B C D}$, the magnitude of $\vec{u}$ can be calculated with the following equation:

$$
\vec{n}_{B C D} \cdot \vec{u}=\vec{n}_{B C D} \cdot \vec{u}_{0}
$$

Following the steps given above from equation (4.1) an objective function is obtained of two independent variables that is minimized by the simplex method. This function is used in a dimensionless form by normalizing it with the frontal area of the ploughing indenter (proportional to the moved material from the groove to the shoulders), the scratch velocity and the yield strength of the deforming material. This results in the following function:

$$
\bar{W}=\frac{W\left(x_{B}, y_{B}\right)}{\left|\vec{u}_{0}\right| \sigma_{y} h^{2} \tan \left(\alpha_{s}\right)}
$$

Figure 4.5 shows some results of the model. In this figure, for the angle $\alpha_{s}=45^{\circ}$ graphs of the normalized deformation power are given for different values of $\alpha_{f r}$ as a function of the factor $m$. In figure 4.5 a horizontal dashed line shows the minimal deformation power in the case that $m=1$. The situation with $m=1$ corresponds to the situation of shear in the bulk material $(\tau=k)$. An $m$ factor smaller than 1 can occur when shear at the interface between the ploughing asperity and the plastically deforming material takes place. The situation of minimal deformation power in the case of $m=1$ happens as $\alpha_{f r}=54^{\circ}$. In the case that $m=1$ in the sense of stresses no difference exists between shearing within the plastic deforming material or on the interface between the asperity and plastic deforming material, and so, no preference exists of shearing on the interface or within the plastic material. Taking into account the plastic power, the situations that belong to the area in figure 4.5 above the dashed line will not happen as shear can happen within the material with less power. Shear in the material, which means formation of a dead metal zone, can only happen as that results in an $\alpha_{f r}$ that is larger than $\alpha_{f r 0}$, otherwise the rigid pyramid itself shears. From figure 4.5 it follows, that a dead metal zone is not only formed if $m=1$, but even in the case if $m<1$. 


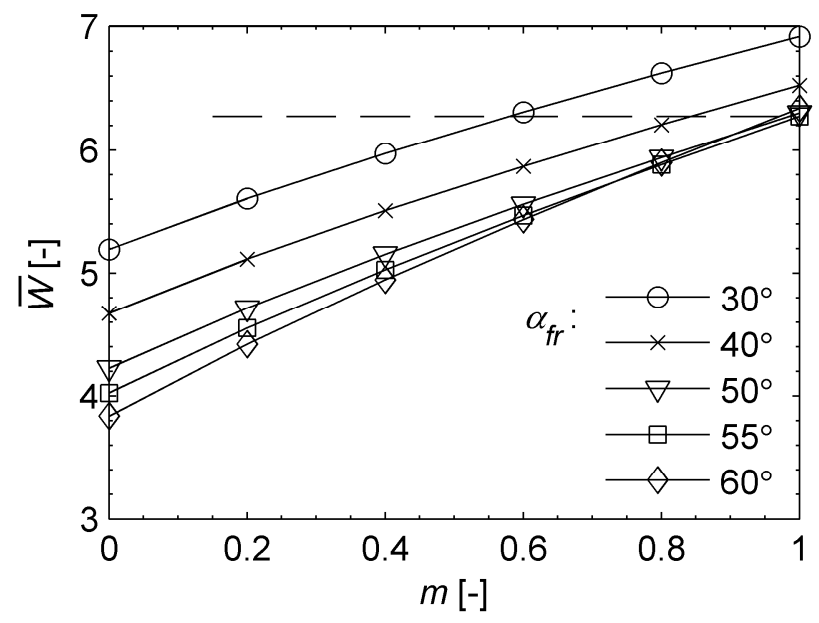

Figure 4.5. Deformation power (normalized) versus Tresca factor $m$ for different values of $\alpha_{f r}$ and $\alpha_{\mathrm{s}}=45^{\circ}$. The horizontal dashed line gives the minimum level of power in the case that $m=1$.

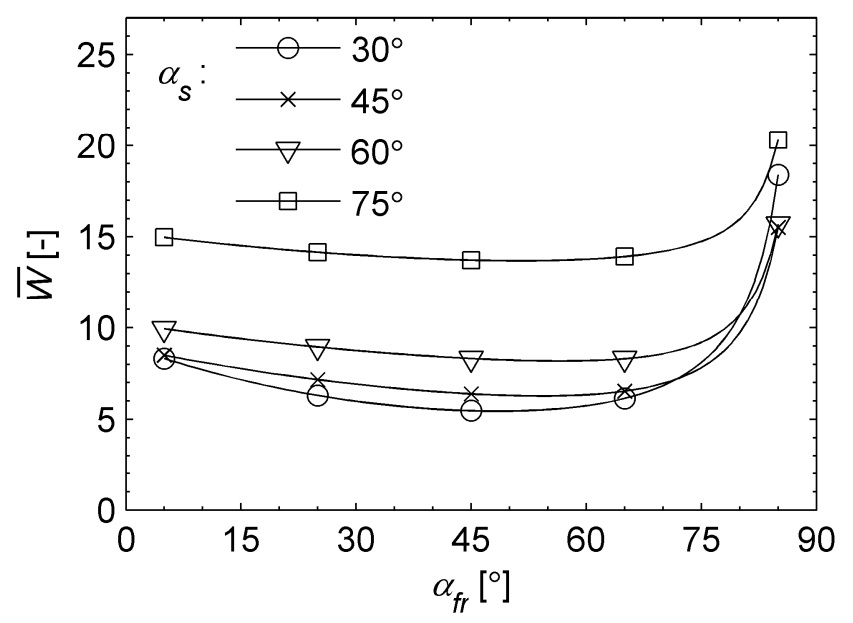

Figure 4.6. Deformation power (normalized) versus $\alpha_{f r}$ for different values of $\alpha_{s}$ and $m=1$.

In figure 4.6 the normalized deformation power is presented as a function of $\alpha_{f r}$ for different values of $\alpha_{s}$. If a dead metal zone is formed, the value of $\alpha_{f r}$ will be the minimum value of the graph. These minimum values of $\alpha_{f r}$ are about $40^{\circ}$ to $55^{\circ}$. 
In figure 4.7, the results of the model are shown in a more general form. In this figure lines are shown that give the transition between shear in bulk and shear on the interface for a certain value of $\alpha_{s}$. Below the transition line shear in the bulk takes place and a dead metal zone will be formed. A transition line in the figure connects situations with an equal deformation power for a certain $\alpha_{s}$. Ploughing with a pyramid with an $\alpha_{f r}$ that is below the graph costs more power than a situation on the graph, so a dead metal zone will be formed. This dead metal zone gets an $\alpha_{f r}$ that can be found by following the graph till $m=1$, due to lack of any lubrication.

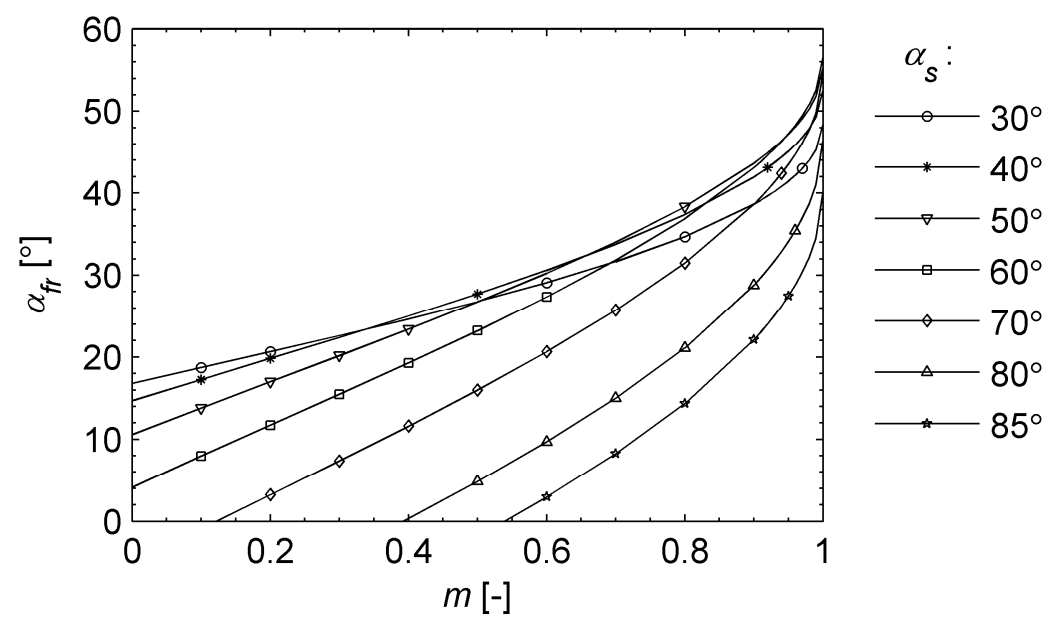

Figure 4.7. Transition lines from shear in bulk material (dead metal zone formation) to shear on interface for different values of $\alpha_{s}$.

From the graphs in figure 4.7 it follows that as the interface is weaker, for example due to lubrication, $\alpha_{f r}$ has to be smaller, to get shear in the bulk. Or in other words, if the surface is better lubricated, a higher attack angle is needed to get shear in the bulk material. Further, dead metal zones are only formed in the case of low values of $\alpha_{f r}$. In general, engineering surfaces do not have such steep roughness peaks, mostly the slopes are far below $30^{\circ}$, so the half top angle, like $\alpha_{f r}$ and $\alpha_{s}$ should be above $60^{\circ}$. In that case no material pickup will happen due to formation of dead metal zones on roughness scale. The steep slopes are the risky zones for material transfer due to the effect given above, for example due to sharp scratches.

The model presented here gives a mechanism for material transfer due to interlocking. It can be shown that material wears off from the plastic deforming contact body and attaches 
to the ploughing pyramid. This wearing off of material can happen even in lubricated cases as can be seen in figure 4.7 for the case that $m<1$ and there is no work hardening.

For the two dimensional case a comparable model is formulated by Avitzur [2]. In two dimensions it is in fact not possible to describe ploughing behaviour. Because of material conservation, the height of the plastic deforming material behind the plastic deformation zone should be the same as in front of this zone or otherwise it is a matter of material removal. In the case of no material removal only rubbing of a ridge can be described. Avitzur shows that on the base of plastic work minimization the attack angle cannot exceed an angle of $14^{\circ}$ in the case of rubbing. Higher attack angles shear off the deforming ridge. This maximum angle of $14^{\circ}$ is only possible as $m=0$. For higher values of $m$ this angle is even smaller. Translated to variables of the model described in this chapter, $\alpha_{f r}$ should exceed $76^{\circ}$ to avoid that material shears off.

In this section the focus was on plastic deforming material. The manner of deformation was determined on the base of work minimization. This gives the possibility to show how roughness of the rigid material in a number of cases is filled with the material from the plastic deforming body. Due to the fact that work hardening is not taken into account, the material that sticks in front of the rigid asperity still needs a 'back' to do not shear off from the asperity. So, growth in height cannot be described by this model. In the following sections the focus is on the asperity and the transferred layer on it. By making force analyses and assuming hardening of the transferred material, a model will be shown that can describe asperity growth in height.

\subsection{Stability criterion for pyramidal shaped asperities}

\subsubsection{Introduction}

The stability of an asperity or a lump that is formed on an asperity will be discussed in this section. On the basis of a mechanical analysis a model is formulated, that estimates the stresses inside a lump at the interface between the lump and the original tool material or within the lump itself. From this analysis it follows whether the lump is stable or it collapses. The parameters in this model are the strength of the lump material, the geometry of the lump and the strength of the interface between the lump and the plastic deforming counter material. In the case where the lump is stable and able to grow, it potentially causes severe scratching of the sheet.

The model is a derivation of the surface flow line model presented by Lafaye [29]. In the surface flow line model, the forces are the result of a normal contact pressure and tangential stresses acting in the direction of an assumed flow line field. For formulating the model in this section, a pyramid shape is chosen. In a lot of models, a spherical shaped asperity geometry is used, such as in the models presented in chapter 2 and in [29]. According to the 
performed experiments, a steady angle can more or less be observed during material transfer from sheet metal material to a scratching asperity, as discussed in chapter 3 , see figure 3.11. The pyramid is a geometry in which a certain attack angle can be easily implemented. Another advantage is the relatively simple mathematical description of this shape.

\subsubsection{Stress analysis of a lump}

An asperity is formulated as a pyramid shaped body as given in figure 4.8 . The geometry as depicted in this figure is used to formulate the statics model and only describes the part of the asperity in contact. The points $B$ and $D$ are respectively the extremes of the width $w$ and the height $h$, so behind the plane $A D B$, no contact is expected. Therefore the geometry behind the plane $A D B$ is omitted first, to simplify the analysis. In reality the internal stress distribution is also dependent of the rear part of the asperity. This extension of the model will be discussed in section 4.3.4. With the geometry given in figure 4.8, the lump is completely defined by $w, h$ and the length $l$ or in the dimensionless form, normalizing by $l$, two geometrical quantities remain: $\bar{h}=h / l$ and $\bar{w}=w / l$.

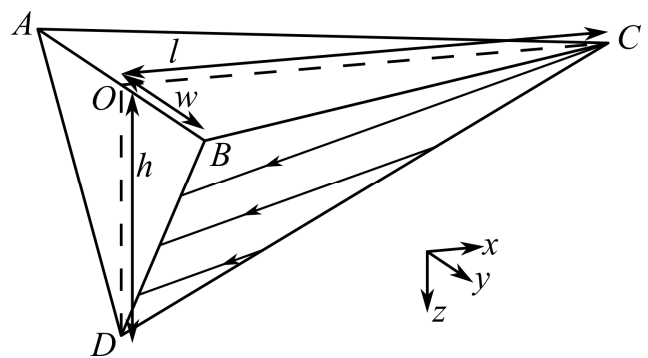

Figure 4.8. Tip geometry.

The following is assumed in the model:

- The coordinate system is defined as given in figure 4.8. The origin $O$ is on the point where the two dashed lines are crossing. The dashed lines are on the $x$ and $z$-axis.

- The tip is stationary. In the far field the plastic material flows in the negative $x$-axis direction.

- The asperity is symmetric. The plane of symmetry is the $x z$-plane. Because of this symmetry, effects that play a role in the positive $y$-space, play implicitly also a role in the negative $y$-space, but will not always be called explicit.

- Face $B D C$ (and $A C D$, because of symmetry) is the contact plane on which the normal pressure and shear stresses act. 
- The direction of the contact shear stress is determined by the surface flow lines of the plastic material in contact with the tip. The direction of the shear stress is determined by the vector in face $B D C$, that minimizes the angle with the flow in the far field, that means, minimize the inner product $\vec{x} \cdot \vec{t}$, with $\vec{x}$ the unit vector in the direction of the positive $x$-axis and $\vec{t}$ the unit vector of the direction of the contact shear stress. The direction given by this minimization does not have to be in agreement with the direction of shear in reality, but this is chosen as an approximation.

- The contact pressure has a constant value $p_{p l}$ on the whole contact area. The tangential shear stress $\tau_{p l}$ is calculated using a Coulomb friction law $\tau_{p l}=\mu p_{p l}$.

- The asperity is rigid.

- The connection of the asperity to the bulk material at face $A B C$ is an elastic spring. This results in a constant tangential stress $\tau_{A B C}$ in the $x$-direction and a linear function for the value normal stress $\sigma_{A B C}$ which is a function of the $x$-coordinate.

Sign conventions used for stresses in the model:

- Plastic pressure $p_{p l}$ is positive in the case of compressive stress.

- Plastic shear stress $\tau_{p l}$ is positive in the direction of $\vec{t}$.

- Normal stress $\sigma_{A B C}$ is positive for tensile stress. This is the opposite of the convention of $p_{p l}$. Usually in mechanics: tensile is defined as positive, but contact pressure deviates from this standard, because of its compressive nature.

- Tangential stress $\tau_{A B C}$ is positive in the positive $x$-direction.

The external forces are acting on the face $B D C$. The coordinates of these three points $B, D$ and $C$ are respectively given by $(0, w, 0),(0,0, h)$ and $(l, 0,0)$. These points describe a plane, which has the unit normal vector:

$$
\vec{n}_{B D C}=\frac{(w h, h l, l w)}{\sqrt{(w h)^{2}+(h l)^{2}+(l w)^{2}}}
$$

To minimize $\vec{x} \cdot \vec{t}$ vector $\vec{t}$ has to be in the plane that is described by the vectors $\vec{n}_{B D C}$. This results in:

$$
\vec{t}=\sqrt{\frac{w^{2}+h^{2}}{(w h)^{2}+(h l)^{2}+(l w)^{2}}}\left(-l, h \frac{w h}{w^{2}+h^{2}}, w \frac{w h}{w^{2}+h^{2}}\right)
$$

These two vectors have to be used in the incremental force equations:

$$
\begin{gathered}
d \vec{F}_{n}=-p_{p l} \vec{n} d A \\
d \vec{F}_{t}=\tau_{p l} \vec{t} d A=\mu p_{p l} \vec{t} d A
\end{gathered}
$$


With equations (4.7a) and (4.7b) the external forces on the asperity are given. These relations are used to calculate internal stresses.

From the tip with the properties as given above, stress relations are found on the basis of a statics analysis. This statics analysis is elaborated in appendix A. The stress relations found are, in dimensionless form (as presented in equations (A.18) and (A.19)):

$$
\begin{gathered}
\frac{\tau_{A B C}}{p_{p l}}=\frac{1}{\bar{A}_{A B C}}\left(\bar{w} \bar{h}+\mu \sqrt{\bar{w}^{2}+\bar{h}^{2}}\right) \\
\frac{\sigma_{A B C}}{p_{p l}}=-\frac{\bar{w}}{\bar{A}_{A B C}}\left(1-\frac{\mu \bar{w} \bar{h}}{\sqrt{\bar{w}^{2}+\bar{h}^{2}}}\right)+\frac{1}{3 \bar{I}_{c y y}}\left(\bar{w}\left(\bar{h}^{2}-1+3 \bar{x}_{c}\right)+\frac{\mu \bar{h}\left(2 \bar{w}^{2}+\bar{h}^{2}-3 \bar{x}_{c} \bar{w}^{2}\right)}{\sqrt{\bar{w}^{2}+\bar{h}^{2}}}\right)\left(\bar{x}-\bar{x}_{c}\right)
\end{gathered}
$$

In these equations $\bar{A}_{A B C}$ is the surface area of face $A B C$, see appendix A equation (A.24), $\bar{x}$ the $x$-coordinate, $\bar{x}_{c}$ the centroid of face $A B C$ and $\bar{I}_{c y y}$ the area moment of inertia of face $A B C$ with respect to the line $\bar{x}=\bar{x}_{c}$. The bar above these quantities indicates that they are in the dimensionless form. Lengths, areas and area moments are normalized by respectively $l$, $l^{2}$ and $l^{4}$. The definition of $A_{A B C}, x_{c}$ and $I_{c y y}$ is worked out in section D.4.

Equations (4.8) and (4.9) can be simplified by substituting $\bar{x}_{c}, \bar{A}_{A B C}$ and $\bar{I}_{c y y}$ by their equivalent functions of $\bar{w}$ and $\bar{x}$. The substitution will be omitted, because the general formulation of equations (4.8) and (4.9) makes it possible to use these equations for other situations, like a modified asperity shape and crack formation. These situations will be further discussed in sections 4.3.4 and 4.3.6.

\subsubsection{Discussion of stress analysis}

For a number of situations the graph is given in figure 4.9 of $\tau_{A B C} / p_{p l}$ as a function of the normalized asperity height $\bar{h}$ by varying the normalized asperity width $\bar{w}$. According to [39] the relation between hardness and yield strength is given by:

$$
H=2.8 \sigma_{y}
$$

In the model, $H$ is the hardness of the plastically deforming material. It is assumed that $p_{p l}$ equals $H$, because $p_{p l}$ is the real contact pressure. According to the Tresca criterion a material fails as the shear stress exceeds a certain value. This maximum shear stress value is $0.5 \sigma_{y}$. From equation (4.10), the assumed value of $H$ and the Tresca criterion it follows that:

$$
\tau_{p l \_m a x}=0.18 p_{p l}
$$

From this analysis it follows that $\mu$ has a maximum value of 0.18 . The coefficient $\mu$ is the coefficient of friction at the interface and contains no ploughing effect. Due to ploughing 
the macroscopic coefficient of friction might be higher. The maximum value of $\mu$ will only occur in the case of a very good adhesion of the plastic material to the asperity. From the graphs in figure 4.9 it follows how strong an asperity has to be to resist the internal shear stresses as a result of the plastic forces acting on the asperity, using the equations (4.8) and (4.11).

From figure 4.9 it becomes clear that $\tau_{A B C}$ is strongly related to $\bar{h}$. The influence of $\bar{w}$ is limited. Although the values of $\bar{h}$ and $\bar{w}$ contribute in the same amount to the plastic forces, a higher value of $\bar{w}$ results in a larger area $\bar{A}_{A B C}$ over which the plastic forces can be distributed. So speaking in terms of stresses, the increasing amount of plastic forces due to the increase of $\bar{w}$ is almost cancelled out by the larger bearing area of $A B C$. The effect of $\mu$ is of importance for values of $\bar{h}<<1$. If $\bar{h}=0$ only adhesive forces act on the asperity, so $\tau / p_{p l}=\mu$ for $\bar{h}=0$. For higher values of $\bar{h}$ the effect of the frontal area, and so the ploughing effect, dominates the plastic forces. 


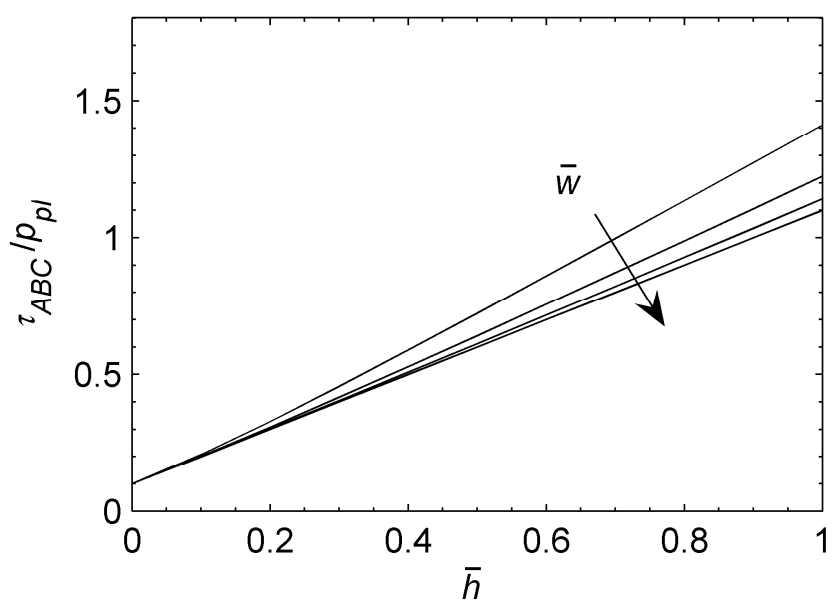

a

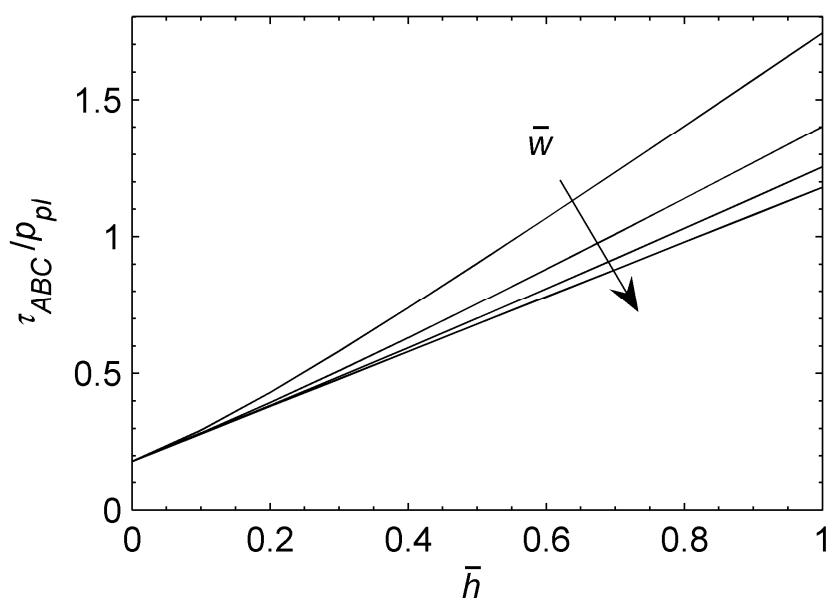

$b$

Figure 4.9. Mean shear stress over plastic pressure as a function of normalized asperity height on face $A B C$ for $\bar{w}=0.25,0.5,1$ and lim $\bar{w} \rightarrow \infty$ for two different plastic shear strengths: $\mu=0.1$ (a) and $\mu=0.18$ (b). The arrows give the order of the graphs for increasing $\bar{w}$.

The stress component $\sigma_{A B C}$ is given by equation (4.9). Equation (4.9) is a linear function of the $\bar{x}$-coordinate, so the extremes of $\sigma_{A B C}$ can be found on the extreme $\bar{x}$-coordinates $\bar{x}=0$ and $\bar{x}=1$. For a number of situations, graphs of $\sigma_{A B C}$ are given for these points in figure 4.10. For $\bar{h}<<1$, from $\bar{x}=0$ to $\bar{x}=1 \sigma_{A B C}$ is about the contact pressure. For larger values of 
$\bar{h}$, the effect of the bending moment with respect to $\bar{x}=\bar{x}_{c}$ becomes more and more dominant. In figure 4.10, this effect follows from the lines of $\bar{x}=0$ and $\bar{x}=1$ that diverge for increasing $\bar{h}$.

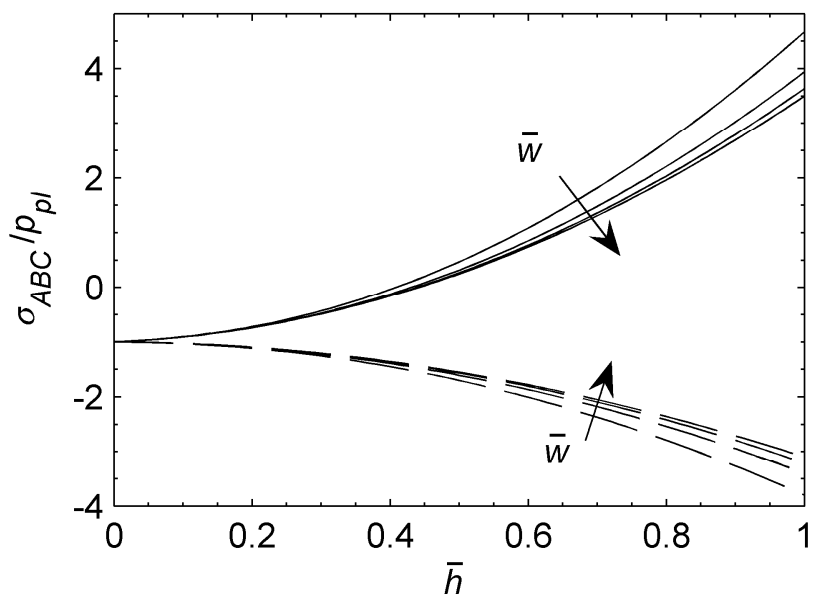

a

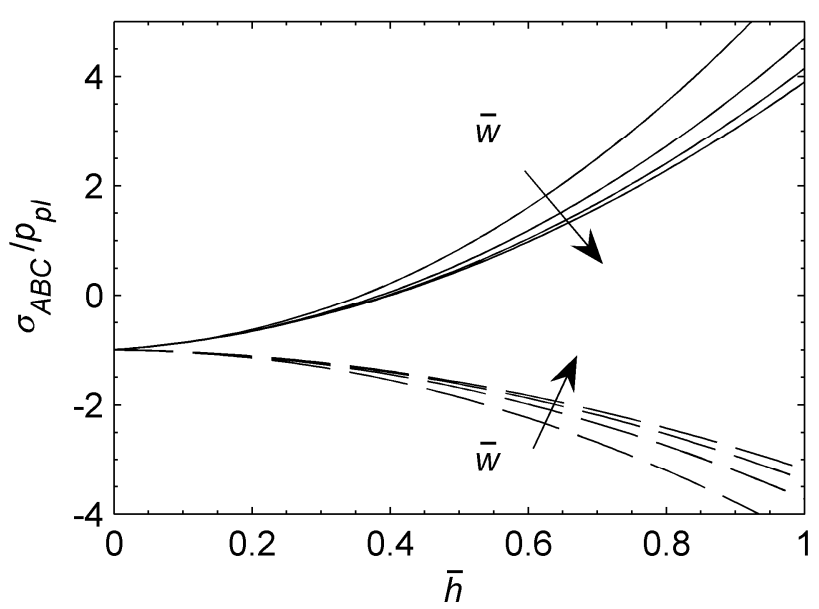

b

Figure 4.10. Normal stress over plastic pressure as a function of normalized asperity height on face $A B C$ at $\bar{x}=0$ (dashed lines) and $\bar{x}=1$ (solid lines) for $\bar{w}=0.25,0.5,1$ and lim $\bar{w} \rightarrow \infty$ for two different plastic shear strengths: $\mu=0.1$ (a) and $\mu=0.18$ (b). The arrows give the order of the graphs for increasing $\bar{w}$. 
For determining the stability of the lump, a stress criterion has to be used. Using the Von Mises yield criterion all the stress components have to be known. In the case where it is assumed that $\tau_{A B C}$ and $\sigma_{A B C}$ are the only relevant stress components, the Von Mises criterion reduces to:

$$
\sigma_{V M}=\sqrt{\sigma_{A B C}^{2}+3 \tau_{A B C}^{2}}
$$

In the case where the bulk material at face $A B C$ is fixed, so $\varepsilon_{x x}=\varepsilon_{y y}=0$ and $\tau_{A B C}$ is the only relevant shear stress component, the Von Mises criterion becomes (using Poisson's ratio $v$ ):

$$
\sigma_{V M}=\sqrt{\left(\frac{1-2 v}{1-v}\right)^{2}{\sigma_{A B C}}^{2}+3 \tau_{A B C}{ }^{2}}
$$

Both components, $\tau_{A B C}$ and $\sigma_{A B C}$, according to equations (4.12) and (4.13), do not make a negligible contribution to the Von Mises stress. Poisson's effect will reduce the effect of $\sigma_{A B C}$, but the effect is still there. In sections 4.3 .4 and 4.3 .5 , the stresses, especially $\sigma_{A B C}$, will be discussed in more detail, in order to formulate a stability criterion for the lump.

\subsubsection{Model with an extended base of the asperity}

The asperity of the model formulated in section 4.3.2 is based on a simplified geometry. The pyramid that has been used has the face $A B C$ as its base, which lies in the $x y$-plane on the $y$-axis and the positive $x$-area. The top $D$ is positioned on the $z$-axis. This simplifies the formulation of the model, but has some shortcomings. In the model the rear face of the pyramid, located on the $y z$-plane, is perpendicular to the base $A B C$, but in general surface roughness does not have very steep faces, even sharp scratches have in general a slope far below $90^{\circ}$. To reduce the steepness of the back face, the base of the pyramid has to be extended into the negative $x$-area on the $x y$-plane.

A manner to adapt the asperity of the current model to a more realistic one can be done by extending the pyramid with its mirrored body, by mirroring it in the $y z$-plane. This results in a pyramid that has extended its base $A B C$ with one extra vertex $C^{\prime}$ with coordinates $(-l, 0,0)$, see figure 4.11 . This extension of the asperity has no consequences for the external plastic forces on it, because the plastic material is only in contact with the faces at the front side of the $y z$-plane (faces $B D C$ and $A C D$ ), which exist already. Due to the extension of the asperity, inside the asperity the plastic forces can be carried on a wider area, which results in reduced stresses. 


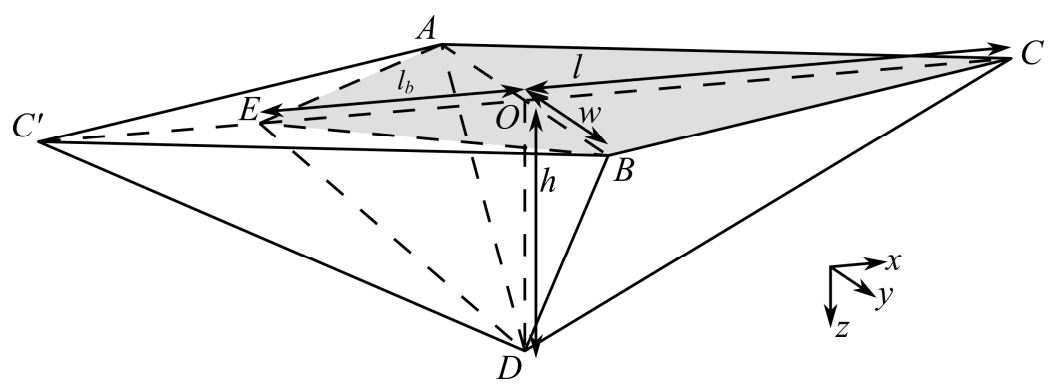

Figure 4.11. Tip geometry with the extended base $A C^{\prime} B C$. The grey quadrilateral $A E B C$ is the region that (mainly) carries the plastic forces.

One of the assumptions of the model is that the asperity is a rigid body. If that assumption is used, the equations (4.8) and (4.9) remain valid, by calculating the values of $\bar{x}_{c}, \bar{A}_{A B C}$ and $\bar{I}_{c y y}$ for $A C^{\prime} B C$ instead of $A B C$. These values can be calculated using equations (A.24) to (A.26). The result of this extended base is shown in figure 4.12. From the graphs it becomes clear that the ratio $\sigma_{A B C} / p_{p l}$ gets a value in the order of -1 for a wide range of $\bar{h}$. That means that at values of $\bar{h}$ of about 1 , using $A C^{\prime} B C$ as the base of the asperity, the stresses are much lower than in the case where $A B C$ is used as base. But for values of $\bar{h}$, let say $\bar{h}<0.2, A B C$ will give better results than $A C^{\prime} B C$ as the asperity base. The reason is, that in the case of small values of $\bar{h}$ the stress state should be close to the contact stress, so $\sigma_{A B C} / p_{p l}$ should be close to -1 . Using $A C^{\prime} B C$ results in a higher compressive stress, namely $\sigma_{A B C} / p_{p l} \approx-1.5$ for $\bar{h}<<1$. This stress is the result of the lever effect of the extended rigid asperity. This lever effect is not realistic in the case of low values of $\bar{h}$. A low value of $\bar{h}$ does not give enough stiffness to justify the assumption of a rigid asperity. So, a symmetrical pyramid, with the $y z$-plane as its symmetry plane, cannot be used immediately. 


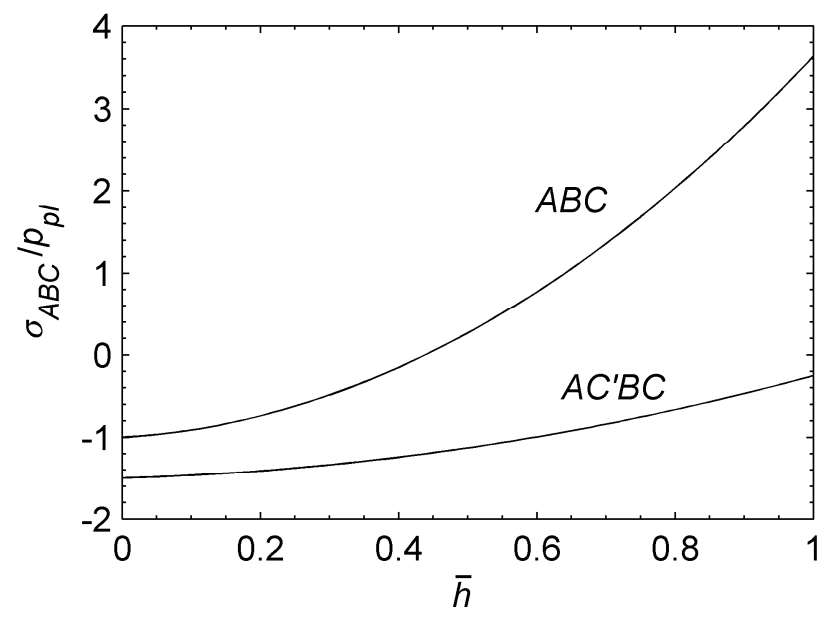

Figure 4.12. Normal stress over plastic pressure as a function of normalized asperity height on faces $A B C$ and $A C^{\prime} B C$. The conditions are: $\bar{x}=1$ for $\bar{w}=1$ and $\mu=0.1$.

From figure 4.12 it can be concluded that the part of the base that carries the load is influencing the stresses significantly. The area that carries the load is not known beforehand. Therefore FEM calculations will be performed in order to estimate the load carrying area.

Because of the finite stiffness of the asperity, the plastic load on it is carried by the material near the loaded area. To get more details about the loading area, as mentioned before, FEM-analyses have been performed on the asperity as shown in figure 4.11. The analyses have the following properties:

- The asperity is built from elastic tetrahedral elements.

- For comparison of the support of the asperity, calculations are performed for both a rigid and an elastic support of the asperity. The elastic support is made from tetrahedral elements, in fact a part of the elastic bulk material is modelled.

- The faces $B D C$ and $A C D$ are loaded with a unit normal pressure that represents the plastic normal load. The plastic shear stress gets the value $\mu$. This way of loading corresponds to normalizing the plastic stresses by $p_{p l}$.

- The calculations are performed for different values of $\bar{w}$ and $\bar{h}$.

Some FEM results are presented in a number of figures. In figures 4.13 to 4.15 all normalized stress components are presented for one asperity geometry. Only these results are shown, because the results for these situations with $\bar{h}<0.3$ and $0.5<\bar{w}<2$ give 
comparable results. Most attention will be paid to $\sigma_{x z}$, see figure $4.15 \mathrm{a}$, because these results will be used later on for formulating a failure criterion, see figure 4.16.
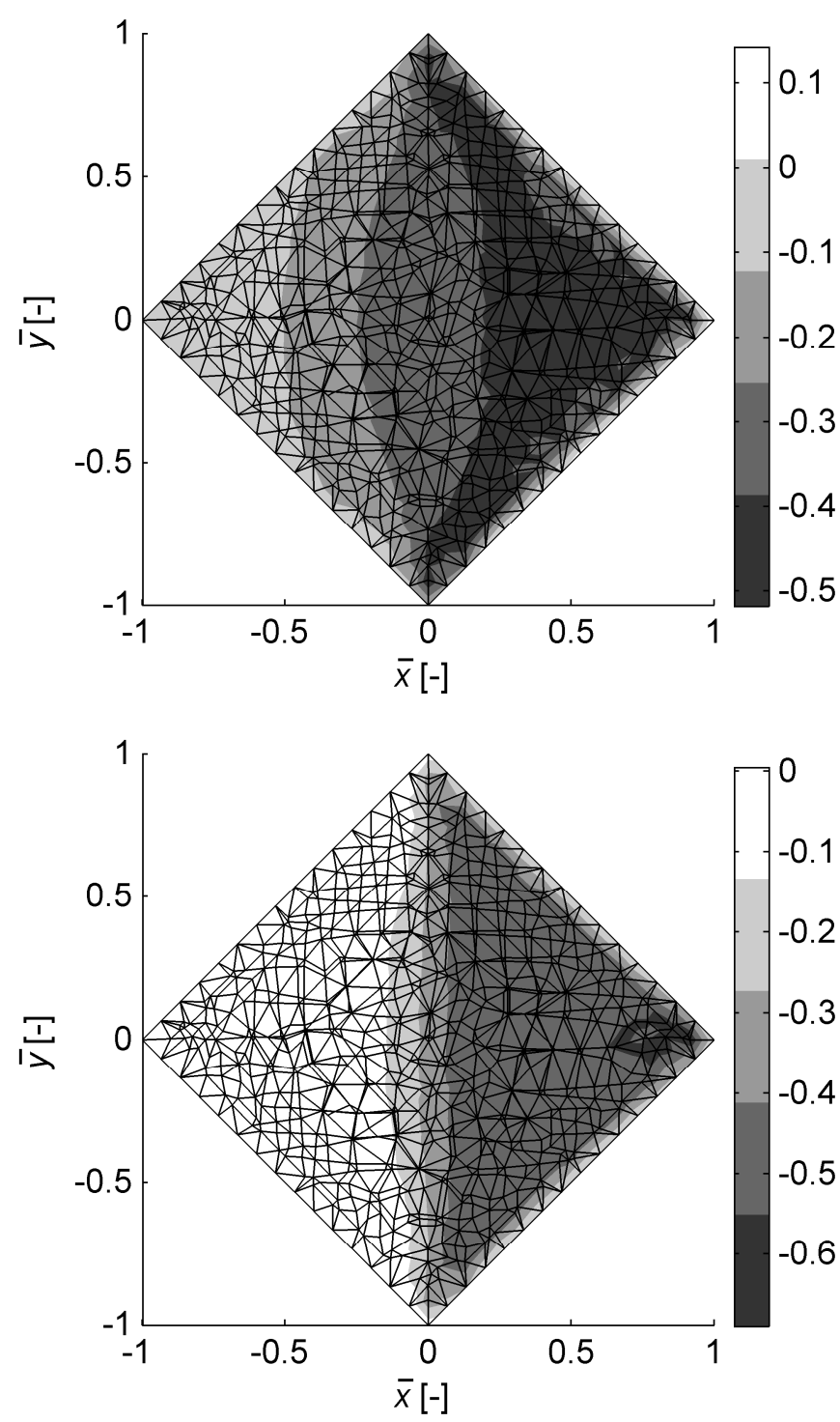

$b$

Figure 4.13. Normalized stresses on plane $A C^{\prime} B C$ of pyramidal asperity. Given are $\sigma_{x x} / p_{p l}$ (a) and $\sigma_{y y} / p_{p l}$ (b). The conditions are: $\bar{w}=1, \bar{h}=0.2$ and $\mu=0.1$. 

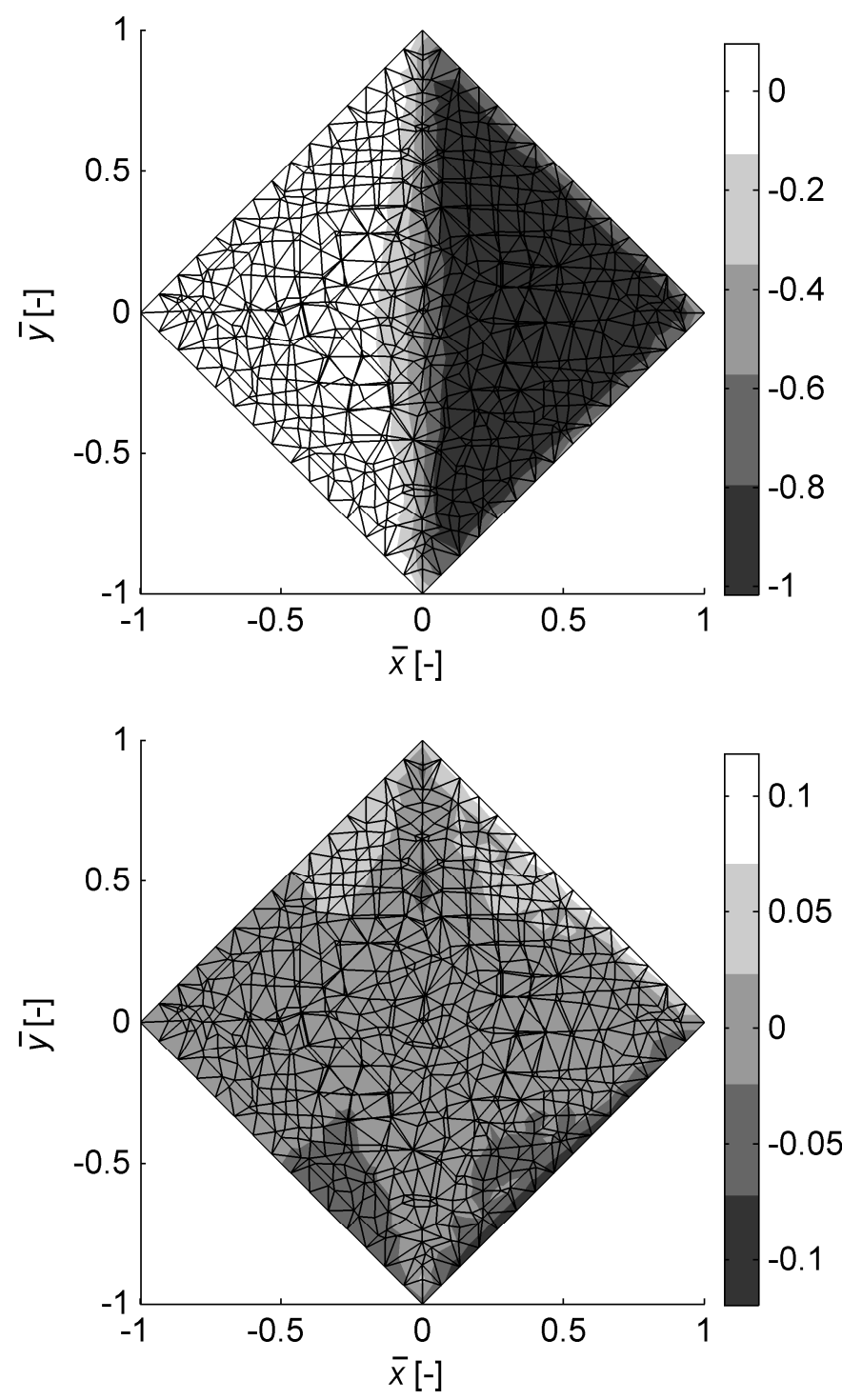

b

Figure 4.14. Normalized stresses on plane $A C^{\prime} B C$ of pyramidal asperity. Given are $\sigma_{z z} / p_{p l}$ (a) and $\sigma_{x y} / p_{p l}(b)$. The conditions are: $\bar{w}=1, \bar{h}=0.2$ and $\mu=0.1$. 


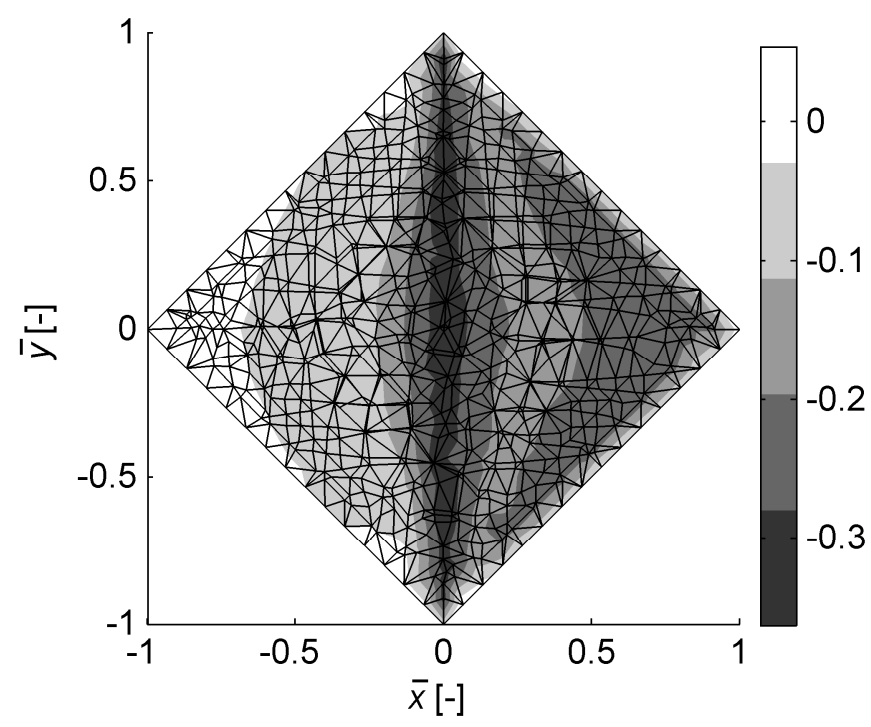

$a$

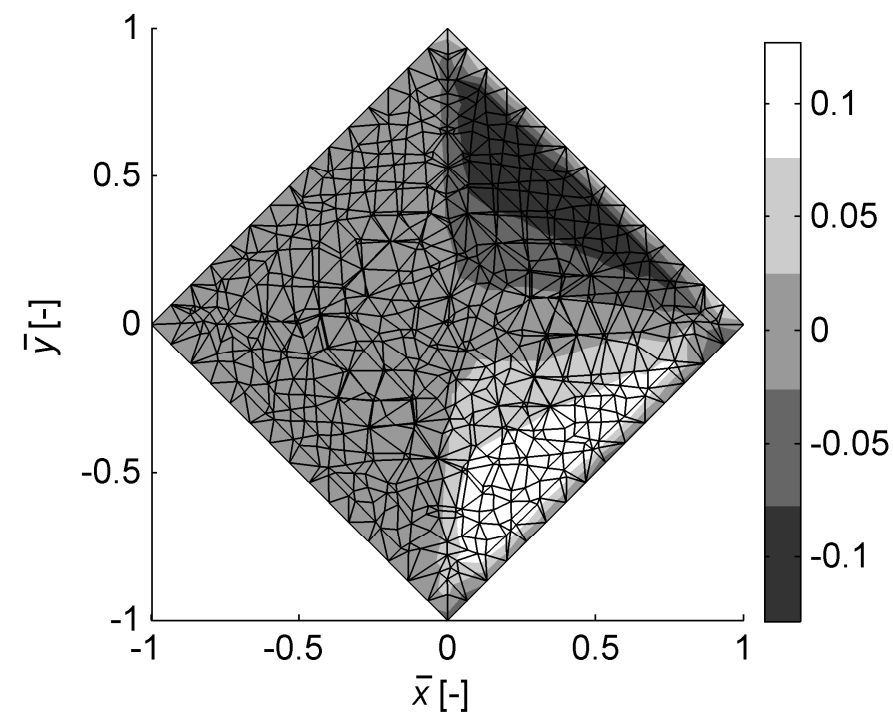

b

Figure 4.15. Normalized stresses on plane $A C^{\prime} B C$ of pyramidal asperity. Given are $\sigma_{x z} / p_{p l}$ (a) and $\sigma_{y z} / p_{p l}(b)$. The conditions are: $\bar{w}=1, \bar{h}=0.2$ and $\mu=0.1$. 


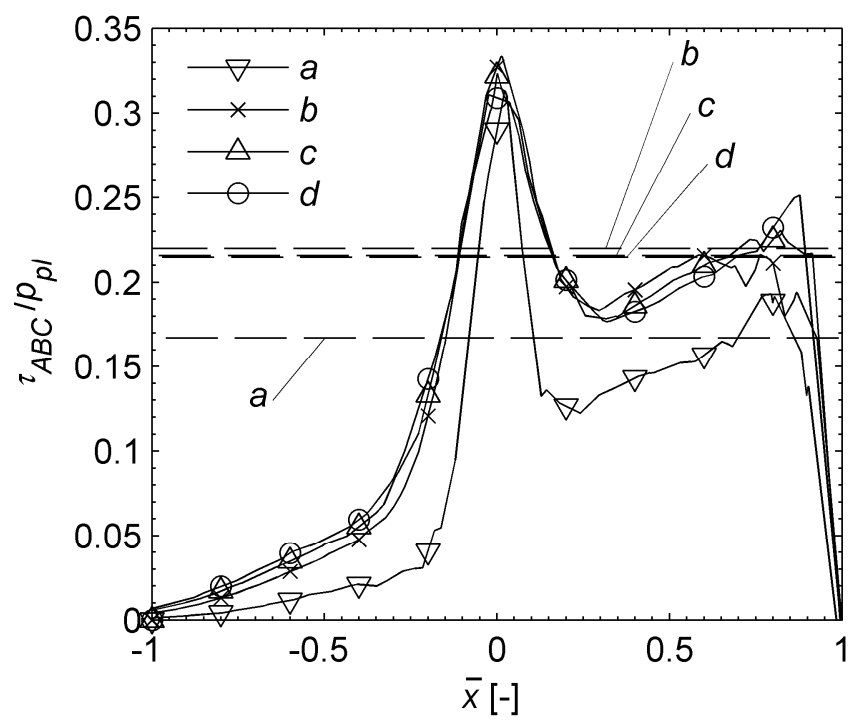

Figure 4.16. Shear stress over plastic pressure along the $x$-axis on face $A C^{\prime} B C$. Comparison of model (dashed lines) and FEM calculations (lines with markers). The sign of the FEM calculations is changed in the plot, in fact, in the plot it is given as $\left(-\sigma_{x z} / p_{p \nu}\right)$. The presented situations are $\bar{h}=0.1, \bar{w}=1(a) ; \bar{h}=0.2, \bar{w}=0.5(b) ; \bar{h}=0.2, \bar{w}=1$ (c) and $\bar{h}$ $=0.2, \bar{w}=2(b)$.

From these FEM-analyses it follows that the area that actually carries the load is a function of $\bar{h}$, as can be seen in figure 4.16. The stresses in the figure given for the three asperities with the same height $(\bar{h}=0.2)$ distribute the load in almost the same manner over the face AC'BC. The lower asperity $(\bar{h}=0.1)$ has a lower shear stress on this face and is distributed less in backwards direction. From these calculations it is concluded that the area on the $x y$-plane that carries (mainly) the load is the quadrilateral $A E B C$, see figure 4.11. Vertex $E$ has coordinates $\left(-l_{b}, 0,0\right)$. The value of $l_{b}$ as a function of $h$ can be approximated by $l_{b}=c h$, where $c$ is a constant that has a value of about 2. The stress level is low beyond $\bar{x}<c \bar{h}$ and the shear stress $\tau_{A B C}$ is approximated well by equation (4.8), as is shown by the dashed lines in figure 4.16. The value of $c$ has been obtained from FEM simulations where $\bar{h}<0.3$. The value of $l_{b}$ has the geometrical restriction that $E$ remains within the base $A C^{\prime} B C$. For approximating the internal stresses in the asperity, the focus is fully on the pyramid $A E B C D$. The role of $A C^{\prime} B E D$ is negligible. From the FEM-analyses it follows that equation (4.9) is not a good estimator for the normal stresses in $z$-direction. The normal stress in $z$-direction is not a linear function of $x$, as given by equation (4.9), but nearly constant in the 
loaded area equal to $p_{p l}$, see figure 4.14a. Near the $y z$-plane the normal stress in $z$-direction decreases, because plastic load acts only at the side of the positive $x$-axis of the $y z$-plane, but the load can be carried at both sides of this plane.

\subsubsection{Asperity fail criterion}

To define a fail criterion, stress relations can be used such as the relations of Von Mises or Tresca. These relations need the complete stress state. The stress components $\sigma_{z z}$ and $\tau_{x z}$ are relatively easy to obtain as mentioned above. According to the FEM analyses the values of $\sigma_{x x}$ and $\sigma_{y y}$ are about a half of the value of $\sigma_{z z}$. Shear stress $\tau_{x y}$ has a relatively small value, about a tenth of $\tau_{x z}, \tau_{y z}$ has values in the order of $\tau_{x z}$. Both, $\tau_{x y}$ and $\tau_{y z}$ are zero on the $x z$-plane due to symmetry. Even though four of the six components are only described qualitatively, a stress criterion can still be given. If a plastic failure is assumed, plastic deformation can be described using the relation of Prandl-Reuss [9]. This is given for the situation that the Von Mises stress exceeds $\sigma_{y}$. In index notation, this relation is given as:

$$
d \varepsilon_{i j}=\frac{1}{E}\left[(1+v) d \sigma_{i j}-v \delta_{i j} d \sigma_{k k}\right]+\left(\sigma_{i j}-\frac{1}{3} \sigma_{k k} \delta_{i j}\right) d \lambda
$$

In equation (4.14) the deviation of strain is given as a function of the deviation of stresses (elastic effect) and the stresses itself (plastic effect). In this relation $E$, is Young's modulus, $v$ is Poisson's ratio and $\delta$ is the Kronecker delta. The term $d \lambda$ relates the plastic deformation velocity to the stresses and is only used here as a constant without further explanation. According to equation (4.14) shear strain with indices $i j$ is only related to other tensor components with indices $i j$, so independent of tensor components with different indices. That makes the three shear strain equations independent relations. The normal strains are coupled by the effect of the hydrostatic pressure, as given by the terms with $\delta_{i j}$.

To formulate the normal strain relations, the following is assumed:

- The material in the surface is fixed in $x$ and $y$-direction by its surrounded material. Therefore: $d \varepsilon_{11}=d \varepsilon_{22}=0$. In $z$-direction the material can be pressed into the contact.

- The load in $z$-direction is constant: $\sigma_{33}=p_{p l}$. Therefore: $d \sigma_{33}=0$.

(To remain close to the index notation as given in equation (4.14), the indices $x, y$ and $z$ are temporarily replaced by the 1,2 and 3.)

Now, the following set of equations can be derived:

$$
\left\{\begin{array}{l}
d \sigma_{11} \\
d \sigma_{22}
\end{array}\right\}=-\frac{E d \lambda}{3\left(1-v^{2}\right)}\left[\begin{array}{ll}
1 & v \\
v & 1
\end{array}\right]\left(\left[\begin{array}{cc}
2 & -1 \\
-1 & 2
\end{array}\right]\left\{\begin{array}{l}
\sigma_{11} \\
\sigma_{22}
\end{array}\right\}+\left\{\begin{array}{l}
p_{p l} \\
p_{p l}
\end{array}\right\}\right)
$$


The amplitude of equation (4.15) is not known, because of the unknown $d \lambda$, but still the direction of the deformation can be determined, as is given in the vector plot of figure 4.17. In this figure only the stresses are given that are in the range $-1<\sigma / p_{p l}<0$, because the stresses are according to the FEM calculation in this range before plastic deformation. According to figure 4.17, the deformation goes in the direction of the pure hydrostatic pressure situation. The strain before the hydrostatic pressure situation is reached is very small. In the case of the hydrostatic pressure only, the stresses are fully elastic. Contact pressures are in the order of $1 \mathrm{GPa}$ (a typical hardness value), $E$ is in the order of $100 \mathrm{GPa}$, so the hydrostatic situation is already reached after a deformation in the order of one percent.

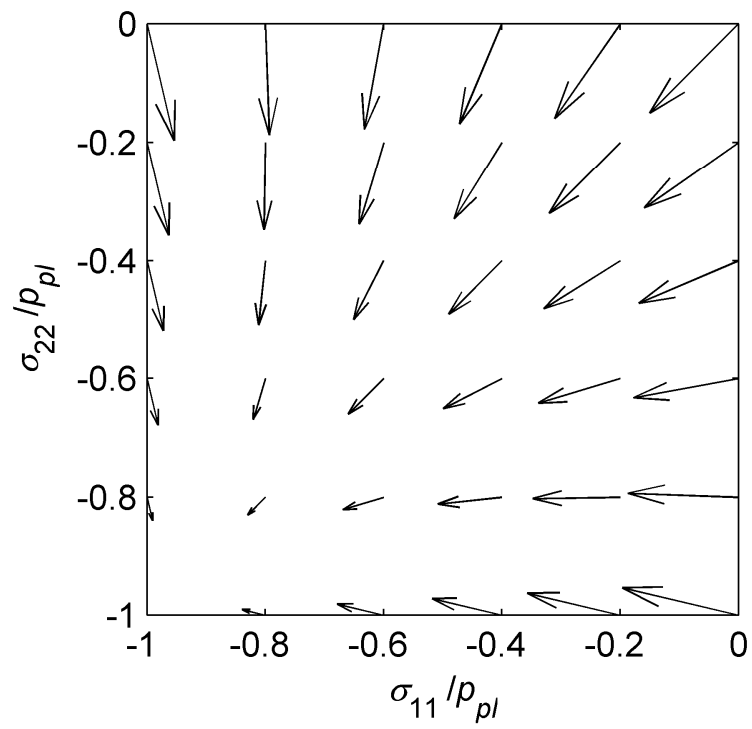

Figure 4.17. Vector plot of the stress deviation as a function of the stresses in $x$ and $y$-direction in the case of plastic deformation in the contact zone of an asperity.

The next step is the effect of the shear strain components. According to equation (4.14), a strain component $\varepsilon_{i j}$, with $i \neq j$, is only a function of the stress components $\sigma_{i j}$ and $d \sigma_{i j}$ and is not coupled to stresses with other indices. So, the equations for the shear strain components form a set of uncoupled equations and the effect of every separate shear stress component can be discussed on its own, as will be done below.

- The deformation due to $\sigma_{12}$ is relatively small, because its value is much smaller than the other components, so its relevance is limited. 
- The deformation due to $\sigma_{13}$ is of importance, because its value is not small related to the other stresses and there is no stabilization after plastic deformation, because shearing in backwards direction of the asperity does not give a fundamental change in the load situation.

- In the case of $\sigma_{23}$ the stress is negative for positive $y$-coordinates and positive for negative $y$-coordinates. That means that the material is compressed, so this stress causes a stabilizing action.

From the analysis as described above it follows that most stress components generate a plastic deformation into the direction of a more stable situation. This stabilization is already realized after small deformations. This statement does not hold clearly for $\sigma_{13}$. Therefore, this stress will be used as the failure criterion for an asperity or a lump on an asperity. When according to this stress component the asperity deforms plastically, it is stated that it fails. This stress will be calculated using equation (4.8).

\subsubsection{Crack formation}

The asperity formulated in this chapter has an idealized geometry. In practice an asperity does not have a well defined shape and may contain a crack. An irregular shape can be approximated by choosing the proper dimensions $w$ and $l$. To deal with a crack in the asperity, the model will be adapted.

The crack is supposed to be in the face $A B C$ and starts at vertex $C$ and has length $l_{c r}$, see figure 4.18. If the crack is situated in the face $A B C$, it is possible to more or less use the same strategy as in section 4.3.4. The plastic forces on the faces $B D C$ and $A C D$ are not changed, but only the internal bearing area of face $A B C$ (or $A E B C$ ). Changing this bearing area changes some important quantities. First, the bearing area $\bar{A}_{A B C}$ itself reduces and consequently $\bar{I}_{c y y}$ too. These reduced quantities immediately have effect on the stresses according to equations (4.8) and (4.9). But, as already discussed in section 4.3.4, the stress ratio $\sigma_{A B C} / p_{p l}$ is about -1 . That means a compressive stress state, so $\bar{A}_{A B C}$ and $\bar{I}_{c y y}$ do not reduce effectively and $\sigma_{A B C} / p_{p l}$ do not change due to the crack. In the case of $\tau_{A B C} / p_{p l}$ the effect of the crack depends on the surface behaviour of the crack. The extremes are full adhesion of the crack surfaces and no interaction in the $x$-direction. In the case of full interaction, there is no crack anymore and for $\tau_{A B C} / p_{p l}$ equation (4.8) remains unchanged. In the case of no interaction in the $x$-direction, the value of $\bar{A}_{A B C}$ in equation (4.8) has to be reduced by the dimensionless size of the crack area $\bar{w} \bar{l}_{c r}{ }^{2}$. That results in an inversely proportional relation between $\tau_{A B C} / p_{p l}$ and the value of the remaining area $\bar{A}_{A B C}$, which is worked out in section 4.3.7. 


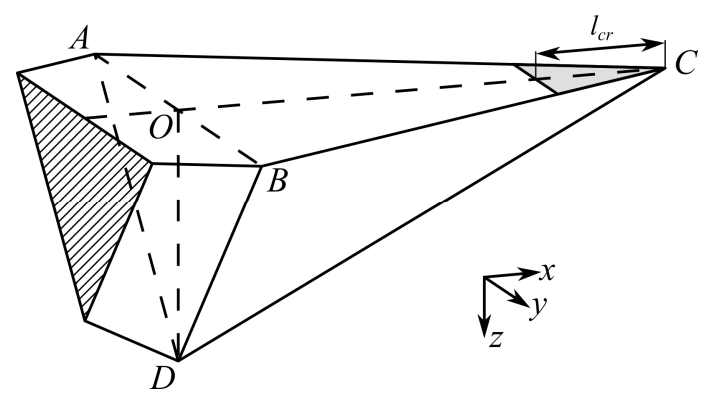

Figure 4.18. Part of tip geometry with crack at vertex $C$ (grey area).

\subsubsection{Lump failure model}

A lump failure criterion is formulated on the basis of the statics analyses as presented in the section 4.3.2 and appendix A. In sections 4.3.4 and 4.3.5 the importance of equation (4.8) is discussed and it is concluded that this equation gives a good estimator when an asperity fails and therefore will be used as a fail criterion. The implementation of this criterion will be discussed in this section.

As a basis of the implementation, equation (4.8) will be used. This equation itself gives only the shear stress in $x$-direction on the face $A B C$. In sections 4.3.4 and 4.3.6 some adaptations are already presented: adaptation of the asperity geometry and the effect of crack formation. These adaptations play with area $\bar{A}_{A B C}$ over which the force in $x$-direction has to be spread. The next step is to implement the strength of the material in the asperity and of the interface between the asperity and plastic deforming material. Finally a stability criterion has to be formulated under which circumstances an asperity can exist.

The criterion equation (4.8) will be formulated in a different way and some new variables are defined. In sections 4.3.4 and 4.3.6 the variable $\bar{A}_{A B C}$ is already interpreted in a more flexible way than its name suggests. According to its name, it is the size of the triangle $A B C$. The area size of triangle $A B C$ is $w l$, or $\bar{w}$ in dimensionless form. The variable $\bar{A}_{A B C}$ will be substituted by:

$$
\bar{A}_{A B C}=\left(1+c \bar{h}-\bar{l}_{c r}^{2}\right) \bar{w}
$$

Here, $\bar{A}_{A B C}$ is corrected for a changing base of the asperity. The correction factor $c$ is the factor as defined in section 4.3.4, $\bar{l}_{c r}$ is the dimensionless length of the crack as defined in section 4.3.6.

Using equation (4.16) in equation (4.8) and introducing the dimensionless shear strength $T$, defined as the shear strength divided by $p_{p l}$, the following inequality can be constructed: 


$$
T \geq \frac{1}{1+c \bar{h}-\bar{l}_{c r}^{2}}\left(\bar{h}+\mu \sqrt{1+(\bar{h} / \bar{w})^{2}}\right)
$$

The structure of inequality (4.17) is derived from the plastic force divided by a bearing area. Rewriting this inequality to a form that gives a more direct insight in the dependency of $\bar{h}$ gives the following:

$$
\left(1-\bar{l}_{c r}{ }^{2}\right) T+(c T-1) \bar{h} \geq \mu \sqrt{1+(\bar{h} / \bar{w})^{2}}
$$

The formulation of inequality (4.18) gives at the left-hand side the force that can be carried at the adapted area of $\bar{A}_{A B C}$ minus the force due to the plastic normal pressure. At the right-hand side the force remains due to the plastic shear stress at the interface between the tip and plastic deforming material. Reformulating (4.17) to (4.18) gives a linear function of $\bar{h}$ crossing a square root function of $\bar{h}$. This inequality gives in a more orderly fashion the effect of $c, \bar{l}_{c r}$ and $\mu$. The solution for this inequality will be given now.

Treating (4.18) as an equality, it can have at most two solutions, called here $\bar{h}_{\min }$ and $\bar{h}_{\max }$ These solutions are:

$$
\begin{aligned}
& \bar{h}_{\min }=\frac{T(1-c T)\left(1-\bar{l}_{c r}{ }^{2}\right)+\mu \sqrt{T^{2}\left(1-\bar{l}_{c r}{ }^{2}\right)^{2} / \bar{w}^{2}+(1-c T)^{2}-\mu^{2} / \bar{w}^{2}}}{(1-c T)^{2}-\mu^{2} / \bar{w}^{2}} \\
& \bar{h}_{\max }=\frac{T(1-c T)\left(1-\bar{l}_{c r}{ }^{2}\right)-\mu \sqrt{T^{2}\left(1-\bar{l}_{c r}{ }^{2}\right)^{2} / \bar{w}^{2}+(1-c T)^{2}-\mu^{2} / \bar{w}^{2}}}{(1-c T)^{2}-\mu^{2} / \bar{w}^{2}}
\end{aligned}
$$

And the limit situation for $|1-c T|=\mu / \bar{w}$ :

$$
\bar{h}_{\max }=\frac{\bar{w}}{2}\left|\frac{\left(1-\bar{l}_{c r}{ }^{2}\right) T}{\mu}-\frac{\mu}{\left(1-\bar{l}_{c r}{ }^{2}\right) T}\right|
$$

The possible solutions of inequality (4.18) in a specific case are given in tables $4.1 \mathrm{a}$ and $4.1 \mathrm{~b}$. 


\begin{tabular}{|l|l|l|l|}
\cline { 2 - 4 } \multicolumn{1}{c|}{} & $c T<1$ & $c T=1$ & $c T>1$ \\
\hline$\mu=0$ & $\bar{h} \leq \bar{h}_{\max }$ & no maximum of $\bar{h}$ & no maximum of $\bar{h}$ \\
\hline$\mu<\left(1-\bar{l}_{c r}{ }^{2}\right) T$ & $\bar{h} \leq \bar{h}_{\max }$ & $\bar{h} \leq \bar{h}_{\max }$ & see table 4.1b \\
\hline$\mu \geq\left(1-\bar{l}_{c r}{ }^{2}\right) T$ & $\bar{h}=0$ & $\bar{h}=0$ & see table 4.1b \\
\hline
\end{tabular}

Table 4.1a. Solutions of inequality (4.18) for different situations.

\begin{tabular}{|l|l|l|}
\cline { 2 - 3 } \multicolumn{1}{c|}{} & $\mu<\left(1-\bar{l}_{c r}^{2}\right) T$ & $\mu \geq\left(1-\bar{l}_{c r}^{2}\right) T$ \\
\hline$\mu / \bar{w} \leq c T-1$ & no maximum of $\bar{h}$ & $\bar{h} \geq \bar{h}_{\min }$ \\
\hline$(c T-1)^{2}<\frac{\mu^{2}}{\bar{w}^{2}} \leq(c T-1)^{2}+\frac{\left(1-\bar{l}_{c r}^{2}\right)^{2} T^{2}}{\bar{w}^{2}}$ & $\bar{h} \leq \bar{h}_{\max }$ & $\bar{h}_{\min } \leq \bar{h} \leq \bar{h}_{\max }$ \\
\hline$\frac{\mu^{2}}{\bar{w}^{2}}>(c T-1)^{2}+\frac{\left(1-\bar{l}_{c r}^{2}\right)^{2} T^{2}}{\bar{w}^{2}}$ & $\bar{h} \leq \bar{h}_{\max }$ & $\bar{h}=0$ \\
\hline
\end{tabular}

Table 4.1b. Solutions of inequality (4.18), detail of table 4.1a for cT $>1$ and $\mu>0$.

For the situation that $\bar{l}_{b}\left(\bar{l}_{b}=c \bar{h}\right)$ exceeds the value of 1 , equation (4.16) is not valid anymore, because $\bar{A}_{A B C}$ is limited by the area of $A C^{\prime} B C$. This results in a limitation of $\bar{h}$ to:

$$
\bar{h} \leq \frac{T\left(2-\bar{l}_{c r}{ }^{2}\right)-\mu \sqrt{T^{2}\left(2-\bar{l}_{c r}{ }^{2}\right)^{2} / \bar{w}^{2}+1-\mu^{2} / \bar{w}^{2}}}{1-\mu^{2} / \bar{w}^{2}}
$$

As long as the inequalities (4.18) and (4.20) are met, the asperity will resist the plastic forces, otherwise it fails. This gives a criterion of the maximum possible value of $\bar{h}$. The value of $\bar{h}$ is according to its definition the tangent of the line $C D$ with the $x$-axis, so by equation (4.18) the maximum attack angle of an asperity is given. In the figures 4.19 and 4.20 a number of results are shown.

From the figures 4.19 and 4.20 the effect of the different variables is presented. The effect of $\mu$ becomes clear in all graphs. An increasing value of $\mu$ results in an increasing force on the tip, so the maximum tip height reduces. The effect of lubricant, contamination or other situations that influence the interface strength has a clear effect on the maximum height. 


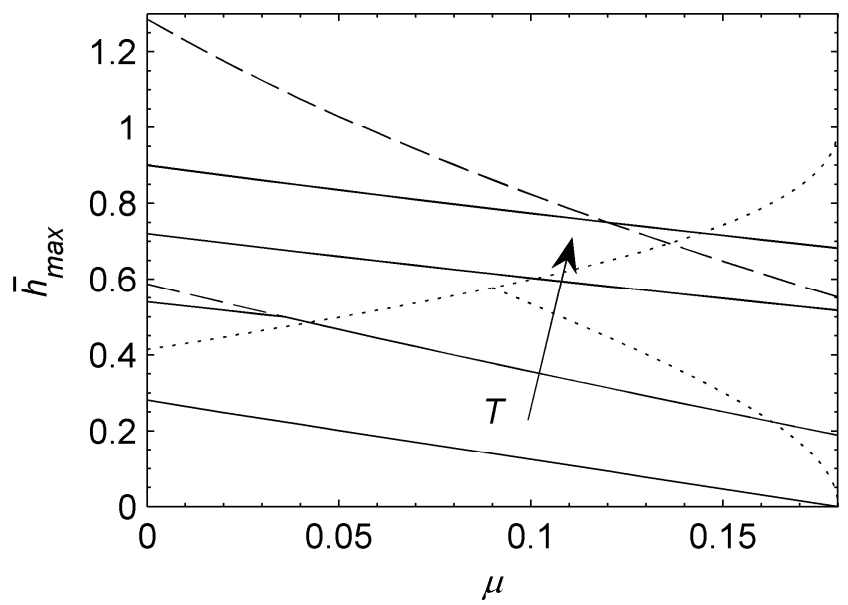

a

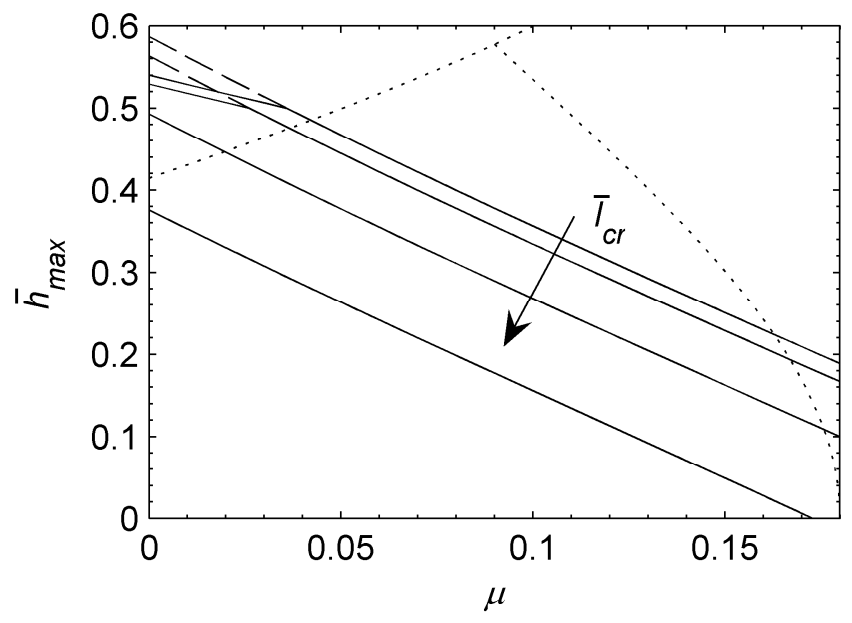

b

Figure 4.19. Maximum possible values of $\bar{h}$ as a function of the plastic coefficient of friction for different properties of the asperity. The default values are $T=0.27, \bar{l}_{c r}=0, c=2$ and $\bar{w}=1$. The varied variables increase in the direction of the arrows. The dashed lines show the graphs in the case where the constraint of inequality (4.20) was not used. The variations are: (a) $T=0.18,0.27,0.36$ and 0.45. The 'free' dashed line belongs to $T=0.36$ and the dashed line of $T=0.45$ is omitted, because it lies far above the other graphs. (b) $\bar{l}_{c r}=0,0.2,0.4$ and 0.6 .

The dotted lines show the transitions between different wear regimes as described in section 2.2.3 and as shown in figure 2.10. 
The effect of $T$ is another quantity that has a strong effect on the maximum tip height. From table 4.1a it follows that in the case where the tip material has the same strength as the plastic material through which it ploughs, so $T=0.18$ according to equation (4.11), the tip cannot grow in the case of $\mu=0.18$. This is obvious, because this situation corresponds with the situation within the bulk of the plastic material. In case where $c T \geq 1$ and $\mu=0, \bar{h}$ has no maximum, according to table 4.1a. If inequality (4.20) is taken into account, $\bar{h}$ always has a maximum. The effect of inequality (4.20) is visible in the figures 4.19 and 4.20 as the difference in the dashed and solid graphs. From the graphs in figure 4.19a the strong effect of $\mathrm{T}$ becomes clear, but the effect is weakened by inequality (4.20).

The effect of the crack size is shown in figure $4.19 \mathrm{~b}$. In the given situation, the effect of $\bar{l}_{c r}$ on the maximum value of $\bar{h}$ which decreases by an increasing value of $\bar{l}_{c r}$. This can be explained by the fact that the crack area size is the square of $\bar{l}_{c r}$. There is almost a linear relation between the crack area size and the maximum tip height. Some elucidation is required about using $\bar{l}_{c r}$. In order to study the sensitivity of a crack, it is shown in the formula given in this section as well as in the graphs of figure $4.19 \mathrm{~b}$. However, using it in a practical way is more difficult. When starting from real surface roughness data, which have been obtained through optical techniques, it only contains surface data and not the cracks below the surface. An additional second problem is the state of the crack. If it is a dry crack, in which case there is still a lot of adhesion, then $\bar{l}_{c r}$ gives an overestimation of the effect of the crack, because both crack surfaces are still interacting. However, if the crack is lubricated or otherwise contaminated and low adhesion consequently exists, then $\bar{l}_{c r}$ gives a good estimation of the reduced strength. A third problem is the changeability of $\bar{l}_{c r}$. The value of $\bar{l}_{c r}$ will increase due to a growing crack. But as the tip grows through material transfer the value of $\bar{l}_{c r}$ may decrease, because the absolute crack size remains the same and the tip grows.

Figure $4.20 \mathrm{a}$ presents the effect of the factor $c$. This factor will not, in fact, be varied, because it is a constant determined by FEM-calculations. The said determination of $c$ is an estimation, in the sense that the model calculated a shear stress $\tau_{A B C}$ that is close to the values of the FEM-calculation. The figure shows the sensitivity of this estimation. As long as inequality (4.20) has no effect, the factor $c$ has a clear effect. However, in the case where inequality (4.20) restricts $\bar{h}$, it can be seen that $c$ no longer has an effect. This is obvious and was taken into account as the reason why inequality (4.20) is introduced. 


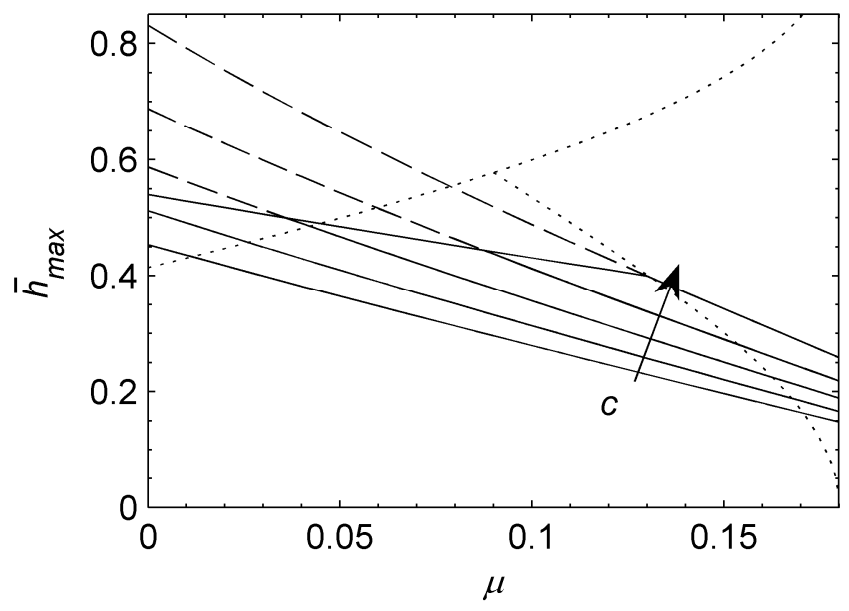

a

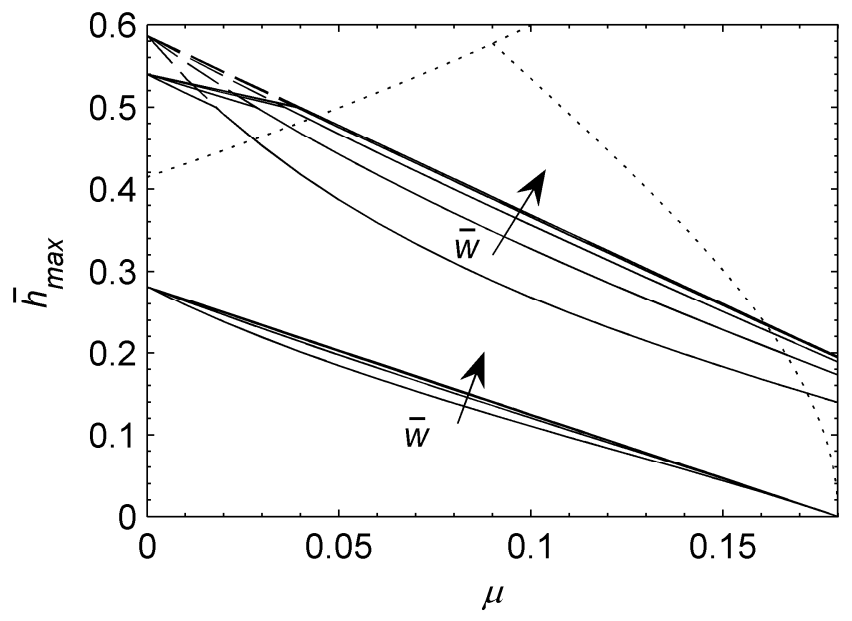

b

Figure 4.20. Maximum possible values of $\bar{h}$ as a function of the plastic coefficient of friction for different properties of the asperity. The default values are $T=0.27, \bar{l}_{c r}=0, c=2$ and $\bar{w}=1$. The varied variables increase in the direction of the arrows. The dashed lines show the graphs in the case where the constraint of inequality (4.20) was not used. The variations are: (a) $c=1.5,1.75,2,2.25$ and 2.5. (b) $\bar{w}=0.25,0.5,1,2$ and 4. The lower set of lines is for $T=0.18$, the higher set for $T=0.27$. Due to the close results a number of lines have a huge overlap.

The dotted lines show the boundaries of the different wear regimes, see description of figure 4.19 . 
The width $\bar{w}$ of the asperity has a weak effect on the maximum value of $\bar{h}$. In figure $4.20 \mathrm{~b}$ a large range of $\bar{w}$ is presented. Only for small values of $\bar{w}, \bar{w}<1$, the effect of $\bar{w}$ is clearly visible. In inequality (4.18) $\bar{w}$ is enclosed in the term $\bar{h} / \bar{w}$. The effect of this term is shown in figure $4.20 \mathrm{~b}$ by the sets of graphs for different values of $T$. A higher value of $T$ results in a higher value of $\bar{h}$ and so in a stronger effect of $\bar{w}$. For even higher values of $T$ as presented in figure $4.20 \mathrm{~b}$, the effect of $\bar{w}$ would be stronger according to inequality (4.18), but is mostly restricted by the constraint of inequality (4.20).

From the graphs and the analysis it can be concluded that the $\mu$ and $T$ are the most important variables for using the model. The effect of cracks is difficult to implement and $\bar{w}$ can be implemented easily, but has no strong effect. Obtaining proper values of $\mu$ and $T$ is the most challenging task in using the model.

Figures 4.19 and 4.20 show lines (dotted lines) that give the transition between different wear regimes as described in section 2.2.3. According to [39] the material transfer from the workpiece to the tool occurs in the wedge regime. Section 4.4.3 explains why it is likely that transfer occurs in this regime. To show the area of the wedge regime on a $(\mu, \bar{h}$ )-coordinate system, the diagram as shown in figure 2.10 has to be transformed from the $\left(f_{H K}, \theta\right)$-coordinate system. Using the definition of $\mu$ and equation (4.11), it follows that $\mu=0.18 f_{H K}$. Both, $\theta$ and $\bar{h}$ give a (kind of) attack angle and are related as $\bar{h}=\tan (\theta)$. Using these relations of $\mu$ and $\bar{h}$ the wear mode diagram can be given in the $(\mu, \bar{h})$-coordinate system.

Combining the results of the maximum possible values of $\bar{h}$ and the wear mode diagram, some conclusions can be drawn. When lump growth only happens in the wedge regime, $\mu>0.09$ and the value of $\bar{h}$ cannot exceed 1 due to lump growth. For values of $\mu$ slightly more than 0.09 , lump growth takes place in a very narrow band of $\bar{h}$-values. For values of $\mu$ of about 0.18 , lump growth can occur in a large range of $\bar{h}$ and for $\mu=0.18$ for $\bar{h}<1$ (so, practically for every asperity) lump growth will occur. Thus, a higher value of $\mu$ gives more risk of galling.

\subsection{Adhesion}

In order to adhere to the tool surface, the adhesion force between the sheet material and the tool material needs to be sufficiently high. Adhesion is the attraction between dissimilar atoms or molecules. By contrast, cohesion takes place between similar atoms or molecules. Here, only adhesion is taken into account and cohesion will be seen as a special form of adhesion, where both interacting atoms or molecules are the same. Because the research deals with metals, atoms will be spoken about here.

Adhesion originates from different mechanisms. The mechanisms are: 
- Mechanical interlocking. Two rough surfaces stick or hook together due to surface irregularities.

- Diffusion: Atoms diffuse across the interface between two contacting bodies, for example compatible metals as in galvannealing, a diffusion process that is used for zinc on steel.

- Electrostatic: Electrostatic forces act between surfaces. This electrostatic situation can be originated from electron transfer, like in tribo charging.

- Chemical bond or chemisorption: Strong chemical bonds are formed on the interface, like metallic, ionic or covalent bonds.

- Van der Waals attraction: Intermolecular forces or meniscus forces.

In this section, the focus is on Van der Waals attraction and on metallic bonds. In the metal contact, on the short distance, lower than $0.5 \mathrm{~nm}$, metallic bonds are dominating, due to electron exchange interactions [26]. For larger separations, the Van der Waals forces are dominating.

In section 4.4.1, the importance of geometry aspects compared to material aspects is presented. Here, it is explained why material aspects are of more importance than the geometrical aspects, as long as bodies that attract each other have a certain size. In section 4.4.2 the work of adhesion is further discussed, based on interfacial and surface energies. In section 4.4.3 the adhesion theory is compared with the situation in practice.

\subsubsection{Geometry and material aspects of adhesion}

An important aspect of the adhesion theory is that the interaction energy can be separated into a geometry dependent part and a geometry independent part. The impact of these parts is presented in this section. These effects are studied on the basis of interaction between two atoms. By pairwise addition this is extended to the adhesion interaction between two bodies.

The interaction energy between two individual atoms $a$ and $b$ is given by $-C_{a b} / d^{6}$. Here, $C_{a b}$ gives the interaction energy at a unit distance, dependent on the types of atoms $a$ and $b$ and $d$ the distance between the atoms. In this relation, only the attracting term is taken into account, because the repulsive term decreases very strongly as a function of $d$, as given in the Lennard-Jones potential, being proportional to $1 / d^{12}$. By pairwise additivity of all interacting atoms in two bodies, the interaction energy between two bodies can be calculated. This calculation is given in appendix B. In this appendix it follows from the equations (B.3) and (B.4) that the interactive energy can be separated into a geometry dependent part and a geometry independent part. The terms before the integrals are geometry independent and are proportional to the Hamaker constant. The integrals are taken over the body geometries and so geometry dependent. 
The Hamaker constant is defined as $A_{H}=\pi^{2} C_{a b} \rho_{a} \rho_{b}$, where $\rho_{a}$ and $\rho_{b}$ are the number of atoms per unit volume in the two bodies. In this definition the value of $A_{H}$ is strongly related to the pairwise additivity of interacting atoms. It is assumed that the relation that gives the interaction energy between two single atoms is still valid for every individual atom in a body, and therefore pairwise additivity is allowed. Although this assumption is not completely correct, the geometrical results remain valid.

The geometry terms as given in equations (B.3) and (B.4) are derived for half space bodies. In reality bodies are finite. Especially in the case of galling and other material transfer mechanisms, the material that is transferred may consist of very small particles. So the question arises as to which amount interaction forces are geometry dependent and to what extent they are material dependent. The interaction energy is proportional to the inverse of the distance between the atoms to the $6^{\text {th }}$ power, so the interaction influence reduces very greatly as a function of distance. From the calculations presented in appendix B it follows that the equations (B.3) and (B.4) already give reasonable answers in case where the bodies are an order of magnitude larger than the gap between the bodies. Therefore, if wear particles come into contact with the tool, in such a way that the distance between the particle and the tool is approximately the atomic distance, the equations (B.3) and (B.4) already give good approximations since the wear particle has dimensions of about $10 \mathrm{~nm}$. As a result, it can be concluded that macroscopic adhesion relations and quantities can be used as long as the wear particle dimensions exceed $10 \mathrm{~nm}$.

\subsubsection{Surface and interfacial energy}

Material transfer is dependent on the bonds between the materials in contact. When chemical reactions are absent, the relevant bonds are physical bonds. The strength of these bonds is determined by the interfacial and surface energies. The work of adhesion, that is the work to be done in order to separate two surfaces, is:

$$
\Delta \gamma_{a b}=\gamma_{a}+\gamma_{b}-\gamma_{a b}
$$

The work per unit area $\Delta \gamma$ consists of the work to form a surface of material $a$ and of material $b$, the surface energies $\gamma_{a}$ and $\gamma_{b}$. The original interface disappears and so the interfacial energy $\gamma_{a b}$ is released. From equation (4.21) it follows that for a strong bonding between two materials, the value of $\gamma_{a b}$ should be small compared to the sum of $\gamma_{a}$ and $\gamma_{b}$. If equation (4.21) is used in the case where $a$ and $b$ are the same material, no initial interface exists, so the term $\gamma_{a b}$ is zero and $\Delta \gamma_{a b}=\Delta \gamma_{a a}=2 \gamma_{a}$.

A number of relations are found that approximate the surface energy to other physical quantities. In [38] it is shown that for pure metals in the cold worked state the value of $\gamma$ is related to the hardness as $\gamma \sim H^{1 / 3}$. In [31] and [32] the surface energy of $3 \mathrm{~d}$ metals (a group 
of metals in the periodic system containing iron and zinc) is given as a function of the electronic work function, the values of which can be found in a handbook as [18].

Adhesive forces between metals are dependent on the mutual solubility of a metal couple in the case where two dissimilar metals are contacting. If two metals have high mutual solubility, adhesion forces between these metals will be strong. This means that for such a metal couple the interfacial energy is low and the work of adhesion, needed to separate the surfaces, is high. If the mutual solubility of the metals is low, the interfacial energy is high and the work of adhesion is low. In figure 4.21, the compatibility of metal pairs is shown, according to [38].

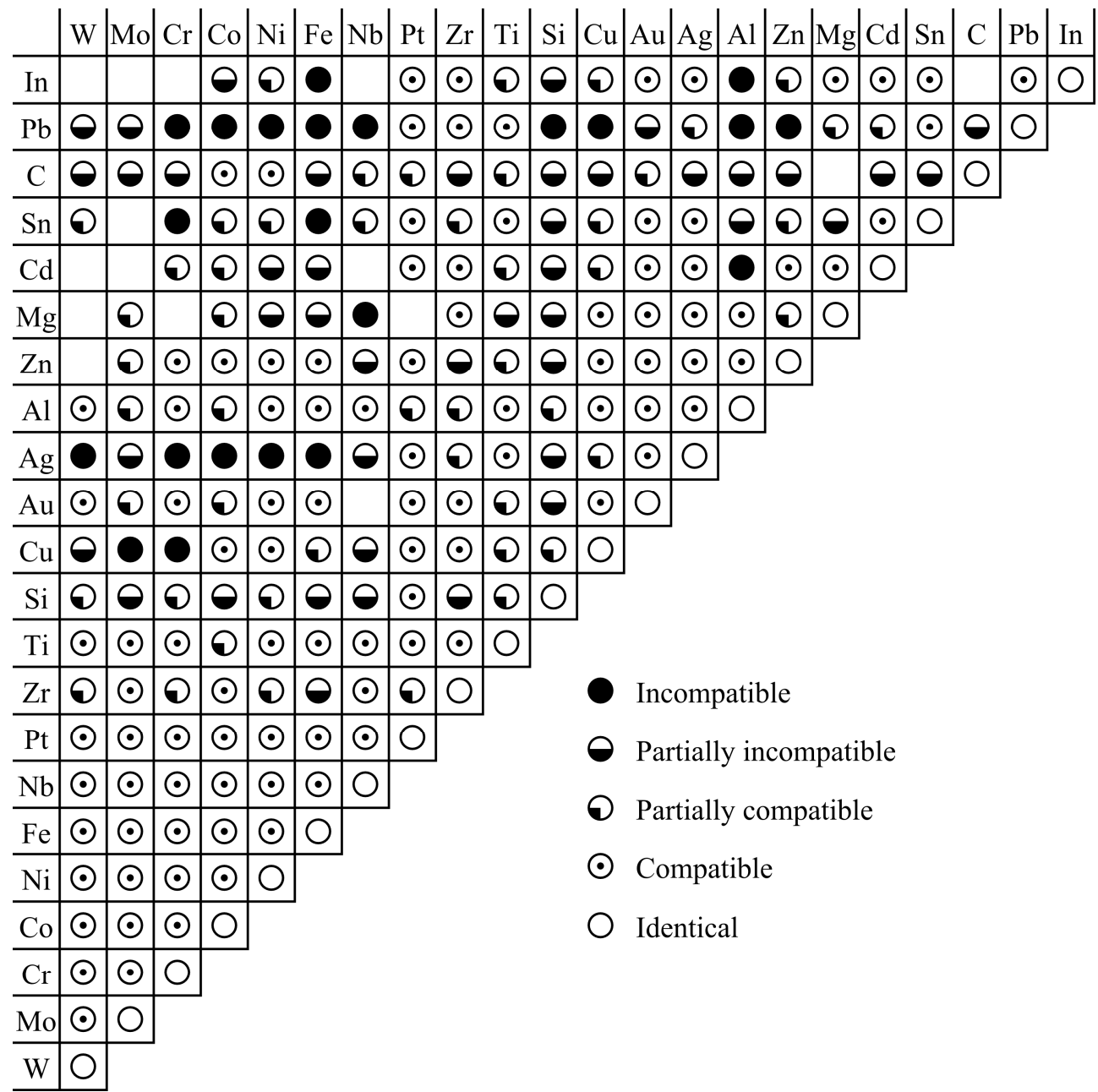

Figure 4.21. Compatibility pairs for elemental metals and a few non-metals, after [38]. 
The mutual solubility of metals is used to determine the work of adhesion, because it is in general very difficult to determine $\gamma_{a b}$. To determine the work of adhesion without using $\gamma_{a b}$, the following relation is used:

$$
\Delta \gamma_{a b}=c_{m}\left(\gamma_{a}+\gamma_{b}\right)
$$

In equation (4.22) $c_{m}$ is the compatibility parameter. Using this parameter, $\gamma_{a b}$ is made a function of $\gamma_{a}$ and $\gamma_{b}$. In [38] the parameters for the different categories, as shown in figure 4.21, are as given in table 4.2. In this table the values are given of $c_{m}$ and also the definition of the different solubility categories. In table 4.2 only a discrete set of values are given for $c_{m}$. In fact, these values only give an indication, because terms like compatible, partially compatible and so on are also ranges, with only chosen bounds without really physically strict bounds.

\begin{tabular}{|l|l|l|l|}
\cline { 2 - 3 } \multicolumn{1}{c|}{} & \multicolumn{2}{l|}{ solubility } & \multirow{2}{*}{} \\
\cline { 2 - 4 } \multicolumn{1}{c|}{} & liquid & solid (room temperature) & $c_{m}$ \\
\hline Identical metals & & & 1.00 \\
\hline Compatible metals & yes & $>1 \%$ & 0.50 \\
\hline Partially compatible metals & yes & 0.1 to $1 \%$ & 0.32 \\
\hline Partially incompatible metals & yes & $<0.1 \%$ & 0.20 \\
\hline Incompatible metals & no, two phases & & 0.12 \\
\hline
\end{tabular}

Table 4.2. Properties of mutual solubility categories of pure metals, after [38].

\subsubsection{Surface and interfacial energy in practice}

The values given above deal with the situation of pure metals in a clean environment. In engineering contacts, this will not in general be the case. At first, in a metal-metal contact, the bodies are mostly not created from a single element, but an alloy. Another aspect that has even more effect on the adhesion is the contamination on the surfaces. This contamination can be caused by an externally added substance, like a lubricant, but also by oxidation or other environmental influences.

An alloy has other surface properties than the pure metals from which it is composed. For the surface energy of an alloy it cannot be simply stated that it can be obtained by a weighted average of the surface energies of its elements. In [30] graphs are shown of the surface energy of some binary alloys as a function of the surface area fraction of one of the elements. These graphs do not show a linear relation. Using thermodynamic relations, the surface energies are estimated. Although a good estimation of the surface energy costs more effort, a weighted average of the surface energies of the elements gives a first rough approximation. 
Looking at the surface in more detail, the surface consists of a layered structure. One layer can be the base material with another grain structure, but also a layer of contamination from the environment. In the literature models can be found of this layered structure, such as the 4-zone model [6]. This model is shown in figure 4.22.

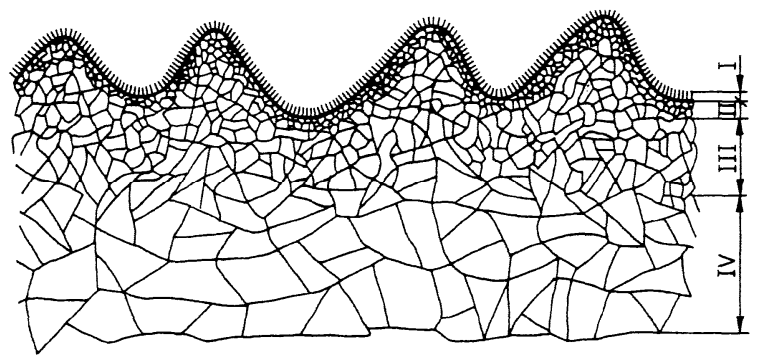

Figure 4.22. The 4-zone surface layers model, from [6].

These layers are:

- Layer I is formed by adhesion of particles from the environment like oxygen, nitrogen and water. The thickness of the layer does not exceed several ångströms.

- Layer II is formed by a mixture of bulk material with fine dusts, tool wear material and coolant-lubricant liquid. The thickness of the layer is about 1 to $10 \mathrm{~nm}$.

- Layer III contains only grains of the bulk material, which became deformed due to machining of the surface. The thickness of the layer is about $5 \mu \mathrm{m}$, but can be much thicker in the case of rough machining.

- Layer IV contains undeformed material, but is an influenced zone, for example by heat.

The layered structure above is one example of a model that describes the composition of the materials in the surface region. A number of other models are available with another number of layers. Some examples are also given in [6]. Most of the time, these models split up one layer into more layers to get more details, or the other way round, layers are combined to get a more generalized layer. The extra layers can be an oxide layer, which can be a part of layer II as given in figure 4.22, and an adsorbed layer on top of layer I, consisting of polarized particles of organic origin from lubricants.

In [33] the effect of layer I is clearly shown. Experiments are performed where two pure metals come into contact under ambient conditions. The samples have been cleaned carefully. If the friction is measured immediately after the samples come into contact, the friction is much lower than when the samples are first rubbed over each other. Examination of the surface shows no differences after both tests. In both cases the surface structure still originates from the polishing process. It is assumed that the formed surface layers are responsible for the different frictional behaviour. 
The same effect can be seen as surface energies are compared from the metal and its oxide. The surface energy of bare metals is in the order of $1 \mathrm{~J} \mathrm{~m}^{-2}$. Some values reported in [26] are $1.1 \mathrm{~J} \mathrm{~m}^{-2}$ for aluminium and $2.4 \mathrm{~J} \mathrm{~m}^{-2}$ for iron. The surface energy of aluminium oxide will be approximated using the value of $A_{H}$. According to [7] the value of $A_{H}$ is $15 \cdot 10^{-20} \mathrm{~J}$. The surface energy can be approximated, using [26]:

$$
\gamma \approx \frac{A_{H}}{24 \pi(0.165 \mathrm{~nm})^{2}}
$$

So, the value of $\gamma$ is approximately $73 \mathrm{~mJ} \mathrm{~m}^{-2}$. From these values, it follows that it takes much more energy to form a metal surface from splitting the material, than to form a metal oxide surface by splitting it. In the other way round, it is much more likely that two clean metal surfaces adhere, than to attach its oxides. Another problem in adhesion of oxides is the alignment of the lattices, which reduces the adhesion possibilities.

The above shows an approximation of the surface energy of aluminium oxide. This is only an approximation and not the real value. In general it is difficult to obtain a reliable value for the surface energy of metal oxides. These energies are very sensitive to the surface structure of the oxide. In the bulk of the material, an oxide is a regular structure, but at the surface this can be disordered. A different ordering of the surface can be responsible for different surface properties. Therefore, in different experiments, different values for surface energy can be obtained, for example, a surface obtained by cleavage of an oxide may result in other values than an 'original' oxide surface [23].

From the examples, it follows that contaminations and surface layers strongly lower adhesion. So in practice, the adhesion derived from clean metals as in the bulk give too high values in most cases. But in the case of galling, these high values are relevant, because the metals are in contact when the oxide or other contamination layers have been removed by mechanical contact and plastic deformation. Because continuous shear takes place in the wedge formation regime, the contact is modelled as the contact between two bare metals.

So in the following, the surface energy of bare metals will be taken as values for the surface energy. These values are given in table 4.3.

\begin{tabular}{l|l} 
Material & Surface energy $\gamma\left[\mathrm{J} \mathrm{m}^{-2}\right]$ \\
\hline Iron (used for tool steel) & 2.4 \\
Aluminium & 1.1 \\
Zinc & 0.99
\end{tabular}

Table 4.3. Surface energy of used metals, [26] and [46]. 


\subsection{Lump growth model}

\subsubsection{Introduction}

Material transfer is one of the reasons why the geometry of an asperity can change. Adhesion, discussed in section 4.4, is one of the basics of material transfer in the galling mechanism. In the case of material transfer, a lump can be formed on a tool asperity, which in turn, can scratch in the sheet that comes into contact with the lump. In this section a lump growth model will be developed.

The basis of the model is the lump growth model of De Rooij [39]. This model is described in section 2.3.2. This model is based on lump growth due to material transfer of wear particles generated in the wedge regime. The reason why material transfer takes place in wedge formation is explained in section 4.4.3. In [39] asperities are modelled with a spherical geometry. Further, lump growth causes only an increase in height of the asperity. In the model presented in section 4.5.3 and succeeding sections, the geometry of an asperity is formulated as a polyhedron with a hexagon base that is able to describe beside growth in height, also growth in width and length. The increase of these dimensions is a function of the volume of transferred material and a stability formulation of the asperity based on the analyses presented in section 4.3. The volume that is transferred due to adhesion is discussed in section 4.5.2. In section 4.5.3 the asperity geometry with the hexagon base is presented and the deposition of the transferred material on it in section 4.5.4. In section 4.5.5 the determination of the stresses in the hexagon base are determined, that are used in section 4.5.6 to determine the stability and the redistribution or (partly) shear off of the transferred material. In section 4.5.7 the influence of different parameters is shown. Lump growth presented in these sections deals only with the single asperity case. The multi asperity situation is described in chapter 5 .

\subsubsection{Volume of transferred material}

Lump growth is based on plastic deformation and the conditions of adhesion. In [39] it is assumed that the amount of material that is transferred is proportional to the adhesion force. The following steps are made:

1) It is assumed that material transfer only happens in the wedge formation regime. The wedge regime is defined by relations (2.9a) and (2.9b) and shown in figure 2.10. The material available for transfer will be calculated by the wear rate $k_{w}$. The wear rate is defined as:

$$
k_{w}=\frac{V_{\text {wear }}}{l_{\text {slide }} F_{n}}
$$


The wear rate in the wedge regime is according to [10]:

$$
k_{w}=\frac{1}{2 k} \frac{\sin ^{2} \theta+\frac{1}{2} \sin 2 \theta}{1+\sin 2 \theta}
$$

In equation (4.25) $\theta$ is defined as given in figure 2.7. Further, the shear strength $k$ is related to the hardness of the material, according equation to (4.11) by:

$$
k=0.18 H
$$

2) Only a certain fraction $m$ of $V_{\text {wear }}$ will be deposited on asperities on an area with size $A$, where $A$ is defined as $\pi \beta^{2}$ and $\beta$ the summit radius of the asperity. The height increase $\Delta s$ of the lump can then be expressed as:

$$
\Delta s=\frac{m V_{\text {wear }}}{A}=\frac{m l_{\text {slide }} \cdot k_{w} \cdot F_{n}}{A}
$$

The height increase is the only geometry change of the asperity. The size of $A$ results in a height increase $\Delta s$ of the whole growing asperity, assuming that the asperity is half a sphere with radius $\beta$ that is attached to the bulk material of the tool.

3) Further, it is assumed that $m$ is proportional to the adhesion force. In [39] $\mathrm{m} \cdot \mathrm{l}_{\text {slide }}$ is substituted by $m_{1}$. To make $m_{1}$ a function of the adhesion force, $m_{1}$ is substituted by $m_{2}$ times the adhesion force $F_{a}$ :

$$
m_{1}=m_{2} F_{a}
$$

Adaptations to the model of De Rooij have to be made, as indicated in the introduction. Two issues arise about the material transfer. The first issue deals with the volume that will be transferred. The second issue is how the transferred material is distributed over the asperity. Only the determination of the volume will be given here. The distribution will be discussed in section 4.5.4.

In equation (4.27) is shown that only a fraction $m$ of $V_{\text {wear }}$ will be deposited on the tool surface. In equation (4.28) it is assumed that $m$ is proportional to $F_{a}$. In [39] the value of $F_{a}$ is calculated using the relations given in [27] which are given in equation (2.21). These are relations that are valid for a sphere in contact with a flat plane. For the case of a polyhedron these relations cannot immediately be used. To get a value of $F_{a}$ or another value that is proportional to $F_{a}$, another strategy has to be followed. To get such a value, it is assumed that the contact of the asperity with the plastic material can be seen as a summation of a number of flat-on-flat contacts. According to [26] (and derived in appendix B) the work of adhesion per unit area between a flat-on-flat contact is: 


$$
W=\frac{-A_{H}}{12 \pi D^{2}}
$$

In relation (4.29) $D$ is the separation between the two surfaces. This relation forms the basis of equation (4.23), where the value of $D$ equals $D_{0}(=0.165 \mathrm{~nm}) . D_{0}$ is the separation between surfaces, when they are in contact at the atomic scale. From relation (4.29), $F_{a}$ can be derived by taking the derivative of $W$ :

$$
\frac{F_{a}}{A}=\frac{d W}{d D}=\frac{A_{H}}{6 \pi D^{3}}=\frac{-2}{D} W
$$

From equation (4.30) it follows that $F_{a}$ is proportional to $W$, for a fixed value of $D$. Relating this equation to $\gamma$ results in the following expression for the adhesive stress:

$$
\frac{F_{a}}{A}=4 \gamma \frac{D_{0}{ }^{2}}{D^{3}}
$$

From equation (4.31) follows that $F_{a}$ is proportional to $\gamma$. Further, it can be seen that the adhesion stress decreases with the third power as a function of the separation of the contacting surfaces. That means that a disturbance has a strong effect on $F_{a}$. In fact, using equation (4.31) gives too high values of adhesion force between surfaces, because it exceeds the ultimate tensile strength a lot. In practice disturbances always exist in the lattice, like dislocations. Another aspect is the metallic bonding. This results in the high value of $\gamma$ in the case of metals. The effect of the metallic bonding dominates for separations up to $0.5 \mathrm{~nm}$. For larger separations the Van der Waals interaction dominates. Equation (4.31) is derived on the basis of Van der Waals interaction. The value of $\gamma$ in that relation is too high, because the contribution of the Van der Waals interaction to $\gamma$ in the case of metals is low. In both cases, the metallic bonding and the Van der Waals interaction, $F_{a}$ is proportional to $\gamma$ and decreases very fast as a function of the separation. Therefore, in the current model it is assumed that the volume of the deposited material is proportional to the adhesion energy multiplied by the size of the contact area.

Following the steps that are formulated above on the basis of [39] and assuming that the deposited volume is proportional to the adhesion energy and contact area, the following relation can be constructed:

$$
V_{\text {transfer }}=m V_{\text {wear }}=c_{f r} \cdot \Delta \gamma \cdot l_{\text {slide }} \cdot k_{w} \cdot F_{n}
$$

In equation (4.32) the variables $l_{\text {slide }}, k_{w}$ and $F_{n}$ are responsible for the wear volume due to wedging as given in equation (4.24). To determine the wear rate the value of $\theta$ is needed. How to calculate $\theta$ will be given in section 4.5.4. The multiplication of the variables $c_{f r}$ and $\Delta \gamma$ gives the fraction of $V_{\text {wear }}$ that deposits on the asperity like $m$ in equation (4.27). Now, 
the fraction is not related to $F_{a}$, but to $\Delta \gamma$. This prevents that scaling of the asperity lead to scaling of $V_{\text {wear }}$ by $F_{n}$ and $m$ and so $V_{\text {transfer }}$ will scale quadratic.

The value of the constant $c_{f r}$ is more or less coupled to the value of $m_{2}$. If the fraction $m$ of equation (4.27) and (4.32) can be taken as equal, the following relation is obtained, from which the relation between $m_{2}$ and $c_{f r}$ becomes clear:

$$
\frac{m_{2} F_{a}}{l_{\text {slide }}}=c_{f r} \cdot \Delta \gamma
$$

\subsubsection{Geometry of the hexagon based lump}

In this section the geometry of the asperity and the deposition of the transferred material will be discussed. For the geometry of the asperity a polyhedron with a hexagon base will be used, an extension of the asperity geometry as developed in section 4.3. The volume that is deposited on this asperity will be determined using equation (4.32). The latest step discussed in this section is the deposition of the transferred material. This deposition concerns only the transfer of the adhered material. The final geometry of the transferred material will be determined by stress analyses as given in succeeding sections.

In section 4.3 an asperity model is formulated with a symmetrical pyramidal geometry. For this geometry mechanical stability analyses are performed. The ideas that are worked out in that section will be used for the development of the asperity geometry that will be presented here and for the stress and stability calculations in the follow sections. The geometry will be extended because of some geometrical limitations of a symmetrical pyramid. The symmetry axis of the pyramid should be in line with the sliding direction of it. This limits usability of the geometry to an isotropic roughness with contact spots with comparable length and width dimensions or with an anisotropic roughness that is aligned with sliding direction. Therefore a more flexible geometry will be formulated, that uses a lot of the properties of the symmetrical pyramid, but can be used in more general situations.

The asperity that will be used in the model has a hexagon base as is shown in figure 4.23. The asperity can be split up into three parts. The parts I and III are a pyramid and part II is a prism. The apexes of the pyramids are connected to the prism. The asperity can be oblique as given by the angle $\alpha$. The highest points of the asperity are formed by $G$ and $H$. The points $G$ and $H$ have the same $x$ and $y$-coordinates as $O$ and $O^{\prime}$, points on the line $A D$. The lengths $w_{\mathrm{I}}, w_{\mathrm{II}}$ and $w_{\mathrm{III}}$ are each independent, although in the galling model $w_{\mathrm{I}}$ and $w_{\mathrm{III}}$ will be taken as equal for simplicity. Further, the line $G H$ is horizontal and the lines $B C$ and $E F$ are parallel to the line $A D$. In this manner the geometry gives more flexibility to describe different asperities. The geometry of the pyramid asperity of section 4.3 can still be described, by setting the values of $w_{\mathrm{II}}$ and $\alpha$ to zero. Further, more elongated asperities can be described and with different orientations. 

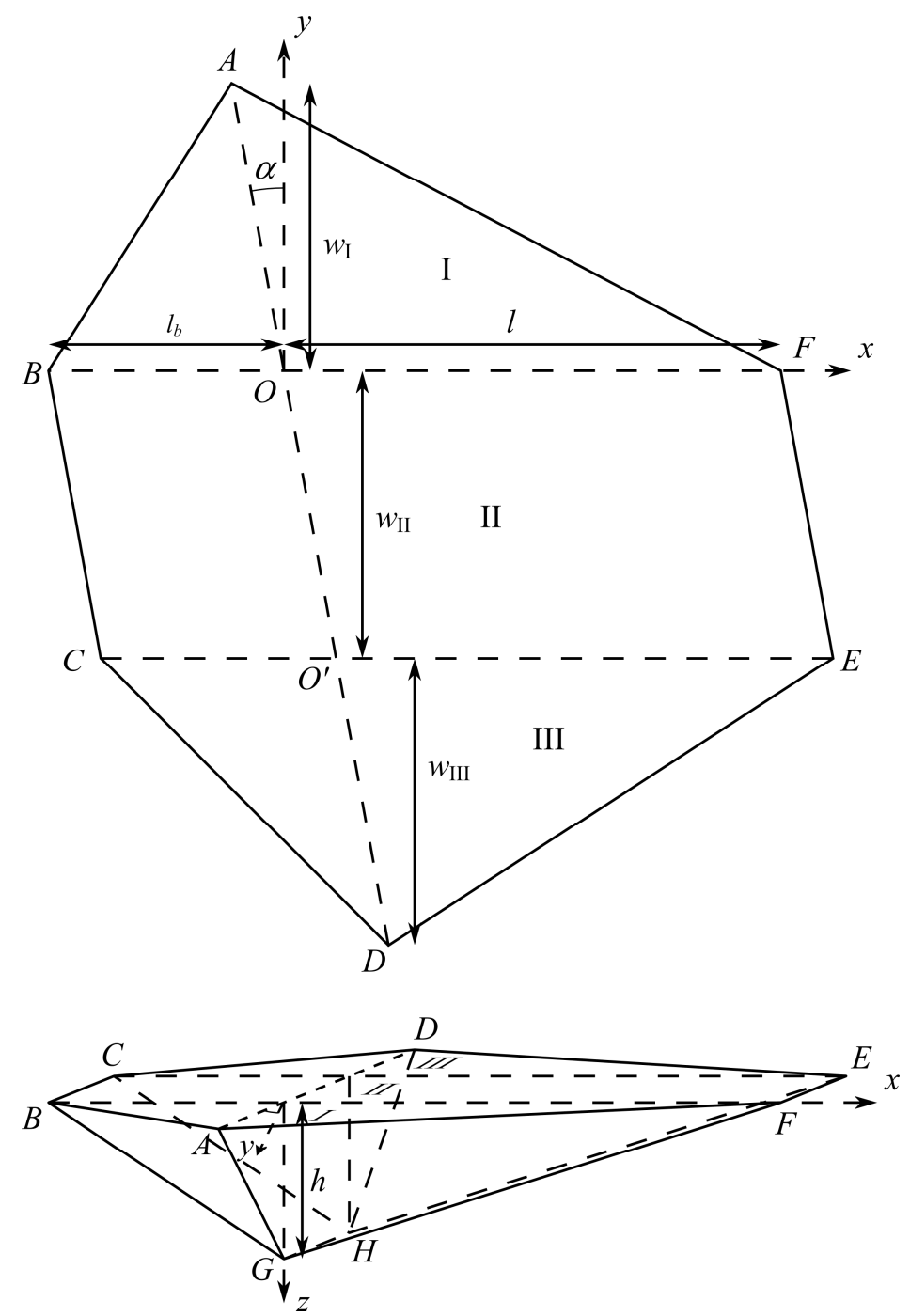

Figure 4.23. Dimensions of asperity with hexagon base.

\subsubsection{Deposition of transfer layer on hexagon based lump}

The next step is the deposition of transferred material. The volume of the material is given by equation (4.32). The wear rate as given in this equation can be found by equation (4.25). The attack angle $\theta$ can be found as the arctangent of $l$ divided by $h$. 
The question arises how this material is distributed over the surface. Equation (4.32) will be used to determine the volume, but also how the material is distributed. The terms that are responsible for the amount of $V_{\text {wear }}$ are $l_{\text {slide }}, k_{w}$ and $F_{n}$. The value of $l_{\text {slide }}$ is the same for the whole asperity. If a cross section of the asperity is taken parallel to the $x z$-plane the value of $\theta$ is the same on every $y$-coordinate within the asperity. In the case of an ideal plastic contact situation $F_{n}$ per unit area is constant (equals the hardness), the projected area on $x y$-plane is taken as the unit area. Therefore, the thickness of the transferred layer can be considered as constant in $z$-direction. That means that for steeper faces of the asperity the thickness of the layer is thinner measured perpendicular to the real surface.

The strategy that is given above to form a layer on the asperity with a constant thickness in $z$-direction will be followed, but has to be slightly adapted with respect to mass conservation, because edges are neglected in this strategy as is shown by the grey layer in figure 4.24. So, to fill the edges, the material has to be redistributed.
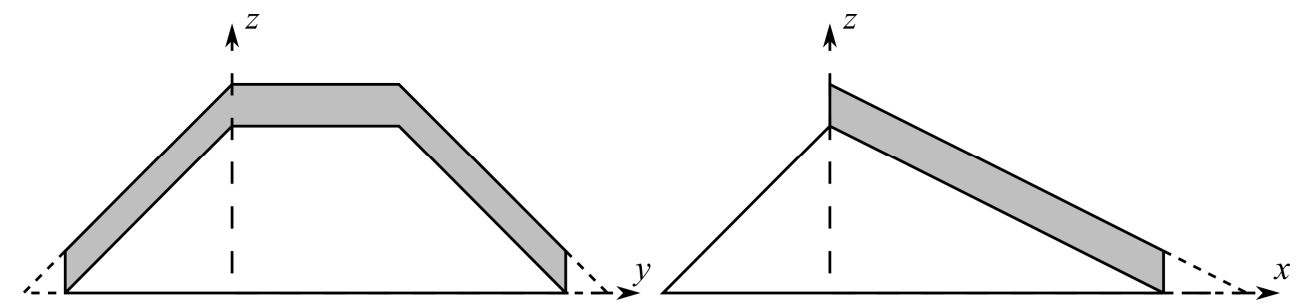

Figure 4.24. Schematic view of transferred layer on the contact faces as it has only a constant thickness in z-direction. The cross sections of the front view (left) and the side view are given.

The distribution of the transferred material will be done by a constant thickness in $z$-direction, but the thickness will be less than following from equation (4.32). The thickness will be reduced to fill the edges at the side and the front, as is given by the dashed edges in figure 4.24. The consequence for the geometry means that the width $w_{\text {II }}$ remains constant during lump growth, but that $w_{\mathrm{I}}$ and $w_{\mathrm{III}}$ increase. From the geometry follows that the volume of the frontal part of the lump (part where $x \geq 0$ ) is given by:

$$
V=\frac{h l}{6}\left(w_{\mathrm{I}}+3 w_{\mathrm{II}}+w_{\mathrm{III}}\right)
$$

The values of $h, l, w_{\mathrm{I}}$ and $w_{\mathrm{III}}$ all have to be scaled with the same factor to get the new volume due to the material deposition. The scaling factor $c_{s}$ can be found by solving the equation:

$$
\Delta V=\frac{h l}{6}\left(\left(w_{\mathrm{I}}+w_{\mathrm{III}}\right) c_{s}^{3}+3 w_{\mathrm{II}} c_{s}^{2}-\left(w_{\mathrm{I}}+3 w_{\mathrm{II}}+w_{\mathrm{III}}\right)\right)
$$


The lump that is grown now gets an upright back. Probably, this is not a mechanically stable situation. The next section discusses how the lump will deform due to contact forces after material deposition.

\subsubsection{Stresses inside hexagon based lump}

In section 4.5.4 it is described how the transferred material is deposited over the asperity. After this deposition step, the material only adheres permanently as it can sustain mechanical forces. This topic is already described in section 4.3 for a pyramidal asperity. In this section the results of section 4.3 will be used to determine the stresses inside the asperity, but are adapted such that these can be used for an asperity with a hexagon base.

From analyses that have been performed on a pyramidal asperity it was concluded that equation (4.8) can be used as fail criterion of the asperity when it is loaded due to plastic contact. In equation (4.8) the shear stress is determined parallel to the $x y$-plane. In section 4.3 this shear stress is determined on the basis of force equilibrium in the $x$-direction. In $y$-direction no net force is present due to the symmetrical load situation.

For the model of the asperity with the hexagon base a number of assumptions and results will be reused from the pyramid shaped asperity model. As far as possible, the assumptions as given in section 4.3.2 remain valid. That means in this situation that the coordinate directions still point in the same direction, although the origin is redefined. Further, the direction of the contact shear stress is determined by tangential vector on a contact face that minimizes the angle with the plastic flow in the far field. The plastic normal and tangential stresses remain unchanged. Assumptions on the basis of symmetry are not valid anymore. The result of the analyses with the pyramid shaped asperity, that the shear stress parallel to the $x y$-plane can be used as a fail criterion, will be reused. Now, both the stresses in $x$ and $y$-direction are of importance due to the absence of symmetry.

To calculate the forces the unit vectors as given in the equations (4.5) and (4.6) can be reused with some reinterpretation. The lengths $l, w$ and $h$ are real lengths in the case of the symmetrical pyramid. At the same time they are the locations where the contact plane crosses the coordinate axes. The $x$-axis is crossed at $x=l$, the $y$-axis at $y=w$ and the $z$-axis at $z=h$. From this point of view, for the unit vectors $l$ and $h$ can be reused, but $w$ has to be replaced by the following:

- Section I: $w \rightarrow \frac{w_{\mathrm{I}} l}{l+w_{\mathrm{I}} \cos \alpha}$

- Section II: $w \rightarrow l \cot \alpha$

- Section III: $w \rightarrow \frac{w_{\text {III }} l}{l-w_{\text {III }} \cos \alpha}$

Using the same strategy as in section 4.3 the following forces are derived: 


$$
\begin{gathered}
F_{x}=-p_{p l} h\left(\frac{1}{2} w_{\mathrm{I}}+w_{\mathrm{II}}+\frac{1}{2} w_{\mathrm{III}}\right) \\
-\tau_{p l}\left(\frac{1}{2} \sqrt{\left(w_{\mathrm{I}} l\right)^{2}+h^{2}\left(l+w_{\mathrm{I}} \tan \alpha\right)^{2}}\right. \\
+w_{\mathrm{II}} \sqrt{l^{2}+h^{2} \tan ^{2} \alpha} \\
\left.+\frac{1}{2} \sqrt{\left(w_{\mathrm{III}} l\right)^{2}+h^{2}\left(l-w_{\mathrm{III}} \tan \alpha\right)^{2}}\right) \\
F_{y}=-p_{p l} h \tan \alpha\left(\frac{1}{2} w_{\mathrm{I}}+w_{\mathrm{II}}+\frac{1}{2} w_{\mathrm{III}}\right) \\
+\tau_{p l} h^{2}\left(\begin{array}{c}
\frac{1}{2} \frac{w_{\mathrm{I}}\left(l+w_{\mathrm{I}} \tan \alpha\right)}{\sqrt{\left(l w_{\mathrm{I}}\right)^{2}+h^{2}\left(l+w_{\mathrm{I}} \tan \alpha\right)^{2}}} \\
+\frac{w_{\mathrm{II}} \tan ^{2}}{\sqrt{l^{2}+h^{2} \tan ^{2} \alpha}} \\
-\frac{1}{2} \frac{w_{\mathrm{III}}\left(l-w_{\mathrm{III}} \tan \alpha\right)}{\sqrt{\left(l w_{\mathrm{III}}\right)^{2}+h^{2}\left(l-w_{\mathrm{III}} \tan \alpha\right)^{2}}}
\end{array}\right)
\end{gathered}
$$

The derivation of these forces is given in more detail in appendix A. The tangential stresses parallel to the $x y$-plane in both directions are found by dividing the forces as given in equations (4.36a) and (4.36b) by the area of the plane $A B C D E F$, see figure 4.23:

$$
A_{A B C D E F}=\left(l+l_{b}\right)\left(\frac{1}{2} w_{\mathrm{I}}+w_{\mathrm{II}}+\frac{1}{2} w_{\mathrm{III}}\right)
$$

Dividing the forces by the area as given in equation (4.37) can result in an underestimation of the tangential stresses, because the plastic forces are not supported by the whole area $A B C D E F$. This is analogous to the situation described in section 4.3.4. To get the proper value for the stresses $l_{b}$ has to be replaced by $c h$.

The stresses that are determined may not exceed the material strength. Because of the absence of symmetry in the asperity, not only the tangential stress in $x$-direction, but also the stress in $y$-direction has to be taken into account for determining lump failure. The norm of the sum of both stress vectors will be used as the fail criterion.

To present the stresses in the plane $A B C D E F$, they are calculated for different orientations of the asperity related to the ploughing direction. From the elliptic base a hexagon is constructed. The construction of the hexagon on the basis of an ellipse will be described in chapter 5, where this strategy is used for the conversion from measured data of a surface to a set of hexagon based asperities. The idea of the fit is shown in figure 4.25. In this way it becomes possible to compare an equivalent asperity for different orientations. This cannot be done with only one hexagon, because the hexagon needs lines that are parallel to the 
ploughing direction, namely the lines $B F$ and $C E$ as given in figure 4.23 , so this hexagon base cannot be turned just like that.

The stresses in the plane $A B C D E F$ and its directions are presented in the figures $4.26,4.27$ and 4.28. Four situations with different asperity geometries are presented: two different shapes of the contact area and two heights. The contact areas are ellipses that are converted to hexagons. One shape of contact has a ratio of the major radius $a$ and minor radius $b$ of 1.5 , the other contact has a ratio $a / b$ of 10 . The normal load is kept constant.
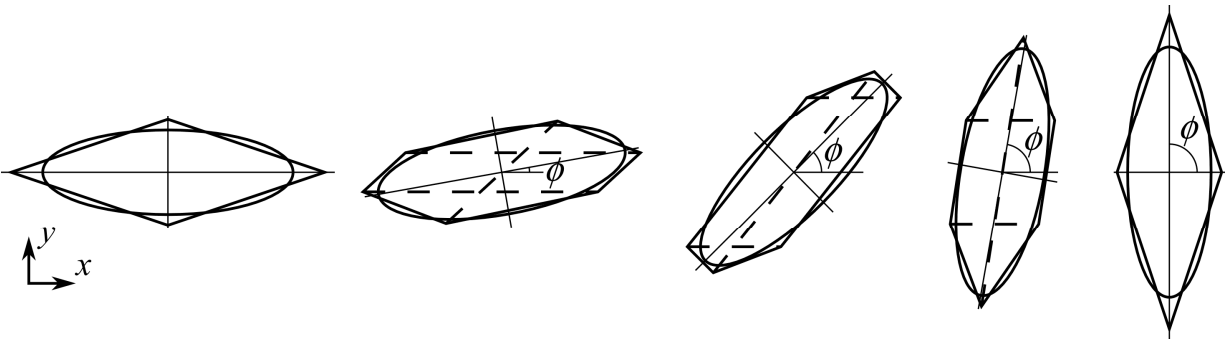

Figure 4.25. An ellipse with a hexagon fit for different orientations $\phi$. For $\phi=0^{\circ}$ and $\phi=90^{\circ}$ the dimension $w_{I I}$ is reduced to 0 . 

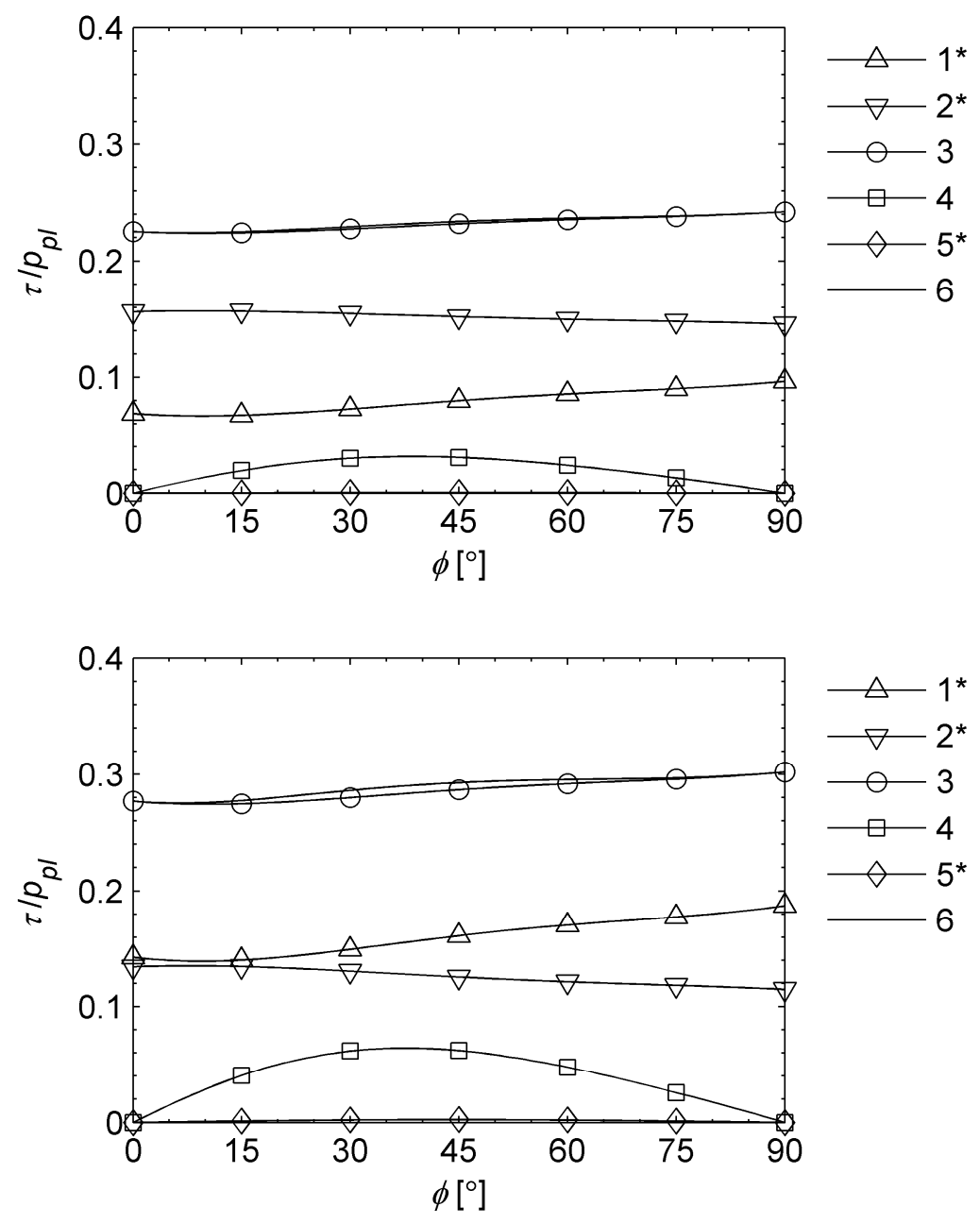

$b$

Figure 4.26. Normalized mean shear stresses as a function of the orientation $\phi$ on face $A B C D E F$ for $a / b=1.5$ and $\mu=0.18$ and two different heights: $h=0.1 b$ (a) and $h=0.25 b$ (b). The presented shear stress components in $x$-direction are caused by the plastic normal force (1), the plastic friction force (2) and the total plastic force in x-direction (3). The presented shear stress components in y-direction are caused by the plastic normal force (4) and the plastic friction force (5). The shear stress caused by the total force in y-direction is omitted, because it lies almost on line 4. The absolute value of the shear stress on face $A B C D E F$ is given by line 6. The asterisks (*) behind the numbers in the legend indicate that the values are negative, but are presented as positive. 

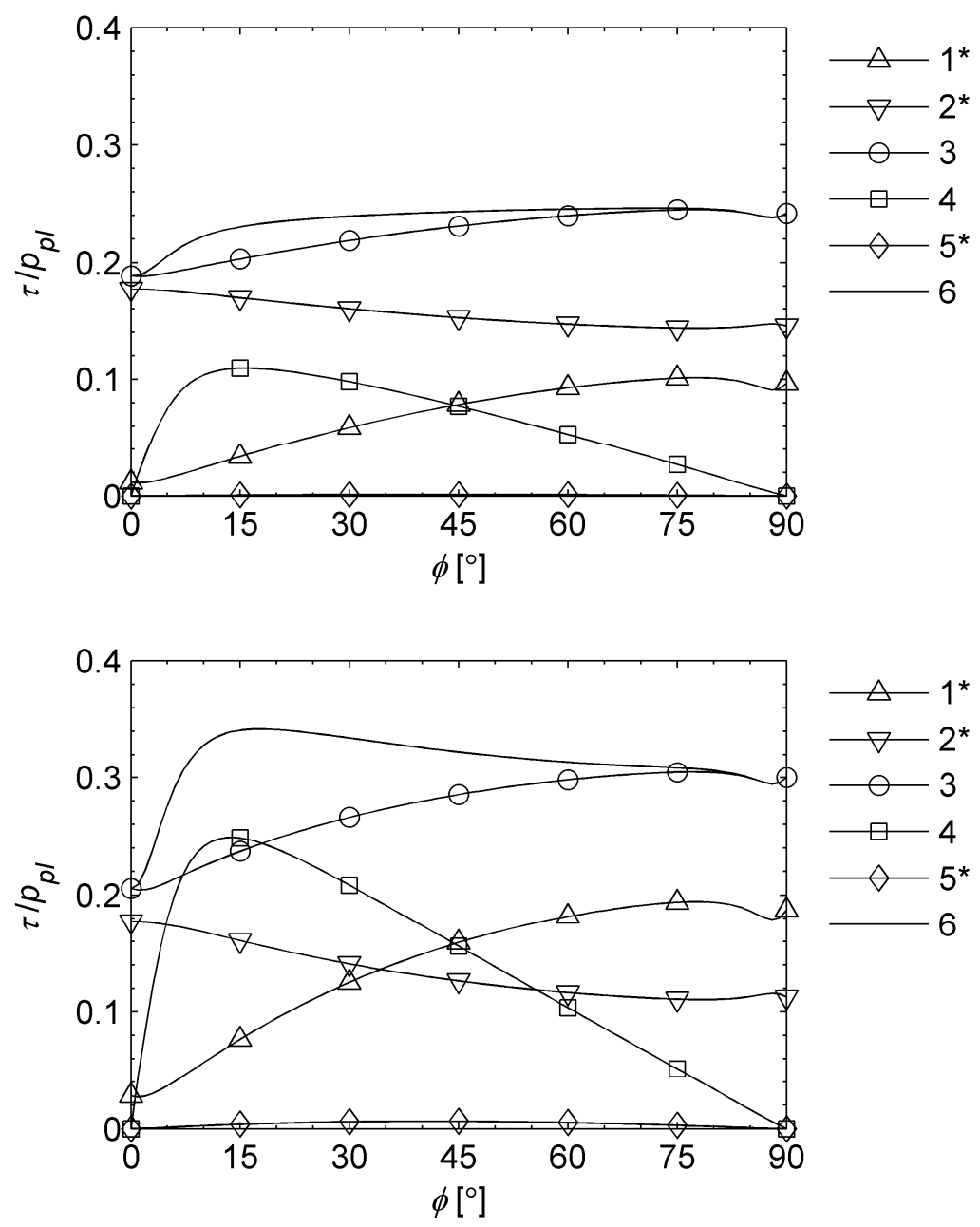

b

Figure 4.27. Normalized mean shear stresses as a function of the orientation $\phi$ on face $A B C D E F$ for $a / b=10$ and $\mu=0.18$ and two different heights: $h=0.1 b$ (a) and $h=0.25 b$ (b). The presented shear stress components in $x$-direction are caused by the plastic normal force (1), the plastic friction force (2) and the total plastic force in x-direction (3). The presented shear stress components in y-direction are caused by the plastic normal force (4) and the plastic friction force (5). The shear stress caused by the total force in y-direction is omitted, because it lies almost on line 4. The absolute value of the shear stress on face $A B C D E F$ is given by line 6. The asterisks (*) behind the numbers in the legend indicate that the values are negative, but are presented as positive. The meaning of the different lines corresponds to the lines of figure 4.26 . 

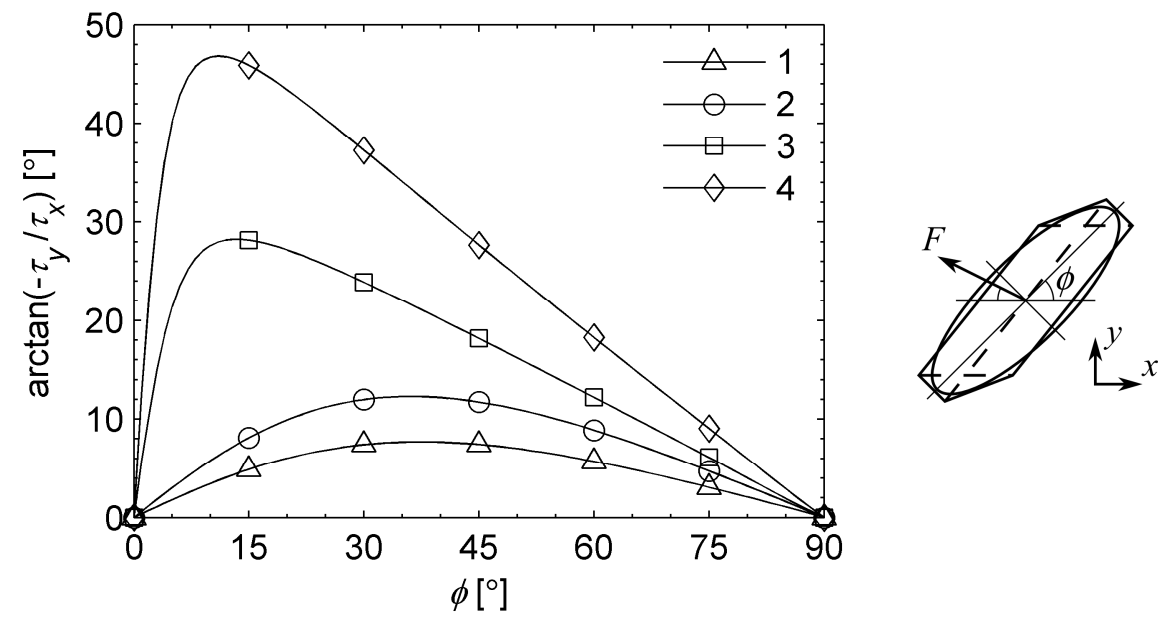

Figure 4.28. Orientation of the force on an asperity as a function of the orientation of the asperity itself. The angles of both axes of the graph are given in the figure at the right hand side. Four different asperity geometries are presented varying the ellipticity ratio $a / b$ and the height as a function of $b$. In all cases $\mu=0.18$. Presented are the geometries: $h=0.1 b$, $a / b=1.5(1) ; h=0.25 b, a / b=1.5(2) ; h=0.1 b, a / b=10$ (3) and $h=0.25 b, a / b=10$ (4).

The figures 4.26 and 4.27 present the effect of different geometries and orientations on the stress situation in the asperity. Here, only the orientations with a positive value of $\phi$ are shown, because the negative values give no extra information, only the stresses in $y$-direction get a sign change. In the graphs, the stresses are always presented with a positive value. In the case of negative values an asterisk (*) is given in the legend of the graphs. The graphs will be given some explanation in the same order as the graphs are given in the legends. The stresses in $x$-direction are given in the graphs numbered from 1 to 3. Graph 1 gives the stress that is caused by the plastic normal pressure on the contact planes, graph 2 gives the stress caused by the tangential stresses on the contact planes and graph 3 is the sum of both. For the stresses in $y$-direction only the stresses caused by the normal contact pressure and shear stress are given, in graphs 4 and 5, and the sum is omitted (nearly equal to 4). The last graph gives the norm of the vector summation of the stress in $x$ and $y$-direction.

From the graphs in figures 4.26, 4.27 and 4.28 some effects of geometry can be observed. In figure 4.26 stresses are plotted for the situation with a low value of $a / b$. Such geometry has the consequence that the asperity is almost loaded symmetrically, which results in a low shear stress in $y$-direction and the magnitude of the stress in $x$-direction equals almost the 
absolute value of the stress in the $A B C D E F$-plane. In the case of a high value of $a / b$, as is plotted in figure 4.27 , the orientation of the asperity has a clear effect on the shear stresses, because the asperity is not loaded symmetrically. The total load is for most orientations more or less the same, only if $\phi$ is near $0^{\circ}$ where the shear stress is lower, that is the case that the frontal area is minimal. The components of the stress in the $x$ and $y$-direction are clearly not constant. For $\phi=0^{\circ}$ the asperity is loaded symmetrically, so no net force acts in $y$-direction, but if the value of $\phi$ increases a bit, the net force acts in the positive $y$-direction. At one side the projected contact area increases, these are the contact areas of section I and II as given in 4.23 , but at the other side, the area of section III, it decreases. These projected contact areas (projected on the $x z$-plane) are of importance, because the plastic normal pressure is the dominant stress component in the $y$-direction. From figure 4.28 comparable conclusions can be drawn about the effect of a high value of $a / b$ on the direction of the stresses in the $A B C D E F$-plane. The height of the asperity is of most importance for the effect of the plastic normal pressure.

\subsubsection{Stability and material redistribution of the asperity}

In the former section, stresses are calculated. As long as the material is strong enough, a given geometry can be used to calculate the stresses. When the limits of the materials are reached, the stress is known and the maximum dimensions of the geometry have to be determined. Section 4.5.4 describes how a layer will be deposited on the asperity. The lump that forms on the asperity can only grow when it is strong enough and can withstand the plastic forces that act on it. To get a stable lump, the following is assumed:

- If the lump is not stable, material is removed to make it stable again, but none of the dimensions will be smaller than before the material deposition.

- The material strength is taken to be equal to the strength of the deposited layer.

- The rear side crumbles off, so the rear side gets a slope equal to $|\Delta z / \Delta x| \approx 0.5$. These assumptions will be explained in the following. The first assumption has the consequence that dimensions become smaller after initial material deposition. So, material redistribution is not taken into account where one dimension decreases in favour of another one. That dimensions cannot decrease to values lower than before the material deposition only has consequences in the beginning of lump growth. The asperity on which the lump grows can be too steep according to stability criteria and therefore shear off. But, generally speaking, the tool is harder than the sheet material. Therefore, it is assumed that the original asperity will be strong enough. Taking the strength of the deposited layer as a criterion, as is stated in the second assumption, the height of the lump will only exceed the original height of the asperity if the lump itself is strong enough. Once the lump on its own is stable, every cycle that a new layer is deposited, the assumption becomes superfluous that none of the dimensions will be smaller compared with the situation before the material deposition. 
The last assumption says that the rear side crumbles off. The value of $|\Delta z / \Delta x|$ is the inverse of $c$ as derived in section 4.3.4. In section 4.3.4 it is shown that the rear part of the asperity carries a limited amount of load, only till a length of $c h$ backwards of the loaded zone. The deposited layer can be supported in the same manner. The edge at the top can only be supported in this manner as the slope at the rear side is not steeper than $1 / c$. So the perpendicular edge as given on the right-hand side of figure 4.24 will not exist in the final situation.

From the assumptions given above, two extremes can be distinguished for the new geometry after material deposition and stability step. The most stable one is the one that $h$, $w_{\mathrm{I}}$ and $w_{\mathrm{III}}$ get the value as before the deposition of the transferred material and $l$ gets the new value that is obtained by the material transfer. The less stable geometry is formed as only the rear side of the transfer layer crumbles off. The latter has the consequence that due to crumbling off of the rear edge $l_{b}$ will increase and $h, w_{\mathrm{I}}$ and $w_{\mathrm{III}}$ decrease a bit in relation to the deposited layer. To calculate the new geometry after the stability step, a number of steps have to be made. The subscript 0 is used for dimensions of the situation before the layer deposition and the 1 as the situation between the deposition and stability step. The steps are as follows, using table 4.4:

1) Calculate the stresses using the dimensions for the most stable case as given in column (A). If the stresses are above the strength of the deposited layer, the final dimensions become the dimensions of column (A). Otherwise, go to step 2.

2) Calculate the stresses using the dimensions for the least stable case as given in column (B). If the stresses are below the strength of the deposited layer, the final dimensions become the dimensions of column (B). Otherwise, go to step 3.

3) Calculate the dimensions using the relations as given in column (C). The dimensions and the stresses can be calculated as a function only of $h$. The final dimensions are determined by the situation that the stresses are equal to the strength of the deposited layer. This cannot be determined explicitly, so this should be done using an iterative procedure.

In these calculations $l_{b}$ will be substituted by $c h$ instead of the real physical length in backwards direction. The stresses are determined by the vector sum of the shear stress in $x$ and $y$-direction. 


\begin{tabular}{|l|l|l|l|}
\cline { 2 - 4 } \multicolumn{1}{c|}{} & Most stable (A) & Least stable (B) & Otherwise (C) \\
\hline$l_{b}$ & $l_{b 0}$ & $l_{b}=\frac{l_{b 0}+l_{1}}{l_{b 0}+l_{0}} l_{b 0}$ & $c h$ \\
\hline$l$ & $l_{1}$ & $l=\frac{l_{b o}+l_{1}}{l_{b o}+l_{0}} l_{0}$ & $l=l_{1}+l_{b 0}-c h$ \\
\hline$h$ & $h_{0}$ & $h=\frac{l_{b 0}+l_{1}}{l_{b 0}+l_{0}} h_{0}$ & $h$ \\
\hline$w_{\mathrm{I}}$ & $w_{\mathrm{I} 0}$ & $w_{\mathrm{I}}=\frac{l_{b 0}+l_{1}}{l_{b 0}+l_{0}} w_{\mathrm{I} 0}$ & $w_{\mathrm{I}}=\frac{c h}{l_{b 0}} w_{\mathrm{I} 0}$ \\
\hline$w_{\mathrm{III}}$ & $w_{\mathrm{III} 0}$ & $w_{\mathrm{III}}=\frac{l_{b 0}+l_{1}}{l_{b 0}+l_{0}} w_{\mathrm{III} 0}$ & $w_{\mathrm{III}}=\frac{c h}{l_{b 0}} w_{\mathrm{III} 0}$ \\
\hline
\end{tabular}

Table 4.4. Dimensions of asperity after stability step. The values of $\alpha$ and $w_{I I}$ are omitted, because they do not change.

In the figures 4.29 to 4.31 some results of the lump growth model are shown for a single asperity in contact. The three cases that are shown in the figures are all the same, except the orientation of the asperity. Starting point for the asperity is again a paraboloid. The elliptic base is formed by the major and minor axis $a$ and $b$. The orientation $\phi$ is as given in figure 4.25. Further, it is assumed that the nominal separation of the contact surfaces is not changed by the lump growth of this lump, so the 'original' asperity penetrates the same amount in the plastic counter body and the scratch depth in the plastic body increases due to the extended lump. The physical values used in the model are given in table 4.5.

\begin{tabular}{|l|l|l|}
\cline { 2 - 3 } \multicolumn{1}{c|}{} & Parameter & Value \\
\hline Asperity parameters & $a$ & $2.5 \mu \mathrm{m}$ \\
\cline { 2 - 3 } & $b$ & $0.5 \mu \mathrm{m}$ \\
\cline { 2 - 3 } & $h$ & $0.3 \mu \mathrm{m}$ \\
\hline Material parameters & $T$ & 0.27 \\
\cline { 2 - 3 } & $H$ & $1 \mathrm{GPa}$ \\
\cline { 2 - 3 } & $\Delta \gamma$ & $1.1 \mathrm{~J} \mathrm{~m}^{-2}$ \\
\hline Other parameters & $l_{\text {slide }}$ & $0.1 \mathrm{~m}^{-5} \mathrm{~m}^{2} \mathrm{~J}^{-1}$ \\
\cline { 2 - 3 } & $c_{f r}$ & $1 \cdot 10^{-5}$ \\
\cline { 2 - 3 } & $\mu$ & 0.18 \\
\cline { 2 - 3 } & number of cycles $n$ & 5 \\
\hline
\end{tabular}

Table 4.5. Physical parameters used in galling model. 
Using the results of the calculation some characteristics of the model can be shown and the effect of different situations on the galling behaviour according to the model.

In the figures 4.29 to 4.31 , in the upper half, the vertical cross section is given at the $x z$-plane, in the lower half the horizontal cross section at the $x y$-plane. On the horizontal cross section an ellipse is shown. This ellipse is the original paraboloid asperity; the hexagon that is positioned on the ellipse is its hexagon fit that is used in the galling model. The triangle at $x=0$ on the vertical cross section belongs to the same fitted asperity. All lines in front of the hexagon and the triangle are deposited layers on the asperity. Every line gives the situation after one cycle. The parameters that are chosen are the ones the lump has already developed in a few cycles. In practice, the lump growth is much slower, otherwise the effects of galling would already become disastrous after some tens of products.

In all cases that are given in the figures the lump does not grow in height during the first cycle. The asperity is too steep and therefore not strong enough to enable it to resist the internal horizontal shear stresses. In the case of $\phi=10^{\circ}$ the height still does not increase during the second cycle. In these situations the lump grows according to the most stable geometry as is given in table 4.4. Once the lump is strong enough, the lump grows according to column $\mathrm{C}$ or even according to column $\mathrm{B}$ as is given in table 4.4. From the figures 4.30 and 4.31 it follows clearly that during the second cycle the lump grows according to column $\mathrm{C}$. This can be seen by the fact that the lump grows relatively more in horizontal direction than in the vertical direction. The last cycles still grow according to column $\mathrm{C}$, but this is not immediately clear from the figures, because the numerical values differ only one percent or less from the situation given in column B. In these cases the lump scales almost proportionally in all directions, except for $w_{\text {II }}$ which remains constant.

A difference in lump growth can be distinguished for the different asperity orientations. During the five cycles of material deposition, the total height increase is larger for a larger angle $\phi$. Two reasons are responsible for this effect. For small angles of $\phi$, the stresses in the asperity are higher due to a large net force in the $y$-direction. This has to be compensated by the formation of a low slope of the asperity. The second reason is a result of the first one. Due to the low slope, that means a small attack angle $\theta$, the wear rate decreases. As a consequence of the lower wear rate, less material is available for deposition on the asperity. In the application of galling, this means that the orientation of asperities will have consequences for the severity of galling. A transverse sliding asperity is more severe with respect to galling than a longitudinal sliding asperity. 


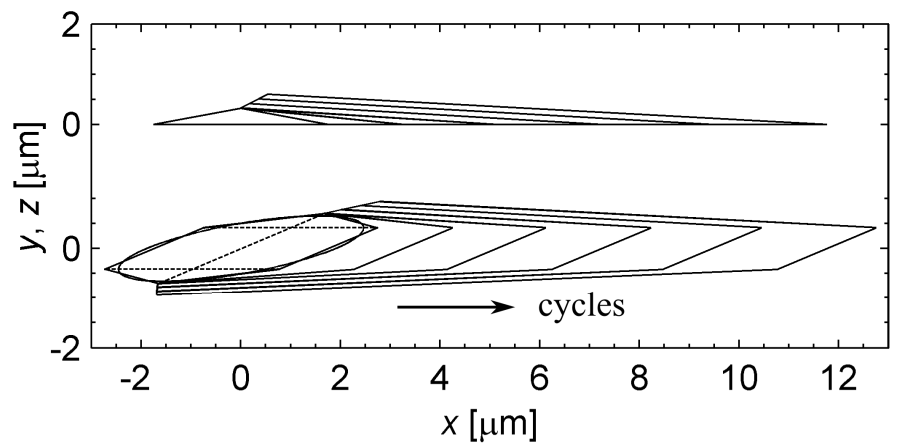

Figure 4.29. Lump development on asperity with orientation $\phi=10^{\circ}$.

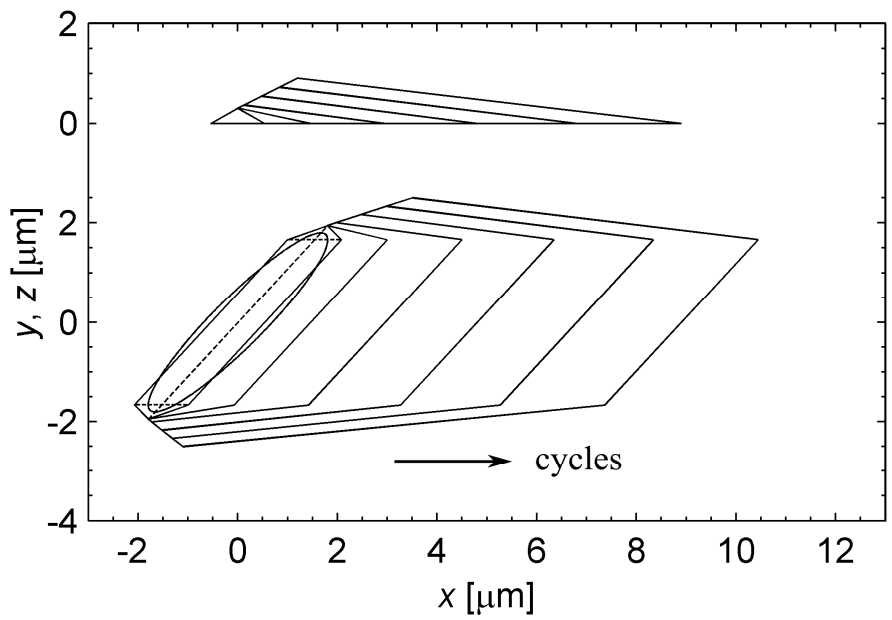

Figure 4.30. Lump development on asperity with orientation $\phi=45^{\circ}$. 


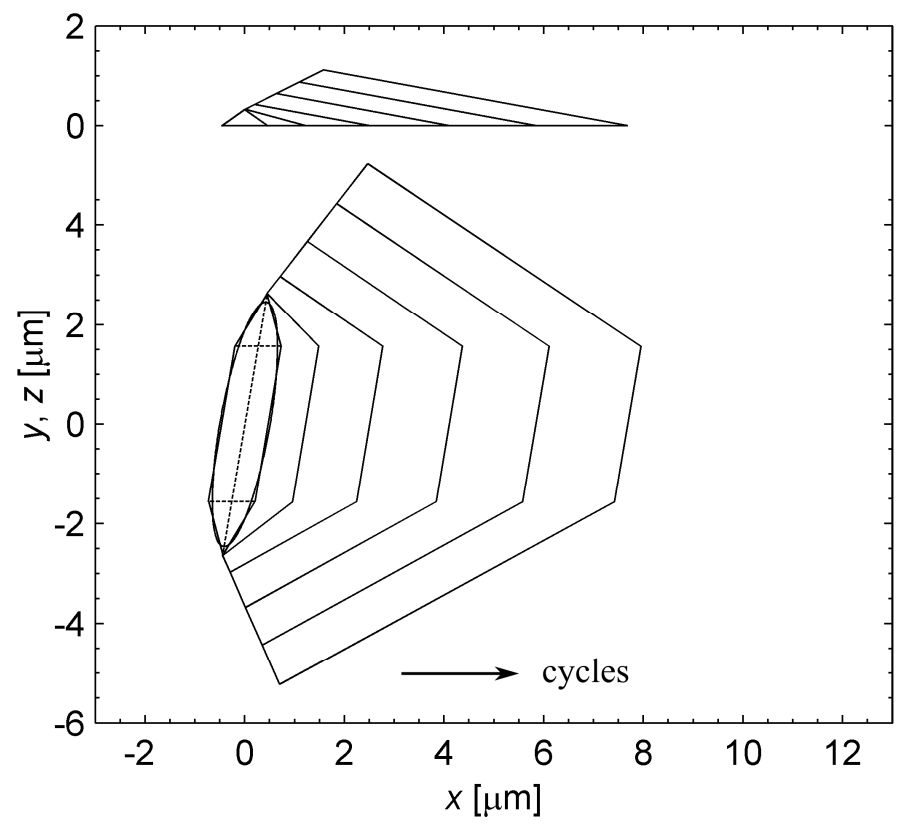

Figure 4.31. Lump development on asperity with orientation $\phi=80^{\circ}$.

\subsubsection{Influence of parameters}

The effect of different parameters in the model will be presented. Similar to the calculations belonging to the figures 4.29 to 4.31 , the physical quantities are as given in table 4.5 , unless another value is given.

The growing behaviour will be presented as a function of the transferred volume and the number of products. According to equation (4.32) the volume of the transferred material is proportional to the multiplication of $c_{f r}, \Delta \gamma$ and $l_{\text {slide }}$. As only these three quantities are changed in such a manner that the multiplication remains constant, the output of the model does not change in terms of transferred volume in this example. The number of products $n$, before unacceptable scratching occurs, is more or less related to $c_{f r} \cdot \Delta \gamma \cdot l_{\text {slide }}$. The multiplied factor gives the fraction of the transferred volume, but this volume is also proportional to $n$ (ignoring the change of geometry after each product that is formed). Therefore, as $c_{f r} \cdot \Delta \gamma \cdot l_{\text {slide }} \cdot n$ is constant, the output of the model should be almost constant. This effect is shown in figures 4.32 to 4.34 . The lines in the figures are difficult to distinguish, because of the very small differences. For all figures the lowest graph belongs to $n=100$ and the highest graph to $n=10000$. 


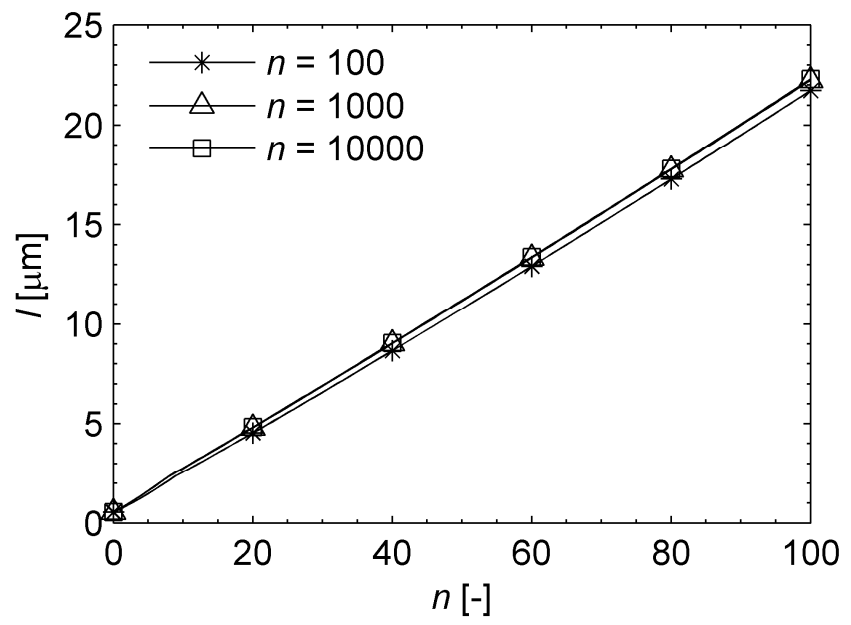

Figure 4.32. Development of the lump length l during lump growth. On the horizontal axis the number of formed products are given for the case that $n=100$. For the case $n=1000$ the numbers at this axis have to be multiplied by 10, for the case $n=10000$ the numbers have to be multiplied by 100. The values of $c_{f r} \cdot \Delta \gamma \cdot l_{\text {slide }}$ are respectively $1.1 \cdot 10^{-7}, 1.1 \cdot 10^{-8}$ and $1.1 \cdot 10^{-9} \mathrm{~m}^{-1}$. The orientation is given by $\phi=45^{\circ}$.

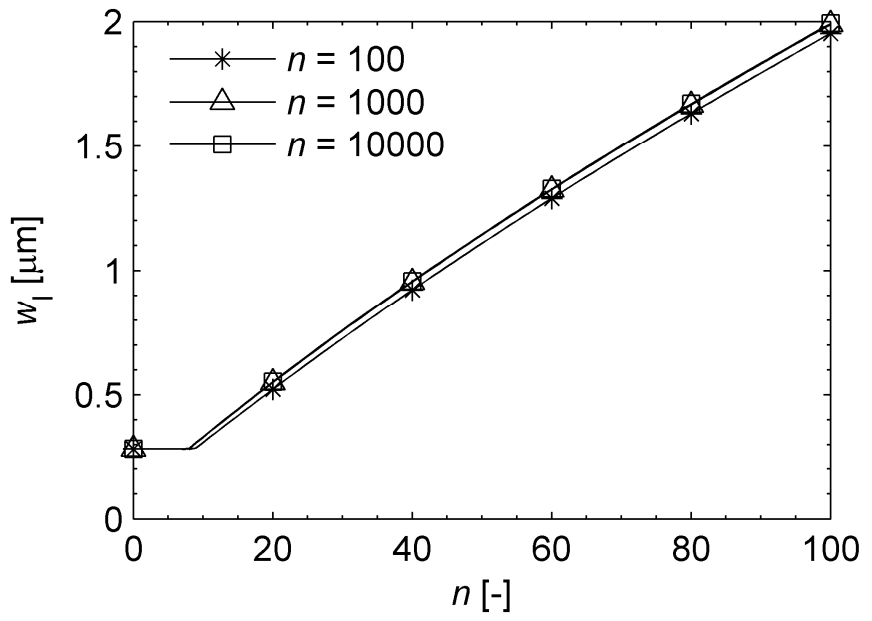

Figure 4.33. Development of dimension $w_{I}$ of the lump during lump growth. The other details are as given for figure 4.32. 


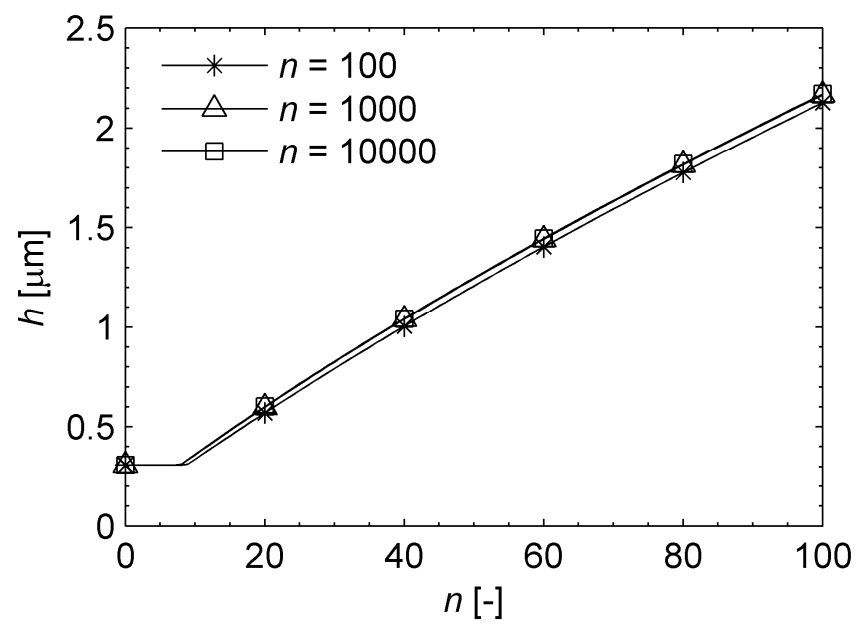

Figure 4.34. Development of the lump height h during lump growth.

The other details are as given for figure 4.32.

From the figures 4.32 to 4.34 it follows that first the lump grows in frontal direction, so the base of the lump becomes more stable. After the base is stable enough, the lump grows in normal direction, such as also shown in figures 4.29 to 4.31 .

Because of the almost constant character for the situations given in figures 4.32 to 4.34 the following figures are given only for $c_{f r} \cdot \Delta \gamma \cdot l_{\text {slide }}=1.1 \cdot 10^{-6} \mathrm{~m}^{-1}$ and $n=1000$.

In figures 4.35 and 4.36 the effect of asperity orientation on lump growth is shown. Roughly speaking, if $\phi<45^{\circ}$ the lump grows relatively slowly, if $\phi>45^{\circ}$ the lump grows faster in height. For small values of $\phi$ the lump is strongly asymmetrically loaded in $y$-direction as also is shown in figure 4.27 and so, the lump shears off in sideward direction. This effect prevents a fast lump growth. Only if $\phi$ is around $0^{\circ}$ the load in $y$-direction is (almost) symmetric, so the lump can grow faster again. In the case where a tool has grinding grooves in the direction of sliding between the tool and the workpiece, the lump growth already will be reduced with a small misalignment of the grinding grooves and sliding direction. 


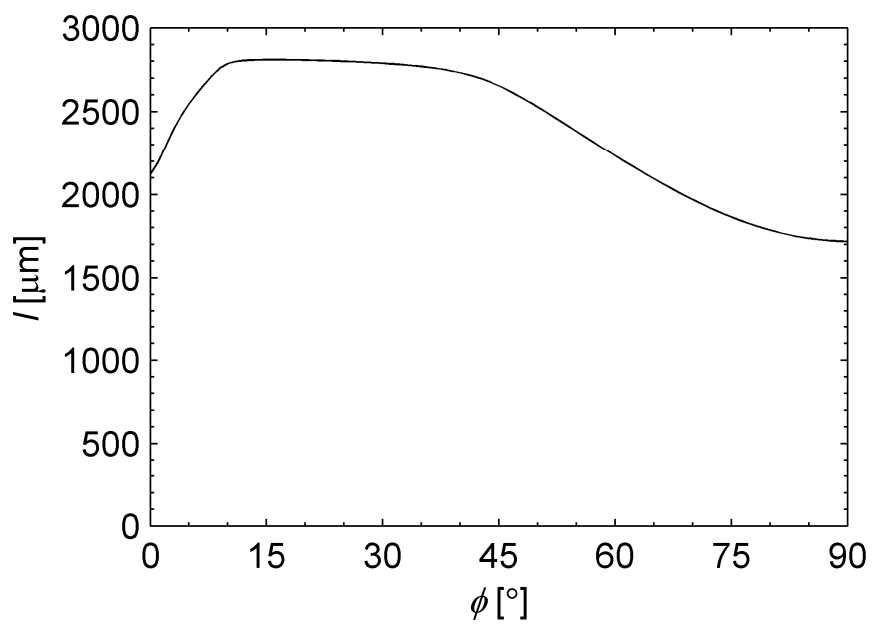

Figure 4.35. Lump growth dimension l as a function of $\phi$ for $n=1000$ and $c_{f r} \cdot \Delta \gamma \cdot l_{\text {slide }}=1.1 \cdot 10^{-6} \mathrm{~m}^{-1}$.

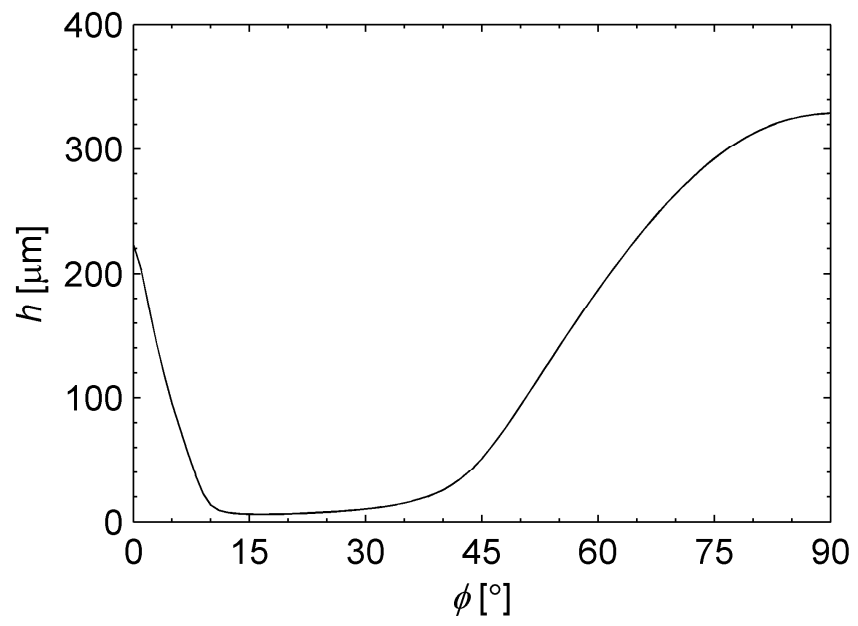

Figure 4.36. Lump growth dimension $h$ as a function of $\phi$ for $n=1000$ and $c_{f r} \cdot \Delta \gamma \cdot l_{\text {slide }}=1.1 \cdot 10^{-6} \mathrm{~m}^{-1}$.

In the figures 4.37 and 4.38 the effect of ellipticity is shown. The effect of ellipticity is strong for the case that $a / b<5$. In that range the asperity changes from a symmetrically 
loaded body to a strong asymmetrically loaded body. For increasing values of $a / b$ the lump growth goes very slowly to the state of asperities with infinite width. The lump growth is based on the assumption that the lump forms one body that is internally well connected and that an ellipsoid can still be used to fit the lump. For large values of $a / b$, in fact, the asperity has a body with $2 \mathrm{D}$ characteristics with an orientation $\phi$. So, using the ellipse fit for large values of $a / b$ is questionable.

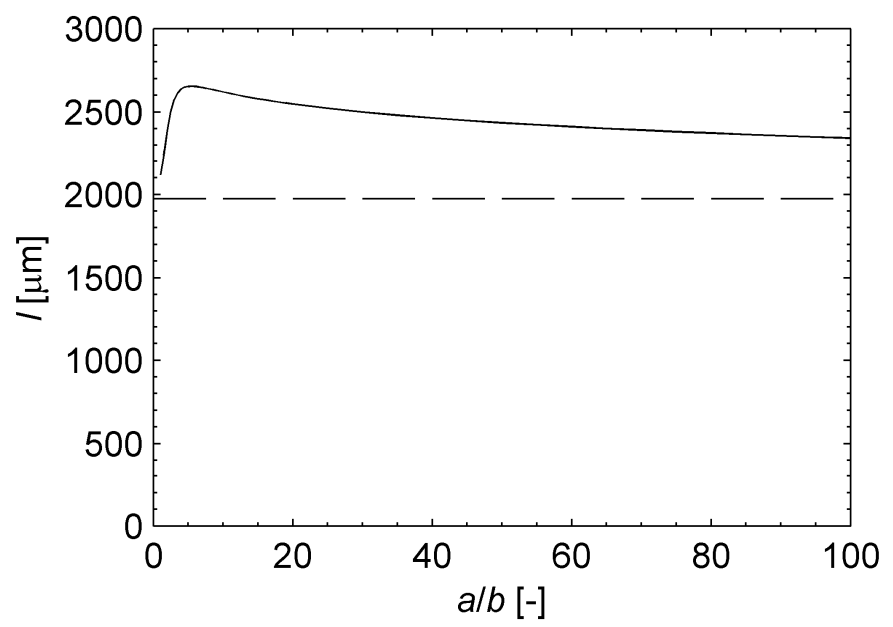

Figure 4.37. Lump growth dimension $l$ as a function of $a / b$ for $n=1000, \quad c_{f r} \cdot \Delta \gamma \cdot l_{\text {slide }}=1.1 \cdot 10^{-6} \mathrm{~m}^{-1}, \quad b=0.5 \mu \mathrm{m}$ and $\phi=45^{\circ}$. The dashed line is the asymptote of $a / b \rightarrow \infty$. 


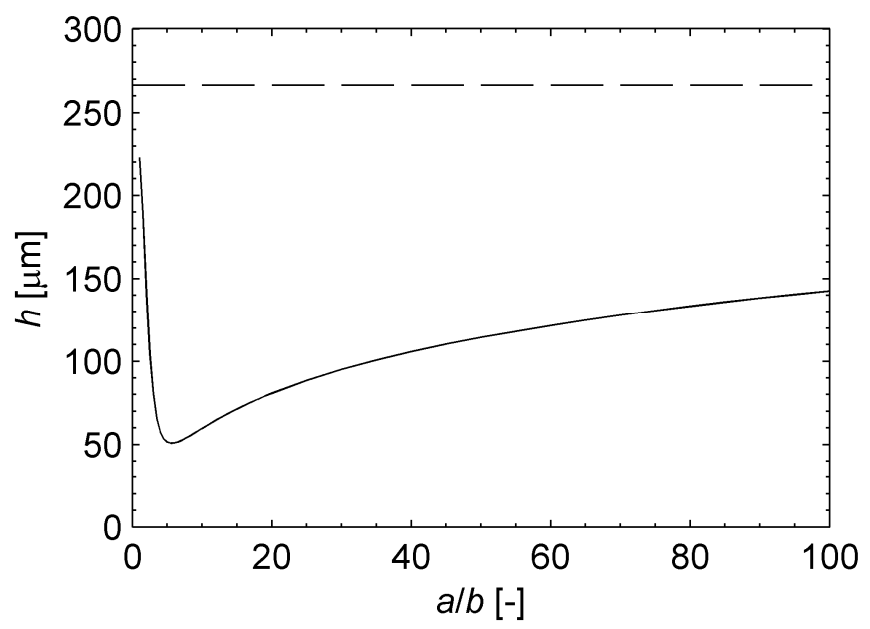

Figure 4.38. Lump growth dimension $h$ as a function of $a / b$ for $n=1000, \quad c_{f r} \cdot \Delta \gamma \cdot l_{\text {slide }}=1.1 \cdot 10^{-6} \mathrm{~m}^{-1}, \quad b=0.5 \mu \mathrm{m}$ and $\phi=45^{\circ}$. The dashed line is the asymptote of $a / b \rightarrow \infty$.

The last effect that will be shown is the effect of scale. In the results shown before, $b=0.5$ $\mu \mathrm{m}$ was used. In the figures 4.39 and 4.40 results of $b=0.5 \mu \mathrm{m}$ are compared with results of $b=5 \mu \mathrm{m}$. The scale effect is shown for two different ellipticities: $a / b=1.5$ and $a / b=5$. For the case $a / b=1.5$ no strong scale effect can be observed. The graphs of $l$ are hardly distinguishable. The graphs of $h$ show a small difference, it grows with the same velocity, only it is slightly delayed. For case $a / b=5$ the differences are slightly larger. Specially for the growth in height. But, compared with the differences in starting geometry dimensions (a factor 10) the differences in height are relatively small.

The small differences in dimensions after material transfer can be explained by the fact that the deposited layer is in both cases the same. The geometry shape determines the thickness of the layer, not the dimensions. As can be seen in figure 4.40 the large asperity needs more production steps to fill the 'gap' in front of the asperity, before it grows in height. So, in the beginning steps of lump growth, the differences between small and large asperities are reduced already. 


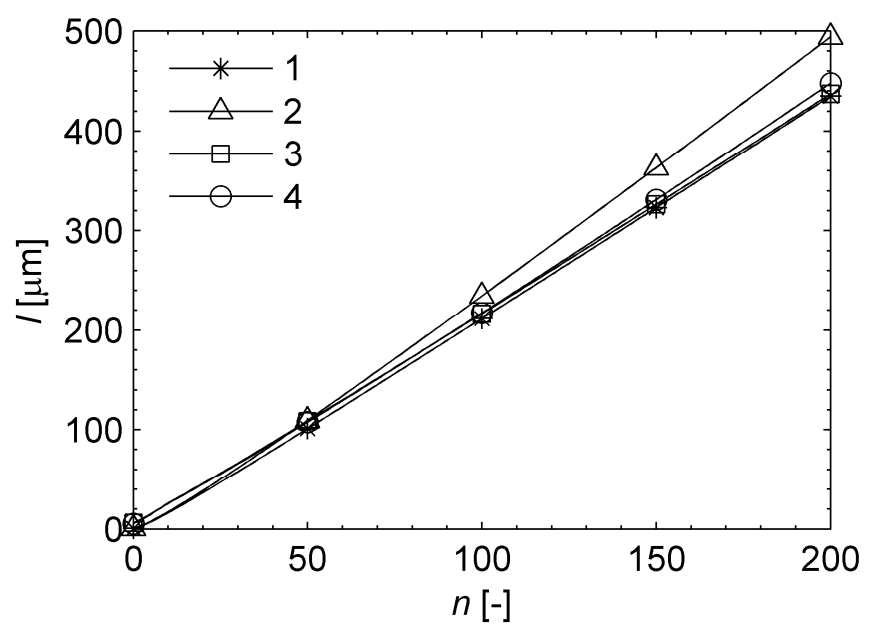

Figure 4.39. Lump growth dimension $l$ as a function of $n$ for different sizes of the asperity. For graphs 1 and $2 b=0.5 \mu \mathrm{m}$; for graphs 3 and $4 b=5 \mu \mathrm{m}$. For graphs 1 and $3 a=1.5 b$; for graphs 2 and $4 a=5 b$. Other values are $c_{f r} \cdot \Delta \gamma \cdot l_{\text {slide }}=1.1 \cdot 10^{-6} \mathrm{~m}^{-1}, \phi=45^{\circ}$ and $h=0.6 \mathrm{~b}$.

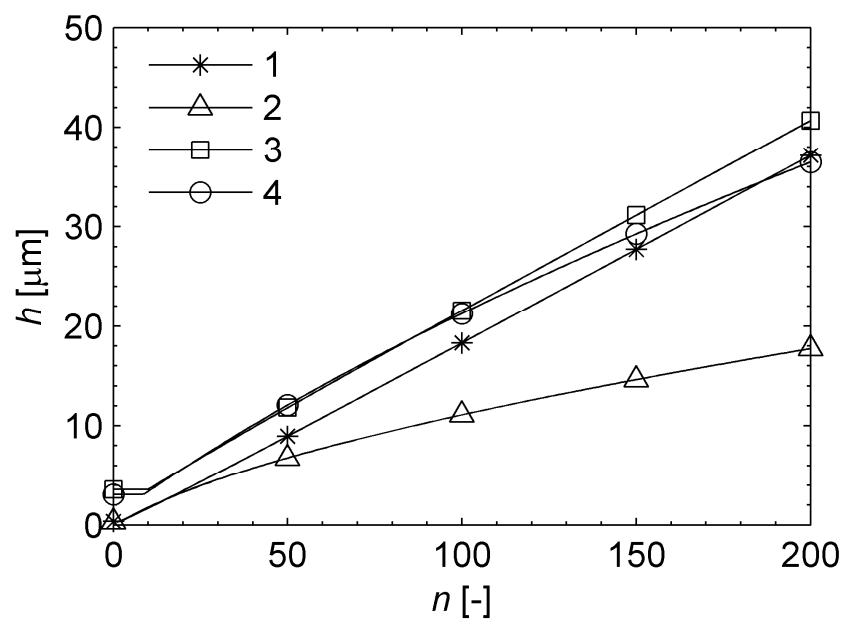

Figure 4.40. Lump growth dimension $h$ as a function of $n$ for different sizes of the asperity. Properties are the same as given in figure 4.39. 


\subsection{Summary}

A single asperity lump growth model is developed. This model is based on observations of the experiments in chapter 3 . In these experiments, material transfer takes place from the sheet material to the scratch pin of tool material. On the pin a lump is formed with a new attack angle. The model considers galling as a process that is based on two stages. In the first stage material is transferred from the sheet to the tool, which forms a transfer layer on the asperity. In the second stage, a part of the transferred layer shears off, when the asperity with lump is not strong enough to resist the contact forces. When in the lump the mechanical stresses exceed the material strength, a part of the lump shears from it, in order to get a mechanical stable stress state within the lump. In this way, the model is able to explain the formation of a new attack angle, dependent on the strength of the lump.

From the parameter study performed with the developed lump growth model some conclusions can be drawn.

- The factors $c_{f r} \cdot \Delta \gamma \cdot l_{s l i d e}$ and $n$ are of great influence. In fact, the factor formed by the multiplication $c_{f r} \cdot \Delta \gamma \cdot l_{s l i d e} \cdot n$ gives an important factor of the lump growth. A larger factor gives a faster lump growth.

- Lump growth depends on the orientation $\phi$ of the asperities. The best orientation of the asperity, to reduce the lump growth, would lie in the range $10^{\circ}<\phi<45^{\circ}$. For these orientations the lump has a strong net load in $y$-direction, which limits the lump growth in height. A wide range for $\phi$ is given with a relatively low lump growth, but if $\phi$ increases, the attack angle also increases. An increasing attack angle results in a larger wear volume, which result in more pollution of the tooling. So, minimizing both, the lump growth and the wear volume, $\phi$ should be around $10^{\circ}$.

- Differences in the ratio $a / b$ have a strong effect on lump growth for smaller ratios $(a / b<5)$. Here, also the net load in $y$-direction increases when $a / b$ goes from 1 to 5 , because of the increasing asymmetry.

- Up or down scaling of the asperity has a minor effect when the ratio between the dimensions remains the same.

In the cases that are shown of the parameter study, the asperities are in all cases too high to form immediately a stable lump. So, $n$ has to exceed a certain number before the lump grows in height. In the case which started with an asperity that is flatter than the as few as possible stable case, the wear rate would be lower and therefore less wear material that can be deposited on the tool surface is available.

For production practice, the number of products $n$ that can be formed between maintenance stops, should be maximized. That means that the factor $c_{f r} \cdot \Delta \gamma \cdot l_{\text {slide }}$ has to be reduced. The value of $l_{\text {slide }}$ is determined by the product, maybe the production process can be changed a 
bit, but in general this is not the parameter to gain real profit. Assuming that $c_{f r}$ is a constant that cannot be changed, the value of $\Delta \gamma$ should be reduced. In the sense of the bulk material of the product, this is difficult to realize, but the value of $\Delta \gamma$ can be reduced by 'polluting' the surface with a proper lubricant. Before the first layer is deposited on the tool, $\Delta \gamma$ is formed by the couple tool material and workpiece material. This is a factor that can be used to reduce the material transfer initialization. Another strategy to maximize production is grinding the tool surface in a proper manner. One aspect is the attack angle that is created in this manner. A small attack angle results in a small wear rate and thereby in a small amount of wear volume. Another aspect is the direction of the grinding scratches, because this direction determines the orientation of the tool asperities that come in contact with workpiece material. The grinding scratches should be more or less in line with the sliding direction (with some misalignment, $\phi=10^{\circ}$ ) of the workpiece material over the tool.

From the single asperity galling model, the influences of different parameters on a single asperity are known. In chapter 5 a multi asperity model will be formulated, in which operational condition are included, to predict galling in real forming processes. 


\section{Lump growth multi asperity contact}

\subsection{Introduction}

A surface consists of a lot of asperities. In the former chapter a model is derived for a single asperity. In this chapter, the model will be implemented for a contact situation of multiple asperities. The first step is determining the asperities in contact. A transformation has to be made from raw measurement data to a set of asperities that are suitable for the galling model. The asperities will be described as paraboloids with an elliptical base. This transformation will be presented in section 5.2.

The galling model that is presented in chapter 4 is based on polyhedron shaped asperities with a hexagon base. This hexagon base will be constructed from the elliptical base as discussed in section 5.2. The construction of the hexagon base from the elliptical base is discussed in section 5.3. The shape of the hexagon is dependent on the scratch direction, because it has vertices that are aligned with the sliding direction. In section 5.4 the effect of galling is calculated with the set of hexagon based asperities in sliding contact with a sheet material.

A validation of the model with experiments is presented in section 5.5. The development of the Galling Performance Indicator and its implementation as a post processor for deep drawing simulations is discussed in section 5.6.

\subsection{Contact of elliptic asperities}

For the implementation of contact and wear models on real surfaces the surfaces have to be described in a certain manner. The first step is the measurement of the surfaces. The results of these measurements will be stored as discretized height data matrices, as is described in section 2.2.3. In a lot of situations the height data matrices have to be processed further to make it suitable for a model. The steps that are described in this section are the contact determination on the meso and micro scale and finally the creation of an elliptic paraboloid shaped asperity from the contact on micro scale.

\subsubsection{Meso scale}

The contact on the meso scale will be determined as is described in section 2.2.2. The relations (2.2) and (2.3) will be adapted to guarantee force equilibrium. In section 2.2.2 it has been assumed that in the formed plateaus full contact exists and the contact pressure equals the hardness. In general, these conditions are not satisfied with the contact model of the micro scale as given in section 2.2.3. Further, during relative motion of the contact surfaces with plastic sheet material, full contact in the plateaus is not possible, because only 
the frontal half of the asperities is in contact. Therefore, in relation (2.2), $\alpha$ will be split in a meso part $\alpha_{\text {meso }}$ and a micro part $\alpha_{\text {micro }}$, which results in the following relation:

$$
p_{a}=\alpha_{\text {meso }} \alpha_{\text {micro }} H
$$

In relation (5.1) $\alpha_{\text {meso }}$ is defined as $A_{\text {plateaus }} / A_{n}$ and $\alpha_{\text {micro }}$ defined as $A_{r} / A_{\text {plateaus }}$ with $A_{\text {plateaus }}$ the area of the plateaus. Relation (2.3) will be adapted for calculating $\alpha_{\text {meso }}$ :

$$
\alpha_{\text {meso }}=\frac{A_{\text {plateaus }}}{A_{n}}=\int_{h_{z}}^{\infty} \phi_{\text {sheet }}(z) d z
$$

Relation (5.2) assumed a smooth tool surface. In the case where the tool surface is relatively smooth compared to the sheet surface, this gives a good approximation of the formed plateaus. Typically, the $R_{q}$-value of the tool is a number of times smaller than that of the sheet. Sheet materials have mostly an $R_{q}$-value of $1-2 \mu \mathrm{m}$, see for example section 3.2.2, tool surfaces have mostly an $R_{q}$-value of about 0.25 to $0.5 \mu \mathrm{m}$. When the surfaces have a Gaussian height distribution, the influence of the tool roughness on $\alpha_{\text {meso }}$ is already negligible when the roughness of the sheet is three times higher, which can be explained as follows. Bringing two surfaces into contact means that the local separation of these surfaces equals the subtraction of the individual local surface heights. Addition and subtraction of Gaussian distributions results again in a Gaussian distribution in which variance $\left(R_{q}{ }^{2}\right)$ is the summation of the variances of the original distributions. The $R_{q}$-value of a surface equals the standard deviation of the height distribution. So, if the $R_{q}$-value of the sheet is three times higher than the $R_{q}$-value of the tool, the effect of the variance of the tool is only 10 percent on the subtracted surfaces. This means 5 percent on the standard deviation.

To determine the contact area on the meso scale, relation (5.2) has to be solved as an implicit relation, because $h_{z}$ is not known beforehand. The sheet surface above the level $h_{z}$ forms the contact on the meso scale. When $p_{a}$ and $H$ are known, $\alpha$ follows from relation (2.2). However, $\alpha$ is split in $\alpha_{\text {meso }}$ and $\alpha_{\text {micro }}$, so an assumption will be made about this split. A contact between a rigid tool and a plastic sheet with a relative sliding motion should typically result in $\alpha_{\text {micro }}$ with a value of 0.5 , because only the frontal half of sliding asperities are in contact. For special cases, like a tool with a saw tooth surface profile, the value of $\alpha_{\text {micro }}$ can exceed this value. In the calculations in this chapter, the value of $\alpha_{\text {micro }}$ is assumed to be 0.5 . In the explanation of this assumption, two issues have to be taken into account. The formation of plateaus is a plastic deformation process, so high pressures are needed, in the order of the hardness. But, as explained above, during sliding, in combination with most tool surfaces, only half of the plateau surface can be in contact. In order to have an as high as possible pressure as well as half of the plateaus in contact $\alpha_{\text {micro }}=0.5$ is required. From this assumption, it follows that $\alpha_{\text {meso }}=2 \alpha$. 


\subsubsection{Micro scale}

On the micro scale, the contact will be determined on the plateaus that are formed on the meso scale, as presented in section 5.2.1. The contact will be described as a contact between rigid elliptical paraboloid tool asperities in ideal plastic sheet material. The plateaus are assumed to be flat, so the tool asperities come into contact with a flat plane. The description of a surface by elliptic paraboloid shaped asperities means a simplification of the real surface. The roughness measurement that is stored in a matrix with height data consists of the real surface within a certain frequency range in the spatial domain. The minimal spatial frequency is determined by the dimensions of the measurement area, the maximum spatial frequency by the spatial resolution. Within this range the data set is an exact representation of the surface, leaving measurement errors out of consideration. Using the description of the surface by a set of elliptic paraboloid shaped asperities reduced the amount of data enormously, because a total description of the surface is replaced by a set of asperities that are given by a number of parameters like the radii, the orientation and location. Further, the description is dependent on the contact conditions. The number and the size of the asperities are related to the contact situation, as is shown in figure 5.1.
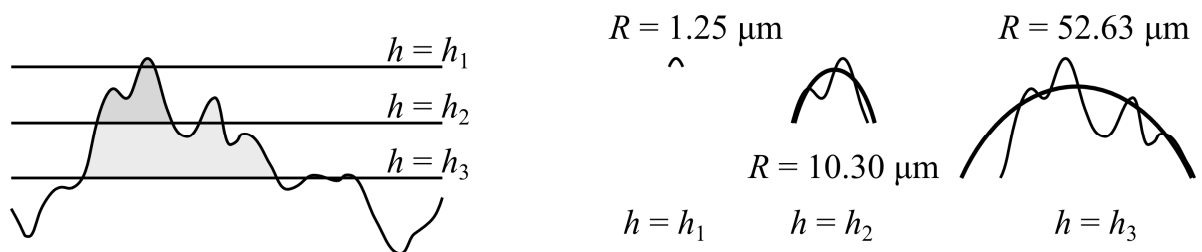

Figure 5.1. Asperity definition at different surface separations, after [36].

As a basis of the determination of the asperities, the approach of Masen [36] will be used. Here, a contact model using a deterministic approach is presented. The input variables are two contact bodies with their surface geometry and their relevant physical properties for contact like the modulus of elasticity and hardness. In [36] one of the surfaces is assumed to be flat. Further, the normal force is needed to calculate the separation and related to this the contact spots. The contact is determined by using the condition of force equilibrium, as given in the following equation:

$$
F_{n}=\sum_{i} F_{n i}
$$

In equation (5.3), the general part of equation (2.6) is repeated. Usually, the force $F_{n}$ is the known quantity and the values of $F_{n i}$ are the unknowns. The values of $F_{n i}$ are unknowns in two ways. The contact force of an individual contact spot is unknown, but also the number and shape of these contact spots. That the number of contact spots changes during loading a 
surface can be seen in figure 5.1. At the separation given by $h_{1}$ there is one contact spot. Increasing the load that leads to a separation of $h_{2}$, two spots are in contact. Halfway $h_{2}$ and $h_{3}$ both contact spots are merged. For every separation, the definition of the asperity belonging to a contact spot changes as is shown at the right-hand side of the figure. In general, equation (5.3) can be solved by iterating over the separation.

The contact force of an individual contact spot depends on the material properties and the surface geometries in combination with the separation. In the case where a rigid rough surface comes in contact with an ideal plastic flat surface, solving equation (5.3) can be simplified. Assuming that the contact pressure in the real contact area equals the hardness, the total real contact area is already known as the normal force divided by the hardness. The contact spots are found by the separation that causes an intersection area equal to the real contact area. This can be done using the following relation:

$$
\alpha_{\text {micro }}=\frac{A_{r}}{A_{\text {plateaus }}}=\int_{h_{z}}^{\infty} \phi_{\text {tool_on_plateaus }}(z) d z
$$

The function $\phi_{\text {tool_on_plateaus }}$ is the height density function of the tool surface in plateau regions. Relation (5.4) has to be solved like relation (5.2) to find the contact spots on the plateaus.

The contact according to relation (5.4) is determined for the static case. During a sliding contact, only the frontal part of the asperities is in contact. To satisfy normal force equilibrium, the asperities have to penetrate further into the sheet plateaus. This will be done with the set of asperities defined for the static case, so no new set of asperities will be defined for the sliding contact.

The strategy to determine the contact spots on the plateaus has some consequences for the determination of asperities. Only asperities or the parts from an asperity within the plateaus are taken into account. So, an asperity determined according to this method can have smaller dimensions than the real asperity. In the case of very long asperities, like on grinded tool surfaces, it can be in contact with more than one plateau. Every separate contact spot of the real asperity forms a separate model asperity. So, one real asperity can be split into more model asperities. Parts of the tool outside the plateaus are neglected.

Once the contact spots are known, an elliptic paraboloid is fitted through the height data belonging to the contact spot. Two constraints will be given: the contact area and the volume enclosed by the separation plane should be equal for the real surface geometry and the paraboloid [35]. The first constraint results in an equal hardness, namely the normal force is carried by the same contact area. The second constraint results in the same work of penetration, namely the hardness times the volume of the moved material. To define the paraboloid, the unknowns are the major and the minor radius of the ellipse $a$ and $b$ at the separation plane, the orientation $\phi$ and the height $s$. The definition of $a, b$ and $\phi$ are given in 
figure 5.2. The values of these quantities are calculated using the second moments of area with respect to the centroid of a contact spot. Using $n^{\text {th }}$ order moments of area for pattern recognition in digital images is a concept published by $\mathrm{Hu}$ [25]. Chaudhuri [12] uses the second moments of area with respect to the centroid for fitting an ellipse through a $2 \mathrm{D}$ object. The contact spots and their properties are determined using a discretized height data map, so the integrals given below can be written as a summation. The following relations are used:

$$
\begin{gathered}
I_{x x}=\int\left(y-y_{c}\right)^{2} d A=\Delta x \Delta y\left(\frac{n(\Delta y)^{2}}{12}+\sum_{i=1}^{n}\left(y_{i}-y_{c}\right)^{2}\right) \\
I_{y y}=\int\left(x-x_{c}\right)^{2} d A=\Delta x \Delta y\left(\frac{n(\Delta x)^{2}}{12}+\sum_{i=1}^{n}\left(x_{i}-x_{c}\right)^{2}\right) \\
I_{x y}=\int\left(x-x_{c}\right)\left(y-y_{c}\right) d A=\Delta x \Delta y \sum_{i}\left(x_{i}-x_{c}\right)\left(y_{i}-y_{c}\right)
\end{gathered}
$$

In the relations above $\Delta x$ and $\Delta y$ are the distances in $x$ and $y$-direction between measurement points on the surface, $x_{c}$ and $y_{c}$ are the coordinates of the centroid of the contact spot, $x_{i}$ and $y_{i}$ are the coordinates of measurement point $i$ from the set of $n$ points that forms the total contact spot. The terms $(\Delta x)^{2} / 12$ and $(\Delta y)^{2} / 12$ give the second area moment of a single data point with respect to its own centroid using an area of $\Delta x$ times $\Delta y$. In [12] these terms are omitted and only the displacement parts, like $x_{i}-x_{c}$, are used from the parallel axis theorem of Steiner. In the calculations presented in this chapter the equations (5.5a) to (5.5c) will be used. For contact spots that consist of very few data points or are very narrow in one direction, the extra term makes sense. 


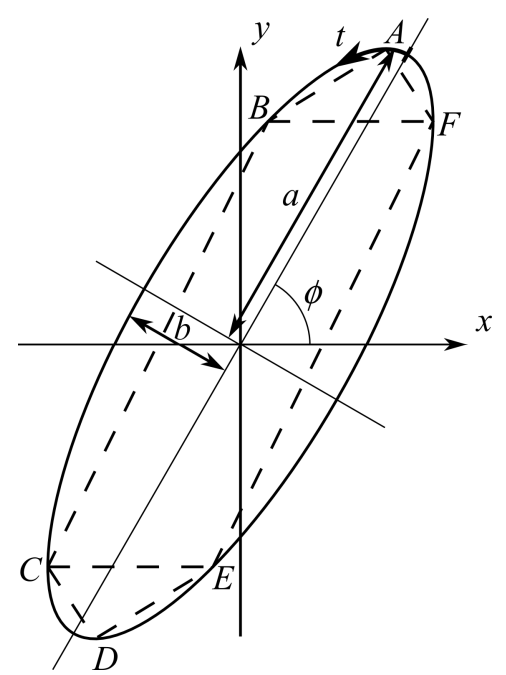

Figure 5.2. Elliptic base of an asperity and its transformation to a hexagon base.

There exists a unique ellipse that has the same second moments of area with respect to the centroid as calculated with the equations (5.5a) to (5.5c). This ellipse will be determined and scaled, so the contact size of the paraboloid will be equal to the real contact spot. Using Mohr's circle it follows that:

$$
\begin{gathered}
I_{\max }=\frac{I_{x x}+I_{y y}}{2}+\sqrt{\frac{1}{4}\left(I_{x x}-I_{y y}\right)^{2}+I_{x y}^{2}} \\
I_{\text {min }}=\frac{I_{x x}+I_{y y}}{2}-\sqrt{\frac{1}{4}\left(I_{x x}-I_{y y}\right)^{2}+I_{x y}^{2}} \\
\phi=\frac{1}{2} \operatorname{atan} 2\left(2 I_{x y}, I_{y y}-I_{x x}\right)
\end{gathered}
$$

In equation (5.6c) atan2 $(y, x)$ is the four-quadrant extension of the arctangent that gives the angle between point $(x, y)$ and the positive $x$-axis. In this manner the range of the arctangent is extended from $|\phi| \leq 0.5 \pi$ to the needed $|\phi| \leq \pi$.

The ellipse that has to be determined has the following second moments of area:

$$
\begin{aligned}
& I_{\text {max }}=\frac{\pi a^{3} b}{4} \\
& I_{\text {min }}=\frac{\pi a b^{3}}{4}
\end{aligned}
$$


The values of $a$ and $b$ can be immediately solved by making $I_{\max }$ of equation (5.7a) equal to the one of equation (5.6a) and $I_{\min }$ of equation (5.7b) equal to the one of equation (5.6b). Due to the final scaling of the ellipse to get equal sizes of the real contact spot and the ellipse contact, the ratio $a$ and $b$ is of more importance, which is given by:

$$
r=\frac{b}{a}=\sqrt{\frac{I_{\min }}{I_{\max }}}
$$

So:

$$
a=\sqrt{\frac{A}{\pi r}}
$$

From equations (5.6c), (5.8) and (5.9) the values of $a, b$ and $\phi$ can be calculated. The height $s$ will be defined as given in figure 2.8. In this figure $h$ is the separation between the nominal surfaces of the contact bodies and $s$ the height of a single asperity. So, the penetration of a rigid asperity in the ideal plastic body is given by $s-h$. The penetration can be determined by the constraint of volume conservation. This results in:

$$
s=h+\frac{2 V}{\pi a b}
$$

In equation (5.10) is $V$ the volume of asperity above the $z$-coordinates $h$ which can be determined using a summation of the height data $s_{i}$ from the height data matrix:

$$
V=\Delta x \Delta y \sum_{i=1}^{n}\left(s_{i}-h\right)
$$

The major and minor radius of the asperity surface $R_{a}$ and $R_{b}$ are given by:

$$
\begin{gathered}
R_{a}=\frac{a^{2}}{2(s-h)} \\
R_{b}=\frac{b^{2}}{2(s-h)}=r^{2} R_{a}
\end{gathered}
$$

Using the strategy as given above, all the information of an asperity is obtained. Its in-plane location is the centroid of the contact spot. Other parameters that can be determined are its height $h$, major and minor radius $R_{a}$ and $R_{b}$ and its orientation $\phi$.

\subsection{Conversion from ellipse to hexagon based asperity}

For the galling model that is presented in chapter 4 a polyhedron shaped asperity with a hexagon base is needed. The construction of this asperity will be presented in this section. 
Starting point is the geometry of an ellipse, as given in figure 5.2 and calculated in section 5.2. An ellipse is defined by its two main radii, the orientation and the position on a plane. Here, the origin of the coordinate axes is positioned on the centre of the ellipse. The $x$-axis points in sliding direction, the $z$-axis is perpendicular on the nominal surface. The $y$-axis is perpendicular on the other two coordinate axes. The major radius has length $a$, the minor radius has length $b$. The orientation will be given by $\phi$, the angle between the major axis of the ellipse and the $x$-axis. The value of $\phi$ can differ from the $\phi$ as calculated in section 5.2 in the case where the height measurement is not aligned with the sliding direction. The domain of $\phi$ is limited by $-0.5 \pi<\phi \leq 0.5 \pi$, because of symmetry and to prevent multiple definitions of one ellipse.

The hexagon is constructed by constructing the elliptic base in this coordinate system. The extremes in $y$-direction form the first two points. The next two points are the extremes in $x$-direction. The last two points are found by finding the two other points with the same $y$-coordinates as the former points. The points that are found on the ellipse are given in figure 5.2 by the dashed hexagon $A B C D E F$. The next step is to scale the hexagon, so the hexagon gets the same surface area as the ellipse.

The dimensions of the hexagon that will be used in the galling model are given in figure 5.3. In this figure, the hexagon has more degrees of freedom than follows from the ellipse as given in 5.2. In the case of elliptical asperities it is assumed that an asperity can have an orientation, but that it has comparable dimensions at both sides of the major axis. Due to galling, material is deposited at one side, so a kind of symmetry will be gone. Compared with the hexagon as given in figure 5.2, the number of degrees of freedom should be reduced. In order to do this, the lengths in $x$-direction $l$ and $l_{b}$ are taken as equal in the ellipse just as $w_{\mathrm{I}}$ and $w_{\text {III. }}$. 


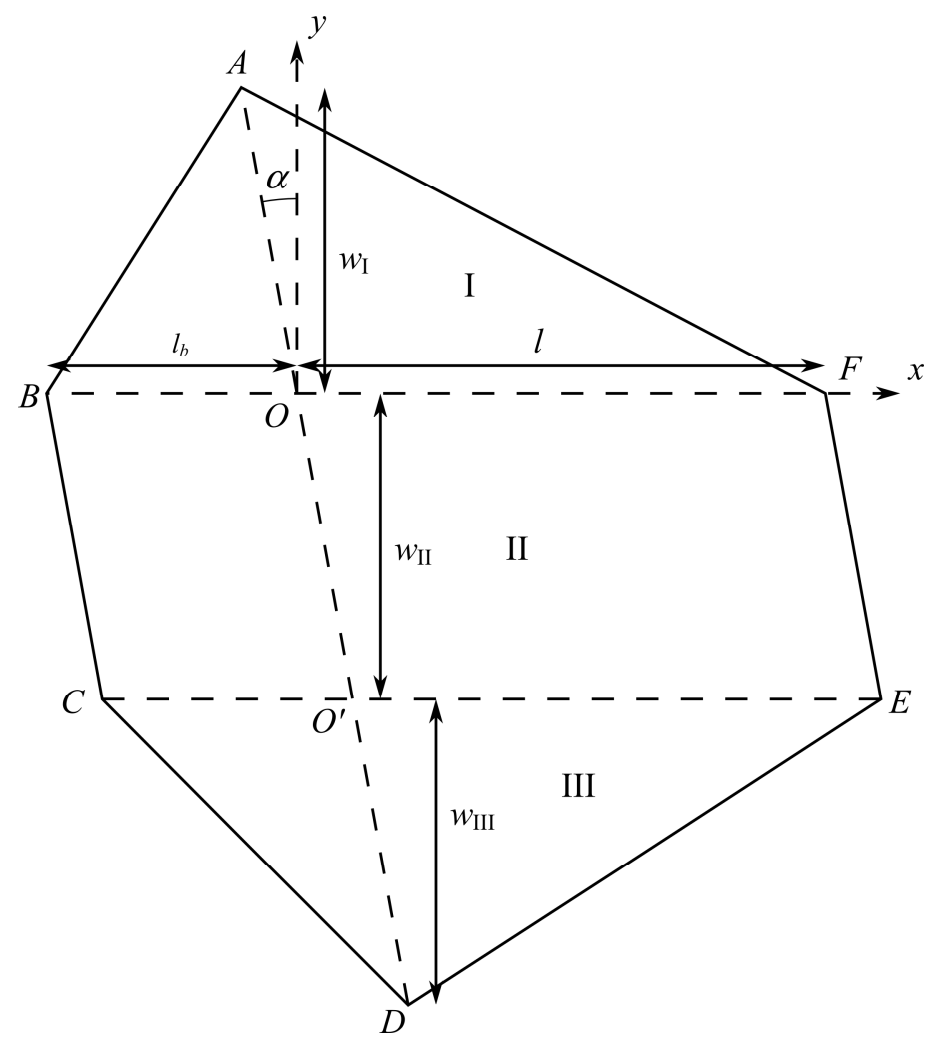

Figure 5.3. Dimensions of the hexagon base.

The ellipse as shown in figure 5.2 can be given, in a parametric form with parameter $t$, as:

$$
\begin{aligned}
& x=a \cos \phi \cos t-b \sin \phi \sin t \\
& y=a \sin \phi \cos t+b \cos \phi \sin t
\end{aligned}
$$

The six vertices of the hexagon are determined, using equations (5.13a) and (5.13b). These points will first be derived for the case that $0<\phi<0.5 \pi$. Point $F$ is defined by the location on the ellipse with the maximum $x$-coordinate. The value of $t_{F}$ of this point is given by:

$$
t_{F}=-\operatorname{atan}\left(\frac{b}{a} \tan \phi\right)
$$

The subscript that is used for $t$ gives the vertex to which the value of $t$ belongs. In the following part, these subscripts will also be used for the $x$ and $y$-coordinates.

Point $A$ is defined by the location on the ellipse with the maximum $y$-coordinate. The value of $t_{A}$ of this point is given by: 


$$
t_{A}=\operatorname{atan}\left(\frac{b}{a} \cot \phi\right)
$$

Relations (5.14) and (5.15) can be found by the derivatives of the equations (5.13a) and (5.13b) with respect to $t$. On the extremes, one of derivatives is zero.

Point $B$ is the point on the ellipse that has the same $y$-coordinate as $F$, but is on the other side of the major axis of the ellipse. To find $t_{B}$ equation $(5.13 \mathrm{~b})$ is rewritten to:

$$
y=\sqrt{a^{2} \sin \phi+b^{2} \cos \phi} \cos \left(t-t_{A}\right)
$$

Using the symmetry of the cosine and that $y_{B}$ equals to $y_{F}$, it follows from equation (5.16) that:

$$
t_{B}-t_{A}=-\left(t_{F}-t_{A}\right) \Rightarrow t_{B}=2 t_{A}-t_{F}
$$

The coordinates of $F, A$ and $B$ are found by using the results of relations (5.14), (5.15) and (5.17) in equations (5.13a) and (5.13b). The coordinates of $C, D$ and $F$ are given by:

$$
\begin{aligned}
& \left(x_{C}, y_{C}\right)=-\left(x_{F}, y_{F}\right) \\
& \left(x_{D}, y_{D}\right)=-\left(x_{A}, y_{A}\right) \\
& \left(x_{E}, y_{E}\right)=-\left(x_{B}, y_{B}\right)
\end{aligned}
$$

For the case that $-0.5 \pi<\phi<0$ the relations (5.14) to (5.17) cannot be used immediately. Some fundamental properties of the combination of the hexagon and the ellipse change. Vertex $F$ loses its extreme $x$-coordinate and $E$ gets it. The same change happens for vertices $C$ and $B$. The following relation can be derived for this situation:

$$
\begin{gathered}
t_{E}=-\operatorname{atan}\left(\frac{b}{a} \tan \phi\right) \\
t_{D}=\operatorname{atan}\left(\frac{b}{a} \cot \phi\right) \\
t_{C}=2 t_{D}-t_{E}
\end{gathered}
$$

The relations (5.13a) and (5.13b) and (5.18a) to (5.18c) remain valid for $-0.5 \pi<\phi<0$.

There are a few special cases where the hexagon reduces to a quadrilateral:

- The radii $a$ and $b$ are equal. The hexagon base reduces to a square with two vertices on the $x$-axis and two vertices on the $y$-axis.

- The orientation of $\phi$ is 0 . The hexagon base reduces to a rhombus, with the vertices determined by the major radius on the $x$-axis and the vertices determined by the minor radius on the $y$-axis. 
- The orientation of $\phi$ is $0.5 \pi$. The hexagon base reduces to a rhombus, with the vertices determined by the minor radius on the $x$-axis and the vertices determined by the major radius on the $y$-axis.

The ellipse is used as a tool to construct the shape of the hexagon. The coordinates that are used for the ellipse are local coordinates with the origin at the centre of the ellipse. Now, the interesting dimensions of the hexagon are defined. Finally, the hexagon must have a proper surface area, so it will be scaled proportionally in $x$ and $y$-direction, that the surface area equals the surface area in the original contact spot.

The dimensions of the hexagon are determined as follows:

- The values of $w_{\mathrm{I}}$ and $w_{\text {III }}$ are equal to $y_{A}-y_{F}$.

- The value of $w_{\mathrm{II}}$ equals $y_{F}-y_{E}$, that is the same as $2 y_{F}$.

- The values of $l$ and $l_{b}$ are equal to $0.5\left(x_{F}-x_{B}\right)$.

- The value of $\alpha=-\operatorname{atan}\left(x_{A} / y_{A}\right)$.

The last step is the scaling of the hexagon. The scaling is determined by the surface area of the contact spot. The ellipse has a surface area size of $\pi a b$. The area of the hexagon is $\left(x_{F}-x_{B}\right)\left(y_{A}-y_{E}\right)$. The area of the hexagon is smaller than the ellipse, because it is an inscribed polygon of the ellipse. So scaling results in a hexagon that is larger than the one that is determined until now. The dimensions $w_{\mathrm{I}}, w_{\mathrm{III}}, l$ and $l_{b}$ will be scaled. This scaling method scales the triangles $A B F$ and $C D E$ proportionally, the parallelogram $B C E F$ scales only in $x$-direction. The scaling factor of these dimensions can be calculated using the dimensions of the original ellipse and the dimensions of the hexagon as they are determined until now by:

$$
c_{\text {scale }}=\frac{-l w_{\mathrm{II}}+\sqrt{l^{2} w_{\mathrm{II}}^{2}+\pi a b l\left(w_{\mathrm{I}}+w_{\mathrm{III}}\right)}}{l\left(w_{\mathrm{I}}+w_{\mathrm{III}}\right)}
$$

Although proportional scaling of all dimensions is an easier method, this method will not be used, because it gives bad results in the case of high values of the ratio of $a$ and $b$. This can be explained using figure 5.2 where an ellipse is shown with a ratio of $a$ and $b$ of 3 . On the $x$-axis the hexagon is much more slender than the ellipse. That means that the ellipse scales with a relatively large scaling factor. This scaling corrects the size of the area, but in absolute values the hexagon is much more scaled in $y$-direction than in the $x$-direction, which results in an asperity with a too large frontal area due to the excessive $y$-extremes. The construction of the hexagon base is described. The next step is the determination of the asperity height. The same constraints are still used as in section 5.2, that means that both the volume and the contact spot of the asperity have the same size as on the real surface, so the volume of the asperity with the hexagon base equals the volume as given in equation (5.11) also in this situation. The height is: 


$$
s=h+\frac{6 V}{\left(w_{\mathrm{I}}+3 w_{\mathrm{II}}+w_{\mathrm{III}}\right)\left(l+l_{b}\right)}
$$

The steps that are made from surface height data to the construction of polyhedron shaped asperities with a hexagon base are shown in the scheme in figure 5.4 and a graphical presentation is shown in figure 5.5. In figure 5.5, the sliding direction is not as is usual from left to right, but in the direction that is given with the small arrow $v$. With this changed direction, the lines $B F$ and $C E$ get another orientation, which means another definition of the hexagon. 


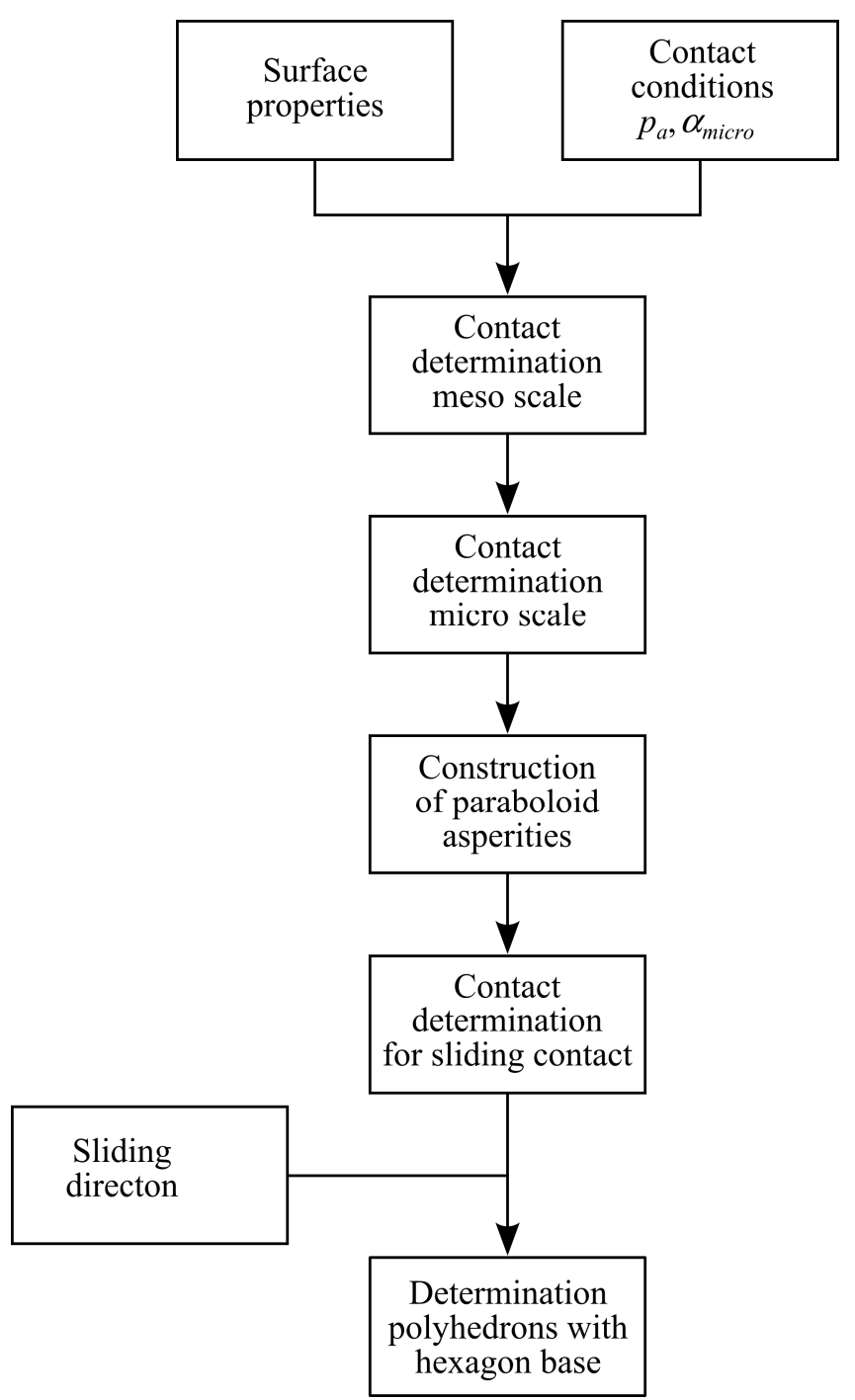

Figure 5.4. Determination of contact and polyhedrons with hexagon base. 

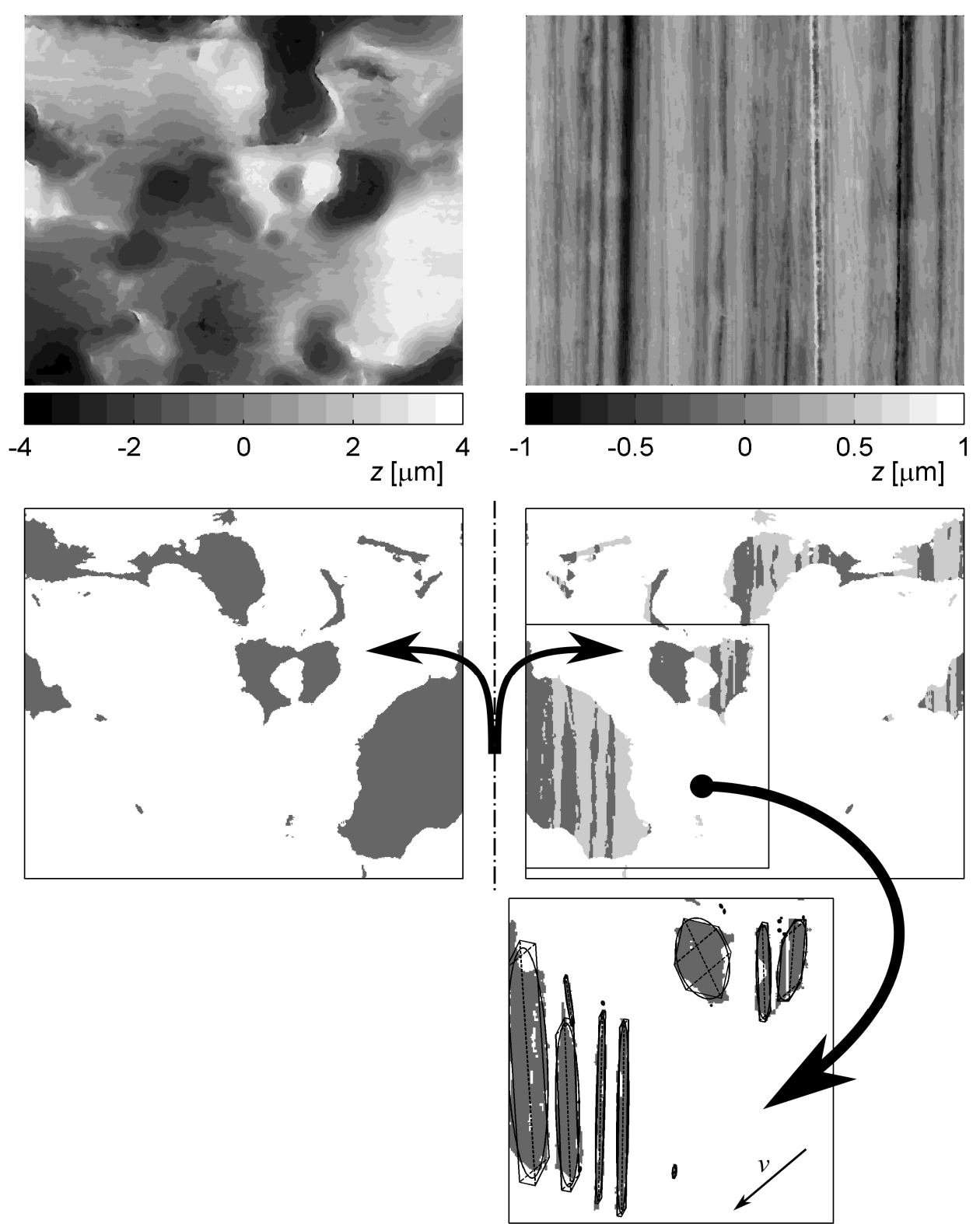

Figure 5.5. Determination of the asperities. At the top are the surface measurements $(255 \times 215 \mu \mathrm{m})$ of the sheet (left) and the tool (right). Beneath are the plateaus (left) and the contact spots of the tool with plateaus (right). The contacts are the dark grey areas on the light projections of the plateaus. At the bottom the fits are shown of the ellipses and hexagons for a part of the measured area. The arrow $v$ gives the sliding direction, which is relevant for the orientation of the hexagons. 


\subsection{Galling calculations}

\subsubsection{Calculations for a model system}

To calculate galling for the multi asperity contact between a tool and sheet surface, the single asperity growth model as developed in chapter 4 will be combined with the contact model and asperity definition as developed in the sections 5.2 and 5.3.

For combining the single asperity lump growth model with the contact model some extra steps have to be taken. After determination of the asperities the lump growth calculation has to be performed over all the asperities. According to the contact model that is used, plateaus are formed and the tool asperities are only in contact on the plateaus. That means that asperities are only a fraction of the sliding length $l_{\text {slide }}$ in contact. The fraction of plateaus on the surface is $\alpha_{\text {meso }}$, so the average contact length of an asperity reduces by the same fraction $\alpha_{\text {meso }}$ and becomes $\alpha_{\text {meso }} \cdot l_{\text {slide }}$. Before the calculation of the lump growth is performed, the separation distance is calculated from normal force balance using the contact model. Due to lump growth, the contact area increases for a given separation of the surfaces and as a result, the normal force increases. Therefore, after lump growth the separation of the surfaces has to be calculated again in order to maintain normal force equilibrium. In the lump growth model a set of hexagon based polyhedron asperities is used and with this set the separation between the surfaces will be recalculated after a lump growth step. During sliding, the area $A D E F$ as shown in figure 5.3 is in contact, so this area will be used for calculating the separation.

The implementation of the multi asperity galling model is given schematically in figure 5.6. The lump growth calculation over all the asperities is given in figure 5.6 by the block 'Determination lump growth due to galling'. This block is in fact a loop over all individual asperities.

Thermal effects as presented in section 2.3.1 are not taken into account in the calculation for reasons of clarity. It is assumed that the thermal effects are a part of the constant $c_{f r}$. In the case of a low thermal load, $c_{f r}$ will have a lower value. 


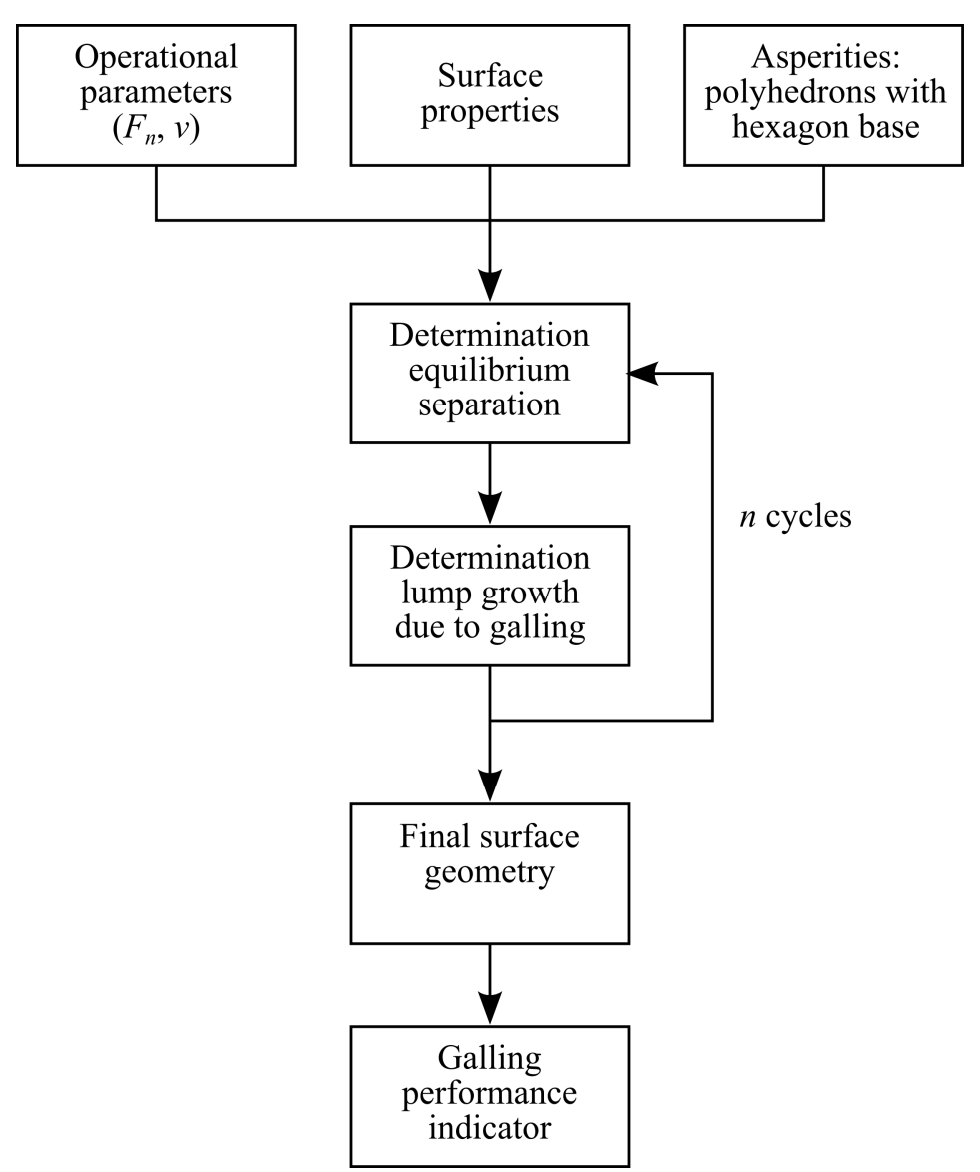

Figure 5.6. Scheme of the implementation of the multi asperity galling model.

A number of calculations are performed which will be presented in the following figures. For the calculations, a set of physical quantities is given in table 5.1. Using equation (5.1) with the data of table 5.1 it follows that $\alpha=0.2$. The used surfaces are a grinded tool surface with $R_{q}=0.23 \mu \mathrm{m}$ and a sheet material with $R_{q}=2.0 \mu \mathrm{m}$. These surfaces are shown in figure 5.5 and are actual measurements of a sheet and a tool surface. The angle between the grinding direction of the tool surface and the sliding direction that is used in the calculations is $10^{\circ}$. In figure 5.7 and 5.8 the height and the fraction of asperities in contact are shown as a function of production cycles. In figure 5.9 and 5.10 the penetration $\delta$ of every individual asperity is shown versus the total width $w_{\text {total }}$ of the scratch that it causes. Here, $w_{\text {total }}$ is defined as a sum of $w_{\mathrm{I}}, w_{\mathrm{II}}$ and $w_{\mathrm{III}}$ as shown in figure 5.3. The penetration 
equals the height of an asperity minus the separation as given in figure 5.7 by graph 1 . An average scratch depth could be calculated by the difference of graph 1 and 2 in figure 5.7 . In figure 5.9 and 5.10 only the asperities are shown that are still in contact. The asperity sets given for $n$ are $0,10,100$ and 1000. The asperities that are still in contact for $n=1000$ are marked in all the plots by asterisks.

\begin{tabular}{|l|l|l|}
\cline { 2 - 3 } \multicolumn{1}{c|}{} & Parameter & Value \\
\hline Contact parameters & $p_{a}$ & $50 \mathrm{MPa}$ \\
\cline { 2 - 3 } & $\alpha_{\text {micro }}$ & 0.5 \\
\hline Material parameters & $T$ & 0.27 \\
\cline { 2 - 3 } & $H$ & $0.5 \mathrm{GPa}$ \\
\cline { 2 - 3 } & $\Delta \gamma$ & $1.1 \mathrm{~J} \mathrm{~m}^{-2}$ \\
\hline Other parameters & $l_{\text {slide }}$ & $0.1 \mathrm{~m}^{-6} \mathrm{~m}^{2} \mathrm{~J}^{-1}$ \\
\cline { 2 - 3 } & $c_{f r}$ & $5 \cdot 10^{-}$ \\
\cline { 2 - 3 } & $\mu$ & 0.18 \\
\hline
\end{tabular}

Table 5.1. Physical parameters used in galling model.

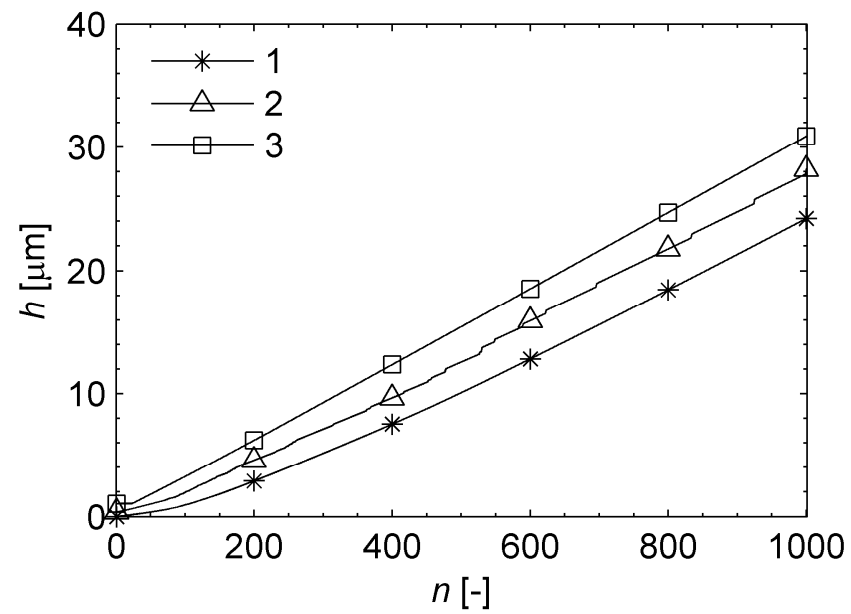

Figure 5.7. Height of the asperities as a function of the number of products $n$. Graph 1 shows the separation increase of the contact surfaces due to lump growth, graph 2 the mean height of the lumps that are still in contact and graph 3 the height of the highest lump. 


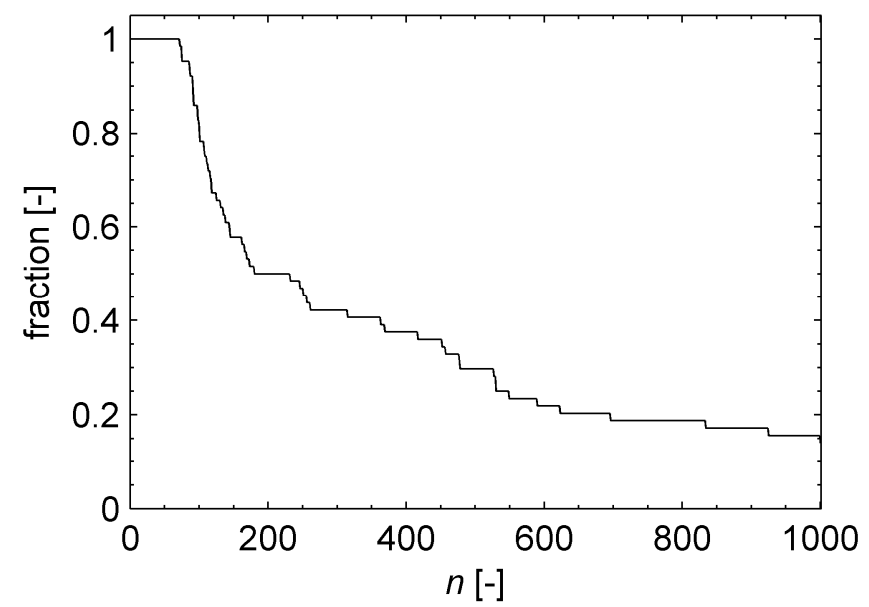

Figure 5.8. The fraction of the asperities that are still in contact as a function of $n$. 


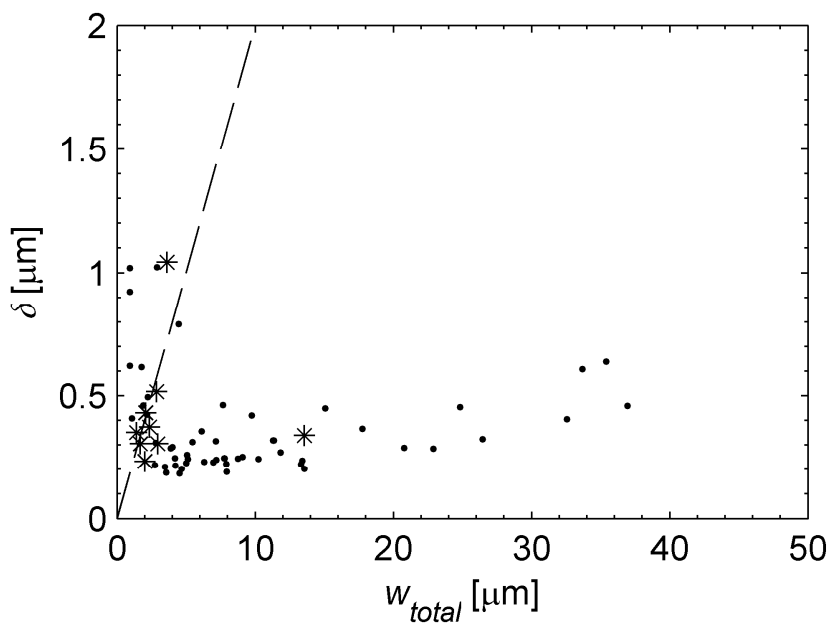

$a$

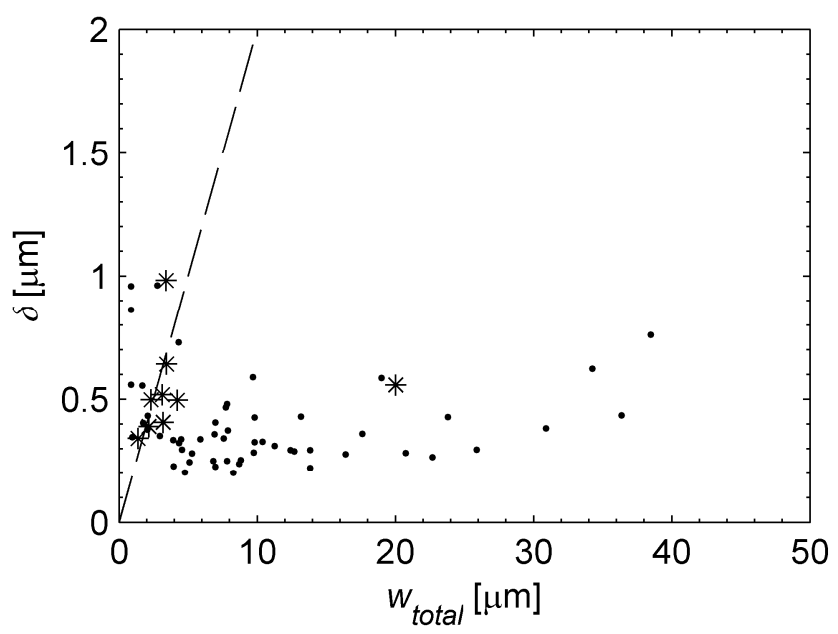

$b$

Figure 5.9. Cloud plots of the depths versus the width of the scratches from the asperities that are still in contact. It shows the start situation, $n=0$ (a) and the situation for $n=10$. The asperities that are still in contact for $n=1000$ are marked by an asterisk. 


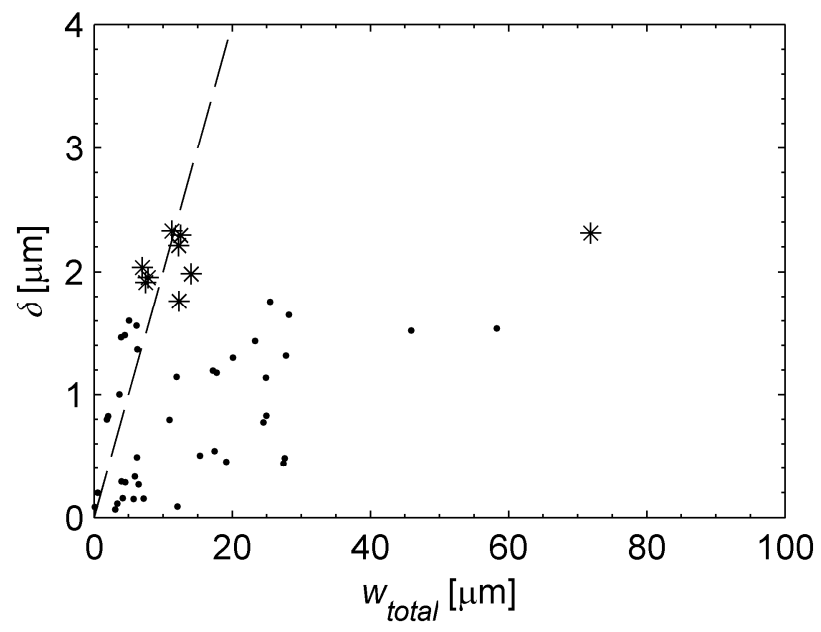

$a$

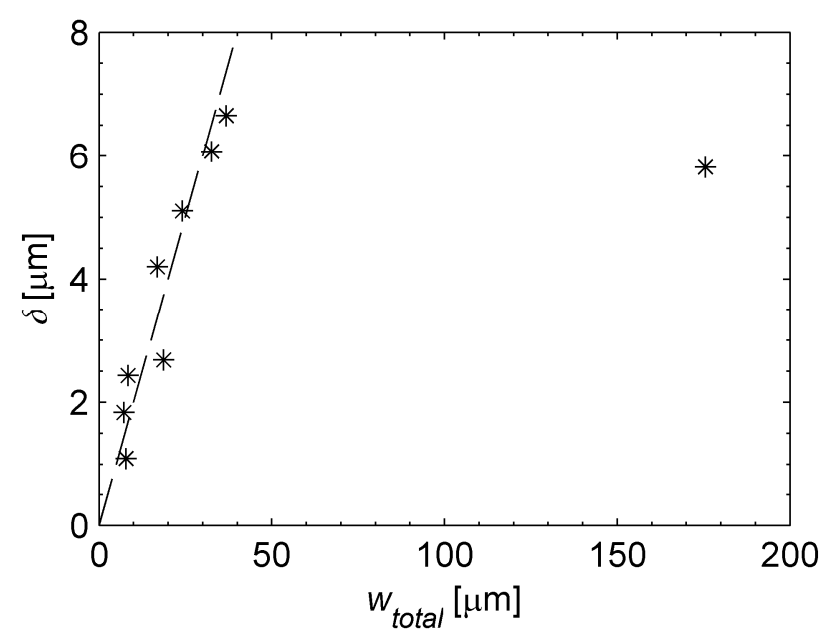

$b$

Figure 5.10. Cloud plots of the heights versus the width of the scratches from the asperities that are still in contact for $n=100$ (a) and $n=1000$ (b). The asperities that are still in contact for $n=1000$ are marked by an asterisk.

According to figure 5.7 the average lump height is almost linear as a function of the number of product cycles. For the first products, $n<100$, the graphs show a smaller amount of lump growth. According to figure 5.8, for $n<70$ all the asperities are still in contact. After $n=70$, the fraction of the asperities that are in contact decreases very fast. So, with this decreasing fraction, fewer and fewer asperities are subject to lump growth. The scratch development caused by individual lumps is shown in the figures 5.9 and 5.10 . The 
scratches caused by asperities that remain in contact at least to $n=1000$ are marked with an asterisk. Some effects can be observed by tracing the asterisks in the different cloud plots. These asperities on the initial tool surface are not necessarily the highest asperities, but these are the fastest growing asperities. This effect can already be observed for the first ten product cycles as follows from the figures 5.9a and 5.9b. This effect is continued for the following product cycles according to the figures $5.10 \mathrm{a}$ and $5.10 \mathrm{~b}$. According to the figures, it seems that these asperities tend to develop a geometry with a width-height ratio of 5 , which is shown by the dashed lines in the figures 5.9 and 5.10. This effect cannot be observed for the galling calculations in general, it is related to this particular tribological system. Much smaller width-height ratios than 5 are unlikely, because in that case the asperities become mechanically too weak. Larger width-height ratios exist for situations when many asperities have a large value of $w_{\text {total }}$, for example, when the angle between the grinding direction of the tool surface and the sliding direction is large. One scratch belonging to such asperity (focussing on the asterisks only) is given in the cloud plots of 5.9 and 5.10 by the furthermost right asterisk.

An increasing height of asperities and a decreasing number of asperities that carry the normal force has consequences for the contact situation. If the lump growth is very local, then at that location, the surface is highly elevated over the neighbouring surface area. This will result in a normal force concentration. This force concentration leads to more penetration of the lumps in order to satisfy the normal force equilibrium. When the lumps are still small, only a small approach of the surfaces results in more asperity contacts, but this approach has to be increased for the case of higher, but fewer asperities. That situation makes the surface more sensitive to severe scratching of the lumps.

Most quantities that are given in table 5.1 are strongly dependent on the properties of contact bodies. On the other hand $p_{a}$ is strongly dependent on the process conditions and is a function of the location on the tool and the punch displacement in a deep drawing process. The effect of $p_{a}$ will be shown in the figures 5.11 and 5.12. In these figures the height and the number of asperities in contact, so the asperities still growing, are shown as a function of $p_{a}$ for $n=1000$. 


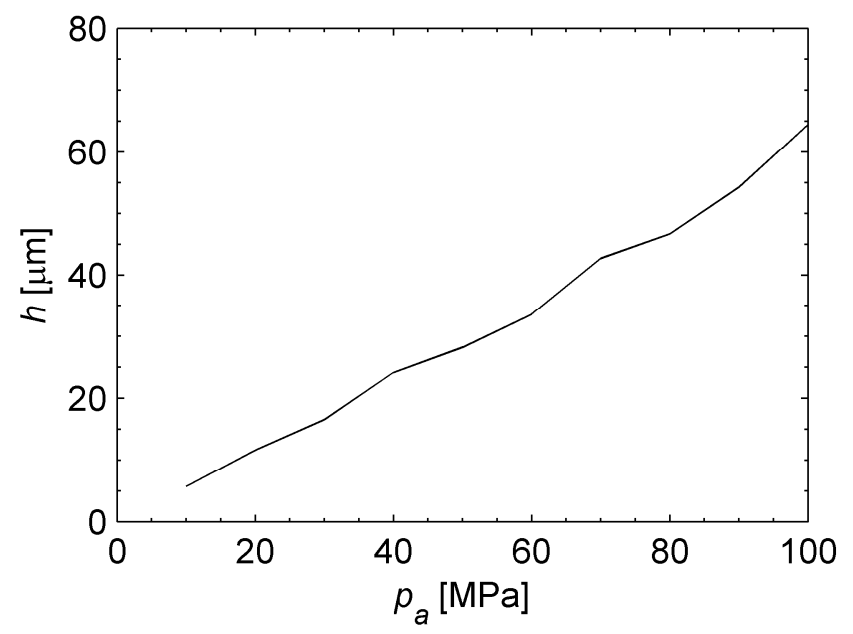

Figure 5.11. Height of the asperities as a function of $p_{a}$ for $n=1000$. The graph shows the mean height of the lumps that are still growing.

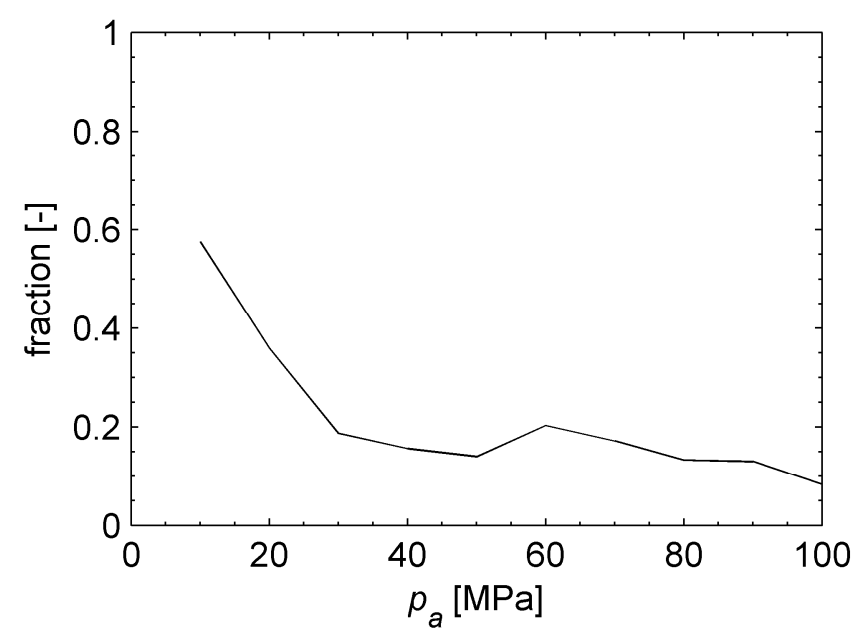

Figure 5.12. The fraction of the asperities that are still in contact as a function of $p_{a}$ for $n=1000$.

Figure 5.11 shows almost a linear relation between the apparent pressure $p_{a}$ and the galling effect, so proportionality between local contact pressure and galling sensitivity in a deep 
drawing process can be expected. For example on the die radius, a location with high contact pressure is a sensitive region for galling.

According to lump growth calculations with a single asperity as performed in section 4.5.7, the lump growth is very sensitive to the orientation of the asperity, as can be concluded from figure 4.36. The effect of the orientation is also investigated for the multi asperity case, using the measured (grinded) surface. In this investigation the orientation $\phi$ is defined as the angle between the grinding direction and the sliding direction. In the former calculations $\phi=10^{\circ}$. In figure 5.13 and 5.14 some results of the calculations are presented.

From these calculations it follows that the relation between the orientation and the lump growth is very weak for a multi asperity contact. This weak relation is in contrast to the single asperity case. In figure 5.13 the highest height is only 14 percent above the lowest. Analysing the calculation data in more detail, it reveals that the typical long asperities of a grinded surface behave in the manner such as presented in section 4.5.7. However, a grinded tool surface consists of long asperities, but also of short asperities, which means asperities with a major and minor radius that are almost equal. The lump growth of the short asperities is almost equal for all orientations, for the long asperities it is very sensitive for $\phi$. This effect explains in figure 5.14 the lower part of the graph for $\phi<30^{\circ}$. For the low values of $\phi$ the long asperities grow much less than the short ones, so after a number of cycles, more and more long asperities lose contact. The effect of $\phi$ on the growth in height, as shown in figure 5.13, is weaker. In figure 5.13 the situation is given for $n=1000$. For lower values of $n$ the effect of $\phi$ is stronger. For these lower values of $n$, when long asperities are still in contact, these asperities carry a part of the load, but for low values of $\phi$ they only contribute a little to the lump growth. When $n$ increases, the number of asperities that are in contact may reduce, but the growing speed is at that moment determined by the fast growing lumps that are still in contact. 


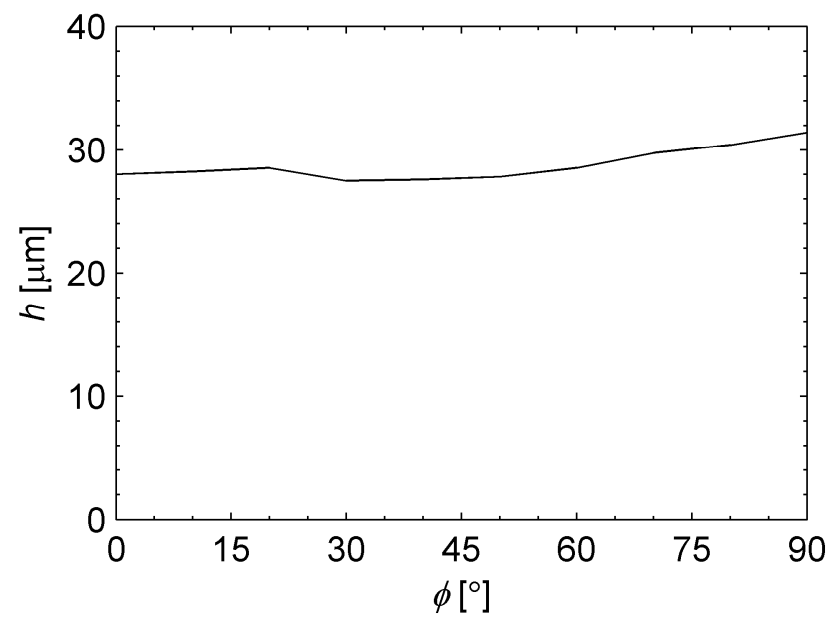

Figure 5.13. Lump growth of asperities as a function of $\phi$ for $n=1000$. The graph shows the mean height of the lumps that are still growing.

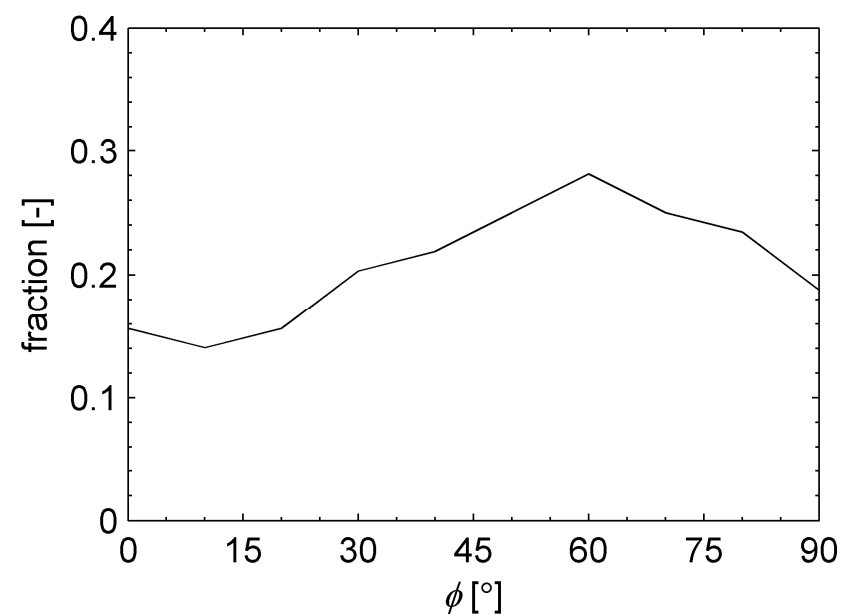

Figure 5.14. The fraction of the asperities that are still in contact as a function of $\phi$ for $n=1000$. 
To compare different materials, the strength of the transferred material has to be taken into account. This effect is shown in figures 5.15 and 5.16. The strength is given as the dimensionless shear strength $T$, defined as the shear strength divided by $p_{p l}$. When there is no hardening of the material during material transfer, the value of $T$ is 0.18 , which follows from equation (4.11), assuming that $p_{p l}$ equals the hardness of the sheet material. When there is hardening due to the transfer, the value of $T$ increases. So, the value of $T$ can be interpreted as the hardening effect on the transferred material.

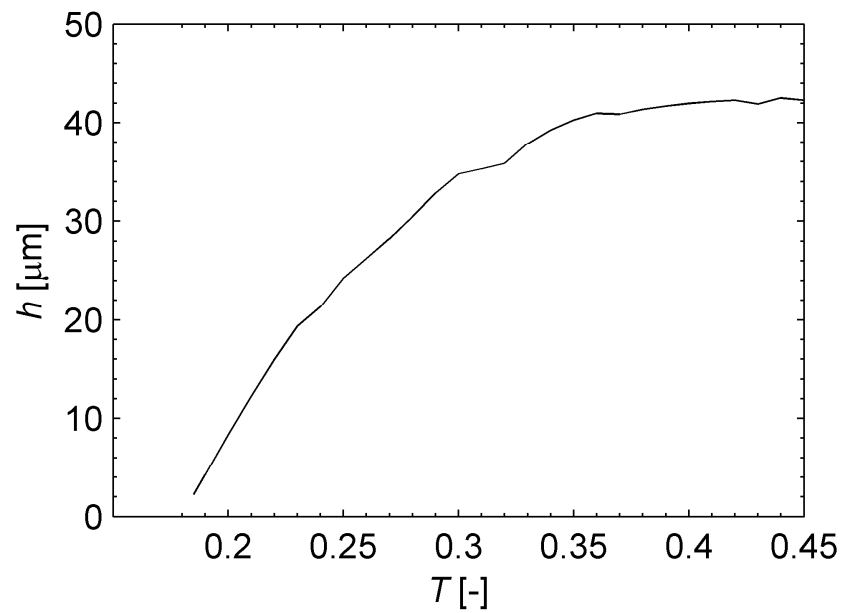

Figure 5.15. Height of the asperities as a function of $T$ for $n=1000$.

The graph shows the mean height of the lumps that are still growing. 


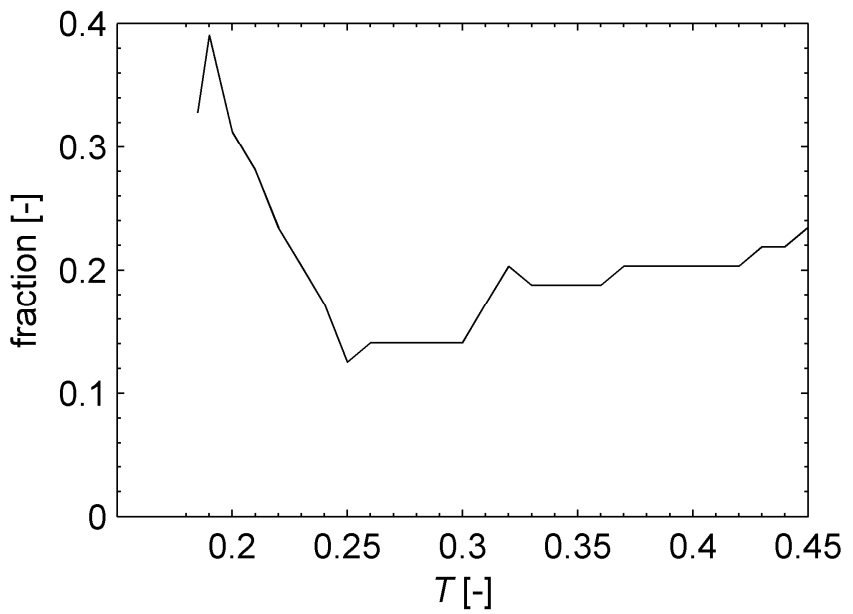

Figure 5.16. The fraction of the asperities that are still in contact as a function of $T$ for $n=1000$.

From figure 5.15 it follows that for an increasing value of $T$, lump growth increases. According to figure 5.16 the effect of $T$ on the fraction of asperities that is still in contact for $n=1000$ is significant for $T<0.25$. The fraction decreases rapidly for $0.19<T<0.25$. For increasing $T$, the lumps become stronger, so a set containing fewer lumps is able to resist the contact forces. When $T$ exceeds 0.25 , the strong relation with the fraction is no longer observed. The situation of $T=0.18$ is a special one, because material hardening is absent. When $T=0.18$ a lump cannot grow in height, but the tool surface can still be filled with transferred material between the asperities. Due to this effect the lowest asperities lose contact, which explains the part of the graph in figure 5.16 for $T<0.19$.

From these calculations it follows that when a sheet material has a stronger hardening effect due to material transfer, the lumps can grow more in height. So, less hardening results in fewer high lumps. This effect does not hold for almost no hardening. In that case it is calculated that a smeared layer is transferred to the tool surface, which is often visible when processing zinc coated steel.

The possibility of lump growth for work hardening materials can be observed in real practice on aluminium and stainless steel sheet material. Aluminium sheet AA5182 has a yield strength $\sigma_{y}$ of $130 \mathrm{MPa}$ and an ultimate strength $\sigma_{m}$ of $275 \mathrm{MPa}$. According to these values $T$ can get the value $(0.18 \times 275 / 130=) 0.38$. For $T=0.38$, the galling process operates in the region with maximum lump growth according to figure 5.15. Higher values of $T$ show no significant extra lump growth. Using the hardness of AA5182 sheet material, about $680 \mathrm{MPa}$ (see table 3.3), in equation (4.10) the yield strength is about $243 \mathrm{MPa}$. This 
value is much higher than the bulk yield strength, because the surface of sheet material is already hardened during production by the rolling process. This effect apparently lowers the value of $T$. However, another effect is that the ultimate strength is determined using the stress-strain curve that is based on the apparent stress, while in the case of galling the actual stress is relevant, which has a higher value than the apparent stress. Hence, it can be concluded that both terms in the ratio $T$, when applied to their surface, the yield strength as well as the surface ultimate strength, are underestimated when bulk properties are used in the calculation. Therefore, the ratio of bulk yield strength and bulk tensile strength will be used for calculating a good approximation of the $T$ value at the surface.

According to De Rooij [39], stainless steel AISI304 is characterized by $\sigma_{y}=195 \mathrm{MPa}$ and $\sigma_{m}=500-700 \mathrm{MPa}$. This results in $T \approx(0.18 \times 195 / 500 \approx) 0.55$. Similarly, steel A620 is characterized by $\sigma_{y}=270 \mathrm{MPa}$ and $\sigma_{m}=350 \mathrm{MPa}$. This gives $T=(0.18 \times 350 / 270=) 0.23$. These values correspond to the industrial experience that stainless steel is very sensitive to galling.

\subsubsection{Consequences for galling in deep drawing processes}

A galling model is developed for a multi asperity contact. Calculations have been performed for a model system. Two quantities that follow from the calculations are used as an indicator of the galling behaviour as mentioned in the block 'Galling Performance Indicator' in the scheme of figure 5.6. These quantities are the average lump height and the fraction of the asperities that are still in contact after a certain number of cycles. The difference between the lump height and the separation between the contacting surfaces can be used to determine the scratch depth caused by a lump. The lump height in combination with the fraction of asperities that are still in contact gives the sensitivity for scratching.

From the results that are obtained using the galling model some consequences can be given.

- A higher contact pressure results in more lump growth, because the fraction of the sheet material that comes in contact is larger due to this higher pressure. This means that locations like die radii are expected to be sensitive for galling.

- More work hardening of transferred material results in more lump growth. Due to the higher strength, a higher lump is still able to resist the contact forces. So, increasing the surface hardness of the sheet, for example by cold rolling, reduces lump growth in height and because of this the scratch depth.

- In the case of plastic bulk deformation in in-plane directions of the sheet the effective hardness of the sheet decreases, so $p_{p l}$ decreases. When the apparent contact pressure $p_{a}$ remains constant, $\alpha$ will increase, because a larger bearing area is needed in the case of a lower effective hardness. As a result $T$ increases, because the strength of the lump will now be normalized by a smaller value of $p_{p l}$. So, for the case of a lower effective hardness the lump growth increases by both of the 
effects that are mentioned in the two former points. This means that in a press, locations with high strains are sensitive to galling.

\subsection{Comparison between model and practice}

In order to validate the galling model developed in chapter 4 and the first sections of this chapter, tests are performed with the TNO slider-on-sheet tribometer [19], which is in principle the same tribometer as presented in [15]. The slider-on-sheet tribometer is a tester which is used to simulate the deep drawing contact on lab scale. The slider makes parallel tracks on sheet material, as shown in figure 5.17. The parallel tracks always ensure that a fresh part of sheet material is in contact with the slider. The slider is made of tool steel and represents the tool in the tests. Its shape is a disk with a radius in sliding direction and in perpendicular direction, in order to create a well defined contact. This slider is on every track in contact with fresh sheet material, like a real tool in every production cycle. The analogy of the TNO slider-on-sheet tribometer with the Ploughing Asperity Tester, as discussed in section 3.1, is that a sample, representing the tool, is always in contact with fresh sheet material. However, the TNO slider-on-sheet tribometer performs the tests on a larger scale. The slider corresponds to a part of the tool surface, instead of only one asperity. Further, the total sliding distance is in the order of real tools. The sliding tracks are made in $x$-direction. After a track, the slider is lifted from the sheet and returned to its starting $x$-position and moved $1 \mathrm{~mm}$ in $y$-direction after which the following track is made. In this way $1 \mathrm{~km}$ sliding distance is realized on one square metre of sheet material.

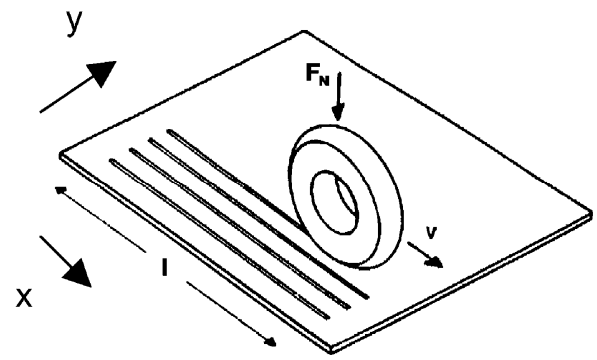

Figure 5.17. Schematic drawing of the TNO slider-on-sheet test, [19].

Two tests are performed that are compared with the developed galling model. In both tests the slider is made of cast iron GGG 70L, $R_{q}=1.0 \mu \mathrm{m}$. The diameter of the disk is $44 \mathrm{~mm}$, so the radius in sliding direction is $22 \mathrm{~mm}$, the radius in perpendicular direction is $6 \mathrm{~mm}$. In both tests the type of sheet material is also equal, namely aluminium AA 5182. The difference in the sheet material is the surface texture. One test is performed with a mill finished texture AA $5182 \mathrm{MF}, R_{q}=0.4 \mu \mathrm{m}$ and one with an electrical discharge texture 
AA $5182 \mathrm{EDT}, R_{q}=1.4 \mu \mathrm{m}$. The tests are performed with a normal load of $100 \mathrm{~N}$, the sliding velocity is $0.05 \mathrm{~m} \mathrm{~s}^{-1}$. The sheets are lubricated with $\mathrm{N} 6130$.

From an investigation of the plastic deformed tracks on the sheet and the operational conditions it follows that in the contact $p_{a} \approx 180 \mathrm{MPa}$. In combination with the hardness of the sheet material, $H=650 \mathrm{MPa}$, it follows that $\alpha=0.27$.

The tracks on the sheet of AA $5182 \mathrm{MF}$ are investigated after a total sliding length of the slider of $600 \mathrm{~m}$. The grooves that are formed within the tracks have a depth of about $5 \mu \mathrm{m}$. For AA 5182 EDT the tracks are investigated after a total sliding distance of the slider of $900 \mathrm{~m}$. The grooves that are formed in the track are in the order of the roughness and are hardly distinguishable from the roughness. Profiles of the tracks are shown in figures 5.18 and 5.19.

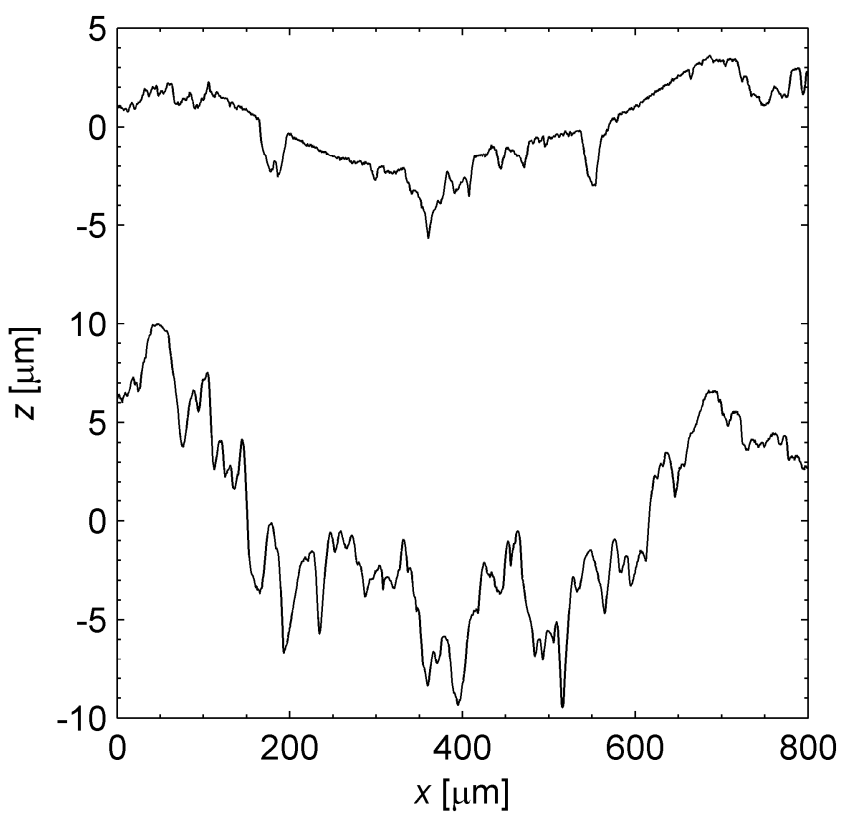

Figure 5.18. Height profiles on AA $5182 \mathrm{MF}$ sheet material of the first (top) and last (bottom) sliding track. 


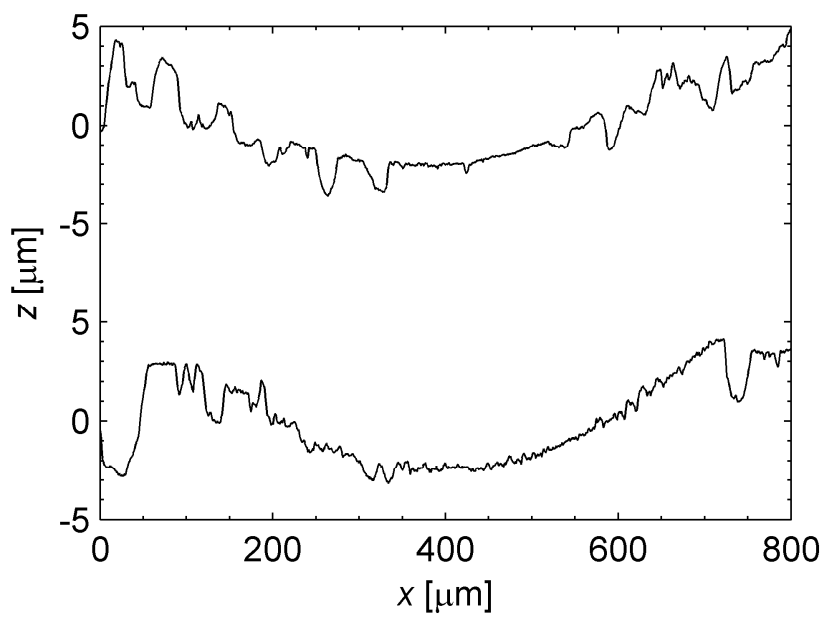

Figure 5.19. Height profiles on AA 5182 EDT sheet material of the first (top) and last (bottom) sliding track.

The contacts of the tests are also simulated in the galling model. Almost all parameters are equal for both tests, with the exception of the sheet surface texture. For the case of AA 5182 EDT the input of the model consists among other things of representative surface geometries, $\alpha=0.27$ and $\alpha_{\text {micro }}=0.5$, a strategy similar to the former section. The value of $\alpha_{\text {meso }}$ can now be determined from $\alpha$ and $\alpha_{\text {micro }}$. With these values, the contact on the meso scale can be determined followed by the contact on micro scale. For AA 5182 MF the meso scale has to be omitted, because the tool is much rougher than the sheet, so the assumption of the formation of plateaus on the sheet is no longer valid. Therefore, $\alpha_{\text {micro }}$ equals $\alpha$. So, the only important difference between the two calculations is the presence of the meso scale in the contact modelling. Other physical values that are used in the calculation are given in table 5.2. Some explanation will be given about the values in this table. The used value of $T$ for AA 5182 is derived in section 5.4. The value of $c_{f r}$ is adapted to scale the obtained results with the experiments. In the calculations of section 5.4 an arbitrary value of $c_{f r}$ is chosen for presenting the model. Here, the value can be adapted to the reality. Results of the calculations are shown in figure 5.20. 


\begin{tabular}{|l|l|l|}
\cline { 2 - 3 } \multicolumn{1}{c|}{} & Parameter & Value \\
\hline Material parameters & $T$ & 0.38 \\
\cline { 2 - 3 } & $H$ & $0.65 \mathrm{GPa}$ \\
\cline { 2 - 3 } & $\Delta \gamma$ & $1.1 \mathrm{~J} \mathrm{~m}^{-2}$ \\
\hline \multirow{5}{*}{ Other parameters } & $l_{\text {slide }}$ & $1 \mathrm{~m}$ \\
\cline { 2 - 3 } & $c_{f r}$ & $2 \cdot 10^{-8} \mathrm{~m}^{2} \mathrm{~J}^{-1}$ \\
\cline { 2 - 3 } & $\mu$ & 0.18 \\
\hline
\end{tabular}

Table 5.2. Physical parameters used in the galling model.

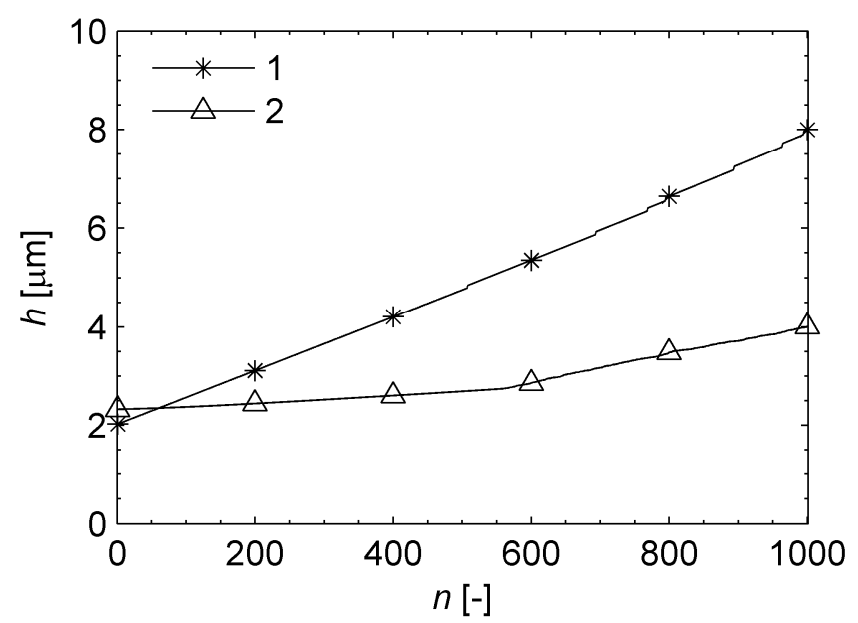

Figure 5.20. Height of the asperities as a function of the number of sliding tracks for AA $5182 \mathrm{MF}$ (graph 1) and AA 5182 EDT (graph 2).

The graphs show the mean height of the lumps that are still growing.

According to the calculations with the galling model the galling behaviour of AA 5182 sheet material is dependent on the surface texture. AA $5182 \mathrm{MF}$ is, according to the model, more galling sensitive than AA 5182 EDT. This is in agreement with the experiments on the TNO slider-on-sheet tribometer, see figures 5.18 and 5.19. The experiments performed with AA 5182 EDT show that there is almost no material transfer. According to the model, material transfer occurs, but the effect is small over the first 500 metres. After this distance the material transfer increases. 
The developed galling model is able to give the difference in galling trend in different situations. When compared with experiments, it does not give exactly the same results, but it already gives a distinction between different galling situations.

\subsection{Implementation of Galling Performance Indicator}

The calculated galling behaviour on micro and meso scale has to be translated to the macro scale in order to ascertain the galling behaviour on the different tool locations. Data obtained on the meso and micro scale, as presented in the former section of this chapter, has to be combined with data on the macro scale obtained by FEM simulations.

The galling data that is obtained on the meso and micro scale should be transformed to a form suitable for the combination with FEM data. Surface properties like adhesion properties, hardness and roughness are used on the meso and micro scale. These values cannot immediately be used on the macro scale. These factors should be captured in a galling impact factor $G$, as will be discussed later. When a homogeneous type of sheet material is used and the tools on all locations have the same surface properties, this factor $G$ is equal on every contact location. Factors that follow from operational conditions are the apparent contact pressure $p_{a}$ and the sliding distance $l_{\text {slide }}$. These factors are used on the smaller scales, but are obtained by FEM simulations. These factors will be used to derive the Galling Performance Indicator (GPI) on macro scale.

According to the galling calculations, that are performed in the former sections of this chapter, the lump growth is an (almost) linear function of $n$ and $p_{a}$, as shown in figures 5.7 and 5.11. As shown in figures 4.32 to 4.34, the lump growth is in fact an (almost) linear function of the total sliding length $n \cdot l_{\text {slide }}$, so the linearity that follows from figure 5.7 will now not be used for the number of products that are formed, but for $l_{\text {slide }}$. To investigate the galling effect for a deep drawing process, $l_{\text {slide }}$ is relevant for distinguishing different tool locations, in contrast to $n$, which is not a function of the tool location. Because $p_{a}$ varies during a production cycle, the GPI is formulated from these linear relations as an integral:

$$
I_{G P I}=G \int p_{a} d l
$$

Using equation (5.24) the GPI value $I_{G P I}$ is calculated for a location on the tool. The increment $d l$ is the incremental length of sheet material that slides along the tool. The integral has to be performed for one production cycle. Integrating $d l$ for one production cycle will result in the sliding length $l_{\text {slide }}$.

From a practical view of implementation, before using equation (5.24), a mapping of FEM data has to be performed on the tool. Usually, FEM software focuses on the product to be formed and so the physical quantities are only calculated for the blank. In section 2.2.1 it is mentioned that the modelled tools are used as geometrical constraints. Further, in the blank only the in-plane normal stresses and shear are calculated. The normal stress perpendicular 
on the sheet has to be obtained from the contact elements. These elements will also be used for mapping the data on the tools. A contact element consists of two sets of nodes. One set is connected to the sheet, the other set will be projected on the tool surface when it comes in the neighbourhood of the tool, see figure 5.21. When this projection results in a positive gap, the sheet and the tool are still not in contact, when it results in a negative gap, the contact elements determine the contact pressure as given in equation (2.1). The projection of these nodes gives the possibility of relating blank location to tool locations. The value of $p_{a}$ at a location of the tool can now be calculated by interpolation of the contact pressures at the nearest contact nodes that are projected on the tool. Further, by tracing the movement of the contact nodes over the tool surface, the increment $d l$ (for the discretized version of equation (5.24)) can be determined.

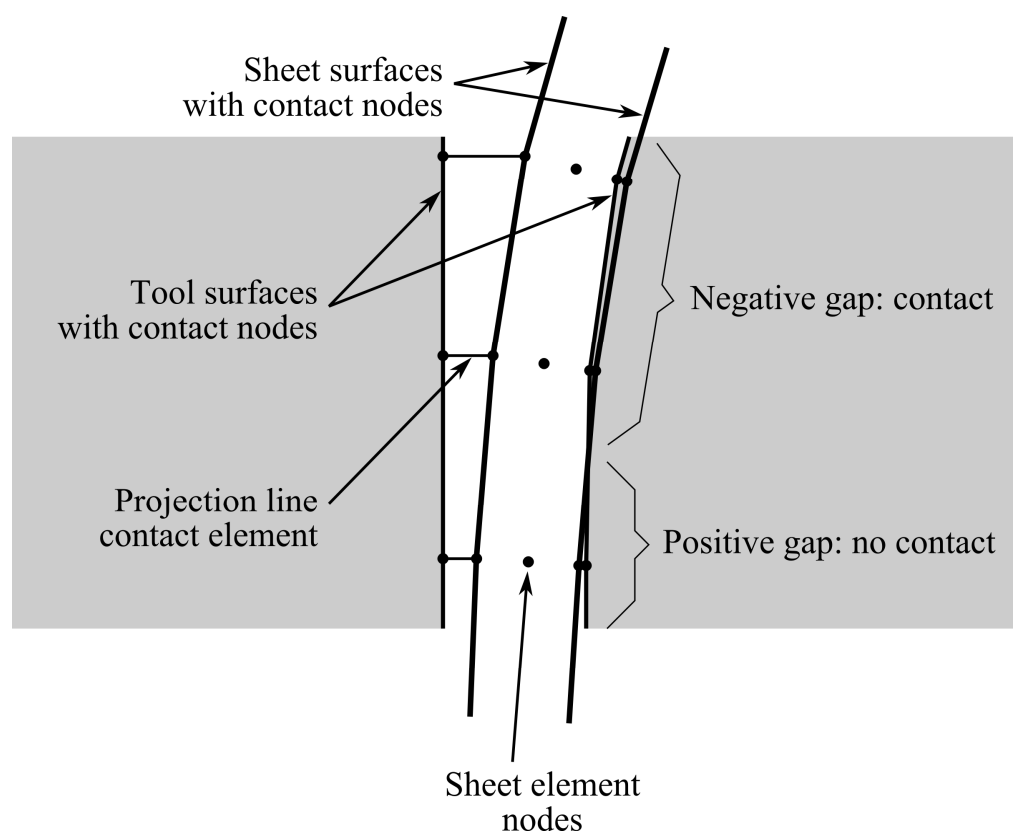

Figure 5.21. Part of sheet material and tools (grey areas) with sheet element nodes and contact elements. The tool at the left-hand side is not in contact, the tool at the right-hand side is partly in contact.

This strategy of calculating $I_{G P I}$ is applied to the FEM simulation of the deep drawing process of the cup as presented in figures 2.2 and 2.3 with contact penalty $C_{n}=400 \mathrm{~N} \mathrm{~mm}^{-3}$. In figure 5.22 results are shown of the calculations of $I_{G P I}$. In this figure, 
only the part of the integral of equation (5.24) is calculated; the value of $G$ is given a unit value.

In figure 5.22 only the effect is shown of the operational conditions $p_{a}$ and $l_{\text {slide }}$. This calculation can be performed over one production cycle. Because this result can be obtained in one cycle, the computational costs are relatively low. The value of $G$ has to be obtained by performing the calculations on the meso and micro scale. The value of $G$ equals the lump growth height divided by $p_{a}$ and $n \cdot l_{\text {slide }}$. By dividing the total lump growth by $n$, the galling effect is given for one production cycle. So, the increase in height $\Delta h$ due to galling can in general be expressed by:

$$
\Delta h=G p_{a} \Delta l
$$

For one product cycle in deep drawing $\Delta l=l_{\text {slide }}$. This is similar to wear, where the decrease in height is calculated with:

$$
\Delta h=k_{w} p_{a} \Delta l
$$

Although $G$ gives the galling effect for one production cycle, it cannot be obtained by calculating the galling effect after one production cycle on the meso and micro scale. As can be observed in figures 5.7 and 5.8 the first cycles show start-up effects. After a number of cycles the lump growth is more stable. To get a realistic value of $G$, the lump growth should be in the stage of stable lump growth. 


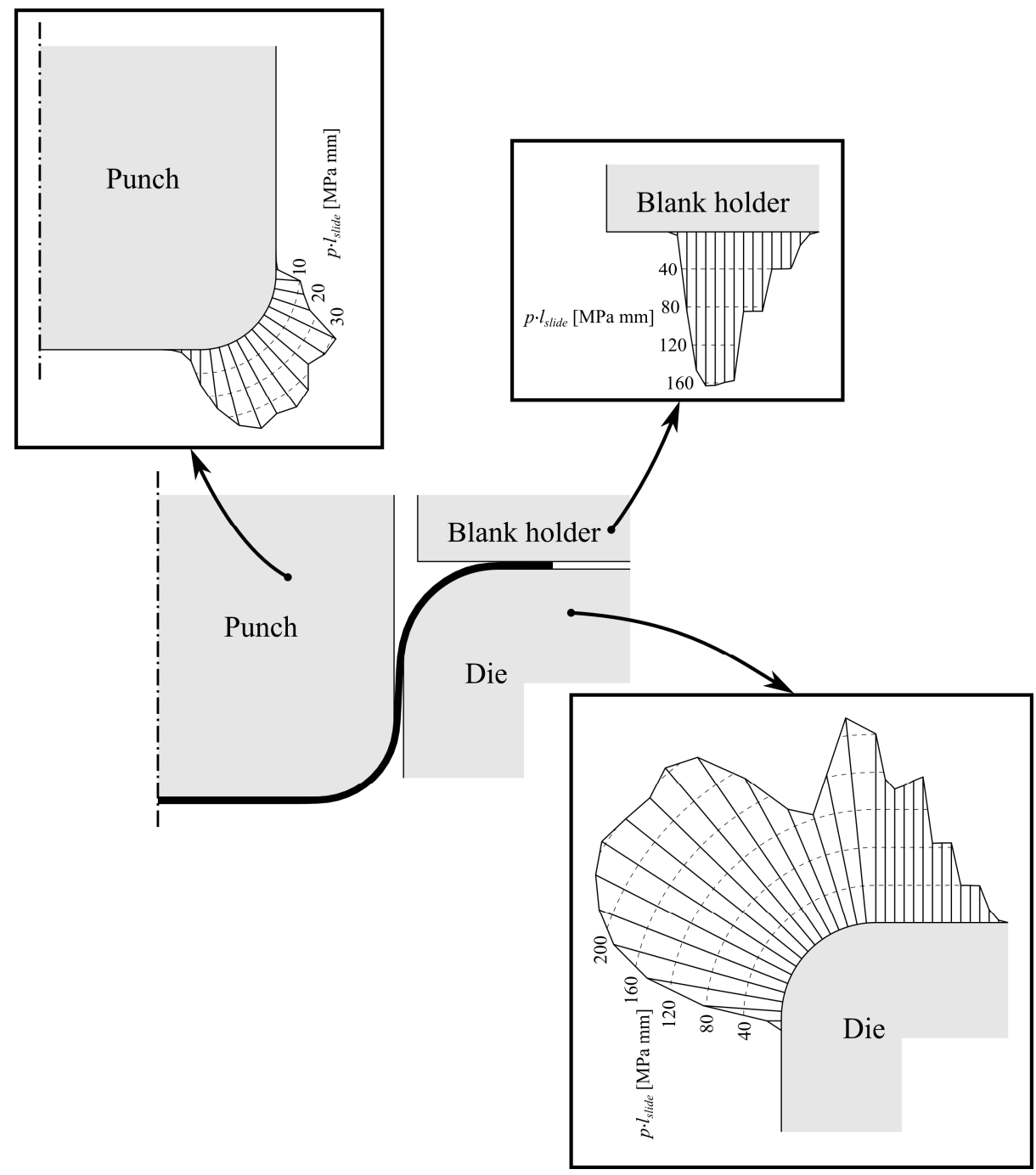

Figure 5.22. Deep drawing tools as assembly and individually with the values of $I_{G P I}$ plotted on it for $G=1$. The scale of $I_{G P I}$ graphs is different for the different tools.

In figure 5.22 the effect is shown of the operational condition on the galling behaviour. From these values it becomes clear that the punch is the least sensitive to galling. Although the highest value of $p_{a}$ is on the punch, the values of $l_{\text {slide }}$ are low, which results in low values of $I_{G P I}$. On the die and the blank holder, there is a combination of high values of $p_{a}$ and $l_{\text {slide, }}$, which results in high values of $I_{G P I}$. Further, on the die and blank holder the effect can be observed that the blank diameter reduces during forming. Due to the shorter contact 
time at the outer diameter of the die and the blank holder, the value of $I_{G P I}$ decreases in the radial direction.

The calculation that is presented in figure 5.22 is performed using equation (5.24). This equation is based on the galling model as presented in this chapter. For a galling model that takes more effects into account, for example the effective hardness due to strain of the sheet material and lubricant failure due to thermal effects, equation (5.24) has to be extended for these effects. That means that the integral is still a function of pressure and the sliding distance, but also of other quantities like strain and sliding velocity. In this way, the structure which is presented in this section can also be used for a more extensive galling model.

The procedure as presented before can be used to implement the GPI as a post processor for a FEM simulation of a deep drawing process. This post processor gives the galling effect on the different tool locations that come in contact with the blank. This post processor is a relatively low computational cost implementation. The integral of equation (5.24) has to be performed once for a deep drawing cycle. The value of $G$ has to be calculated beforehand on the meso and micro contact scale, which is a multi cycle calculation.

\subsection{Summary}

A galling model is developed for the multi asperity contact situation in a deep drawing process. From this galling model a Galling Performance Indicator (GPI) is formulated in such a way that it can be implemented as a post processor for FEM simulations. In order to make the transition from the single asperity galling model as presented in chapter 4 to the multi asperity model and the GPI the following steps are taken.

- A contact model is developed that can deal with a meso and micro contact scale. On the micro scale polyhedron shaped asperities with a hexagon base are formulated that can be used on asperity level in the single asperity model.

- A multi asperity galling model is developed. In this model the single asperity model is applied to all the contacts that are determined using the contact model.

- Calculations are performed with the multi asperity galling model. From these calculations it follows that the galling effect is (almost) proportional to the contact pressure and to the sliding distance. Further, the galling behaviour is dependent on the dimensionless strength $T$ of the lumps that are formed on the asperities. Although the lump growth is rather sensitive to the orientation on the single asperity scale, the orientation effect on a grinded surface is hardly noticeable. The spread of the orientations over the whole set of asperities almost cancelled out the orientation effect.

- TNO slider-on-plot tests are performed to validate the galling model. According to these tests the galling model is in reasonable agreement with reality. 
- A GPI is formulated on the macro scale. This indicator can be implemented as a post processor for deep drawing FEM simulations. This GPI is composed of a galling impact factor $G$ and the operational conditions $p_{a}$ and $l_{\text {slide }}$. $G$ can be determined with the developed galling model on meso and micro scale, the operational conditions are obtained by FEM simulation. 


\section{Conclusions and recommendations}

In this thesis a model is presented that is developed for predicting the galling behaviour in deep drawing processes. In this chapter the conclusions of the research and recommendations for further research are given. The main conclusions obtained from this research are given below.

\subsection{Conclusions}

\section{Chapter 3}

In order to investigate the galling behaviour on micro scale, single asperity experiments are performed with a tip of tool steel that represents a tool asperity which comes in contact with sheet material. From these single asperity tests it follows that material is transferred to the tool.

For unlubricated contacts a lump grows in height and in front of the asperity. Due to this growth a new attack angle is formed in the contact. The new attack angle is material dependent.

A trend can be observed that for longer sliding distances, more material transfers from the sheet to the tool.

\section{Chapter 4}

A single asperity lump growth model is developed. The model considers galling as a process that is based on two stages. In the first stage, material is transferred from the sheet to the tool, which forms a transfer layer on the asperity. In the second stage, a part of the transferred layer shears off, when the asperity with lump is not strong enough to resist the contact forces. When the mechanical stresses exceed the material strength in the lump, a part of the lump shears from it, in order to get a mechanically stable stress state within the lump. In this way, the model is able to explain the formation of a new attack angle, dependent on the strength of the lump.

The adhesion and sliding distance are important factors of the lump growth. 


\section{Chapter 5}

From calculations with the multi asperity galling model, it follows that the galling effect is proportional to the contact pressure and to the sliding distance. Further, the galling behaviour is dependent on the dimensionless strength $T$ of the lumps that are formed on the asperities.

Good agreement is found between model and galling tests.

The developed galling model is implemented as a post processor for finite element simulations and applied to the drawing of a cup. The areas, to be known as 'galling areas', are predicted well by the Galling Performance Indicator $I_{G P I}$.

\subsection{Discussion}

According to the research that is presented in this thesis, the impact of a number of parameters on the galling behaviour becomes clear. Important parameters are the adhesion $\Delta \gamma$, the total sliding length $l_{\text {slide }} \cdot n$, the contact pressure $p_{a}$ and the strength of the lumps $T$. The sliding length is in most situations a value that is already determined by the product design and so, this parameter cannot be varied. The other parameters have more space for variation. Usually, the adhesion is reduced by lubrication. Further, by changing the die radii the contact pressure can be changed. The strength is mainly determined by the selected material, but the value of $T$ is also influenced by strain. In order to get more insight in these issues a number of recommendations for further research are given in the following section.

\subsection{Recommendations}

In the chapters 4 and 5 a galling model is formulated. To reduce galling, systems are lubricated. According to the model of Van der Heide [20], as mentioned in chapter 2, galling does not take place if the local temperatures (at asperity level) do not exceed a critical temperature, $\Theta<\Theta_{c r}$. So the implementation of thermal effects in the galling model results in a useful extension for the Galling Performance Indicator $I_{G P I}$. In the Galling Performance Indicator the galling factor $G$ becomes:

$$
\begin{array}{ll}
G=0 & \text { if } \Theta<\Theta_{c r} \\
G \neq 0 & \text { if } \Theta \geq \Theta_{c r}
\end{array}
$$

The strain of the sheet material influences the value of $T$ of the lumps, because of its influence on the effective hardness. The model presented in this thesis is focussed on the hardness of the material in the case of no bulk deformation. Deep drawing is a forming 
process, so the effect of the strain is present in reality. Further, locations with high values of $I_{G P I}$ can also be locations with high strains. It is therefore recommended that the effect of strain on $T$ is taken into account in the Galling Performance Indicator.

The model is based on a steady state contact pressure. In a deep drawing process the pressure varies at a certain tool location as a function of the punch position. The number of asperities in those contacts will change as a function of the punch displacement. A consequence of this effect is that the highest asperities can grow all the time, while other less high asperities can grow only at high contact pressures. So, a possible influence of large pressure fluctuations can be that the highest asperities grow more than the other asperities in height and that these asperities can scratch deeper into the sheet material at the moment of high contact pressure. It is recommended to investigate this effect on the meso and micro scale. 


\section{Nawoord}

Dit onderzoek is uitgevoerd als een project van het M2i. Het M2i wil ik bedanken voor de mogelijkheid die geboden is om dit onderzoek uit te kunnen voeren.

Het onderzoek is uitgevoerd aan de Universiteit Twente binnen de leerstoel Oppervlaktetechnologie en Tribologie. Binnen deze groep ben ik afgestudeerd voor de opleiding Werktuigbouwkunde. Aansluitend werd de mogelijkheid geboden om het promotieonderzoek te verrichten waarover dit proefschrift handelt.

De leden van de leerstoel Oppervlaktechnologie en Tribologie wil ik bedanken voor de samenwerking, ondersteuning en de geboden gezelligheid. Matthijn de Rooij en Dik Schipper, respectievelijk de assistent promotor en promotor, wil ik in het bijzonder bedanken. Matthijn wil ik bedanken als dagelijkse begeleider van het onderzoek. Hij was daarmee het eerste aanspreekpunt voor wat betreft het onderzoek. Zijn kamer stond altijd open voor vragen en het discussiëren over problemen binnen het onderzoek en waar nodig stuurde hij het onderzoek bij. Met Dik waren de contacten minder intensief, maar ook hij stond altijd klaar voor het meedenken over problemen. Verder wil ik Willie Kerver, Walter Lette en Erik de Vries bedanken voor de technische ondersteuning bij het uitvoeren van de experimenten en Belinda Bruinink voor haar bijdrage als secretaresse. Van de promovendi wil ik in het bijzonder Jan Willem Sloetjes, Radu Popovici, Ellen van den Bos, Dinesh Karupannasamy, Agnieszka Winogrodzka en Adeel Yaqoob bedanken waarmee ik de afgelopen jaren op een prettige manier de werkkamer heb gedeeld.

Daarnaast wil ik mijn vrouw Hendrikje bedanken voor de steun en liefde die ik van haar gekregen heb. Boven alles komt de dank aan de Heere toe die de kracht en het verstand gaf om dit werk te kunnen doen.

Gerrit van der Linde Staphorst, oktober 2011 



\section{Appendix A: Stresses in pyramidal and hexagon based asperities}

\section{A.1 Introduction}

A statics analysis will be given of a pyramid shaped asperity, as given in chapter 4 . In the last section an extension is made for the hexagon based asperity. The characteristics and assumptions of the pyramid shaped asperity model are presented in section 4.3.2. The geometry of the tip is shown in figure A.1. The normal and tangential stress components on the face $A B C \sigma_{A B C}$ and $\tau_{A B C}$ will be calculated. The results of these values will be used for further analysis.

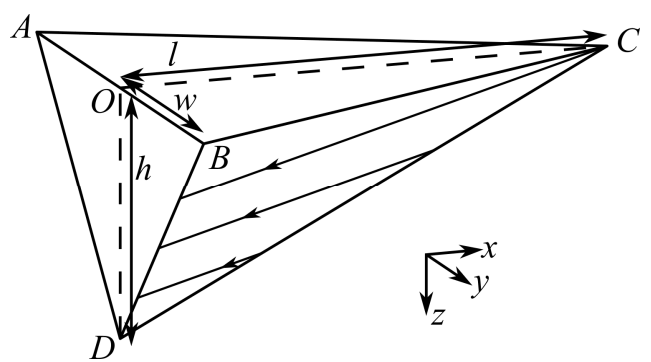

Figure A.1.Tip geometry with its dimension and flow lines on BDC of plastic deforming material.

Given are:

- the dimensions: $l, w$ and $h$.

- the plastic normal stress $p_{p l}$ on face $B D C$, pointing inward the asperity.

- the plastic tangential stress $\tau_{p l}$ in the direction of $\vec{t}$. The absolute value of $\tau_{p l}$ is given by $\tau_{p l}=\mu p_{p l}$. The vector $\vec{t}$ is the tangent vector of BDC as close as possible to the direction of the plastic flow in the far field. This tangent is illustrated in figure A. 1 by the lines with arrows on face $B D C$ and is given by:

$$
\vec{t}=\sqrt{\frac{w^{2}+h^{2}}{(w h)^{2}+(h l)^{2}+(l w)^{2}}}\left(-l, h \frac{w h}{w^{2}+h^{2}}, w \frac{w h}{w^{2}+h^{2}}\right)
$$

- an assumed constant tangential stress $\tau_{A B C}$ and a linear function for the value normal stress $\sigma_{A B C}$ that is a function of the $x$-coordinate.

- the assumption that the tangential stress $\tau_{A B C}$ is constant and the normal stress $\sigma_{A B C}$ is a function of the $x$-coordinate according to: 


$$
\sigma_{A B C}=-C\left(\delta_{0}+\theta x\right)
$$

containing a spring stiffness $C$, deformation $\delta_{0}$ at $x=0$ and an angle $\theta$. These are virtual stiffness and deformations to get a description of $\sigma_{A B C}$ and have no physical meaning.

The stresses on the face $A B C$ that are calculated are defined as follows:

- Normal stress $\sigma_{A B C}$ acts normal to $A B C$ and a positive value points outward the asperity.

- The tangential stress $\tau_{A B C}$ acts tangent to $A B C$ in the direction of the positive $x$-axis.

In the calculations, some lengths and surface areas are used multiple times. These are defined as follows:

- $\quad$ area of the triangle $B D C$ :

$$
A_{B D C}=\frac{1}{2}|B D \times B C|=\frac{1}{2} \sqrt{(w h)^{2}+(h l)^{2}+(l w)^{2}}
$$

- normal vector of $B D C$ :

$$
\vec{n}_{B D C}=\frac{(w h, h l, l w)}{\sqrt{(w h)^{2}+(h l)^{2}+(l w)^{2}}}
$$

- $\quad$ area of the triangle $A B C$ :

$$
A_{A B C}=w l
$$

\section{A.2 Equilibrium calculations}

From equilibrium in the $x$ and $z$-directions the stresses $\sigma_{A B C}$ and $\tau_{A B C}$ are calculated. In these calculations, the focus is on the $x z$-plane. The $y$-direction is of importance to determine the direction vectors of the plastic stresses as given in equations (A.1) and (A.4). From these vectors the stress components in the $x$ and $z$-directions can be derived. In further analyses, the $y$-components cancel out, because of the symmetry in the $x z$-plane. In the following sections the equilibrium is calculated in $x$ and $z$-direction and the moment in the $x z$-plane. From these equilibrium calculations follow $\sigma_{A B C}$ and $\tau_{A B C}$.

\section{A.2.1 Equilibrium in $x$-direction}

The equation of equilibrium in the $x$-direction results in the following equations:

$$
\begin{gathered}
\Sigma F_{x}=0 \\
-\int p_{p l} \vec{n} \cdot \vec{x} d A+\int \tau_{p l} \vec{t} \cdot \vec{x} d A+\int \tau_{A B C} d A=0
\end{gathered}
$$




$$
-p_{p l} w h-\tau_{p l} l \sqrt{w^{2}+h^{2}}+\tau_{A B C} A_{A B C}=0
$$

From these equations follows the value of $\tau_{A B C}$ :

$$
\tau_{A B C}=\frac{1}{A_{A B C}}\left(p_{p l} w h+\tau_{p l} l \sqrt{w^{2}+h^{2}}\right)=\frac{p_{p l}}{A_{A B C}}\left(w h+\mu l \sqrt{w^{2}+h^{2}}\right)
$$

\section{A.2.2 Equilibrium in z-direction}

The equations of vertical equilibrium are given in the relations below:

$$
\begin{gathered}
\Sigma F_{z}=0 \\
-\int p_{p l} \vec{n} \cdot \vec{z} d A+\int \tau_{p l} \vec{t} \cdot \vec{z} d A+\int \sigma_{A B C}(x) \vec{n} \cdot \vec{z} d A=0 \\
-p_{p l} w l+\tau_{p l} \frac{w^{2} h}{\sqrt{w^{2}+h^{2}}}+C \delta_{c} A_{A B C}=0
\end{gathered}
$$

In the last term of the right-hand side of equation (A.12) the symbol $\delta_{c}$ gives the value of the virtual displacement $\delta$ at location $x=x_{c}$, where $x_{c}$ is the $x$-coordinate of the centroid of face $A B C$. From equilibrium in the $z$-direction $\sigma_{A B C}$ cannot be determined over the whole face $A B C$, but using (A.2) it can be derived that $\delta_{c}$ is proportional to the mean value of $\sigma_{A B C}$. This gives the following:

$$
\delta_{c}=\frac{1}{C A_{A B C}}\left(p_{p l} w l-\tau_{p l} \frac{w^{2} h}{\sqrt{w^{2}+h^{2}}}\right)=\frac{p_{p l}}{C A_{A B C}}\left(w l-\mu \frac{w^{2} h}{\sqrt{w^{2}+h^{2}}}\right)
$$

\section{A.2.3 Moment equilibrium}

The equation of moment equilibrium around to the $y$-axis is as given in the relations below:

$$
\begin{gathered}
M_{y y}=\left(\int \vec{r} \times d \vec{F}\right) \cdot \vec{y}=\int z d F_{x}-\int x d F_{z}=0 \\
-\int z p_{p l} \vec{n} \cdot \vec{x} d A+\int x p_{p l} \vec{n} \cdot \vec{z} d A+\int z \tau_{p l} \vec{t} \cdot \vec{x} d A-\int x \tau_{p l} \vec{t} \cdot \vec{z} d A-\int x \sigma_{A B C} \vec{n} \cdot \vec{z} d A=0 \\
\frac{1}{3} p_{p l} w\left(l^{2}-h^{2}\right)-\frac{\tau_{p l} l}{3}\left(h \sqrt{w^{2}+h^{2}}+\frac{w^{2} h}{\sqrt{w^{2}+h^{2}}}\right)-C\left(\delta_{c} x_{c} A_{A B C}+I_{c y y} \theta\right)=0
\end{gathered}
$$

In (A.14) $\vec{r}$ and $\vec{F}$ are respectively the location and the force vector. In the last equation $I_{c y y}$ means the area moment of inertia of face $A B C$ with respect to the line $x=x_{c}$. From vertical and moment equilibrium, equations (A.13) and (A.16), it follows: 


$$
\theta=\frac{p_{p l}}{3 C I_{c y y}}\left(w\left(l^{2}-h^{2}-3 x_{c} l\right)+\frac{\mu h}{\sqrt{w^{2}+h^{2}}}\left(3 x_{c} w^{2}-l\left(2 w^{2}+h^{2}\right)\right)\right)
$$

The normal stress $\sigma_{A B C}$ can be found using (A.2), (A.13) and (A.17). The definition of $A_{A B C}$, $x_{c}$ and $I_{c y y}$ is worked out in section A.4.

\section{A.3 Results}

In A.2 relations are presented to calculate $\sigma_{A B C}$ and $\tau_{A B C}$. To present these results, dimensionless relations are used. The lengths are normalized by $l$, that results in a dimensionless width $\bar{w}$, height $\bar{h}, x$-coordinate $\bar{x}$, centroid $\bar{x}_{c}$, area $\bar{A}_{A B C}$ and area moment of inertia $\bar{I}_{c y y}$.

$$
\begin{gathered}
\frac{\tau_{A B C}}{p_{p l}}=\frac{1}{\bar{A}_{A B C}}\left(\bar{w} \bar{h}+\mu \sqrt{\bar{w}^{2}+\bar{h}^{2}}\right) \\
\frac{\sigma_{A B C}}{p_{p l}}=-\frac{\bar{w}}{\bar{A}_{A B C}}\left(1-\frac{\mu \bar{w} \bar{h}}{\sqrt{\bar{w}^{2}+\bar{h}^{2}}}\right)+\frac{1}{3 \bar{I}_{c y y}}\left(\bar{w}\left(\bar{h}^{2}-1+3 \bar{x}_{c}\right)+\frac{\mu \bar{h}\left(2 \bar{w}^{2}+\bar{h}^{2}-3 \bar{x}_{c} \bar{w}^{2}\right)}{\sqrt{\bar{w}^{2}+\bar{h}^{2}}}\right)\left(\bar{x}-\bar{x}_{c}\right)
\end{gathered}
$$

\section{A.4 Geometrical properties of face $A B C$}

In equations (A.18) and (A.19) (equal to equations (4.8) and (4.9)) some properties of the surface $A B C$ are referred to their general form, namely centroid $\bar{x}_{c}$, area $\bar{A}_{A B C}$ and area moment of inertia $\bar{I}_{c y y}$. This general formulation makes the equations more flexible as is shown for example in sections 4.3.4 to 4.3.7.

The properties are defined as follows (in their dimensional form):

$$
\begin{gathered}
A_{A B C}=\int d A \\
x_{c}=\frac{1}{A_{A B C}} \int x d A \\
I_{c y y}=\int\left(x-x_{c}\right)^{2} d A=\int x^{2} d A-x_{c}{ }^{2} A_{A B C}=I_{y y}-x_{c}{ }^{2} A_{A B C}
\end{gathered}
$$

In (A.22) $I_{y y}$ is defined as:

$$
I_{y y}=\int x^{2} d A
$$

The dimensions of face $A B C$ are shown in figure A.2. The face is here presented in its 'flexible' form as used in section 4.3.4 and 4.3.6 and in (the explanation of) equation (4.16), 
that means, with extension of triangle $A E B$ and crack at point $C$. In the initial model formulation in section 4.3.2 $l_{b}$ and $l_{c r}$ are zero. In section 4.3.4 $l_{b}$ is introduced and in section 4.3.6 $l_{c r}$.

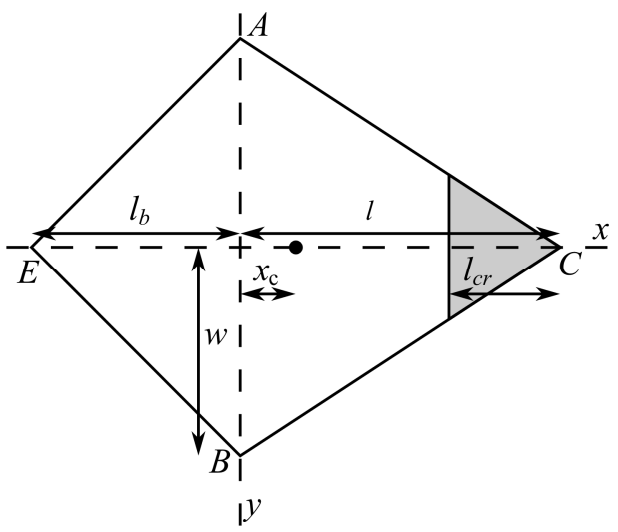

Figure A.2. Dimensions of face ABC, including the adaptations of the extension with triangle $A E B$ and the formation of a crack at point $C$.

Given the dimensions as shown in figure A.2, equations (A.20), (A.21) and (A.23) become:

$$
\begin{gathered}
A_{A B C}=w\left(l\left(1-\frac{l_{c r}}{l}\right)\left(1+\frac{l_{c r}}{l}\right)+l_{b}\right) \\
x_{c}=\frac{w}{3 A_{A B C}}\left(l^{2}\left(1-\frac{l_{c r}}{l}\right)^{2}\left(1+2 \frac{l_{c r}}{l}\right)-l_{b}^{2}\right) \\
I_{y y}=\frac{w}{6}\left(l^{3}\left(1-\frac{l_{c r}}{l}\right)^{3}\left(1+3 \frac{l_{c r}}{l}\right)+l_{b}^{3}\right)
\end{gathered}
$$

Using (A.24) to (A.26) in (A.22) gives $I_{c y y}$. The dimensionless form of $A_{A B C}, x_{c}$ and $I_{c y y}$ is obtained by dividing the quantities by respectively $l^{2}, l$ and $l^{4}$.

\section{A.5 Geometrical variation of geometry}

In section 4.5 an asperity geometry is used in the galling model that is an extension of the geometry as presented in the previous section of this appendix. The results that are generated before can be reused with some adaptations. These adaptations will be given below. 
In section 4.5 an asperity is proposed with a hexagon base as is shown in figure 4.23. This base is divided into three sections, where I and III have pyramidal shape and section II a prismatic shape.

To calculate the forces, equation (A.7) will be used. In equation (A.7) a force equilibrium equation is given. Here, only the first two integrals that calculate the plastic forces will be used. In the current situation the integrals contain constant values. So the integral can be reduced to a multiplication of the plastic stress, the inner product result and the area. The plastic stress is taken as constant, the inner product and the area has to be calculated. The inner product can be calculated as the normal and the tangent unit vectors are known. These can be calculated using equations (A.1) and (A.4) in an adapted form. In equations (A.1) and (A.4) $l, w$ and $h$ are used to give the orientation of the plane. For the current situation $w$ has to be replaced by the following to give the orientation:

- Section I: $w \rightarrow \frac{w_{\mathrm{I}} l}{l+w_{\mathrm{I}} \cos \alpha}$

- Section II: $w \rightarrow l \cot \alpha$

- Section III: $w \rightarrow \frac{w_{\mathrm{III}} l}{l-w_{\mathrm{III}} \cos \alpha}$

For section I and II this can be derived using the figures A.3 and A.4. These figures give the orientation and the dimensions of the contact planes. Using these figures the contact area can also be found.

For section I the forces will be derived using figure A.3. The area and the normal and tangent unit vectors of face $A G F$ are given by:

$$
\begin{gathered}
A_{A G F}=\frac{1}{2}|G F \times G A|=\frac{1}{2} \sqrt{\left(w_{\mathrm{I}} h\right)^{2}+h^{2}\left(w_{\mathrm{I}} \tan \alpha+l\right)^{2}+\left(l w_{\mathrm{I}}\right)^{2}} \\
\vec{n}_{A G F}=\frac{\left(w_{\mathrm{I}} h, h\left(l+w_{\mathrm{I}} \tan \alpha\right), l w_{\mathrm{I}}\right)}{2 A_{A G F}} \\
\vec{t}=\frac{\sqrt{\left(w_{\mathrm{I}} l\right)^{2}+h^{2}\left(l+w_{\mathrm{I}} \tan \alpha\right)^{2}}}{2 A_{A G F}}\left(-1, \frac{w_{\mathrm{I}} h^{2}\left(l+w_{\mathrm{I}} \tan \alpha\right)}{\left(2 A_{A G F}\right)^{2}-\left(w_{\mathrm{I}} h\right)^{2}}, \frac{l w_{\mathrm{I}}{ }^{2} h}{\left(2 A_{A G F}\right)^{2}-\left(w_{\mathrm{I}} h\right)^{2}}\right)
\end{gathered}
$$

Using equations (A.27a) to (A.27c) the forces in the $x$ and $y$-direction can be calculated using the first two integrals of equation (A.7). For the $y$-direction the unit $x$-vector has to be replaced by the unit $y$-vector in the integrals. This gives the following forces:

$$
\begin{gathered}
F_{A G F_{-} x}=-\frac{1}{2} p_{p l} w_{\mathrm{I}} h-\frac{1}{2} \tau_{p l} \sqrt{\left(w_{\mathrm{I}} l\right)^{2}+h^{2}\left(l+w_{\mathrm{I}} \tan \alpha\right)^{2}} \\
F_{A F_{-} y}=-\frac{1}{2} p_{p l}\left(l+w_{\mathrm{I}} \tan \alpha\right) h+\frac{1}{2} \tau_{p l} \frac{w_{\mathrm{I}} h^{2}\left(l+w_{\mathrm{I}} \tan \alpha\right)}{\sqrt{\left(l w_{\mathrm{I}}\right)^{2}+h^{2}\left(l+w_{\mathrm{I}} \tan \alpha\right)^{2}}}
\end{gathered}
$$




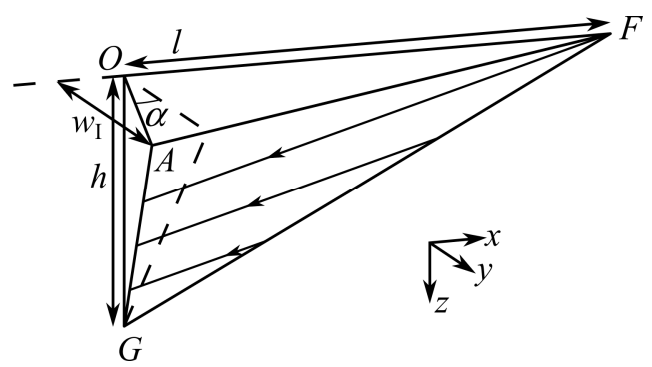

Figure A.3. Frontal part of section I of the asperity.

For section II the forces will be derived using figure A.4. The area and the normal and tangent unit vectors of face $A G F$ are given by:

$$
\begin{gathered}
A_{E F G H}=|F G \times F E|=w_{\mathrm{II}} \sqrt{h^{2}\left(1+\tan ^{2} \alpha\right)+l^{2}} \\
\vec{n}_{E F G H}=\frac{(h, h \tan \alpha, l)}{\sqrt{h^{2}+h^{2} \tan ^{2} \alpha+l^{2}}} \\
\vec{t}_{E F G H}=\sqrt{\frac{l^{2}+h^{2} \tan ^{2} \alpha}{h^{2}(1+\tan \alpha)^{2}+l^{2}}}\left(-1, \frac{h^{2} \tan \alpha}{l^{2}+h^{2} \tan ^{2} \alpha}, \frac{l h}{l^{2}+h^{2} \tan ^{2} \alpha}\right)
\end{gathered}
$$

Using equations (A.29a) to (A.29c) the following forces in the $x$ and $y$-direction are found:

$$
\begin{gathered}
F_{E F G H_{-} x}=-p_{p l} w_{\mathrm{II}} h-\tau_{p l} w_{\mathrm{II}} \sqrt{l^{2}+h^{2} \tan ^{2} \alpha} \\
F_{E F G_{-} y}=-p_{p l} w_{\mathrm{II}} h \tan \alpha+\tau_{p l} w_{\mathrm{II}} \frac{h^{2} \tan \alpha}{\sqrt{l^{2}+h^{2} \tan ^{2} \alpha}}
\end{gathered}
$$

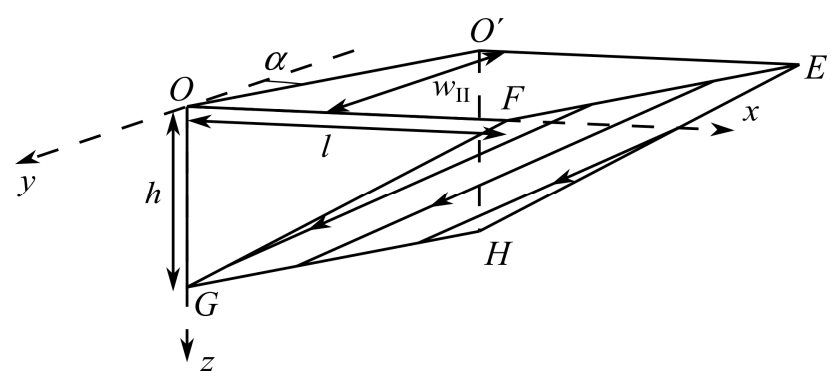

Figure A.4. Frontal part of section II, an oblique prism, of the asperity. 
Section III is more or less a mirrored version of section I. The equations (A.28a) and (A.28b) can be used again with small adaptations. In both equations the variable $\alpha$ should be replaced with $-\alpha$, equation (A.28b) should be multiplied by -1 and $w_{\mathrm{I}}$ replaced by $w_{\text {III }}$. Summation of the forces as derived above for the three section results in the total forces in $x$ and $y$-direction:

$$
\begin{gathered}
F_{x}=-p_{p l} h\left(\frac{1}{2} w_{\mathrm{I}}+w_{\mathrm{II}}+\frac{1}{2} w_{\mathrm{III}}\right) \\
-\tau_{p l}\left(\frac{1}{2} \sqrt{\left(w_{\mathrm{I}} l\right)^{2}+h^{2}\left(l+w_{\mathrm{I}} \tan \alpha\right)^{2}}\right. \\
+w_{\mathrm{II}} \sqrt{l^{2}+h^{2} \tan ^{2} \alpha} \\
\left.+\frac{1}{2} \sqrt{\left(w_{\mathrm{III}} l\right)^{2}+h^{2}\left(l-w_{\mathrm{III}} \tan \alpha\right)^{2}}\right) \\
F_{y}=-p_{p l} h \tan \alpha\left(\frac{1}{2} w_{\mathrm{I}}+w_{\mathrm{II}}+\frac{1}{2} w_{\mathrm{III}}\right) \\
+\tau_{p l} h^{2}\left(\begin{array}{c}
\frac{1}{2} \frac{w_{\mathrm{I}}\left(l+w_{\mathrm{I}} \tan \alpha\right)}{\sqrt{\left(l w_{\mathrm{I}}\right)^{2}+h^{2}\left(l+w_{\mathrm{I}} \tan \alpha\right)^{2}}} \\
+\frac{w_{\mathrm{II}} \tan ^{2}}{\sqrt{l^{2}+h^{2} \tan ^{2} \alpha}} \\
-\frac{1}{2} \frac{w_{\mathrm{III}}\left(l-w_{\mathrm{III}} \tan \alpha\right)}{\sqrt{\left(l w_{\mathrm{III}}\right)^{2}+h^{2}\left(l-w_{\mathrm{III}} \tan \alpha\right)^{2}}}
\end{array}\right)
\end{gathered}
$$




\section{Appendix B: Geometry and material aspects of adhesion}

To determine adhesion energies, the non-retarded Van der Waals interaction free energy between two bodies, an atomic approach is used that is extended to bodies by pairwise additivity. The equation that is used for the energy between two atoms is [7]:

$$
W_{a t-a t}=-\frac{C_{a b}}{d^{6}}
$$

Equation (B.1) gives the interaction energy between the atoms $a$ and $b$ with a constant $C_{a b}$ that gives the interaction at a unit distance, dependent on the types of atoms $a$ and $b$ and $d$ the distance between the atoms. The interaction energy between an atom of type $a$ and a body consisting of atom type $b$, simply given as body $b$, can be calculated, using the density of atoms in body $b, \rho_{b}$ :

$$
W_{b o d y-a t}=-C_{a b} \iiint \frac{\rho_{b}}{d^{6}} d V
$$

Given a body that is defined as a half space with its boundary plane at a distance of $D$ from the atom, as given in figure B.1 gives:

$$
W_{b o d y-a t}=-C_{a b} \rho_{b} \int_{0}^{\infty} \int_{0}^{\infty} \frac{2 \pi r d r}{\left(r^{2}+(D+z)^{2}\right)^{3}} d z=-\frac{\pi C_{a b} \rho_{b}}{2} \int_{0}^{\infty} \frac{d z}{(D+z)^{4}}=-\frac{\pi C_{a b} \rho_{b}}{6 D^{3}}
$$

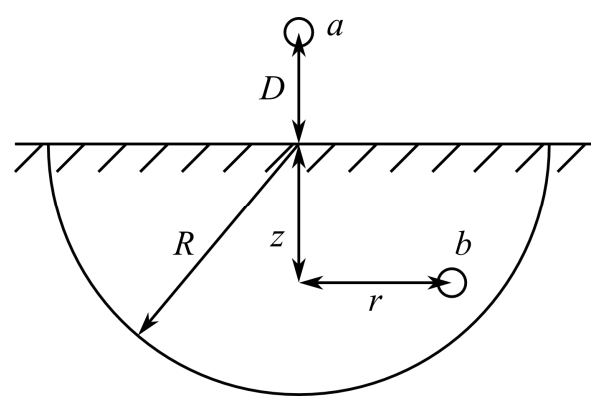

Figure B.1. Dimensions in interaction energy calculation.

If the interaction energy of equation (B.3) not only acts on a single atom, but also on a whole body made of atom type $a$, called body $a$, the result of equation (B.3) should be integrated over the whole body $a$. Taking for this body again a half space, separated with a distance $D$ from the other half space, the interaction energy per unit area becomes: 


$$
W_{b o d y-b o d y}=-\frac{\pi C_{a b} \rho_{a} \rho_{b}}{6} \int_{0}^{\infty} \frac{d z}{(D+z)^{3}}=-\frac{\pi C_{a b} \rho_{a} \rho_{b}}{12 D^{2}}=-\frac{A_{H}}{12 \pi D^{2}}
$$

The last term of equation (B.4) contains $A_{H}$, the Hamaker constant, which is discussed in section 4.4. The result of equation (B.4) is a result that can be found in adhesion literature, like [26].

The question arises how far the interacting bodies can be downscaled, so that equation (B.4) still gives a reasonable answer. Equation (B.3) will be derived again, where the half space body $b$ is replaced by half a ball with radius $R$ as shown in figure B.1. Atom $a$ is located at the symmetry axis of body $b$.

$$
\begin{aligned}
& W_{\text {ball-at }}=-C_{a b} \rho_{b} \int_{z=0}^{R} \int_{r=0}^{\sqrt{R^{2}-z^{2}}} \frac{2 \pi r d r}{\left(r^{2}+(D+z)^{2}\right)^{3}} d z \\
& =-\frac{\pi C_{a b} \rho_{b}}{2} \int_{z=0}^{R}\left(\frac{1}{(D+z)^{4}}-\frac{1}{\left(R^{2}+D^{2}+2 D z\right)^{2}}\right) d z \\
& =-\frac{\pi C_{a b} \rho_{b}}{2}\left(\frac{1}{2 D\left(D^{2}+2 D R+R^{2}\right)}-\frac{1}{3(D+R)^{3}}-\frac{1}{2 D\left(D^{2}+R^{2}\right)}+\frac{1}{3 D^{3}}\right)
\end{aligned}
$$

The result of equation (B.5) shows that as $R$ is an order of magnitude larger than $D$, the terms that contain $R$ in the denominator are two or three orders of magnitude smaller than the last term. This last term equals the answer in the case of a half space. So, as long as $R>>D$, the model assuming a half space is justified.

The next step is the determination of the interaction energy of equation (B.5) that acts on a body $a$ instead of an atom. The interaction energy between body $a$ and $b$ will be calculated per unit area of $a$ at the symmetry axis of $b$. Assumed is that body $a$ is a plate with a thickness $R$ and is located at a distance $D$ from $b$. This results in the following:

$$
\begin{aligned}
& W_{\text {ball-body }}=-\frac{\pi C_{a b} \rho_{a} \rho_{b}}{2} \int_{D}^{D+R}\left(\frac{1}{2 z\left(z^{2}+2 z R+R^{2}\right)}-\frac{1}{3(z+R)^{3}}-\frac{1}{2 z\left(z^{2}+R^{2}\right)}+\frac{1}{3 z^{3}}\right) d z \\
& =-\frac{A_{H}}{12 \pi D^{2}}\left(\frac{3}{\bar{R}^{2}} \ln \left(\frac{1+\bar{R}}{1+2 \bar{R}}\right)+\frac{3}{2 \bar{R}^{2}} \ln \left(\frac{(1+\bar{R})^{2}+\bar{R}^{2}}{1+\bar{R}^{2}}\right)-\frac{13 \bar{R}^{2}+15 \bar{R}+4}{(1+\bar{R})^{2}(1+2 \bar{R})^{2}}+1\right)
\end{aligned}
$$

In equation (B.6) $\bar{R}$ is $R / D$. From equation (B.6) it follows that as $R$ is an order of magnitude larger than $D$, its effect decreases by two orders of magnitude. So, the result of equation (B.6) converged to the result given in equation (B.4) as is shown in figure B.2. In figure B.2 is shown $\Delta \bar{W}=\left(W_{\text {ball-body }}-W_{\text {body-body }}\right) / W_{\text {body-body }}$ as defined by equations (B.4) and (B.6) as a function of $\bar{R}$. From this graph it follows that the relative error of equations 
(B.4) is reduced already to $4 \%$ in the case where $R$ is $10 D$. Assuming that $D$ equals the atomic distance, equation (B.4) can already be used for very small particles.

It can be concluded that the real geometry has small effect on the interaction energy. This means that only considering the geometry independent part is sufficient.

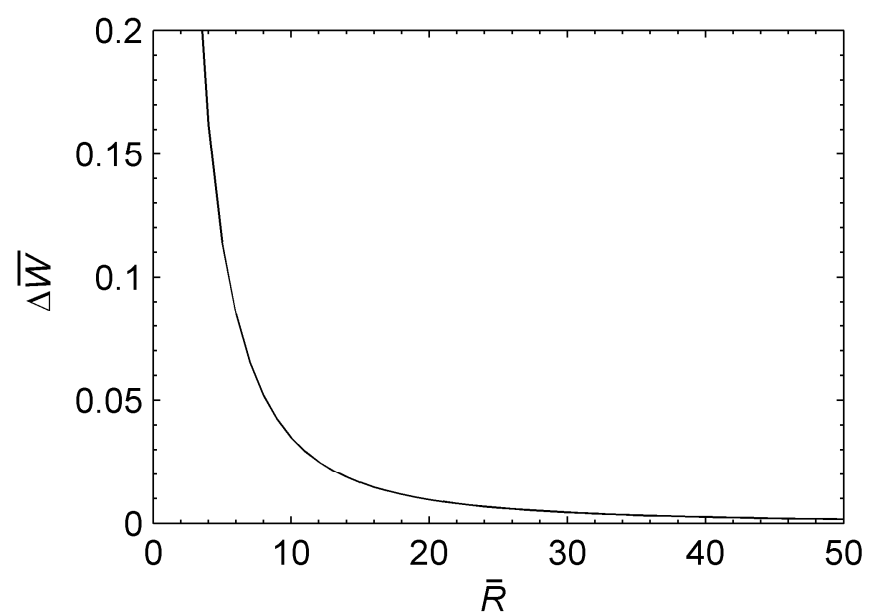

Figure B.2. Normalized difference on interaction energy between a half space and half a normalized ball with radius $\bar{R}$.

The Hamaker constant, as given above, is based on the assumption that pairwise additivity can be used. However, the Van der Waals force between two atoms will change due to the presence of a third atom. This means that the assumption of pairwise additivity of molecular interactions is not valid in general. This has consequences for the value of $A_{H}$, but the interaction energy remains proportional to $1 / d^{6}$, so the geometrical relations remain valid.

Pairwise additivity gives an $A_{H}$ as a function of $C_{a b}, \rho_{a}$ and $\rho_{b}$. As mentioned above, in general it cannot be stated that the derived $A_{H}$ has a proper value. Other theories exist, which avoid the shortcomings of additivity, like the Lifshitz theory [7]. This theory derives the forces between bodies on the basis of bulk properties as the dielectric constants and the refractive indices, instead of atomic structure. The bodies are treated as continuous media. Only the value of $A_{H}$ is calculated in a different manner, but the geometrical relations remain valid. This theory will not be worked out here. 


\section{Bibliography}

[1] A.G. Atkins and J.H. Liu, Toughness and the transition between cutting and rubbing in abrasive contacts, Wear, Vol. 262, pp. 146 - 159, 2007

[2] B. Avitzur, C.K. Huang and Y.D. Zhu, A friction model based on the upper-bound approach to the ridge and sublayer deformations, Wear, Vol. 95, pp. 59 - 77, 1984

[3] A.H. van den Boogaard, Thermally enhanced forming of aluminium sheet, $\mathrm{PhD}$ thesis, University of Twente, 2002

[4] J. Bos, Frictional heating of tribological contacts, $\mathrm{PhD}$ thesis, University of Twente, 1995

[5] J. Bos and H. Moes, Frictional heating of tribological contacts, Journal of Tribology, Vol. 117, pp. 171 - 177, 1995

[6] T. Burakowski and T. Wierzchon, Surface Engineering of Metals, CRC Press, 1999

[7] H.J. Butt, K. Graf and M. Kappl, Physics and Chemistry of Interfaces, Wiley-VCH, 2003

[8] B.D. Carleer, Finite element analysis of deep drawing, $\mathrm{PhD}$ thesis, University of Twente, 1997

[9] J. Chakrabarty, Theory of Plasticity, Elsevier, 2006

[10] J.M. Challen and P.L.B. Oxley, An explanation of the different regimes of friction and wear using asperity deformation models, Wear, Vol. 53, pp. 229 - 243, 1979

[11] J.M. Challen, P.L.B. Oxley and E.D. Doyle, The effect of strain hardening on the critical angle for abrasive (chip formation) wear, Wear, Vol. 88, pp. 1 - 12, 1983

[12] B.B. Chaudhuri and G.P. Samanta, Elliptic fit of objects in two and three dimensions by moment of inertia optimization, Pattern Recognition Letters, Vol. 12, pp. 1 - 7 , 1991

[13] H. Czichos, Tribology: A systems approach to the science and technology of friction, lubrication and wear, Tribology Series, 1, Elsevier Scientific Publishing Company, Amsterdam, Oxford, New York, 1978

[14] W.C. Emmens, Tribology of flat contacts and its application in deep drawing, $\mathrm{PhD}$ thesis, University of Twente, 1997

[15] A. Gåård, P. Krakhmalev and J. Bergström, Wear mechanisms in deep drawing of carbon steel - correlation to laboratory testing, Tribotest, Vol. 14, pp. 1 - 9, 2008

[16] J.A. Greenwood, A Unified Theory of Surface Roughness, Proceedings of the Royal Society of London. Series A, Mathematical and Physical Sciences, Vol. 393, pp. $133-157,1984$

[17] M. Hanson, On adhesion and galling in metal forming, $\mathrm{PhD}$ thesis, Uppsala University, 2008

[18] W.M. Haynes, Handbook of Chemistry and Physics, CRC, 2010 
[19] E. van der Heide, A.J. Huis in 't Veld and D.J. Schipper, The effect of lubricant selection on galling in a model wear test, Wear, Vol. 251, pp. 973 - 979, 2001

[20] E. van der Heide, Lubricant failure in sheet metal forming processes, $\mathrm{PhD}$ thesis, University of Twente, 2002

[21] E. van der Heide and D.J. Schipper, Galling initiation due to frictional heating, Wear, Vol. 254, pp. 1127 - 1133, 2003

[22] E. van der Heide and D.J. Schipper, On the Frictional Heating in Single Summit Contacts: Towards Failure at Asperity Level in Lubricated Systems, Journal of Tribology, Vol. 126, pp. 275 - 280, 2004

[23] V.E. Henrich, The surface science of metal oxides, Cambridge University Press, 1994

[24] K. Hokkirigawa and K. Kato, An experimental and theoretical investigation of ploughing, cutting and wedge formation during abrasive wear, Tribology International, Vol. 21, pp. 51 - 57, 1988

[25] M.-K. Hu, Visual pattern recognition by moment invariants, Information Theory, IRE Transactions on, Vol. 8, pp. 179 - 187, 1962

[26] J. Israelachvili, Intermolecular \& Surface Forces, Academic Press, 1991

[27] K.L. Johnson, Adhesion at the contact of solids, In W.T. Koiter, editor, Theoretical and Applied Mechanics, pp. 133 - 143, North-Holland Publishing Company, 1976

[28] D.A. Korzekwa, P.R. Dawson and W.R.D. Wilson, Surface asperity deformation during sheet forming, International Journal of Mechanical Sciences, Vol. 34, pp. 521 - 539, 1992

[29] S. Lafaye, C. Gauthier and R. Schirrer, A surface flow line model of a scratching tip: apparent and true local friction coefficients, Tribology International, Vol. 38, pp. $113-127,2005$

[30] Y.W. Lee and H.I. Aaronson, Surface concentration profile and surface energy in binary alloys, Surface Science, Vol. 95, pp. 227 - 244, 1980

[31] Y. Li and D.Y. Li, Experimental studies on relationships between the electron work function, adhesion, and friction for $3 \mathrm{~d}$ transition metals, Journal of Applied Physics, Vol. 95, pp. 7961 - 7965, 2004

[32] Y. Li and D.Y. Li, Electron work function, adhesion, and friction between 3d transition metals under light loads, Wear, Vol. 259, pp. 1432 - 1436, 2005

[33] Y. Li and D.Y. Li, An energy consumption model based on the electron work function and Fermi energy for predicting adhesion and low-load friction between $3 \mathrm{~d}$ transition metals, Wear, Vol. 263, pp. 1610 - 1615, 2007

[34] F.W. Lindvall, Development of a test method for measuring galling resistance, Master thesis, Karlstad University, 2007

[35] M.A. Masen, Abrasive tool wear in metal forming processes, $\mathrm{PhD}$ thesis, University of Twente, 2004 
[36] M. A. Masen and M. B. de Rooij, Abrasive wear between rough surfaces in deep drawing, Wear, Vol. 256, pp. 639 - 646, 2004

[37] J. Pullen and J.B.P. Williamson, On the Plastic Contact of Rough Surfaces, Proceedings of the Royal Society of London. Series A, Mathematical and Physical Sciences, Vol. 327, pp. 159 - 173, 1972

[38] E. Rabinowicz, Friction and wear of materials, John Wiley \& Sons, 1995

[39] M.B. de Rooij, Tribological aspects of unlubricated deepdrawing processes, $\mathrm{PhD}$ thesis, University of Twente, 1998

[40] M.B. de Rooij and D.J. Schipper, Analysis of Material Transfer From a Soft Workpiece to a Hard Tool: Part I - Lump Growth Model, Journal of Tribology, Vol. 123, pp. 469 - 473, 2001

[41] E. Schedin and B. Lehtinen, Galling mechanisms in lubricated systems: A study of sheet metal forming, Wear, Vol. 170, pp. 119 - 130, 1993

[42] E. Schedin, Galling mechanisms in sheet forming operations, Wear, Vol. 179, pp. $123-128,1994$

[43] A.K. Sengupta, B. Fogg and S.K. Ghosh, On the mechanism behind the punch-blank surface conformation in stretch-forming and deep-drawing, Journal of Mechanical Working Technology, Vol. 5, pp. 181 - 210, 1981

[44] R.J.J.M. Sniekers, Friction in deep drawing, $\mathrm{PhD}$ thesis, University of Technology Eindhoven, 1996

[45] M. de Vathaire, F. Delamare and E. Felder, An upper bound model of ploughing by a pyramidal indenter, Wear, Vol. 66, pp. 55 - 64, 1981

[46] L. Vitos, A.V. Ruban, H.L. Skriver and J. Kollár, The surface energy of metals, Surface Science, Vol. 411, pp. 186 - 202, 1998

[47] J.D. Westeneng, Modelling of contact and friction in deep drawing processes, $\mathrm{PhD}$ thesis, University of Twente, 2001

[48] W.R.D. Wilson and S. Sheu, Real area of contact and boundary friction in metal forming, International Journal of Mechanical Sciences, Vol. 30, pp. 475 - 489, 1988 